NIST Technical Note 2000

\title{
Database-Assisted Design and Equivalent Static Wind Loads for Mid- and High-Rise Structures: Concepts, Software, and User's Manual
}

Sejun Park

DongHun Yeo

This publication is available free of charge from: https://doi.org/10.6028/NIST.TN.2000

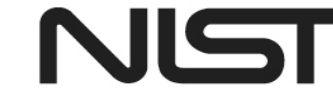

National Institute of Standards and Technology U.S. Department of Commerce 


\section{NIST Technical Note 2000}

\section{Database-Assisted Design and Equivalent Static Wind Loads for Mid- and High-Rise Structures: Concepts, Software, and User's Manual}

Sejun Park

DongHun Yeo

Engineering Laboratory National Institute of Standards and Technology

Gaithersburg, MD 20899-7311

This publication is available free of charge from:

https://doi.org/10.6028/NIST.TN.2000

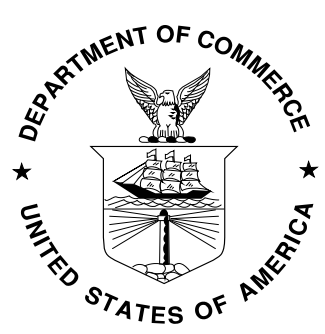

U.S. Department of Commerce Wilbur L. Ross, Jr., Secretary

National Institute of Standards and Technology Walter Copan, NIST Director and Undersecretary of Commerce for Standards and Technology 
Certain commercial entities, equipment, or materials may be identified in this document in order to describe an experimental procedure or concept adequately. Such identification is not intended to imply recommendation or endorsement by the National Institute of Standards and Technology, nor is it intended to imply that the entities, materials, or equipment are necessarily the best available for the purpose.

National Institute of Standards and Technology Technical Note 2000

Natl. Inst. Stand. Technol. Tech. Note 2000, 125 pages (June 2018)

CODEN: NTNOEF

This publication is available free of charge from:

https://doi.org/10.6028/NIST.TN.2000 


\section{Abstract}

This report presents the software DAD_ESWL v1.0, capable of computing efficiently the response to wind of mid- and high-rise structures consisting of up to thousands of members. The report documents the following features of the software:

(1) Use of the Database-Assisted Design (DAD) or of the Equivalent Static Wind Loads (ESWL) procedure for the iterative structural design of mid- and high-rise buildings.

(2) Incorporation into the design of second-order elastic analysis (both $P-\triangle-$ member chord rotation - and $P-\delta$ - member curvature). For purposes of illustration, DAD_ESWL uses a geometric stiffness method allowing second-order effects to be accounted for with no iterations.

(3) A graphical user interface, including: (i) definition of pressure tap locations, (ii) pressure distribution displays, (iii) calculation of floor wind loads induced by simultaneous time series of pressures at the taps, and (iv) use of MATLAB tools enabling convenient editing of computation result displays.

(4) Updates of steel member interaction formulas applicable to various types of beamcolumn joints, bracing connection details, and cross-sectional shapes.

(5) Generation of biaxial interaction strength surfaces for determining the demand-tocapacity indexes of reinforced concrete columns or shear walls with rectangular cross section specified by the user. The demand-to-capacity index for the shear wall can be calculated as well.

(6) Inclusion of the capability to use OpenSees for the efficient calculation of modal shapes and frequencies of vibration, as well as of influence coefficients.

The report includes a detailed user's manual. 


\section{Acknowledgements}

The wind tunnel data were developed at the CRIACIV-DIC Boundary Layer Wind Tunnel and were kindly provided by Dr. Ilaria Venanzi of the University of Perugia. Dr. Joseph A. Main provided the material on the time integration of modal equations via ramp-invariant filters. The thorough review, especially in demand-to-capacity indexes for reinforced concrete members by Prof. Chungwook Sim of University of Nebraska-Lincoln is acknowledged with thanks. 


\section{Contents}

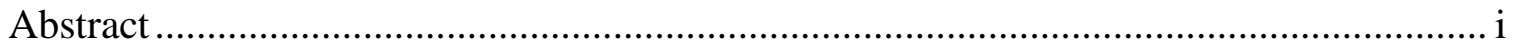

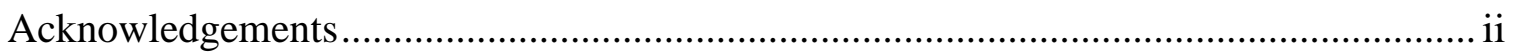

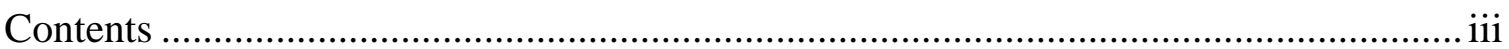

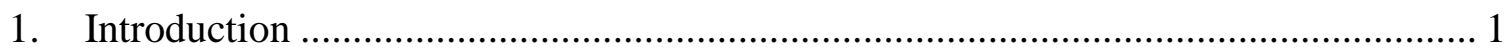

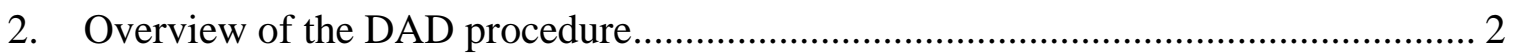

3. Overview of the Equivalent Static Wind Loads (ESWL) Procedure ......................... 5

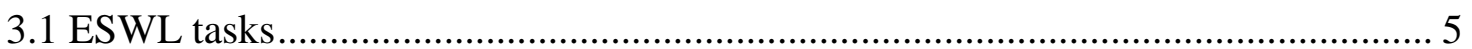

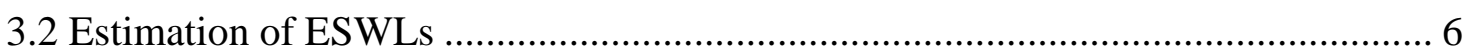

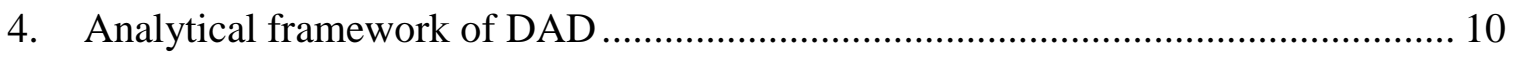

4.1 Dynamic modeling of tall building including second-order effect....................... 10

4.2 Aerodynamic database and wind load modeling .............................................. 12

4.3 Influence coefficients for determining internal forces ...................................... 14

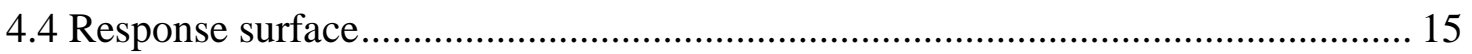

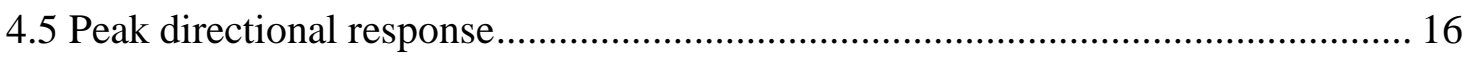

4.6 Peak wind effects with specified MRIs in regular and mixed wind climates ......... 17

4.7 Adjustment of demand-to-capacity indexes..................................................... 18

4.8 Compliance with design criteria .................................................................... 19

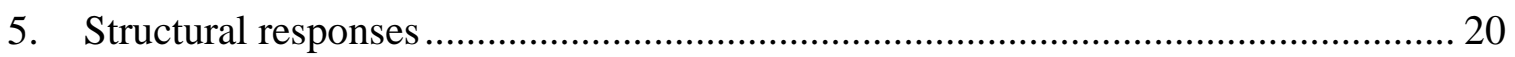

5.1 Demand-to-capacity indexes for steel members ............................................ 20

5.2 Demand-to-capacity indexes for RC members ................................................. 21

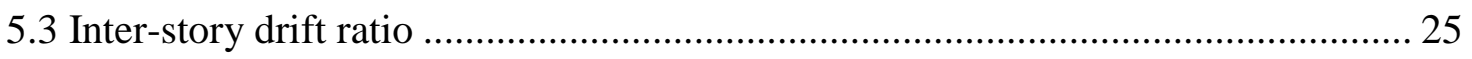

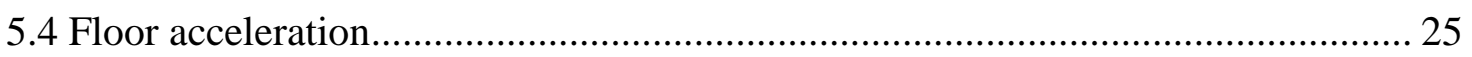

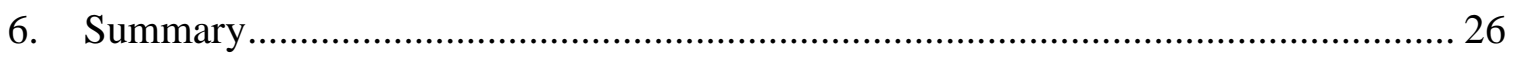

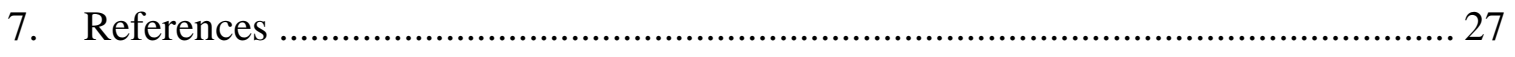

Appendix I. User’s Manual for DAD_ESWL v1.0................................................. A1

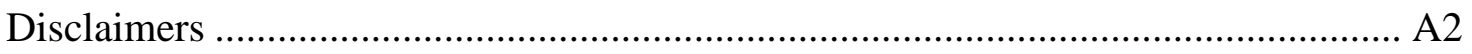

A1.1. Key features of DAD_ESWL software.................................................... A3

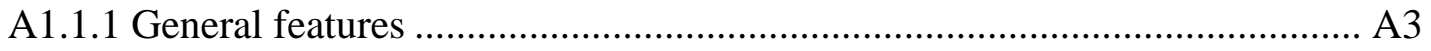

A1.1.2 Special features for steel structures ................................................... A4

A1.1.3 Special features for RC structures.................................................... A4

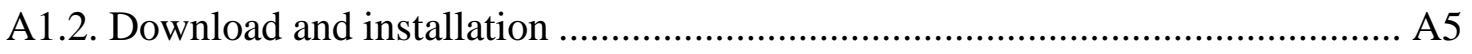

A1.3. Basics of using the DAD_ESWL ............................................................ A5 


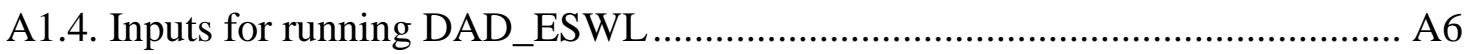

A1.5. Outputs from running DAD_ESWL ……………....................................... A7

A1.6. Details on use of the software ................................................................... A7

A1.6.1 Example of a 3D 60-story steel building …………................................ A7

A1.6.2 Example of a 3D 60-story reinforced concrete building.............................. A8

A1.6.3 Graphical user interface .................................................................... A9

Appendix II. Time integration of modal equations via ramp-invariant filters............... A54

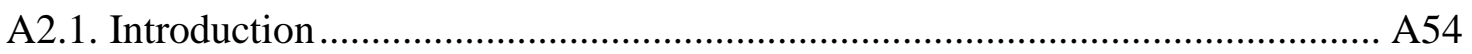

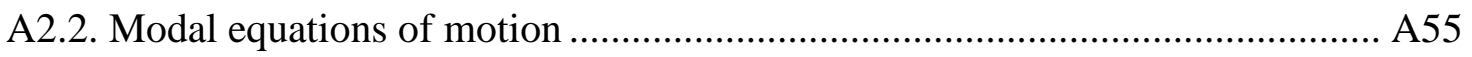

A2.3. Ramp-invariant filter for modal displacements .............................................. A55

A2.4. Ramp-invariant filter for modal velocities..................................................... A58

A2.5. Verification of the solver .......................................................................... A60

A2.5.1 Single-degree-of-freedom system .......................................................... A60

A2.5.1.1 Closed-form solution.................................................................... A60

A2.5.1.2 Numerical solution ...................................................................... A61

A2.5.1.3 Comparison between closed-form and numerical solutions ................ A62

A2.5.2 Multi-degree-of-freedom system ............................................................ A63

A2.5.2.1 Closed-form solution........................................................................ A63

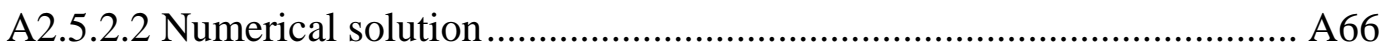

A2.5.2.3 Comparison between closed-form and numerical solutions ............... A67

Appendix III. Verification of bi-axial interaction surfaces............................................. A68

A3.1. Example section 1................................................................................... A69

A3.1. Example section 2 2.................................................................................. A70

A3.1. Example section 3 ……........................................................................ A71

Appendix IV. Example Tcl script for running OpenSees .............................................. A72

Appendix V. Construction of variable - 'mass' ........................................................... A77

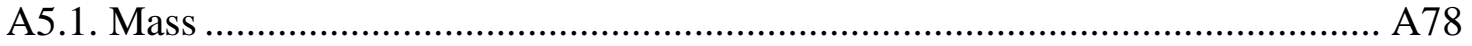

A5.2. Mass moment of inertia............................................................................. A79

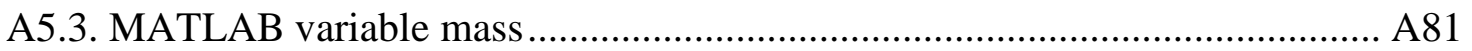

Appendix VI. Construction of variable - 'dif' ............................................................ A82

Appendix VII. Construction of variable - 'Ratio_Vs'................................................... A85

A7.1. Wind speed profiles and micro-meteorological homogeneity ......................... A85

A7.2. Example problems...................................................................................... A89 


\section{Introduction}

The ASCE 7-16 Standard (hereinafter ASCE 7-16) (ASCE 2016) specifies three methods for determining wind loads: the simplified, the analytical, and the wind tunnel method. The simplified method is not applicable to flexible, high-rise structures. The analytical method excludes buildings subjected to across-wind and/or aeroelastic effects. Since most mid-rise and tall buildings experience such effects, the analytical procedure is usable only for preliminary design purposes. The wind tunnel method is specified in ASCE 7-16 only in very general terms. This is one of the reasons why estimates of wind effects for the same structure may vary, in some cases significantly, among independent laboratories or even within the same laboratory (Coffman et al. 2010; Fritz et al. 2008; SOM 2004).

The increasingly common use of pressure scanners has led to the development of procedures for estimating aerodynamic wind loads by using (i) simultaneously measured time series of pressure coefficients at large numbers of pressure taps on wind tunnel building models, and (ii) measured or simulated extreme directional wind speeds at the building site (Boggs and Lepage 2006; Garber et al. 2007; Ho et al. 2014; Huang and Chen 2007). For the determination of design static wind loads it is typically assumed that the most unfavorable combination of wind loads will occur when the resultant wind loads along one of the principal axes of the building, or the wind-induced torsional moment, attains its peak value. Considering the combinations of translational and torsional loads, 24 load combinations are typically defined for design purposes (Ho et al. 2014). The static wind loads and the randomly fluctuating wind loads based on the entire time series of the measured pressure coefficients are called equivalent if they induce in the structural members' demand-to-capacity indexes (DCIs) with approximately the same values. However, depending upon the method of estimation of the static loads, the latter may not be equivalent to their randomly fluctuating counterparts, in which case the static wind loads would result in at least some structural members being under- or over-designed.

The purpose of this report is to review the Database-Assisted Design (DAD) approach, and to present and assess the equivalent static wind load (ESWL)approach, by which the randomly fluctuating wind loads used in the DAD procedure are replaced by ESWL.

The DAD and ESWL approaches allow a clear separation of the wind engineer's and structural engineer's tasks. The wind engineer's task is to provide: (1) the requisite pressure time histories based on wind tunnel testing or, as is likely to be the case in the future, on CWE (Computational Wind Engineering) simulations; (2) wind climatological directional data at a weather station reasonably representative of the wind climate at the building site and/or developed by, e.g., Monte Carlo simulations; (3) the ratio between directional wind speeds at the weather station and the reference directional mean wind speeds at the top of the building as affected by the building's exposure; and (4) measure of the uncertainties in the aerodynamic, wind climatological, and micrometeorological data. Once these aerodynamic, wind climatological, and micrometeorological data are provided by the wind engineer, the structural engineer is in full charge of the design and uses those data in, typically, an iterative design process that accounts rigorously for wind directionality effects. That process results in a safe, economical and risk-consistent design that fully satisfies strength and serviceability performance criteria. Sections 2 and 3 present an overview of the DAD procedure and of the ESWL procedure, respectively. 
All the operations described in this report are automated, and are performed by using the DAD_ESWL (Database-Assisted Design and Equivalent Static Wind Load for midand high-rise structures) software. A detailed user's manual is included in Appendix I.

\section{Overview of the DAD procedure}

The DAD procedure as applied to mid- and high-rise buildings entails the tasks represented in Fig. 1. The processes within the dotted box constitute the main algorithm of the DAD_ESWL software. To use the DAD procedure the user selects the DAD option of the software. The processes outside the box describe information provided by the wind engineer and the structural engineer. Their tasks are clearly separated as follows. The yellow (bright) blocks and the blue (dark) blocks correspond to the wind engineer's and the structural engineer's tasks, respectively. Uncertainty estimates are beyond the scope of this report. For the DAD procedure the structural engineer performs the following tasks:

1) Select the structural system, and determine its preliminary member sizes based on a simplified model of the wind loading (e.g., a static wind loading based on standard provisions). The structural design so achieved is denoted by $D_{0}$.

2) For the design $D_{0}$ : determine the system's mechanical properties, including the modal shapes, natural frequencies of vibration, and damping ratios, as well as the requisite influence coefficients; and develop a lumped-mass model of the structure. $P-\Delta$ an $P-\delta$ effects are accounted in this report by using a geometric stiffness matrix approach (Park and Yeo 2018). This approach has limitations noted in Park and Yeo (2018) that appear not to be significant for tall buildings subjected to wind loads. It is suggested, however, that the validity of this statement be the object of further research, and that an alternative approach to the estimation of second-order effects be considered if necessary.

3) From the time histories of simultaneously measured pressure coefficients, determine the time histories of the randomly varying aerodynamic loads induced at all floor levels by mean wind speeds from, depending upon location, $10 \mathrm{~m} / \mathrm{s}$ to $80 \mathrm{~m} / \mathrm{s}$ in increments of 10 $\mathrm{m} / \mathrm{s}$, with directions from $0^{\circ} \leq \theta_{w}<360^{\circ}$ typically in increments of, say, $10^{\circ}$ (the reference height for the mean wind speeds is typically assumed to be the height of the structure).

Tasks 4, 5, and 6 are performed for each of those directional wind speeds and are required for the determining, by accounting rigorously for directionality, the requisite wind effects with the specified design mean recurrence interval (MRI).

4) Perform the dynamic analysis based on the lumped-mass model of the structure to obtain the time histories of the inertial forces induced by the respective aerodynamic loads,

and the effective wind-induced loads consisting of the sums of the aerodynamic and inertial force time histories.

5) For each cross section of interest, use the appropriate influence coefficients to determine time series of the demand-to-capacity indexes (DCIs) induced by the combination of effective wind loads determined in task 4 with factored gravity loads. The 
DCIs are the left-hand sides of the design interaction equations, and are typically used to size members subjected to more than one type of internal force. For example, the interaction equations for steel members subjected to flexure and axial forces are (ANSI/AISC 2010):

$$
\begin{array}{lll}
\text { When } \frac{P_{r}}{\phi_{p} P_{n}} \geq 0.2, & \frac{P_{r}}{\phi_{p} P_{n}}+\frac{8}{9}\left(\frac{M_{r x}}{\phi_{m} M_{n x}}+\frac{M_{r y}}{\phi_{m} M_{n y}}\right) \leq 1.0 \\
\text { When } \frac{P_{r}}{\phi_{p} P_{n}}<0.2, & \frac{P_{r}}{2 \phi_{p} P_{n}}+\left(\frac{M_{r x}}{\phi_{m} M_{n x}}+\frac{M_{r y}}{\phi_{m} M_{n y}}\right) \leq 1.0
\end{array}
$$

In Eqs. 1a and 1b, $P_{r}$ and $P_{n}$ are the required and available tensile or compressive strength; $M_{r x}$ and $M_{n x}$ are the required and available flexural strength about the strong axis; $M_{r y}$ and $M_{n y}$ are the required and available flexural strength about the weak axis; $\phi_{p}$ and $\phi_{m}$ are resistance factors. The required strengths are based on combinations of wind and gravity effects specified in Section 2.2 of ASCE 7-16 (ASCE 2016) or other standards. A similar, though simpler expression for the DCI, is applied to shear forces.

6) Construct the response surfaces of the peak combined effects (e.g., DCIs, inter-story drift ratio, accelerations) as functions of wind speed and direction. For each of the directional wind speeds defined in task 3, determine for each cross section of interest the peak of the DCI time series (e.g., Eqs. 1a and 1b), and construct from the results so obtained a peak DCI response surface. The response surface is a property of the aerodynamic and mechanical characteristics of the structure, independent of the wind climate, that provides for each cross section of interest the peak DCIs (or other wind effects) as a function of wind speed and direction.

7) Use the information contained in the response surfaces and in the matrices of directional wind speeds at the site to determine, by accounting for wind directionality, the design DCIs with the specified design MRI $\bar{N}$, denoted by $D C I^{\text {design }}(\bar{N})$, for the cross sections of interest. The steps required for this purpose are described in detail in the Section 4.6.

Typically, the preliminary design $D_{0}$ does not satisfy the strength and/or serviceability design criteria. The structural members are then re-sized to produce a modified structural design $D_{1}$. This iterative process continues until the final design is satisfactory. Tasks 2 through 7 are repeated as necessary until the design DCIs are close to unity, to within serviceability constraints. Each iteration entails a re-sizing of the structural members consistent with the respective estimated design DCIs. 


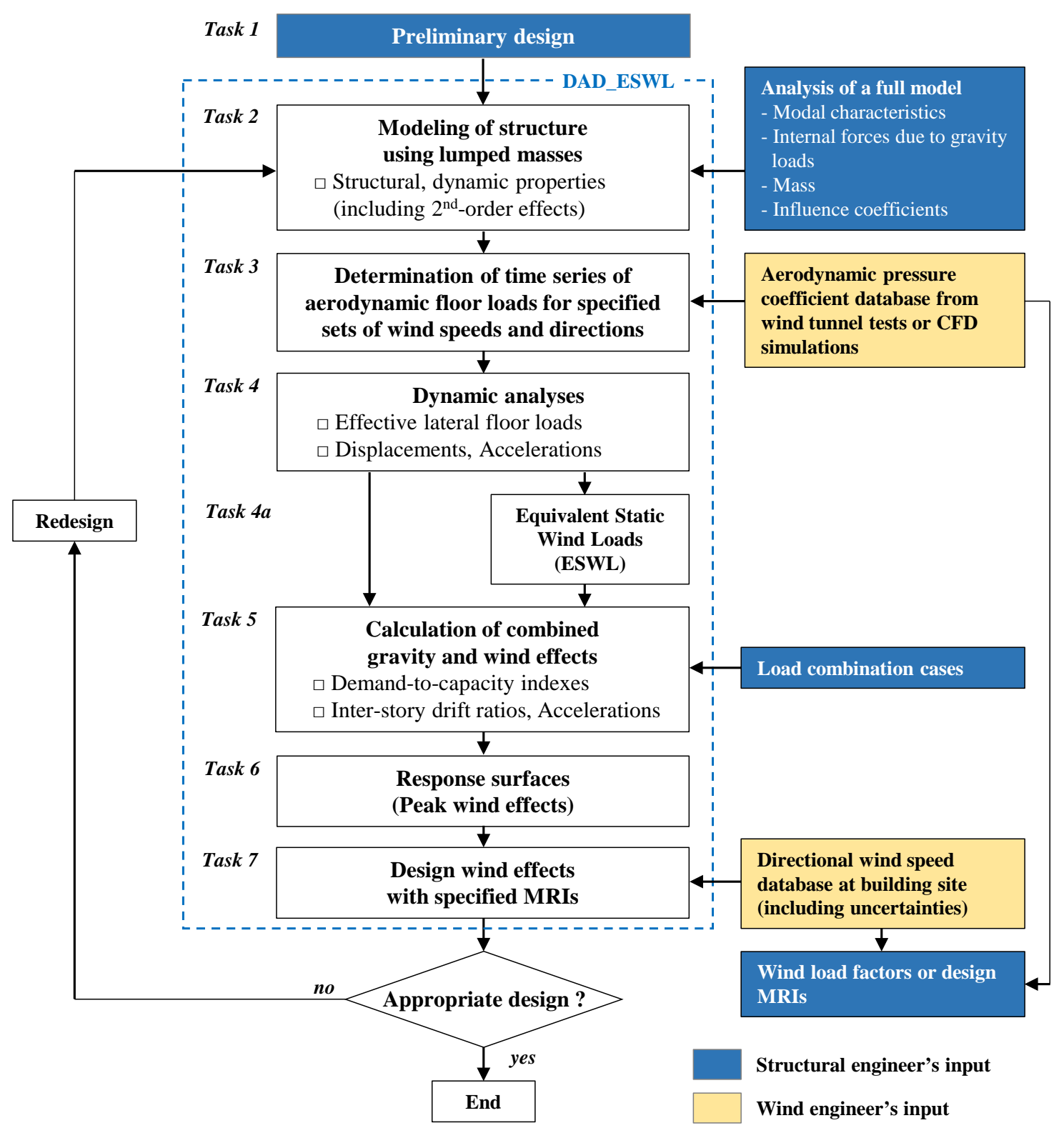

Figure 1. DAD_ESWL procedure 


\section{Overview of the Equivalent Static Wind Loads (ESWL) Procedure}

\subsection{ESWL tasks}

Like DAD, the ESWL procedure requires the wind engineer to provide (i) wind climatological data at the building site, (ii) time series of pressure coefficients measured simultaneously at multiple taps, and (iii) uncertainty estimates for both the wind climatological and the aerodynamic data. Once these three tasks are completed, the design process is fully the responsibility of the structural engineer.

The first four tasks of the ESWL procedure are strictly identical to the first four tasks of the DAD procedure as listed in Section 2. The subsequent tasks are performed for each of the wind speeds and directions defined in task 3 (see Fig. 1).

4a) Determine the static loads $F_{k x, p}^{\mathrm{ESWL}}(U, \theta), F_{k y, p}^{\mathrm{ESWL}}(U, \theta)$, and $F_{k \vartheta, p}^{\mathrm{ESWL}}(U, \theta)$ acting at the center of mass of floor $k$ in the direction of the building's principal axes $x, y$ and in torsion, respectively, where the subscript $p\left(p=1,2, \ldots, p_{\max }\right)$ identifies distinct wind loading cases $\mathrm{WLC}_{p}$ associated with superpositions of ESWL load effects, and $p_{\max }$ is a function of the number $n_{p i t}$ of points in time used to obtain the peak effects of interest. This task is described in detail in Section 3.2.

5) For each cross section $m$ of interest, calculate the internal forces used to determine its DCI, and substitute their expressions into the expressions for the DCIs (e.g., Eq. 1). This task requires the use of the static wind loads determined in task 4a, the factored gravity loads, and the influence coefficients $r_{m k, x}, r_{m k, y}$, and $r_{m k, \vartheta}$. The influence coefficients represent internal forces at cross section $m$ induced by a unit floor load in the direction $x$, $y$, and $\vartheta$ applied at floor $k$, respectively. Denote the internal forces by $f_{m, p}^{\mathrm{ESWL}}(U, \theta)$. Their expression is

$$
f_{m, p}^{\mathrm{ESWL}}(U, \theta)=\sum_{k=1}^{n} r_{m k, x} F_{k x, p}^{\mathrm{ESWL}}(U, \theta)+\sum_{k=1}^{n} r_{m k, y} F_{k y, p}^{\mathrm{ESWL}}(U, \theta)+\sum_{k=1}^{n} r_{m k, \vartheta} F_{k \vartheta, p}^{\mathrm{ESWL}}(U, \theta)
$$

The corresponding demand-to-capacity indexes, denoted by $\operatorname{DCI}_{m, p}^{\mathrm{ESWL}}(U, \theta)$, are obtained by substituting internal forces determined by Eq. 2 into the expressions for the DCIs. For design purposes only the largest of these DCIs is of interest, that is,

$$
\operatorname{DCI}_{m}^{\mathrm{RS}, \mathrm{ESWL}}(U, \theta)=\max _{p}\left(\mathrm{DCI}_{m, p}^{\mathrm{ESWL}}(U, \theta)\right)
$$

6) Construct the response surfaces representing, for each cross section $m$ of interest, the dependence of its demand-to-capacity index $\operatorname{DCI}_{m}^{\mathrm{RS}, \mathrm{ESWL}}(U, \theta)$ upon wind speed $U$ and direction $\theta$.

7) Use the response surfaces constructed in task 6 and the non-parametric statistical procedure described in detail in Section 4.6 to determine, from the values $\operatorname{DCI}_{m}^{\mathrm{RS}, \mathrm{ESWL}}(U, \theta)$ 
, the design demand-to-capacity indexes with an $\bar{N}$-year mean recurrence interval, $\operatorname{DCI}_{m}^{\mathrm{pk}}(\bar{N})$. Depending upon the uncertainties in the wind velocity and aerodynamic data, as determined by the wind engineering laboratory, the design MRI may have to differ from the value specified in the ASCE 7-16 Standard (ASCE 2016).

If the DCIs determined in task 7 differ significantly from unity, the structure's members are re-sized to create a new design $D_{1}$. Tasks 2 to 7 are then performed on that design. This process is iterated until a structural design is achieved for which, in each structural member, the DCI induced by the most unfavorable combination of ESWLs is close to unity, to within serviceability constraints.

\subsection{Estimation of ESWLS}

This section describes a simple approach to structural design, wherein the peak DCIs produced by randomly fluctuating effective wind forces are replaced by their counterparts produced by equivalent static wind loads. For ease of exposition, the indexes $U$ and $\theta$ will be omitted in this section.

First the case of a structure subjected to wind loads acting only in one direction $x$ at its centers of mass. For any given wind speed, let the effective (i.e., aerodynamic plus inertia) randomly fluctuating load at floor $k$ acting along the principal axis $x$ of the building be denoted by $F_{k x}(t)$, where $k=1,2, \ldots, n$ (Fig. 2). The corresponding overturning moment at the base of the building is (Fig. 2a)

$$
M_{b y}(t)=h\left[F_{1 x}(t)+2 F_{2 x}(t)+\cdots+n F_{n x}(t)\right]
$$

where it is assumed for simplicity that all floors have the same height $h$. The peak of $M_{b y}(t)$, denoted by $\max _{t}\left[M_{b y}(t)\right]$, occurs at a time $t_{1 x}$ (Fig. 2a). The equivalent static wind load in direction $x$ at floor $k$, denoted by $F_{k x}^{\mathrm{ESWL}}$ is therefore

$$
F_{k x}^{\mathrm{ESWL}}=F_{k x}\left(t_{1 x}\right)
$$

and the moment at the structure's base is

$$
h\left(F_{1 x}^{\mathrm{ESWL}}+2 F_{2 x}^{\mathrm{ESWL}}+\cdots+n F_{n x}^{\mathrm{ESWL}}\right)=M_{b y}\left(t_{1 x}\right)
$$

The static wind loading determined as described above may be called equivalent static wind loading if it induces in all structural members DCIs approximately equal to their peak DAD counterparts.

The internal force at the member cross section $m$ induced by the effective fluctuating forces $F_{k x}(t)$ at floor $k(k=1,2, \ldots, n)$ can be written as

$$
f_{m}(t)=\sum_{k=1}^{n} r_{m k, x} F_{k x}(t)
$$


where $r_{m k, x}$ is the influence coefficients representing the internal forces at cross section $m$ induced by a unit load applied at floor $k$. The ESWLs $F_{k x}^{\mathrm{ESWL}}$ are determined as in Eq. 5. It is assumed that the peak internal forces occur at the same time $t_{x q}$ as the peak base moment. If this were true, the following system of equations would be satisfied:

$$
\max _{t}\left[f_{m}(t)\right]=\sum_{k=1}^{n} r_{m k, x} F_{k x}^{\mathrm{ESWL}} \quad\left(m=1,2, \ldots, m_{\max }\right)
$$

Eq. 8 would be rigorously true if the influence coefficients $r_{m k, x}$ were proportional to the height $k h$. However, it is shown in Park et al. (2018) that Eq. 8 (and, in particular, its counterpart wherein forces in the principal direction $y$ and torsional moments about the center of mass are also acting on the structure) can be satisfied to within a close approximation if wind directionality effects are accounted for. Note that this may not be the case if wind loads are dominant in one direction only.

For structures that experience wind-induced forces in the directions $x$ and $y$ of the structure's principal axes, as well as wind-induced torsional moments about the center of mass, wind effects are due to the superposition of the effects of these three actions. It is therefore possible to construct, from the time histories of those three effects, the time history of their summation, and estimate the peak of that time history. This approach can be used in the DAD procedure.

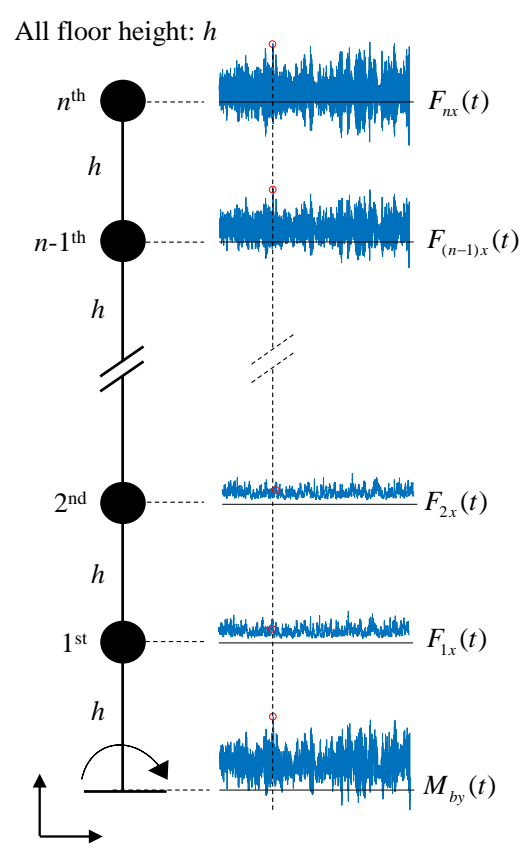

(a)

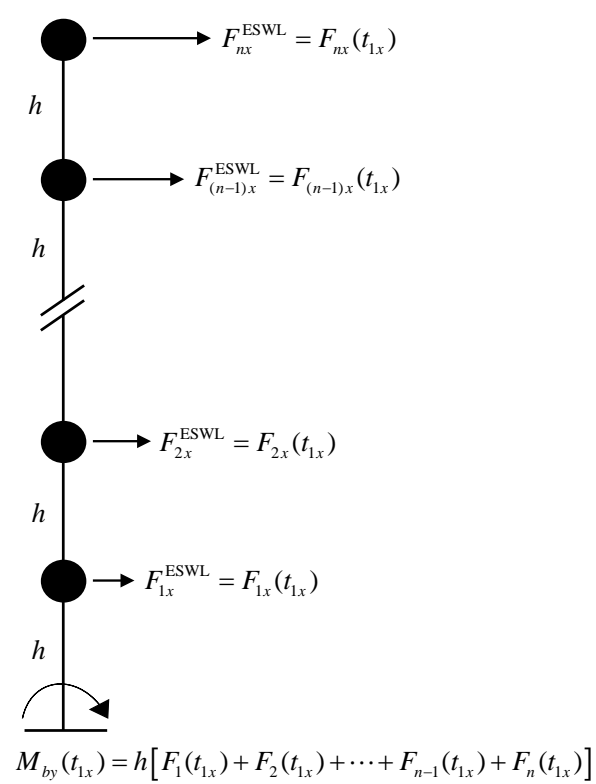

(b)

Figure 2. Lumped mass structure with (a) fluctuating wind loads in DAD and (b) equivalent static wind loads in ESWL. 
For the ESWL, a multiple points-in time (MPIT) (Yeo 2013) approach with a number $n_{\text {pit }}$ of points in time is used as follows (see Fig. 3). The highest peak of the time series of the base moments induced by forces in the $x$ direction, and its time of occurrence $t_{1 x}$, are identified. The forces $F_{k x}\left(t_{1 x}\right)$ are defined as the principal ESWLs at floors $k(k=1,2, \ldots$, $n)$ acting on the structure at time $t_{1 x}$. The forces $F_{k y}\left(t_{1 x}\right)$ and $F_{k g}\left(t_{1 x}\right)$ are defined as the companion ESWLs at floors $k$. Because the simultaneously acting forces $F_{k x}\left(t_{1 x}\right), F_{k y}\left(t_{1 x}\right)$ and $F_{k g}\left(t_{1 x}\right)$ do not necessarily produce the most unfavorable effect being considered, it is necessary to perform the operation just described for the second, ..., $n_{\text {pit }}$-th highest peak of the time series of the base moments induced by forces in the $x$ direction. Thus, a total of $n_{p i t}$ combinations of principal ESWLs and two companion ESWLs are obtained for times $t_{1 x}, t_{2 x}, \ldots, t_{n_{p i t} x}$.

Next, $n_{p i t}$ combinations of principal and companion ESWLs acting at times $t_{1 y}, t_{2 y}, \ldots$, $t_{n_{\text {pit }}}$ are obtained by considering the highest, second highest, ..., $n_{\text {pit }}$-th highest peak of the base moments induced by the forces in the direction $y$. A third set of $n_{\text {pit }}$ combinations acting at times $t_{19}, t_{29}, \ldots, t_{n_{\text {pit }} \vartheta^{9}}$ corresponds to the highest peaks of base moments induced by torsion. Three additional sets correspond to lowest peak base moments. Thus, $p_{\max }=$ $6 n_{\text {pit }}$ sets of equivalent static wind loading combinations must be used to determine the wind effects of interest. The design wind effect is the largest of the effects induced by these $6 n_{\text {pit }}$ sets of ESWLs.

The operations listed in this section are performed automatically using the ESWL option of the DAD_ESWL software. For details, see User's Manual of Appendix I. 

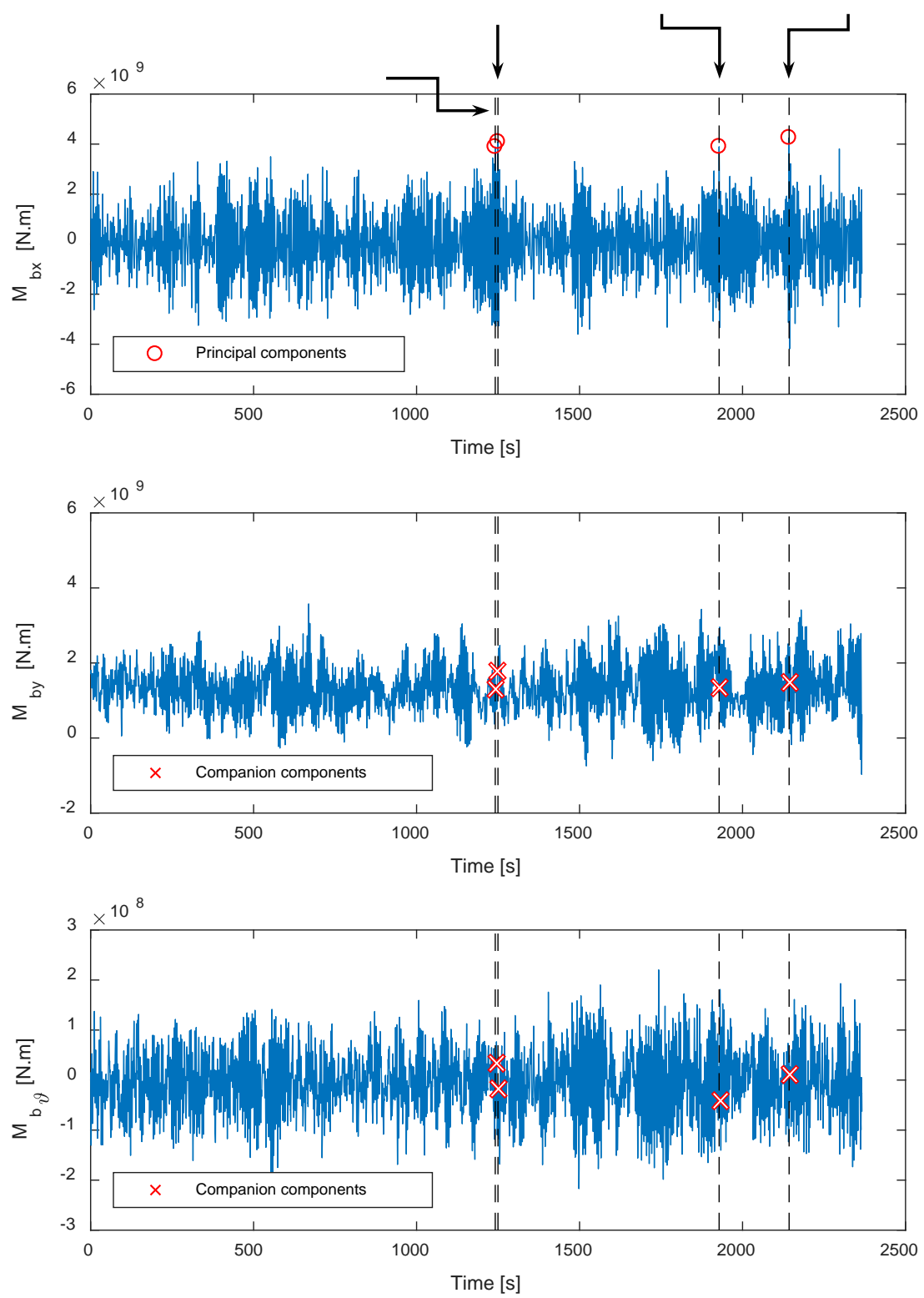

Figure 3. Sample data for three effective base moment components and load combinations $\left(n_{p i t}=4\right)$ 


\section{Analytical framework of DAD}

In this chapter, the basic structure of DAD is briefly described (Main and Fritz 2006; Park et al. 2017; Simiu et al. 2008; Simiu et al. 2003; Simiu and Yeo 2015; Spence 2009; Yeo 2010).

\subsection{Dynamic modeling of tall building including second-order effect}

A tall building can be represented by a multi-story model with lumped masses. A schematic building model is shown in Fig. 2. An $N$-story building has $N$ masses located at the center of mass of each floor. The building has three degrees of freedom (i.e., $x$-, $y$-, and $\vartheta$-axis) per floor.

The equation of motion for a forced, damped linear system is

$$
\mathbf{M} \ddot{\mathbf{u}}(t)+\mathbf{C} \dot{\mathbf{u}}(t)+\mathbf{K u}(t)=\mathbf{p}(t)
$$

where $\mathbf{u}(t)$ is the displacement response vector including displacement in the $x$ and $y$ axes and rotation in the $\vartheta$ axis. $\mathbf{M}, \mathbf{C}$, and $\mathbf{K}$, are mass, damping, and stiffness matrices, respectively. $\mathbf{p}(t)$ is the vector of external force time histories. Second-order effects (i.e., P- $\Delta$ and P- $\delta$ effects, a new feature in HR_DAD version 2.0) can be considered by replacing the stiffness matrix $\mathbf{K}$ by the matrix $\mathbf{K}-\mathbf{K}_{\mathbf{G}}$, where $\mathbf{K}_{\mathbf{G}}$ is the geometric stiffness matrix (Park and Yeo 2016).

When the linear system has classical damping whose square matrix, $\mathbf{C}$, is diagonal, Eq. 18 can be transformed into a set of uncoupled equations via modal analysis. Each equation of this set is analogous to, and can be solved as, an equation of motion for a single degreeof-freedom system. Note that the damping matrix $\mathbf{C}$ can be non-diagonal, depending on the distribution of damping in the system; in this case, the following classical modal analysis cannot be applied (Chopra 2016).

The total displacement of the structure $\mathbf{u}(t)$ can be represented as the sum of its modal contributions, and is dependent on the mode shapes $\boldsymbol{\Phi}$ and generalized coordinates $\mathbf{q}(t)$.

$$
\begin{aligned}
\mathbf{u}(t) & =\boldsymbol{\Phi q}(t) \\
& =\sum_{k=1}^{n} \phi_{k} q_{k}(t)
\end{aligned}
$$

where $\phi_{k}$ and $q_{k}$ are the mode shape and the generalized coordinate in the $k^{\text {th }}$ mode, respectively, and $n$ designates in this equation the number of modes of vibration being considered.

The natural frequencies $\boldsymbol{\omega}$, and modal shapes $\boldsymbol{\Phi}$ can be obtained from the equation of motion of the undamped, freely vibrating system by solving the equation

$$
\left[\mathbf{K}-\omega^{2} \mathbf{M}\right] \mathbf{\Phi}=0
$$

The generalized equation of motion in the $k^{\text {th }}$ mode is 


$$
m_{k}^{*} \ddot{q}_{k}(t)+c_{k}^{*} \dot{q}_{k}(t)+k_{k}^{*} q_{k}(t)=p_{k}^{*}(t)
$$

where the generalized mass $m_{k}^{*}$, damping $c_{k}^{*}$, stiffness $k_{k}^{*}$, and force $p_{k}^{*}$ are defined, respectively, as

$$
\begin{aligned}
& m_{k}^{*}=\phi_{k}^{T} \mathbf{M} \phi_{k} \\
& \mathrm{c}_{k}^{*}=\phi_{k}^{T} \mathbf{C} \phi_{k}=2 \xi_{n} m_{n}^{*} \\
& k_{k}^{*}=\phi_{k}^{T} \mathbf{K} \phi_{k}=m_{n}^{*} \omega_{n}^{2} \\
& p_{k}^{*}=\phi_{k}^{T} \mathbf{p}(t)
\end{aligned}
$$

The total displacements and accelerations are then obtained by Eq. 21. In this version of the software, the ramp-invariant filters are used as a dynamic solver. For details, see Appendix II.

The mass of each floor has three degrees of freedom (i.e., $x$-, $y$-, and $\vartheta$-axis) and is concentrated at the center of the mass on the floor. Definitions of story and floor used in this report are described in the Fig. 4. For any given wind direction, wind loads corresponding to the three coordinates are applied at the associated mass centers.

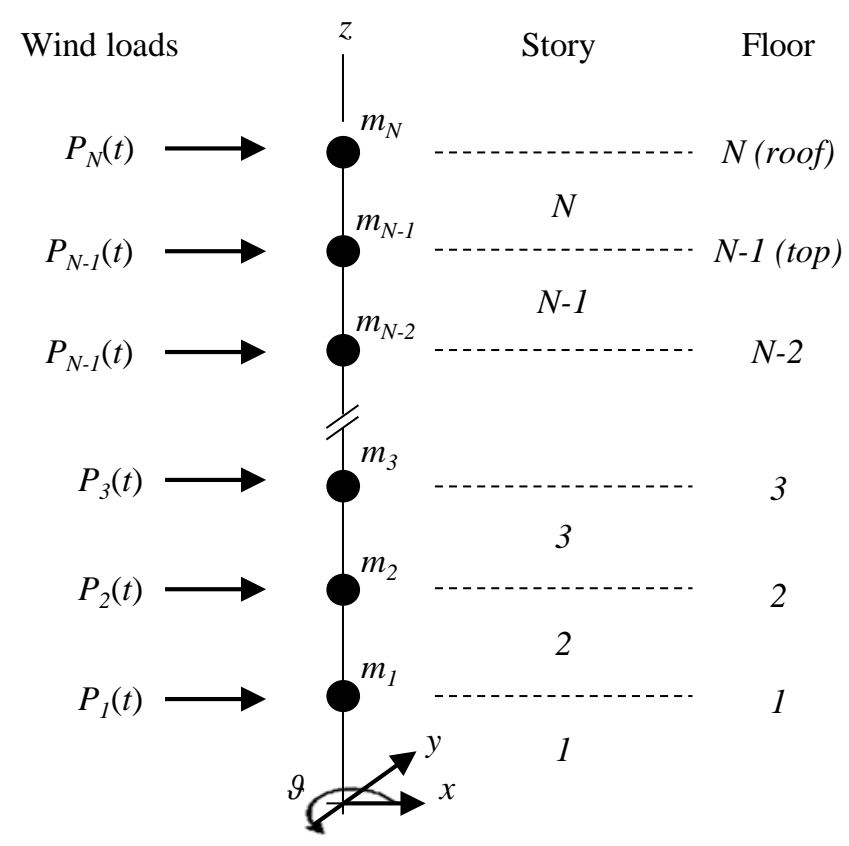

Figure 4. Schematic model of a tall building 


\subsection{Aerodynamic database and wind load modeling}

Pressure time histories can be obtained from wind tunnel tests or CFD simulations. This section focuses on load modeling based on wind tunnel tests.

The pressure data on the structure's envelope are generally expressed as nondimensional pressure coefficients $C_{p}$ based on the mean hourly wind speed $V_{H}$ at building roof height $H$ :

$$
C_{p}=\frac{p}{\frac{1}{2} \rho V_{H}^{2}}
$$

where $p$ is the net pressure relative to the atmospheric pressure and $\rho$ is the air density $\left(1.225 \mathrm{~kg} / \mathrm{m}^{3}\right.$ for $15^{\circ} \mathrm{C}$ air at sea level).

It is assumed that the pressure coefficients do not depend significantly upon Reynolds number and are therefore identical for the model and the prototype. Time series of prototype wind loads at each mass center along the building height can be calculated using pressures at measuring points and the associated tributary areas. From the similarity requirement for the reduced frequency $(f D / V)_{m}=(f D / V)_{p}$, where $f$ is sampling frequency, $D$ is the characteristic dimension of the structure, $V$ is a characteristic velocity, and the subscripts $m$ and $p$ stand for model and prototype, respectively, and it follows that the time interval $\Delta t_{p}$ at prototype scale is

$$
\Delta t_{p}=\frac{D_{p}}{D_{m}} \frac{V_{m}}{V_{p}} \Delta t_{m}
$$

where $D_{m} / D_{p}$ is the length scale, $V_{m} / V_{p}$ is the velocity scale, and $\Delta t_{m}$ is the time interval (i.e., the reciprocal of the sampling frequency $f_{m}$ ) at model scale. The time interval $\Delta t_{p}$ depends on the wind speed $V_{p}$.

Determining the floor wind loads requires the following input: the dimensions of the prototype; the model scale; the number of floors; the coordinates of all pressure tap locations; and the pressure coefficient time series. The software then performs the following operations:

1) Creation of virtual pressure taps at each edge of the model surface. The time series of the pressure coefficients at the virtual pressure taps are obtained by extrapolation from the time histories at the outermost and next to outermost pressure taps (see Fig. 5a). This operation is necessary because actual pressure taps cannot be placed at the structure's edges.

2) Generation of mesh for interpolation between pressure coefficients measured at the actual taps or assigned to the virtual taps (Fig. 5b). Each mesh element has dimensions $\Delta B$ by $\Delta H$, where $\Delta B=B /\left(2 n_{B}\right), \Delta H=H /(2 N)$, where $B$ is the building width, $H$ is the building height (inclusive, for buildings with parapets, of the parapet height, in which case the height of the uppermost mesh element is equal to the height of the parapet), and $n_{B}$ is the number of pressure taps in each pressure tap row. 
3) The user has the option of carrying out the interpolations by one of the following three methods supported by MATLAB: i) nearest, ii) linear, and iii) cubic. For details, see MATLAB documentation (www.mathworks.com/help/). Figure 6 shows the differences among the results obtained in those three interpolation methods as an example. The linear interpolation is typically sufficiently accurate for structural design purposes.

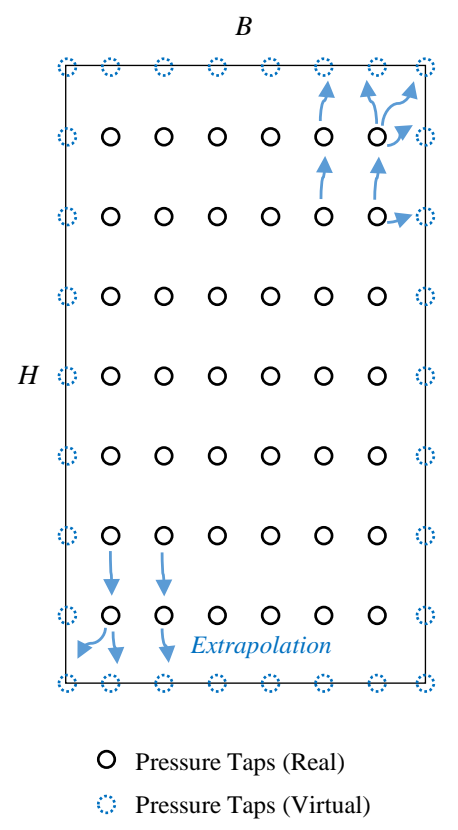

(a) Virtual taps on each edge of surface

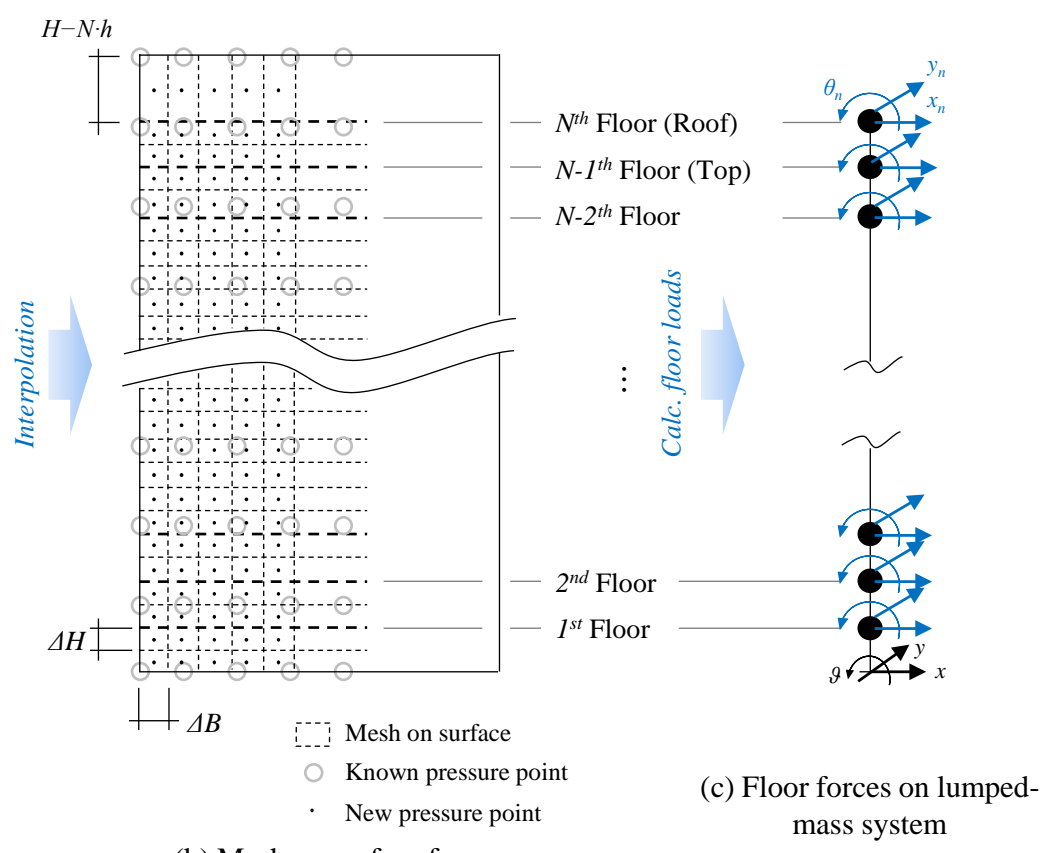

(b) Mesh on surface for interpolating pressures

Figure 5. Schematic drawings of interpolation mesh on model surface ( $h=$ floor height)
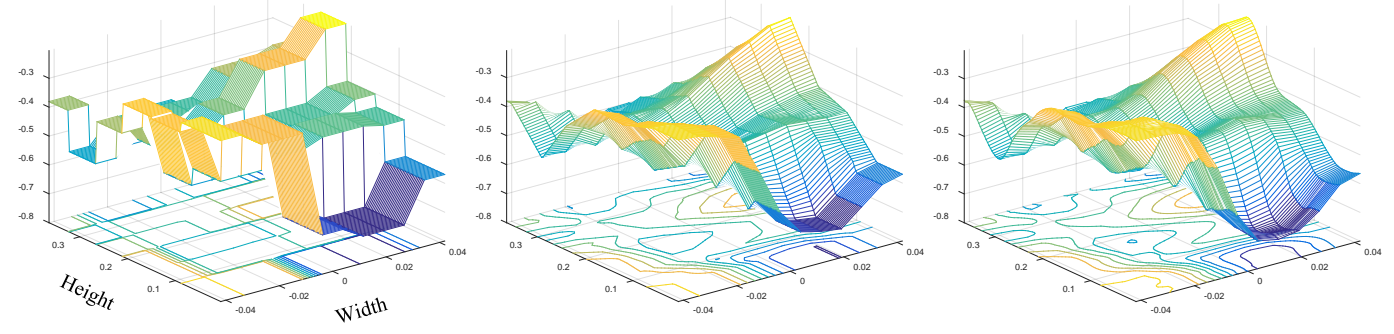

Figure 6. Illustration of results of nearest, linear, and cubic interpolation methods

4) Determination of wind loads acting at each floor mass center using the tributary areas shown in Fig. 7. The floor wind loads consist of forces along the two principal directions of the structure applied at the center of mass and a torsional moment about the center of mass. 


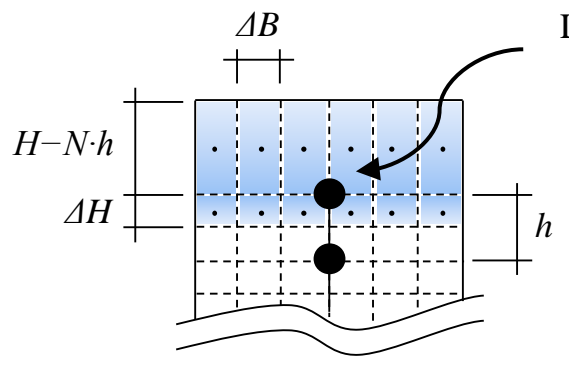

(a) Tributary area for $N^{\text {th }}$ floor (colored area on mesh)
Lumped-Mass

System

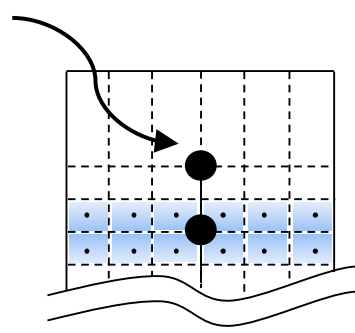

(b) Tributary area for middle floors

(colored area on mesh)

Figure 7. Tributary areas for calculation of floor wind loads

\subsection{Influence coefficients for determining internal forces}

Influence coefficients $f_{i j k}{ }^{N}$ are used to transform wind forces acting at the lumped mass of floor $N$ along the principal axes $q(q=1,2)$, and wind-induced torsional moments about the mass center of floor $N(q=3)$, into the wind effect of interest at cross section $j$ of member $i$. The influence coefficients of a building model can be obtained using a finite element analysis program. Note that if the second-order effect (i.e., P- $\Delta$ and P- $\delta$ effects) is considered, the influence coefficients should be changed accordingly.

The wind loads used for strength and serviceability designs are combined with dead and live loads using load combination cases specified by ASCE 7-16, Section 2.3:

For strength design,

$$
\begin{array}{ll}
1.2 D+1.0 L+1.0 W & \text { (LC1) } \\
0.9 D+1.0 W & \text { (LC2) }
\end{array}
$$

For serviceability design,

$$
1.0 D+1.0 L+1.0 W \quad(L C 3)
$$

where $D$ is the total dead load, $L$ is the live load, and $W$ is the wind load. Note that the load factor is not applied to the wind load because the DAD approach yields wind effects with the requisite mean recurrence intervals provided by the structural engineer. A different load factor for the live load may be used in Eq. 25 and 26 depending upon the engineer's judgment. The software provides an option that outputs the largest of the DCIs based on the various combinations being considered.

The software is coded to obtain internal forces at three sections of each structural member. For example, the three locations can be the member's two ends and midpoint. However, in some instances the critical sections, as defined by ACI 318 or AISC specifications, may occur at other locations. The influence coefficients required to obtain the internal forces must be calculated accordingly. 


\subsection{Response surface}

For each cross section of interest DAD determines response surfaces for a sufficient number of wind speeds and directions. The surfaces contain information on structural responses induced by combined gravity and wind loads. The structural responses of interest are demand-to-capacity indexes, inter-story drift, and peak accelerations. Details are provided in Section 5.

For the design of structural members, response surfaces pertaining to demand-tocapacity indexes are obtained for individual structural members (e.g., columns and beams) for a set of wind speeds and wind directions. Depending upon the wind climate, the set includes mean speeds from, say, $20 \mathrm{~m} / \mathrm{s}$ to $80 \mathrm{~m} / \mathrm{s}$ in increments of, say, $10 \mathrm{~m} / \mathrm{s}$, for wind directions from $0^{\circ}$ to $360^{\circ}$, typically in increments of $10^{\circ}$.

The index DCI for columns pertains to interaction equations for axial load and bending moments; for beams the index is based on the bending moment. Its counterpart for columns and beams is associated with interaction equations for shear forces and torsional moments. The subscripts $i$ and $j$ denote the identification of a member and a cross section of interest for the member, respectively.

For serviceability design, response surfaces for inter-story drift and peak accelerations are obtained along specified column lines throughout the height of the structure.

Using interpolation techniques, the structural response can be obtained for any wind direction and speed within the specified ranges. As noted earlier the directions for which the responses are provided in the response surfaces are referenced to the principal axes of the building (i.e., the $x$ - and $y$-axis in Fig. 9). The response surfaces are independent of wind climate. Figure 8 represents an example of the response surfaces of DCI for a steel column member (Park and Yeo 2016).

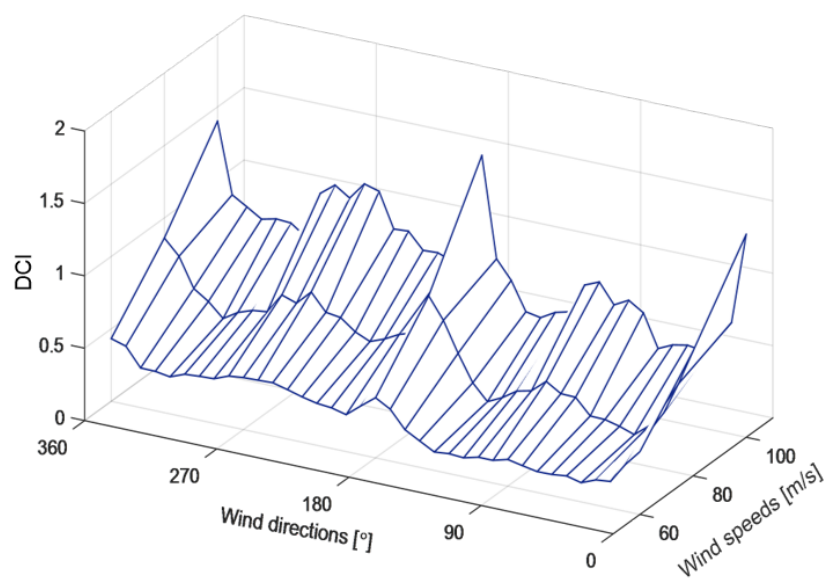

Figure 8. Example of response surface 


\subsection{Peak directional response}

Structural responses to directional wind are obtained by using the directional wind speeds included in the wind climatological database in conjunction with the response surfaces. The wind climatological database for the calculations presented in this report is based on a wind speed dataset of 999 simulated hurricanes for 16 directions (Batts et al. 1980). The wind climatological database from which the dataset is extracted is publicly available at www.nist.gov/wind.

The database sets on the NIST website consist of one-minute mean hurricane wind speeds in knots at $10 \mathrm{~m}$ above the ground over terrain with open exposure near the coast line $(1 \mathrm{knot} \approx 0.5144 \mathrm{~m} / \mathrm{s})$. The 16 wind directions are from $22.5^{\circ}$ to $360^{\circ}$ in $22.5^{\circ}$ clockwise increments from the north. Those speeds are converted to mean hourly speeds $(\mathrm{m} / \mathrm{s})$ at the rooftop of the building corresponding to the terrain exposure at the site. Providing the original and converted wind climatological database is incumbent on the wind engineering consultant.

For non-hurricane data, a procedure for generating time series of directional extreme winds is required. First, an algorithm developed by Yeo (2014) is used to estimate the parameters of the distributions of the directional wind speeds, given that the size of the data sample within one or more directional sectors can in some instances be too small for statistical inference purposes. Once the distribution parameters are estimated, a simple Monte Carlo procedure is used for data generation.

DAD also accounts for building orientation, defined by the angle $\alpha_{0}$ in Fig. 9. Because the wind direction $\theta_{w}$ angle in the response database is not identical to the wind direction angle $\alpha$ in the climatological database (Fig. 9), each wind direction in the wind climatological database should be rotated by the orientation angle $\alpha_{0}$.

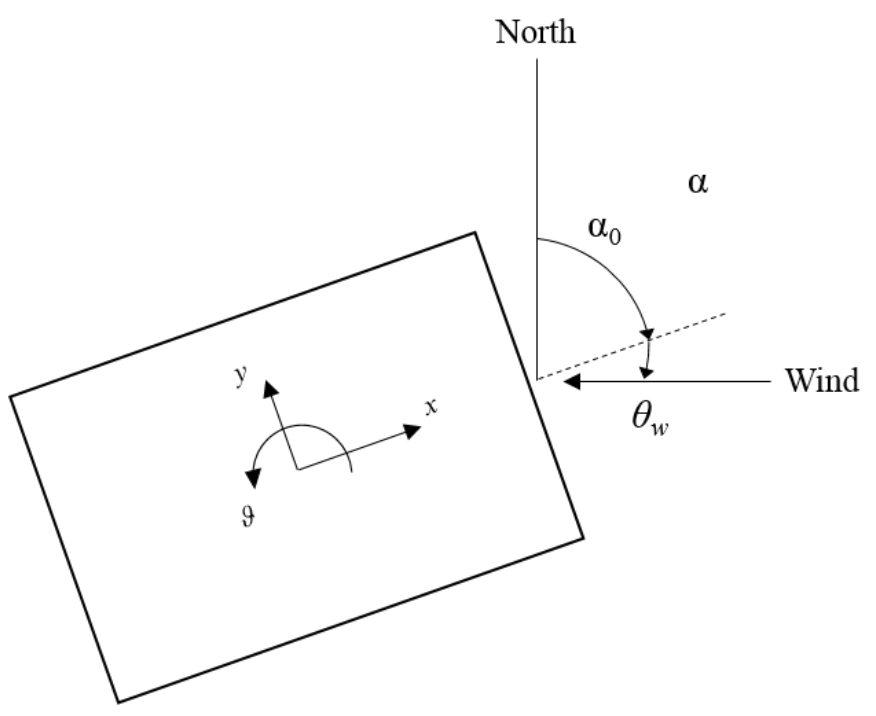

Figure 9. Wind directions 


\subsection{Peak wind effects with specified MRIs in regular and mixed wind climates}

The time series of peak wind effects induced by each storm event in the wind climatological database is obtained from the matrix of directional wind speeds [ $U_{r s}$ ]. First the entries $U_{r s}$ are replaced in that matrix by the quantities $\operatorname{DCI}^{p k}\left(U_{r s}\right)$ taken from the response surface. Second, transform the matrix $\left[\mathrm{DCI}^{p k}\left(U_{r s}\right)\right]$ into the vector $\left[\mathrm{DCI}^{p k}\left(U_{r}\right)\right]^{\mathrm{T}}$ where $\mathrm{T}$ denotes transpose, by disregarding in each row $r$ all DCIs lower than $\mathrm{DC}^{p k}\left(U_{r}\right)$. Third, rank-order the quantities $\operatorname{DCI}^{p k}\left(U_{r}\right)$ and use non-parametric statistics in conjunction with the mean annual rate of storm arrival $\lambda$, to obtain the quantities $\operatorname{DCI}^{p k}(\bar{N})$. The MRI of the $k^{\text {th }}$ highestranking quantity $\mathrm{DCI}^{p k}$ is

$$
\bar{N}_{k}=\left[1-\exp \left(-\frac{\lambda k}{n+1}\right)\right]^{-1}
$$

where $n$ denotes the number of storm events. For $n>30$, say,

$$
\bar{N}_{k} \approx \frac{n+1}{\lambda k}
$$

We now consider the case of multiple hazards, for example synoptic and thunderstorm wind speeds, or hurricanes and non-hurricane winds.

Example. Assume that the mean annual rates of synoptic storm and thunderstorm arrival at the location of interest are $\lambda_{s}=4 /$ year and $\lambda_{t}=3.5 /$ year. Table 1 lists rank-ordered DCIs induced in a structural member by $n_{s}=10,000$ synthetic synoptic storms and $n_{t}=10,000$ thunderstorms.

Table 1. Rank-ordered DCIs induced by synoptic storm and thunderstorm winds

\begin{tabular}{cccc}
\hline \multirow{2}{*}{$\begin{array}{c}\text { DCIs induced by synoptic storms } \\
\text { Rank }\end{array}$} & \multicolumn{2}{c}{ DCIs induced by thunderstorms } \\
& & Rank & DCI \\
\hline & & 1 & 1.34 \\
& & 3 & 1.30 \\
1 & 1.22 & 4 & 1.26 \\
2 & 1.16 & 5 & 1.23 \\
& & & 1.21 \\
4 & 1.10 & 6 & 1.18 \\
& 1.04 & 7 & 1.10 \\
5 & 1.01 & 8 & 1.02 \\
& & 9 & 1.01 \\
& 0.99 & 10 & 0.98 \\
\hline
\end{tabular}


The MRI of DCIs $>1.00$ induced by synoptic storms is $\bar{N}_{s}=\left(n_{s}+1\right) /\left(k_{s} \lambda_{s}\right)=$ $(10,001 /(5 \times 4)=500$ years, so the probability that the DCI induced by synoptic winds is greater than 1.00 is $1 / 500$ in any one year. Similarly, the probability that the DCI induced by thunderstorm winds is greater than 1.00 is $1 /[10,001 /(9 \times 3.5)]=1 / 317$ in any one year. The annual probability that the DCI induced by synoptic winds or by thunderstorms is greater than 1.00 is $1 / 500+1 / 317=0.00516$. This corresponds to an MRI of the occurrence of the event DCI $>1.00$ equal to $\bar{N} \approx 1 / 0.00516=194$ years (Simiu and Yeo 2015; Yeo 2011).

\subsection{Adjustment of demand-to-capacity indexes}

Section 31.4.3 of the ASCE 7-16 Standard requires that, "Loads for the main wind force resisting system determined by wind tunnel testing shall be limited such that the overall principal loads in the $x$ and $y$ directions are not less than 80 percent of those that would be obtained from Part 1 of Chapter 27... The overall principal load shall be based on the overturning moment for flexible buildings and the base shear for other buildings. ... The limiting values of 80 percent may be reduced to 50 percent for the main wind force resisting system ... if ...there are no specific influential buildings or objects within the detailed proximity model; (2) loads and pressures from supplemental tests for all significant wind directions in which specific influential buildings or objects are replaced by the roughness representative of the adjacent roughness condition, but not rougher than Exposure B, are included in the test results."

If $M_{o}{ }^{D A D} / M_{o}{ }^{A S C E} 7<0.8$, where $M_{0}$ denotes overturning moment, the software offers the option of satisfying the ASCE 7-16 Section 31.4.3 requirement by multiplying the DCI by the factor $\gamma$ as follows:

$$
\begin{aligned}
& D C I^{*}=\gamma D C I \\
& \gamma=\frac{0.8}{M_{o}^{D A D} / M_{o}^{A S C E 7}} \text { or } \gamma=\frac{0.5}{M_{o}^{D A D} / M_{o}^{A S C E 7}}
\end{aligned}
$$

where $M_{o}{ }^{D A D}$ and $M_{o}{ }^{A S C E 7}$ may either include only $1^{\text {st }}$ order terms, or both $1^{\text {st }}$ and $2^{\text {nd }}$ order terms, depending on the analysis method chosen by the users, obtained from DAD and Section 27, ASCE 7-16, respectively, and $\gamma$ is the index adjustment factor. If the moment in DAD is not less than $80 \%$ or $50 \%$ of the ASCE 7-16 value, the index need not be modified. 


\subsection{Compliance with design criteria}

The output of the software includes the values of the peak responses (i.e., demand-tocapacity index, inter-story drift, and acceleration) corresponding to the specified MRIs $\bar{N}$.

The peak demand-to-capacity indexes, accounting if desired for ASCE 7 limitations on overturning moments, must be equal to or less than unity for every structural member, i.e., $D_{C I}^{*}(\bar{N}) \leq 1$. If these relations are satisfied, the structural members are adequately designed for axial force and bending moments, and for shear force and torsional moments, respectively. When more than one load combination is considered in the design procedure (Eqs. 9), the DCI of a member is determined as the larger value among DCIs calculated from load combinations.

The peak inter-story drift ratio (defined as the ratio of drift to story height, see Eq. 36) is not limited by ASCE 7-16 requirements. However, the ASCE 7-16 Commentary suggests limits on the order of 1/600 to 1/400 (see Section CC.1.2 in ASCE 7-16). If the peak inter-story drift ratio with a 20-year MRI is equal to or less than the suggested limit, the design is adequate for inter-story drift. This criterion may be changed as judged appropriate by the building owner and designer.

Peak resultant accelerations at the top floor are also not limited by ASCE 7-16. This study assumes a limit of $25 \mathrm{mg}$ (milli-g) for a 10-year MRI for office buildings (Isyumov et al. 1992). If peak acceleration for MRI = 10 year is not greater than this limit the design is adequate for peak acceleration.

The procedure outlined in Sections 4.1 to 4.7 is repeated as needed with a modified structural design (e.g., by resizing members or by installing dampers) until the results satisfy the design criteria. 


\section{Structural responses}

The loads induce in the structure three types of response considered in design: demand-to capacity indexes for steel members (Section 5.1) or reinforced concrete members (Section 5.2), inter-story drift (Section 5.3), and accelerations (Section 5.4).

\subsection{Demand-to-capacity indexes for steel members}

The demand-to-capacity index is a quantity used to measure the adequacy of a structural member's strength. The subscripts indicate the member $i$ and the cross section $j$ of that member. In general, this index is defined as a ratio or sum of ratios of the required internal force to the respective available capacity. The design strength in DAD_ESWL is based on the AISC Specification for Structural Steel Buildings (ANSI/AISC 2010). A DCI larger than unity indicates inadequate design of a structural member. The software employs up to three demand-to-capacity indexes per member type: (i) for axial and/or flexural loads $\left(D C I^{P M}\right)$, (ii) for shear $\left(D C I^{V}\right)$, and (iii) for combined axial, flexural, shear, and torsion, applicable to Hollow Structural Section (HSS) only. Figure 10 shows the flow chart that describes the process of determining DCIs for steel members.

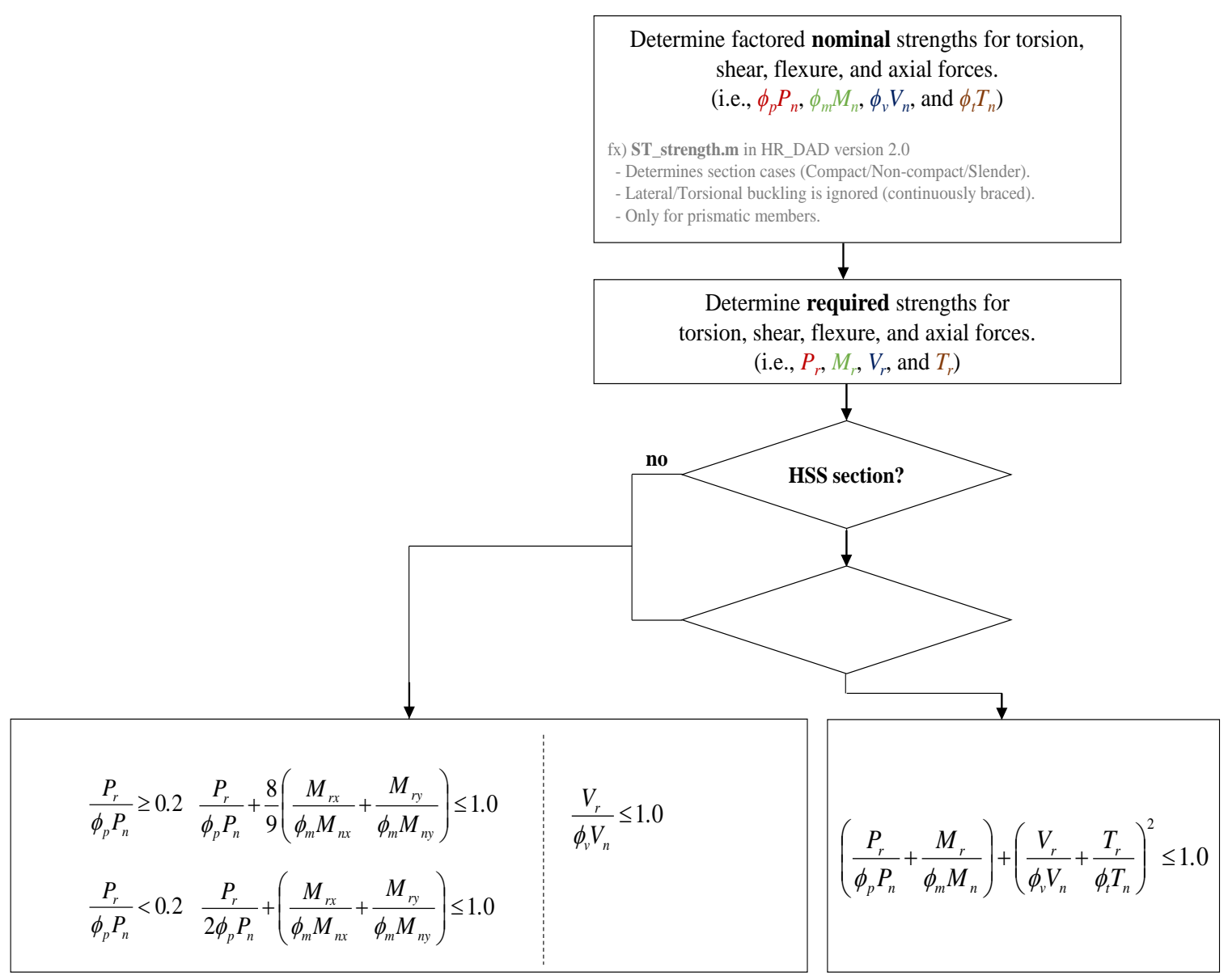

Figure 10. Flowchart of determining DCIs for steel members 
In the figure, the symbols $P, M, V$, and $T$ represent axial force, bending moment, shear force, and torsion, respectively. The subscripts $n$ and $r$ are design nominal strengths and required strengths, respectively. $\phi_{i}$ is the resistance factor for each type of strength $(i=p$, $m, v$, and $t$ ). When the required torsional strength is less than or equal to $20 \%$ of the available torsional strength, the interaction of torsion, shear, flexure and/or axial force for HSS (Hollow Structural Section) members shall be determined by the equations in box A and the torsional effects shall be ignored. When the required torsional strength exceeds $20 \%$ of the available torsional strength, the interaction of torsion, shear, flexure and/or axial force shall be limited, at the point under consideration, by the equation in box C.

\subsection{Demand-to-capacity indexes for $\mathrm{RC}$ members}

The design strength in DAD_ESWL is based on the Building Code Requirements for Structural Concrete and Commentary 318-14 (ACI 2014). The index DCI ${ }^{P M}$ pertains to bending moments in beams:

$$
D C I^{P M}=\frac{M_{u}}{\phi_{m} M_{n}} \quad \text { (for beams) }
$$

where $M_{u}$ is the factored bending moment at the section, $M_{n}$ is the nominal moment and axial strengths at the section, and $\phi_{m}$ is the reduction factors for flexural and axial strengths.

For columns, the index pertains to the interaction between the axial force and the bending moments about the cross section's principal axes. The interaction is defined by the position of the factored axial force and the bending moments in the axial load-bending moments interaction diagram (called the PM diagram or PMM surface). The software generates a biaxial interaction strength surface to determine the demand-to-capacity index for that member. The interaction strength surface is defined by a series of PMM interaction curves (i.e., axial force $P$ and bending moments $M_{2}$ and $M_{3}$, where $M_{2}$ and $M_{3}$ are the bending moments about the $y$ - and z-axis, respectively, see Fig. 11) corresponding to equal angular increments from 0 to $360^{\circ}$. Each $P M M$ interaction curve is numerically described by a series of discrete points connected by straight lines. The coordinates of these points are determined by rotating a plane of linear strain about the neutral axis on the section of the member. By default, 101 points are used to define a PMM interaction curve. Figure 11 shows planes of linear strain for neutral axis in two orientation angle cases. The orientation angle $\theta$ is the angle between the $z$-axis and the neutral axis. Note that the upper plot is for $\theta=0^{\circ}$. The angle $\theta$ can be transformed to the angle $\beta$ between the $y$-axis and the eccentric load $P$. Equations 29 and 30 define the angles $\theta$ and $\beta$ :

$$
\begin{aligned}
& \theta=\tan ^{-1}\left(\frac{I_{y}}{I_{z}} \tan \beta\right) \\
& \beta=\tan ^{-1}\left(\frac{M_{2}}{M_{3}}\right)
\end{aligned}
$$




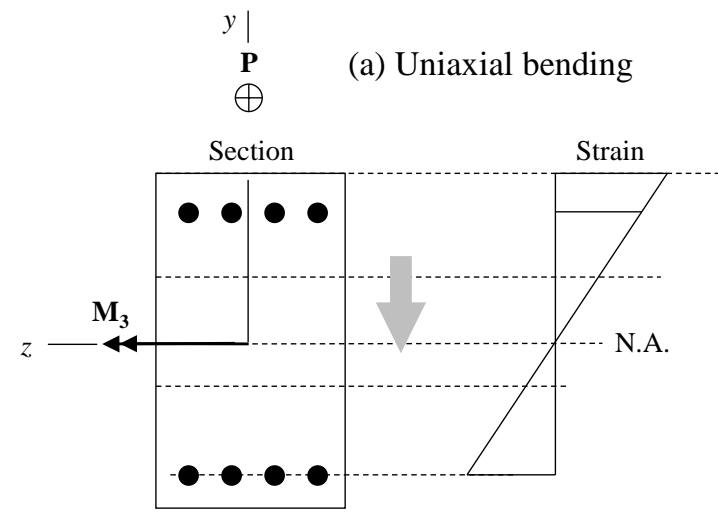

101 points (analyze 101 neutral axes per one PMM curve)

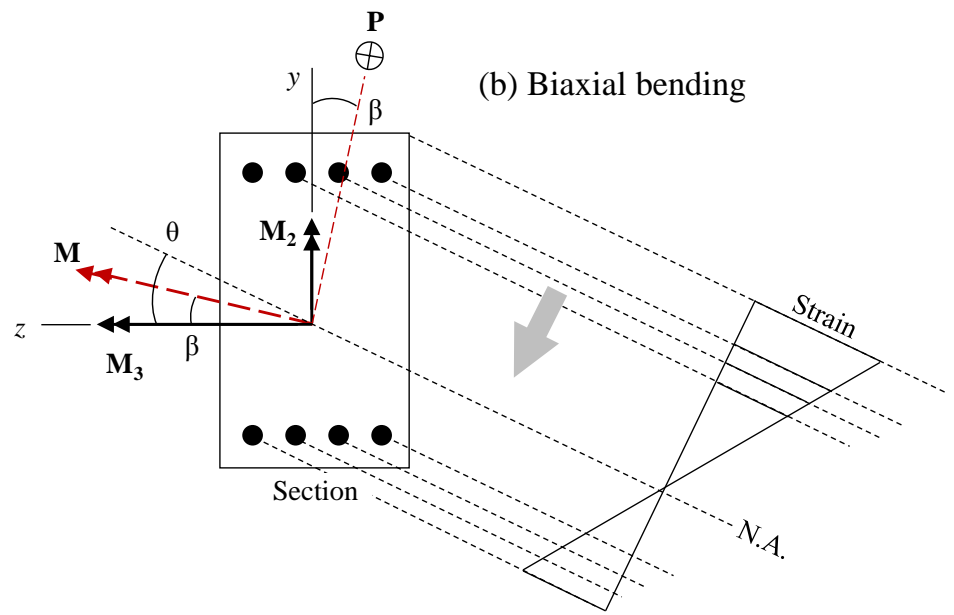

101 points (analyze 101 neutral axes per one PMM curve)

Figure 11. Reinforced concrete section subjected to biaxial moments with varying planes of linear strain

Based on Section 22.2 of ACI 318-14 (ACI 2014), the software uses the requirements of force equilibrium and strain compatibility to determine the nominal axial force and bending moment strengths $\left(P_{n}, M_{2 n}\right.$, and $\left.M_{3 n}\right)$ of the member. For the inclined neutral axes, four types of the equivalent compressive stress block are used in the software (Park and Paulay 1975). The nominal strengths are then multiplied by the appropriate strength reduction factors $\phi$. The biaxial interaction surface generated by the software considers the effect of the strength reduction factors. The strength reduction factors for moment, axial force, or combined moment and axial force are defined by Eq. 31 (ACI 318-14, Table 21.2.2):

$$
\phi=\left\{\begin{array}{ccc}
\phi_{c} & \varepsilon_{t} \leq \varepsilon_{t y} & \text { Compression - controlled } \\
\phi_{c}+\left(\phi_{t}-\phi_{c}\right) \frac{\left(\varepsilon_{t}-\varepsilon_{t y}\right)}{\left(0.005-\varepsilon_{t y}\right)} & \varepsilon_{t y}<\varepsilon_{t}<0.005 & \text { Transition } \\
\phi_{t} & \varepsilon_{t} \geq 0.005 & \text { Tension - controlled }
\end{array}\right.
$$


where, $\phi_{t}$ is 0.9 for tension-controlled sections, $\phi_{c}$ is 0.65 for compression-controlled sections (0.75 for spirals conforming, see Section 25.7.3 of ACI 318-14), $\varepsilon_{t}$ is the net tensile strain, and $\varepsilon_{t y}$ is defined as $f_{y} / E_{s}$ for deformed reinforcement where $f_{y}$ is the yield strength of reinforcement and $E_{s}$ is the modulus of elasticity of reinforcement. Figure 12 shows an example of PMM surface for an RC column section (500 mm of width, $800 \mathrm{~mm}$ of height) obtained in the DAD_ESWL. Appendix III summarizes comparison of the results with those from a commercial program.

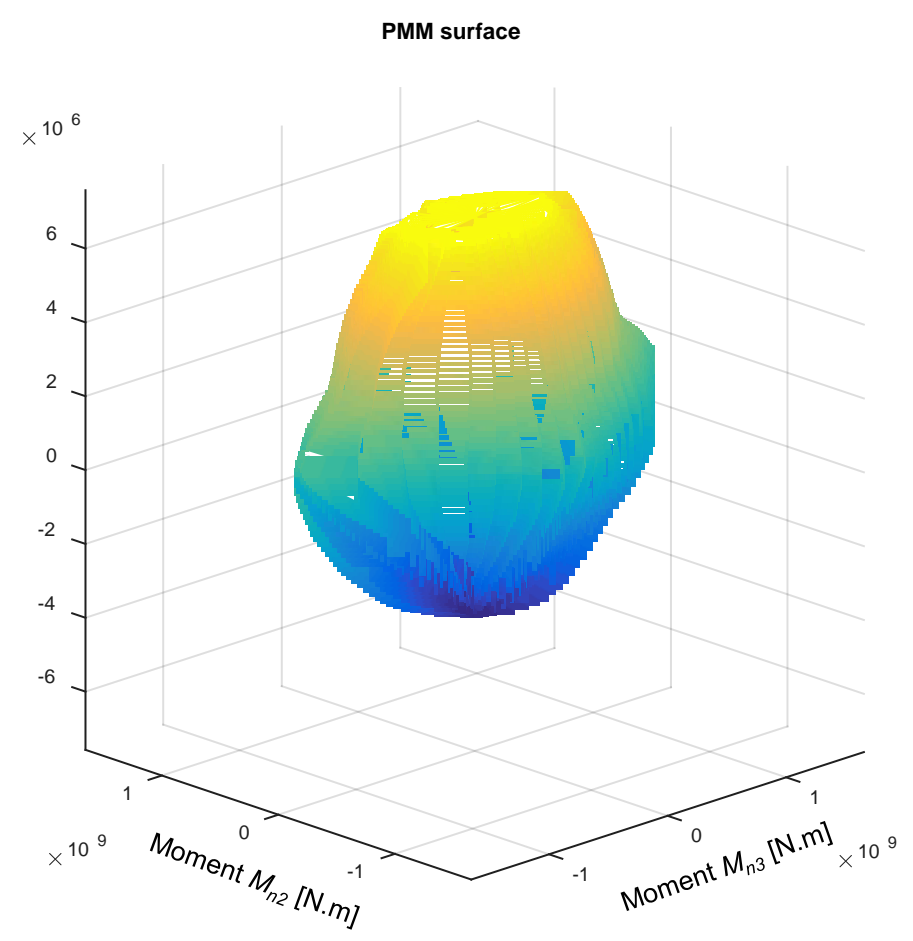

Figure 12. PMM interaction surface for RC column section

When the factored internal forces $\left(P_{r}, M_{2 r}\right.$, and $\left.M_{3 r}\right)$ on each member are calculated from the dynamic analysis of DAD_ESWL, they shall be placed on the two-dimensional interaction diagram which corresponds to the appropriate angle $\beta$ defined by $M_{2 r}$ and $M_{3 r}$ (Eq. 32) from the three-dimensional interaction surface. Figure 13 represents twodimensional interaction diagram at a given angle $\beta$ from the PMM surface. The demandto-capacity index can be defined as

$$
D C I^{P M}=\frac{P M M_{u}}{\phi P M M_{n}}=\frac{\overline{O D}}{\overline{O C}} \leq 1
$$

where $P M M_{u}$ is the factored bending moment and axial force at the section, $P M M_{n}$ is the nominal bending moment and axial force at the section, $\phi$ is the strength reduction factors mentioned above, $\overline{O D}$ is the distance from point $\mathrm{O}$ to point $\mathrm{D}$ (demand) and $\overline{O C}$ is the distance from point $\mathrm{O}$ to point $\mathrm{C}$ (capacity). If the point $\mathrm{D}$ lies within the interaction 
diagram, the demand-to-capacity index is less than unity. Otherwise the section should be redesigned for $D C I^{P M} \leq 1$.

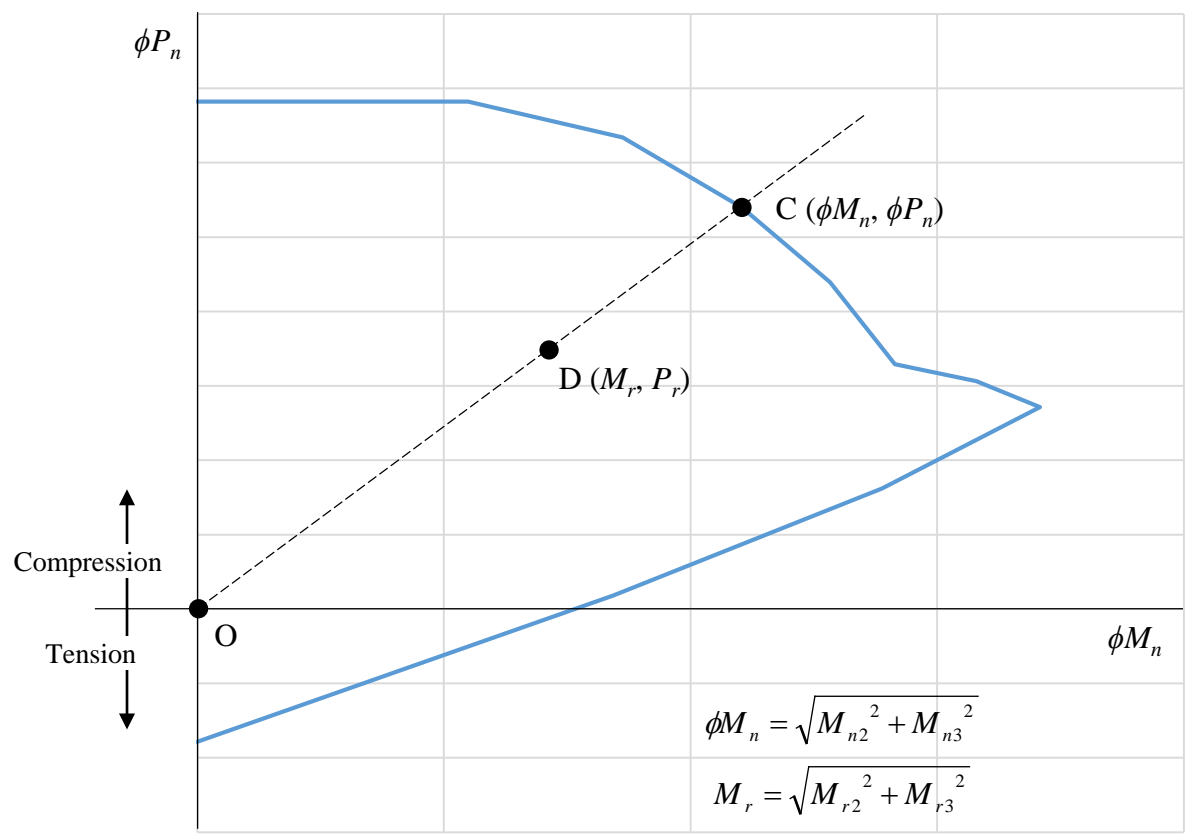

Figure 13. Definition of demand-to-capacity index for RC section

The index $D C I^{V T}$ is associated with interaction equations for bi-axial shear forces and torsional moment for columns and beams:

$$
D C I^{V T}=\frac{\sqrt{V_{u x}^{2}+V_{u y}^{2}+\left(\frac{T_{u} p_{h} b_{w} d}{1.7 A_{o h}^{2}}\right)}}{\phi_{v}\left(V_{c}+V_{s}\right)}
$$

where $V_{c}$ and $V_{s}$ are the nominal shear strengths provided by concrete and by reinforcement, respectively, $V_{u x}$ and $V_{u y}$ are the shear force in the $x$ - and $y$-axis, respectively, $T_{u}$ is the torsional moment, $\phi_{v}$ is the reduction factors for shear strengths, $p_{h}$ is the perimeter enclosed by the centerline of the outermost closed stirrups, $A_{o h}$ is the area enclosed by the centerline of the outermost closed stirrups, $b_{w}$ is the width of the member, and $d$ is the distance from extreme compression fiber to the centroid of longitudinal tension reinforcement. 


\subsection{Inter-story drift ratio}

The time-series of the inter-story drift ratios at the $i^{\text {th }}$ story, $d_{i, x}(t)$ and $d_{i, y}(t)$, corresponding to the $x$ - and y-axis, are:

$$
\begin{aligned}
& d_{i, x}(t)=\frac{\left[x_{i}(t)-D_{i, y} \vartheta_{i}(t)\right]-\left[x_{i-1}(t)-D_{i-1, y} \vartheta_{i-1}(t)\right]}{h_{i}} \\
& d_{i, y}(t)=\frac{\left[y_{i}(t)-D_{i, x} \vartheta_{i}(t)\right]-\left[y_{i-1}(t)-D_{i-1, x} \vartheta_{i-1}(t)\right]}{h_{i}}
\end{aligned}
$$

where $x_{i}(t), y_{i}(t)$, and $\vartheta_{i}(t)$ are the displacements and rotation at the mass center at the $i^{\text {th }}$ floor, $D_{i, x}$ and $D_{i, y}$ are distances along the $x$ - and $y$-axis from the mass center on the $i^{\text {th }}$ floor to the point of interest on that floor (Fig. 14), and $h_{i}$ is the $i^{\text {th }}$ story height between mass centers of the ith and the $i-1^{\text {th }}$ floor.

\subsection{Floor acceleration}

The time-series of resultant acceleration at each floor, $a_{i, r}(t)$ are yielded in DAD_ESWL by the expression:

$$
a_{i, r}(t)=\sqrt{\left[\ddot{x}_{i}(t)-D_{i, y} \ddot{\vartheta}_{i}(t)\right]^{2}+\left[\ddot{y}_{i}(t)+D_{i, x} \ddot{\vartheta}_{i}(t)\right]^{2}}
$$

where accelerations $\ddot{x}_{i}(t), \ddot{y}_{i}(t)$, and $\ddot{\vartheta}_{i}(t)$ of the mass center at the $i^{\text {th }}$ floor pertain to the $x$-, $y$-, and $\vartheta$ - (i.e., rotational $z$ ) axis, and $D_{i, x}$ and $D_{i, y}$ are the distances along the $x$ - and $y$ axis from the mass center to the point of interest on the $i^{\text {th }}$ floor.

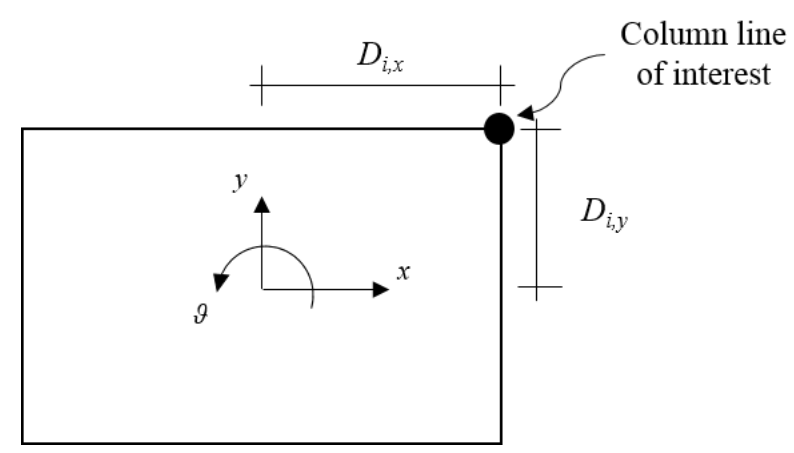

Figure 14. Position parameters at floor $i$ for inter-story drift ratio and acceleration 


\section{Summary}

This report presents the software DAD_ESWL for the structural design considering the equivalent static wind loads (ESWLs) as well as the database-assisted design (DAD) of steel and reinforced concrete buildings.

The DAD procedure makes use of simultaneous time series of aerodynamic pressure data obtained in the wind tunnel or by CFD methods. It determines effective lateral load time histories at the mass center of each floor, and (i) peak demand-to-capacity indexes (DCI) for axial force and moments and for shear force and torsion, and (ii) displacement and acceleration time histories, for any specified mean recurrence interval.

Like DAD, the ESWL procedure requires the wind engineer to provide (i) wind climatological data at the building site, and (ii) time series of pressure coefficients measured simultaneously at multiple taps; once these two tasks are completed, the design process is fully the responsibility of the structural engineer.

For both the ESWLs and DAD procedures, once the requisite wind climatological and aerodynamic data are provided by the wind engineer, the structural engineer is in full control of the design process. This allows the iterative design process to proceed smoothly and in a timely fashion until the calculated DCIs are close to unity.

The report also includes a detailed user's manual for the software DAD_ESWL (see Appendix I). A case study that illustrates the design of a 47-story steel building is presented in a companion paper (Park et al. 2018). 


\section{References}

ACI (2014). Building code requirements for structural concrete, ACI 318-14, American Concrete Institute, Farmington Hills, MI.

ANSI/AISC (2010). Specifications for Structural Steel Buildings, AISC 360-10, American Institute of Steel Construction, Chicago, Illinois.

ASCE (2016). Minimum design loads for buildings and other structures, ASCE 7-16, American Society of Civil Engineers, Reston, VA.

Batts, M. E., Russell, L. R., and Simiu, E. (1980). "Hurricane wind speeds in the United States." Journal of the Structural Division-ASCE, 106(10), 2001-2016.

Boggs, D. and Lepage, A. (2006). "Wind tunnel methods." Special Publication, 240, 125142.

Canada, N. R. C. o. (1971). Canadian Structural Design Manual, Supplement No. 4 to the National Building Code of Canada, Ontario.

Chopra, A. K. (2016). Dynamics of Structures, 4th ed., Pearson.

Coffman, B. F., Main, J. A., Duthinh, D., and Simiu, E. (2010). "Wind Effects on LowRise Metal Buildings: Database-Assisted Design versus ASCE 7-05 Standard Estimates." Journal of Structural Engineering, 136(6), 744-748.

CSI (2015). Integrated Finite Element Analysis and Design of Structures, v.17.

Fritz, W. P., Bienkiewicz, B., Cui, B., Flamand, O., Ho, T. C., Kikitsu, H., Letchford, C. W., and Simiu, E. (2008). "International Comparison of Wind Tunnel Estimates of Wind Effects on Low-Rise Buildings: Test-Related Uncertainties." Journal of Structural Engineering, 134(12), 1887-1890.

Garber, J., Browne, M. T. L., Xie, J., and Kumar, K. S. (2007). "Benefits of the pressure integration technique in the design of tall buildings for wind." ICWE12 Cairns.

Ho, T. C. E., Jeong, U. Y., and Case, P. (2014). "Components of wind -tunnel analysis using force balance test data." Wind and Structures, 18(4), 347-373.

Huang, G. and Chen, X. (2007). "Wind load effects and equivalent static wind loads of tall buildings based on synchronous pressure measurements." Engineering Structures, 29(10), 2641-2653.

Isyumov, N., Fediw, A. A., Colaco, J., and Banavalkar, P. V. (1992). "Performance of a tall building under wind action." Journal of Wind Engineering and Industrial Aerodynamics, 42(1), 1053-1064.

Main, J. A. and Fritz, W. P. (2006). "Database-Assisted Design for Wind: Concepts, Software, and Examples for Rigid and Flexible Buildings." NIST Building Science Series 180, National Institute of Standards and Technology, Gaithersburg, MD.

Melbourne, W. H. (1980). "Comparison of measurements on the CAARC standard tall building model in simulated model wind flows." Journal of Wind Engineering and Industrial Aerodynamics, 6(1), 73-88.

Park, R. and Paulay, T. (1975). Reinforced Concrete Structures, 1st ed., Wiley.

Park, S. and Yeo, D. (2016). Database-Assisted Design and second-order effects on the wind-induced structural behavior of high-rise buildings. National Institute of Standards and Technology, Gaithersburg, MD, USA. 
Park, S. and Yeo, D. (2018). "Second-Order Effects on Wind-Induced Structural Behavior of High-Rise Steel Buildings." Journal of Structural Engineering, 144(2), 04017209.

Park, S., Yeo, D., and Simiu, E. (2017). Database-Assisted Design for Wind of Steel and Reinforced Concrete Structures: Concepts, Software, and User's Manual. National Institute of Standard and Technology, Gaithersburg, MD, USA.

Park, S., Yeo, D., and Simiu, E. (2018). "Equivalent static wind loads VS. DatabaseAssisted Design of tall buildings: An assessment." Engineering Structures, In review.

Simiu, E., Gabbai, R. D., and Fritz, W. P. (2008). "Wind-induced tall building response: A time-domain approach." Wind and Structures, 11(6), 427-440.

Simiu, E., Sadek, F., Whalen, T. A., Jang, S., Lu, L.-W., Diniz, S. M. C., Grazini, A., and Riley, M. A. (2003). "Achieving safer and more economical buildings through database-assisted, reliability-based design for wind." J. Wind Eng. Ind. Aerodyn., 91, 1587.

Simiu, E. and Yeo, D. (2015). "Advances in the design of high-rise structures by the wind tunnel procedure: Conceptual framework." Wind and Structures, 21(5), 489-503.

Smallwood, D. O. (1980). "An improved recursive formula for calculating shock response spectra." Shock and Vibration Bulletin, 51(2), 211-217.

SOM (2004). WTC wind load estimates, outside experts for baseline structural performance Appendix D. Skidmore, Owings and Merrill LLP, Chicago, IL, 13 April 2004 (wtc.nist.gov), also reproduced as Appendix to NIST Technical Note 1655, "Toward a standard on the wind tunnel method" (2009) by E. Simiu, pp. A1-A7 (www.nist.gov/wind).

Spence, S. M. J. (2009). "High-rise database-assisted design 1.1 (HR_DAD_1.1): Concepts, software, and examples." NIST Building Science Series 181, National Institute of Standards and Technology, Gaithersburg, MD.

Sterns, S. D. (1975). Digital Signal Analysis, ed., Hayden Book Company, Inc., Rochelle Park, New Jersey.

White, D. W. and Hajjar, J. F. (1991). "Application of second-order elastic analysis in LRFD: research to practice." Engineering Journal, 28(4), 133-148.

Yeo, D. (2010). "Database-Assisted Design of high-rise reinforced concrete structures for wind: Concepts, software, and application." NIST Technical Note 1665, National Institute of Standards and Technology, Gaithersburg, MD.

Yeo, D. (2011). "Database-Assisted Design for High-Rise Structures in Mixed Extreme Wind Climates." 13th International Conference on Wind Engineering, Amsterdam, Netherlands, Jul. 10-15.

Yeo, D. (2013). "Multiple Points-In-Time Estimation of Peak Wind Effects on Structures." Journal of Structural Engineering, 139(3), 462-471.

Yeo, D. (2014). "Generation of Large Directional Wind Speed Data Sets for Estimation of Wind Effects with Long Return Periods." Journal of Structural Engineering, 140(10), 04014073. 


\title{
Appendix I. User's Manual for DAD_ESWL v1.0
}

\author{
User's Manual \\ DAD_ESWL \\ Database-Assisted Design \& Equivalent Static Wind Load Procedures \\ for Mid- and High-Rise Structures
}

Last Updated by June 2018

Latest Version Available at www.nist.gov/wind

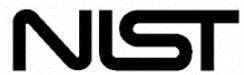

National Institute of Standards and Technology

Technology Administration, U.S. Department of Commerce 


\section{Disclaimers}

(1) Certain trade names or company products are specified in this document to specify adequately the procedure sued. Such identification does not imply recommendation or endorsement by NIST, nor does it imply that the product is the best available for the purpose.

(1-1) The "stand-alone" version of this software DAD_ESWL v1.0 requires installation of the MATLAB ${ }^{1}$ Component Runtime (MCR) Libraries provided by The MathWorks, Inc. The author's limited rights to the deployment of this program are limited by a license agreement between NIST and The MathWorks. The license agreement can be found at www.mathworks.com/license. The author, NIST, and The MathWorks and its licensors are excluded from all liability for damages or any obligation to provide remedial actions.

(1-2) The DAD_ESWL v1.0 includes the Open System for Earthquake Engineering Simulation (OpenSees) which is the open-source software framework for simulating dynamic responses of structural systems provided by The Regents of the University of California. The copyright agreement can be found at http://opensees.berkeley.edu/OpenSees/copyright.php.

The Regents grants permission, without fee and without a written license agreement, for (a) use, reproduction, modification, and distribution of this software and its documentation by educational, research, and non-profit entities for noncommercial purposes only; and (b) use, reproduction and modification of this software by other entities for internal purposes only.

Permission to incorporate this software into commercial products may be obtained by contacting the University of California [Bill Hoskins Office of Technology Licensing, 2150 Shattuck Avenue \#150 Berkeley, CA 94720-1620, (510) 643-7201].

This software program and documentation are copyrighted by The Regents of the University of California. The software program and documentation are supplied "as is", without any accompanying services from The Regents. The Regents does not warrant that the operation of the program will be uninterrupted or error-free. The end-user understands that the program was developed for research purposes and is advised not to rely exclusively on the program for any reason.

In no event shall the University of California be liable to any party for direct, indirect, special, incidental, or consequential damages, including lost profits, arising out of the use of this software and its documentation, even if the University of California has been advised of the possibility of such damage. The University of California specifically disclaims any warranties, including, but not limited to, the implied warranties of merchantability and fitness for a particular purpose. The software provided hereunder is on an "as is" basis, and the University of California has no obligations to provide maintenance, support, updates, enhancements, or modifications.

\footnotetext{
${ }^{1}$ MATLAB $^{\circledR}$. (c) 1994 - 2017 The MathWorks, Inc.
} 


\section{A1.1. Key features of DAD_ESWL software}

DAD_ESWL software is an integrated design tool providing time series of calculated wind-induced loads based on time series of aerodynamic pressures and inertial forces, as well as time series of member demand-to-capacity indexes, inter-story drift, and accelerations. This User's Manual is designed to assist the user of the software DAD_ESWL v1.0 for mid- and high-rise structures.

\section{A1.1.1 General features}

$\square$ Equivalent Static Wind Load (ESWL) approach

A simple approach to structural design, wherein the randomly fluctuating wind loads used in the DAD procedure are replaced by the ESWLs, is applied with this version of the software. DAD_ESWL provides two options for calculating the members' DCIs as the use of i) full time series of effective floor wind loads and ii) ESWLs.

\section{$\square$ Second-order effects}

Second-order elastic analysis that includes both $\mathrm{P}-\Delta$ (member chord rotation) and $\mathrm{P}-\delta$ (member curvature) effects is considered in the software by using a geometric stiffness method allowing the dynamic analysis to be performed without iterations (White and Hajjar 1991). For the frame analysis, the geometric stiffness matrix represents the stiffening and weakening effect by the tensile (positive) and compressive (negative) load in the structural members, respectively. The second-order problem can be formulated and solved as a linear system where the geometric stiffness matrix is subtracted from the elastic stiffness matrix. Since the lateral stiffness of the structural system is effectively reduced by the geometric stiffness matrix, the natural frequencies of vibration are lowered, and the modal shapes are slightly changed with respect to their counterparts determined without accounting for secondary effects.

\section{$\square$ Using OpenSees for calculation of structural properties}

DAD_ESWL can employ OpenSees software to calculate influence coefficients, dynamic properties (mode shapes and natural periods) and members' internal forces. Alternatively, those properties can be obtained by using finite element software. Details are provided in the following chapters.

$\square$ Calculating directly floor wind loads from measured pressures

The DAD_ESWL calculates floor aerodynamic load time series based on pressure time series. 


\section{A1.1.2 Special features for steel structures}

$\square$ Section details of steel members

Steel members consist of beams, columns, and diagonal bracings. Members are classified as compact, noncompact, or slender section according to the width-to-thickness ratios. DAD_ESWL v1.0 provides functions for determining member nominal capacities based on AISC Specifications for Structural Steel Buildings (ANSI/AISC 2010).

$\square$ Demand-to-capacity indexes (DCIs)

The demand-to-capacity index (DCI) is the left-hand side of the design interaction equation and is a measure of the degree to which structural members are designed adequately for strength. The final design for strength is achieved when the DCIs of members are less than and as close as possible to unity, to within specified serviceability constraints. The interaction formulas are updated for application to various types of beam-column joint, connection details of bracings, and shapes of section.

\section{A1.1.3 Special features for RC structures}

$\square$ Generation of biaxial interaction surfaces for $D C I^{P M}$ (also denoted by $B_{i j}{ }^{P M}$ in the MATLAB variable)

Once a reinforced concrete column or shear wall section is specified by the user, the DAD_ESWL v1.0 generates a biaxial interaction strength surface to determine the demand-to-capacity index for that member.

$\square$ DCIs for shear wall

The DCI can be determined for shear walls. For combined axial force and bending moment, the DCIs are calculated via biaxial interaction surfaces. The interaction formula for combined shear force and torsion is used as it was in the previous version (Yeo 2010). 


\section{A1.2. Download and installation}

DAD_ESWL can be accessed via the internet site www.nist.gov/wind. Under the heading [II. Wind Effects on Buildings], click the link [Wind Effects on Flexible Buildings]. This opens the main page [DAD_ESWL v1.0]. The stand-alone executable version of the DAD_ESWL v1.0 software is available in a bulleted list under the heading [Files available for download]. This executable file requires installation of MCRInstaller.exe which is also available on the main page. The bulleted list also includes the input files for the 60-story example described in the following chapter of this User's manual. The program opens a GUI (graphical user interface)-based window consisting of one initial page and five main pages: Bldg. modeling, Wind loads, Resp. surface, Wind effects, and Results \& Plots.

\section{A1.3. Basics of using the DAD_ESWL}

The DAD_ESWL has one initial page and five main pages (Bldg. modeling, Wind loads, Resp. surface, Wind effects, and Results \& Plots). In the initial page the user should select the type of structure (Steel or Reinforced concrete structure) and proceed to the main pages. The main pages are used to assign values to the variables used to model building and loads, and to perform the requisite response databases and the associated peak responses with specified mean recurrence intervals (MRIs). The 'Results \& Plots' page is used to show the results and the associated graphs.

Variable values can be loaded from a pre-assigned file by clicking 'Load inputs' button at the left bottom corner in any page. They also can be saved into a file by executing 'Save inputs' button at the right bottom corner in any page. The button 'Exit' next to the 'Save inputs' one enables to close the program by clicking it.

For running the example provided in the webpage of www.nist.gov/wind, it is recommended that a directory named ' $D A D \_E S W L$ ' be created on their local drive to save all downloaded files and directories. A recommended directory structure is shown in Fig. A1.1. The 'Building_data' directory contains the building model and wind loading input files, the 'OpenSees' directory contains the executable file of OpenSees (OpenSees.exe), the user's pre-defined Tcl file and other inputs related to use the OpenSees, the 'Aerodynamic_data' folder contains the time-series of the pressures for the 60-story example described in this manual, the 'Climatological_data_1', 'Climatological_data_2', and 'Climatological_data_3' directories contain simulated directional hurricane or nonhurricane wind speed files, and the 'Output' directory stores the analysis results after running the software, respectively. Note that these recommendations are not mandatory for users.

Figure A1.1. Recommended directory structure

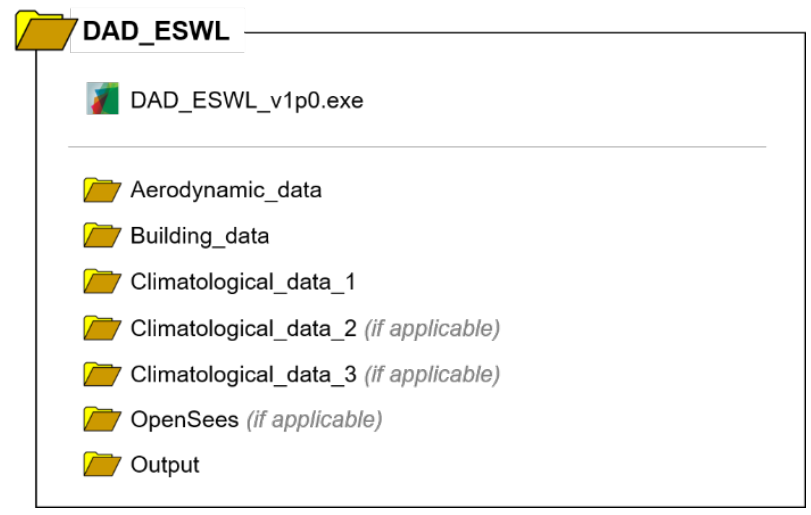




\section{A1.4. Inputs for running DAD_ESWL}

The DAD_ESWL requires as input information including details on the structural members, time series of aerodynamic pressures on the external surfaces, and wind climatological data at the location of the structure, as follows:

$\square$ Details on structural members and whole structure

- Dimensions of members

- Shapes of sections (W or HSS, in case of steel structure)

- Reinforcement details (in case of RC structure)

- Number of stories

- Heights of floors

$\square$ Structural static and dynamic properties

- Number of modes and modal damping ratios

- Mass and mass moment of inertia with respect to mass center for each floor

In the case that the user chooses to input data from arbitrary FE software,

- Influence coefficients, defined as the internal forces that occur in each section of a given member due to a unit load applied to the center of mass of a given floor

- Internal forces on members due to dead, super-imposed dead, and live loads

- Mode shapes

- Modal periods

If the user chooses to use OpenSees for calculations,

- User's pre-defined Tcl script file which must include ModelBuilder object only. In other words, this Tcl script should exclusively have nodal connectivity, types of element, geometrical information of each element, and material properties. The other objects, such as Analysis (including loads and load cases) and Recorder, will be included automatically in the DAD_ESWL v1.0

- Connectivity data (labels of nodes and elements and their coordinates)

- Gravity loads data (dead, super-imposed dead, and live load values associated with each label of elements)

$\square$ Wind pressure data or Wind loads

- Time-varying pressures (i.e., $C_{p}$ ) or wind loads applied at the mass center of each floor, obtained from wind tunnel tests using a rigid model or CFD analysis

$\square$ Wind climate at or near the building site

- Databases of directional wind speeds for each type of wind occurring at the site (e.g., hurricane, thunderstorm, and synoptic wind)

- Ratio of 3s-averaged wind speeds at $10 \mathrm{~m}$ above in open terrain exposure to their mean hourly wind speed at the top of the building in the terrain exposure at that site. 


\section{A1.5. Outputs from running DAD_ESWL}

- Peak demand to capacity indexes for all members, for specified Mean Recurrence Intervals (MRIs)

- Peak inter-story drift ratios calculated along selected column lines, for specified MRIs

- Peak resultant accelerations calculated along selected column lines, corresponding to specified MRIs

\section{A1.6. Details on use of the software}

This section illustrates in detail how to input the requisite information for steel and for reinforced concrete buildings. General description of input variables and their associated formats are provided in the subsequent pages.

\section{A1.6.1 Example of a 3D 60-story steel building}

Consider a 60-story steel building (known as the CAARC building, Melbourne 1980), with floors assumed to be rigid diaphragms (Fig. A1.2). The building is $45.72 \mathrm{~m}$ in width, 30.48 $\mathrm{m}$ in depth, and $182.88 \mathrm{~m}$ in height, and has 6 by 4 bays. It has an outrigger and belt truss system located on $20^{\text {th }}, 21^{\text {st }}, 40^{\text {th }}, 41^{\text {st }}$, and $60^{\text {th }}$ story, and consists of 2100 columns, 3480 beams, and 2560 diagonal bracings.

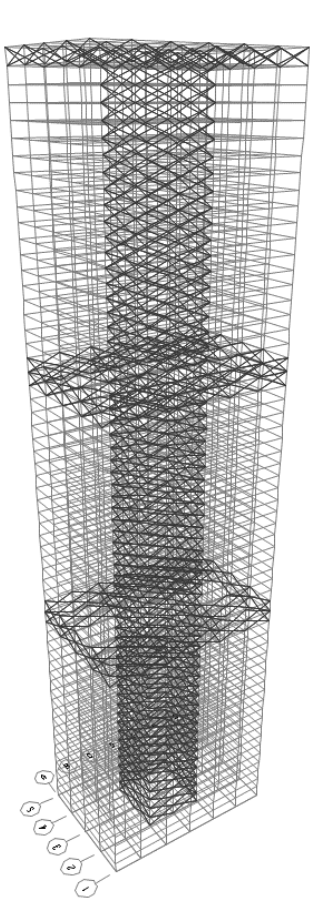

(a) 3D view

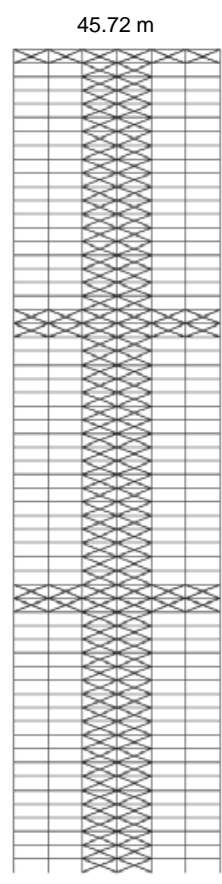

(b) Front view

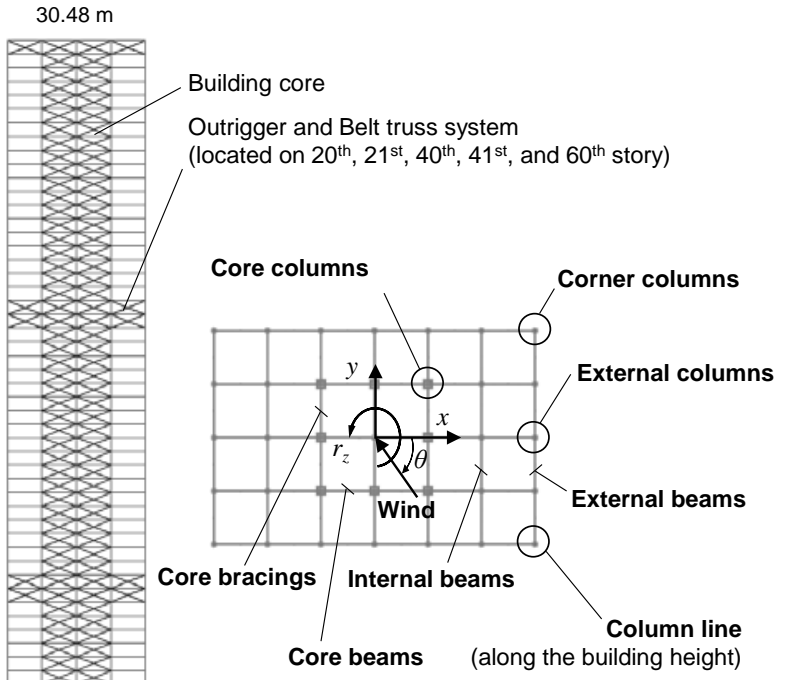

(d) Plan view and selected members and column line ( $\theta=$ wind direction)

Figure A1.2. Schematic views of a 60-story steel building 


\section{A1.6.2 Example of a 3D 60-story reinforced concrete building}

Consider a 60-story reinforced concrete building (known as the CAARC building, same as the steel building above, Fig. A1.3). The building is $45.72 \mathrm{~m}$ in width, $30.48 \mathrm{~m}$ in depth, and $182.88 \mathrm{~m}$ in height, and has 7 by 5 bays. It has a shear wall core system located on entire stories, and consists of 2400 columns, 4560 beams.

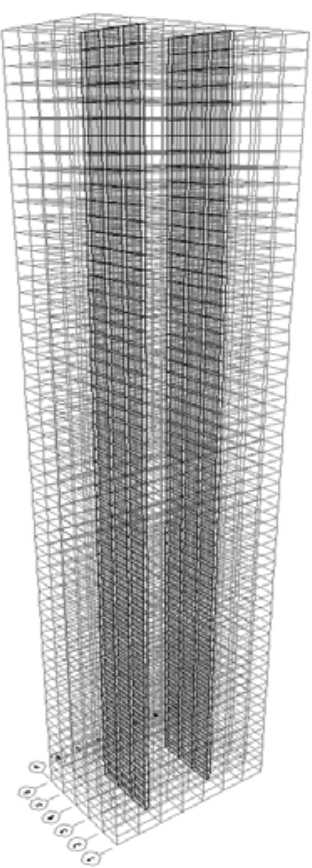

(a) 3D view

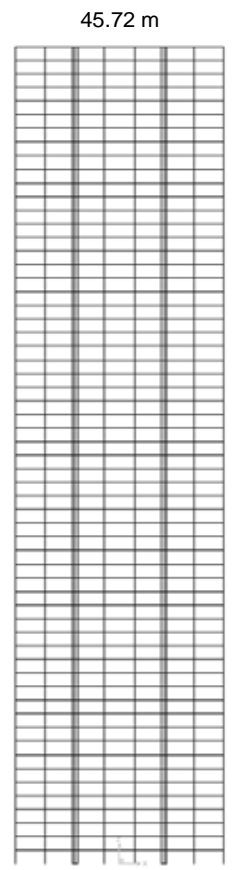

(b) Front view
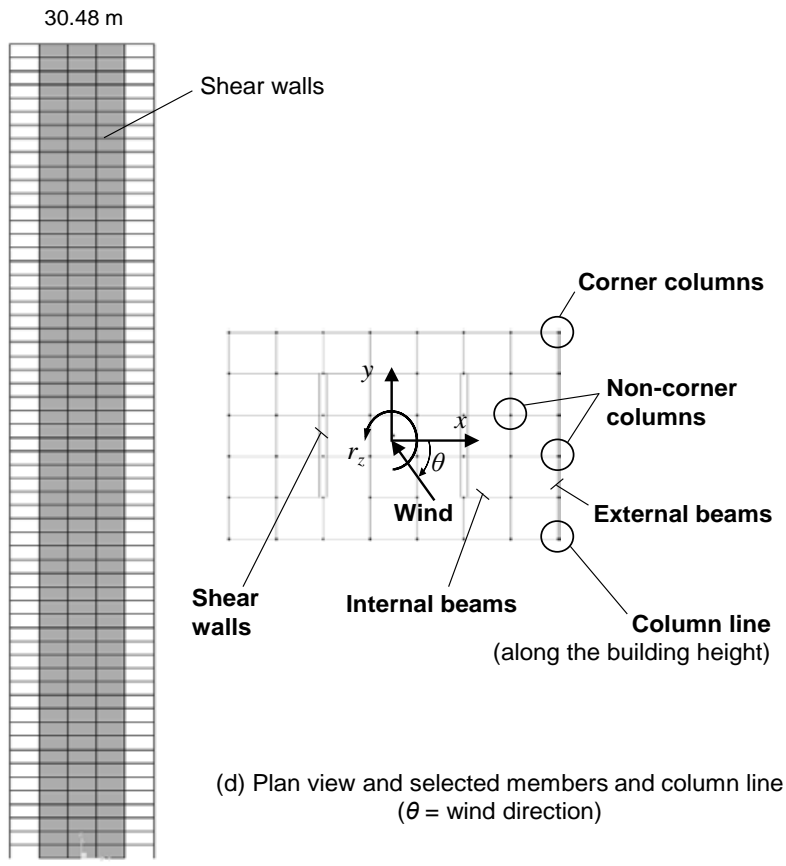

(d) Plan view and selected members and column line $(\theta=$ wind direction $)$

Figure A1.3. Schematic views of a 60-story reinforced concrete building 


\section{A1.6.3 Graphical user interface}

\section{Getting started}

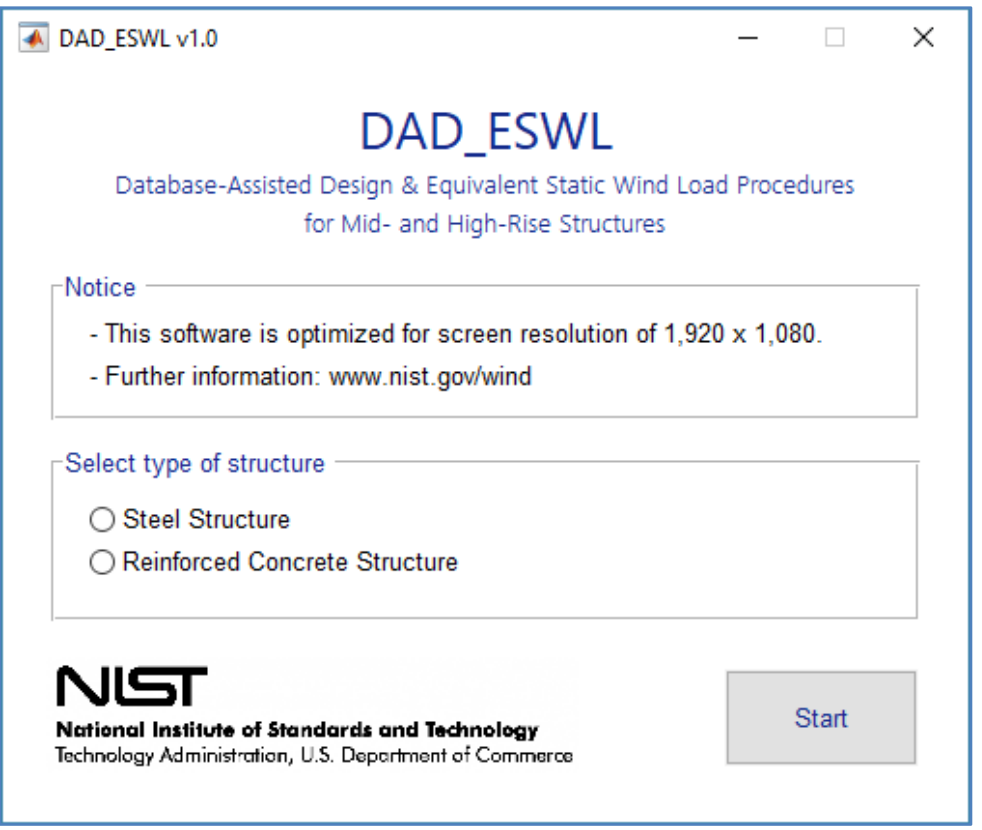

\section{Select type of structure}

Launching the DAD_ESWL V1.0 opens the graphical user interface shown above, and prompts the user to select the type of structure.

After clicking the Start button the user proceeds to the next step of this software. 


\section{$\square$ Building modeling}

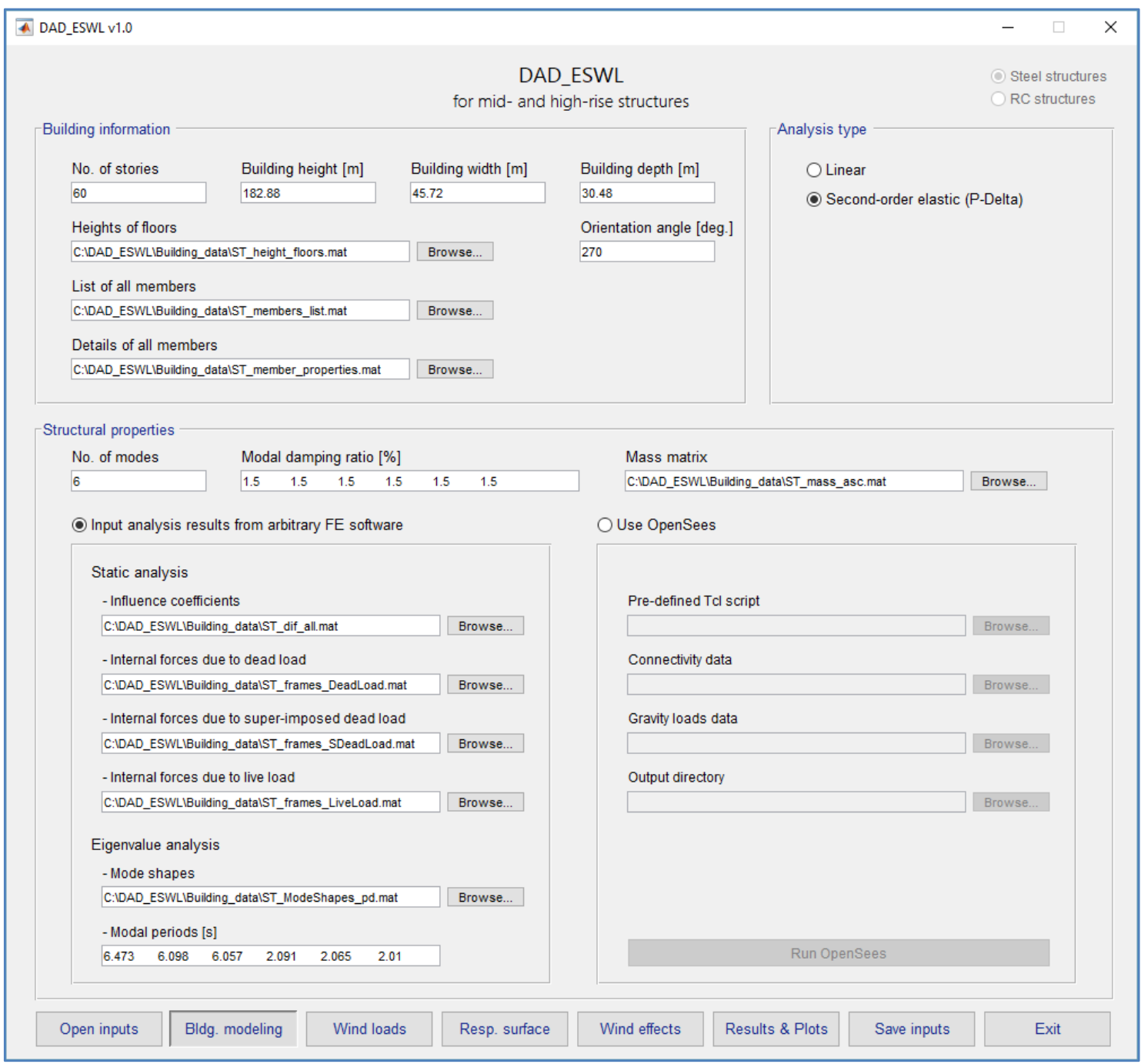

\section{Building information}

\begin{tabular}{|c|c|}
\hline No. of stories & $\begin{array}{l}: \text { Number of stories (or floors) } \\
\text { - Nfloors }=60\end{array}$ \\
\hline Building height [m] & $\begin{array}{l}\text { : Building height in } \mathrm{m} \\
\mathbf{H} \mathbf{H} \_ \text {bldg }=182.88\end{array}$ \\
\hline Building width [m] & $\begin{array}{l}\text { : Building width in } \mathrm{m} \\
\text { B_bldg }=45.72\end{array}$ \\
\hline Building depth [m] & $\begin{array}{l}\text { : Building depth in } \mathrm{m} \\
\mathbf{L} \mathbf{L} \text { bldg }=30.48\end{array}$ \\
\hline
\end{tabular}




\begin{tabular}{|c|c|}
\hline Orientation angle [deg.] & $\begin{array}{l}\text { : Orientation angle }\left(\alpha_{0}\right) \text { of a building in the clockwise direction from the North } \\
\text { to the } x \text {-axis of the building }\left(0^{\circ} \leq \alpha_{0}<360^{\circ}\right) \\
\text { Ang_orn = } 270 \text { (deg.) } \\
\text { Note) When the terrain exposure does not change around a building, the } \\
\text { orientation angle of the building can be changed. However, for direction- } \\
\text { dependent terrain conditions, various orientation angles can be considered } \\
\text { only when aerodynamic data from wind tunnel tests account for terrain } \\
\text { exposure corresponding to the various building orientations of interest. }\end{array}$ \\
\hline Heights of floors & $\begin{array}{l}\text { : Load the MATLAB file containing the heights of all floors } \\
\text { The variable is named } \mathbf{H} \text { floor and can be saved in a mat file with an arbitrary } \\
\text { name. This variable is a row vector containing the heights of all floors and } \\
\text { roof except for the ground floor. For example, the heights of floors in a } 60- \\
\text { story building are }[3.0486 .096 \ldots 182.88] \text {. } \\
\text { H_floor }(1 \times \text { No. of stories })= \\
\qquad\left[\text { height at } 1^{\text {st }} \text { floor height at } 2^{\text {nd }} \text { floor } \cdots \text { height at roof }\right] \\
\text { The unit is } \mathrm{m} \text {. }\end{array}$ \\
\hline List of all members & $\begin{array}{l}\text { : Load the MATLAB file containing the list of members of the structure } \\
\text { The variable is named mem_list and can be saved in a mat file with an } \\
\text { arbitrary name. The variable is a matrix with three rows and number of } \\
\text { columns that make up the structure. The first row contains the member } \\
\text { numbers. The second row has types of members using string characters (i.e., } \\
\text { "C", "B", "W", or "X" that represents column, beam, shear wall, and diagonal } \\
\text { bracing, respectively.) The third row contains the identifier of the member } \\
\text { defined in the second row (e.g., } 1 \text { for "C" represents column 1, and } 2 \text { for "B" } \\
\text { beam 2.) } \\
\text { mem_list ( } 3 \times \text { No. of members) }= \\
\qquad\left[\begin{array}{cccccc}1 & 2 & \cdots & n & \cdots & \text { No. of members } \\
\text { 'C' } & \text { 'C' } & \cdots & \text { 'B' } & \cdots \\
1 & 1 & \cdots & 1 & \cdots\end{array}\right] \\
\text { contains } 12 \text { types of columns, } 1 \text { type of beams, and } 3 \text { types of diagonal } \\
\text { bracings, respectively. }\end{array}$ \\
\hline
\end{tabular}




\begin{tabular}{|c|c|}
\hline Details of all members & : Load the MATLAB file containing the section properties \\
\hline i) Steel structures & 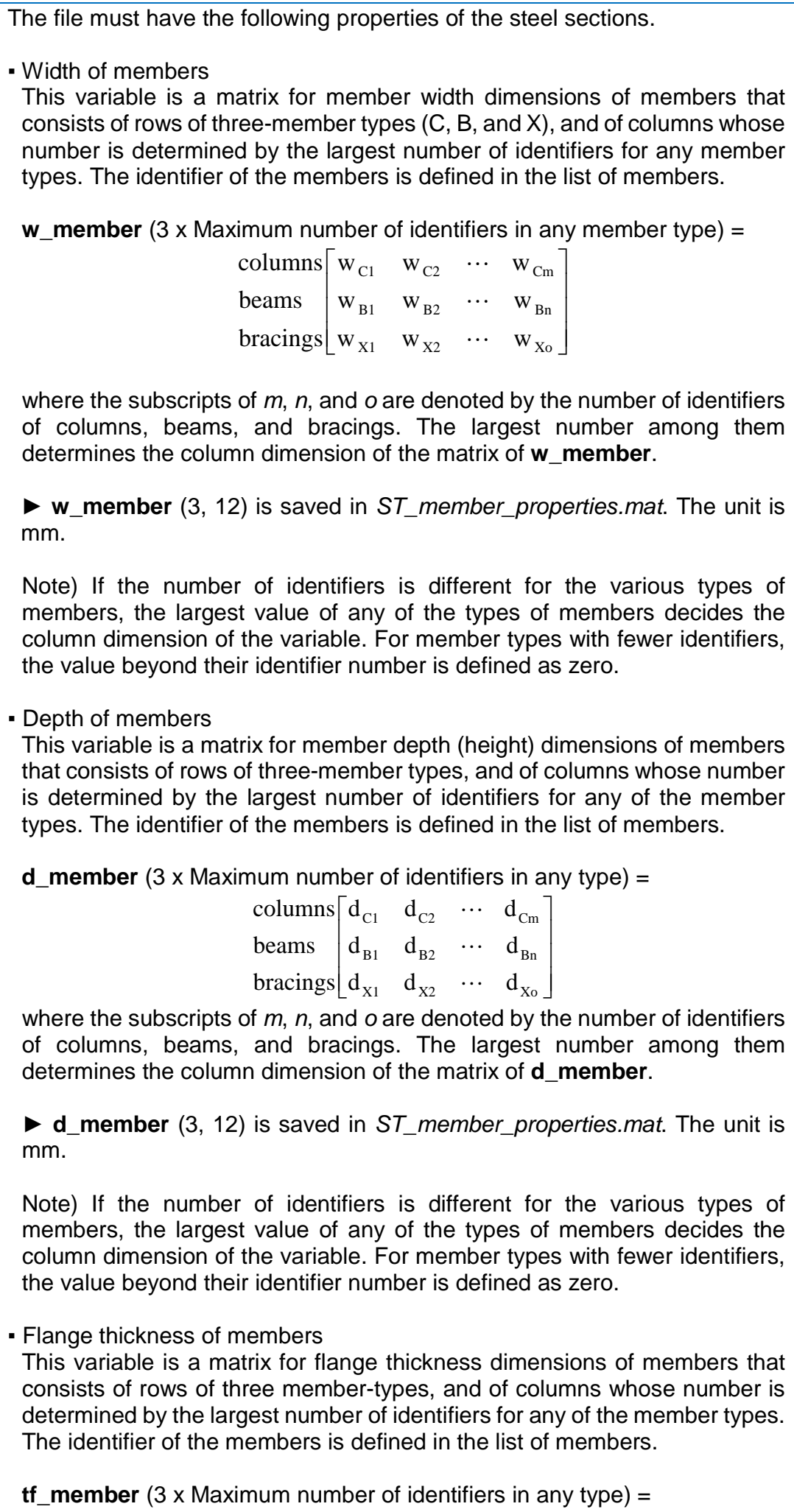 \\
\hline
\end{tabular}




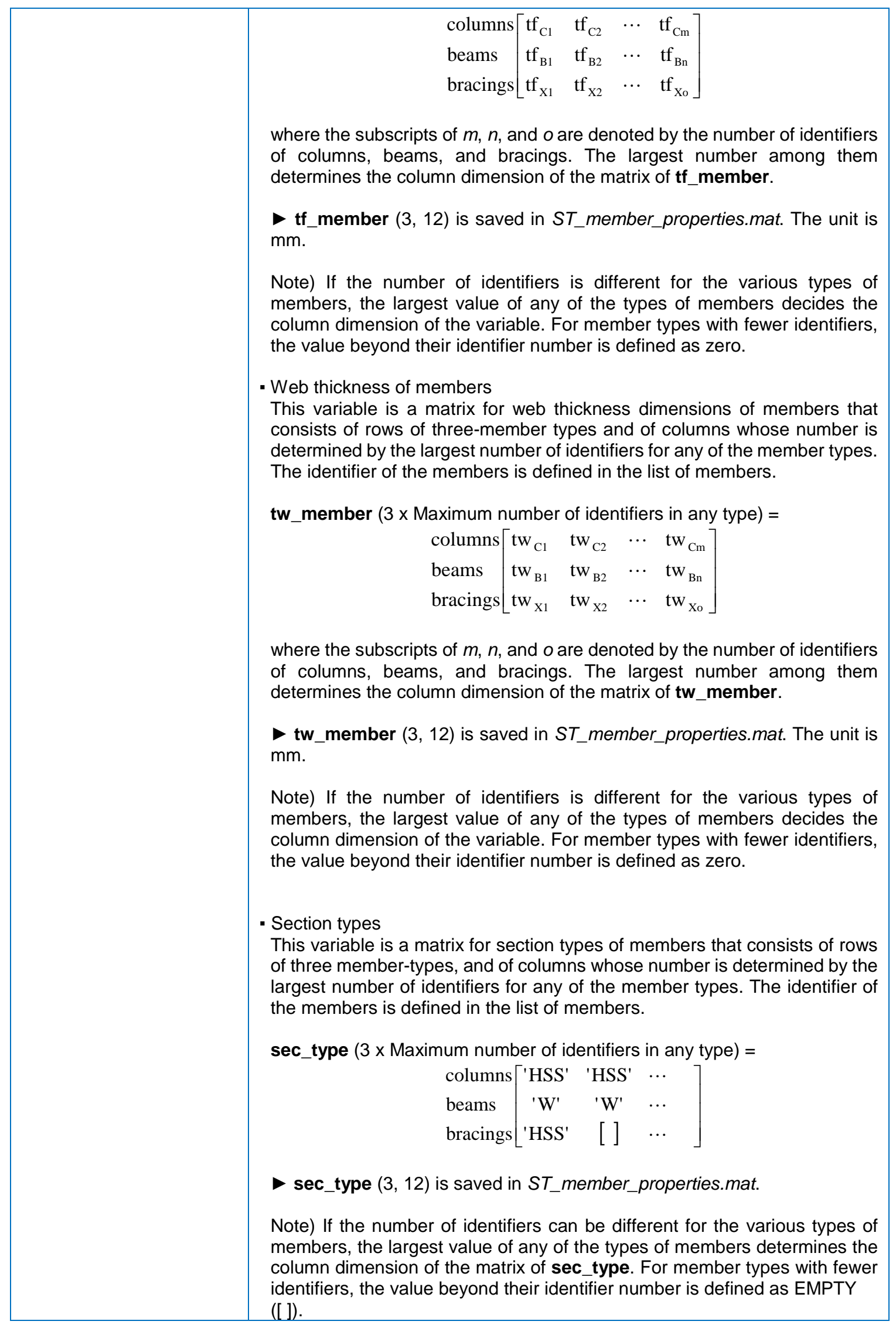


- Assuming sections as compact

This variable is a matrix for section classifications of members that consists of rows of three member-types, and of columns whose number is determined by the largest number of identifiers for any of the member types. The identifier of the members is defined in the list of members. Each cell has character values of 'auto' or 'compact'. If the user applies the 'auto' identifier for the section of interest, the software will automatically decide whether that section is compact, non-compact, or slender. If the 'compact' identifier is applied, on the other way, that section will be assumed to have compact flange and web regardless of its sectional dimensions.

sec_compact $(3 \times$ Maximum number of identifiers in any type $)=$

$$
\begin{aligned}
& \text { columns } \\
& \text { beams } \\
& \text { bracings }
\end{aligned}\left[\begin{array}{ccc}
\text { 'compact' } & \text { 'compact' } & \ldots \\
\text { 'auto' } & \text { 'auto' } & \ldots \\
\text { 'auto' } & {[]} & \ldots
\end{array}\right]
$$

sec_compact $(3,12)$ is saved in ST_member_properties.mat.

Note: If the number of identifiers can be different for the various types of members, the largest value of any of the types of members determines the column dimension of the matrix of sec_compact. For member types with fewer identifiers, the value beyond their identifier number is defined as EMPTY ([ ]).

- Miscellaneous sectional variables stored in this file The following variables have the same structure with the variables stated above, and are saved in ST_member_properties.mat.

Area of members $\left(\mathrm{mm}^{2}\right)$ :

- A_member $(3,12)$

Torsional constant of members $\left(\mathrm{mm}^{4}\right)$ :

- J_member $(3,12)$

Moment of inertia for bending about the local 3-axis of members $\left(\mathrm{mm}^{4}\right)$ :

133_member $(3,12)$

Moment of inertia for bending about the local 2-axis of members $\left(\mathrm{mm}^{4}\right)$ :

- 122_member $(3,12)$

Shear area in the local 3-axis of members $\left(\mathrm{mm}^{2}\right)$ :

- A_v3_member $(3,12)$

Shear area in the local 2-axis of members $\left(\mathrm{mm}^{2}\right)$ :

- A_v2_member $(3,12)$

Section modulus for bending about the local 3-axis of members $\left(\mathrm{mm}^{3}\right)$ :

- S33_member $(3,12)$

Section modulus for bending about the local 2-axis of members $\left(\mathrm{mm}^{3}\right)$ :

- S22_member $(3,12)$

Radius of gyration about the local 3-axis of members ( $\mathrm{mm})$ :

- R33_member $(3,12)$

Radius of gyration about the local 2-axis of members ( $\mathrm{mm})$ :

- R22_member $(3,12)$

Plastic modulus for bending about the local 3-axis of members $\left(\mathrm{mm}^{3}\right)$ :

- Z33_member $(3,12)$ 


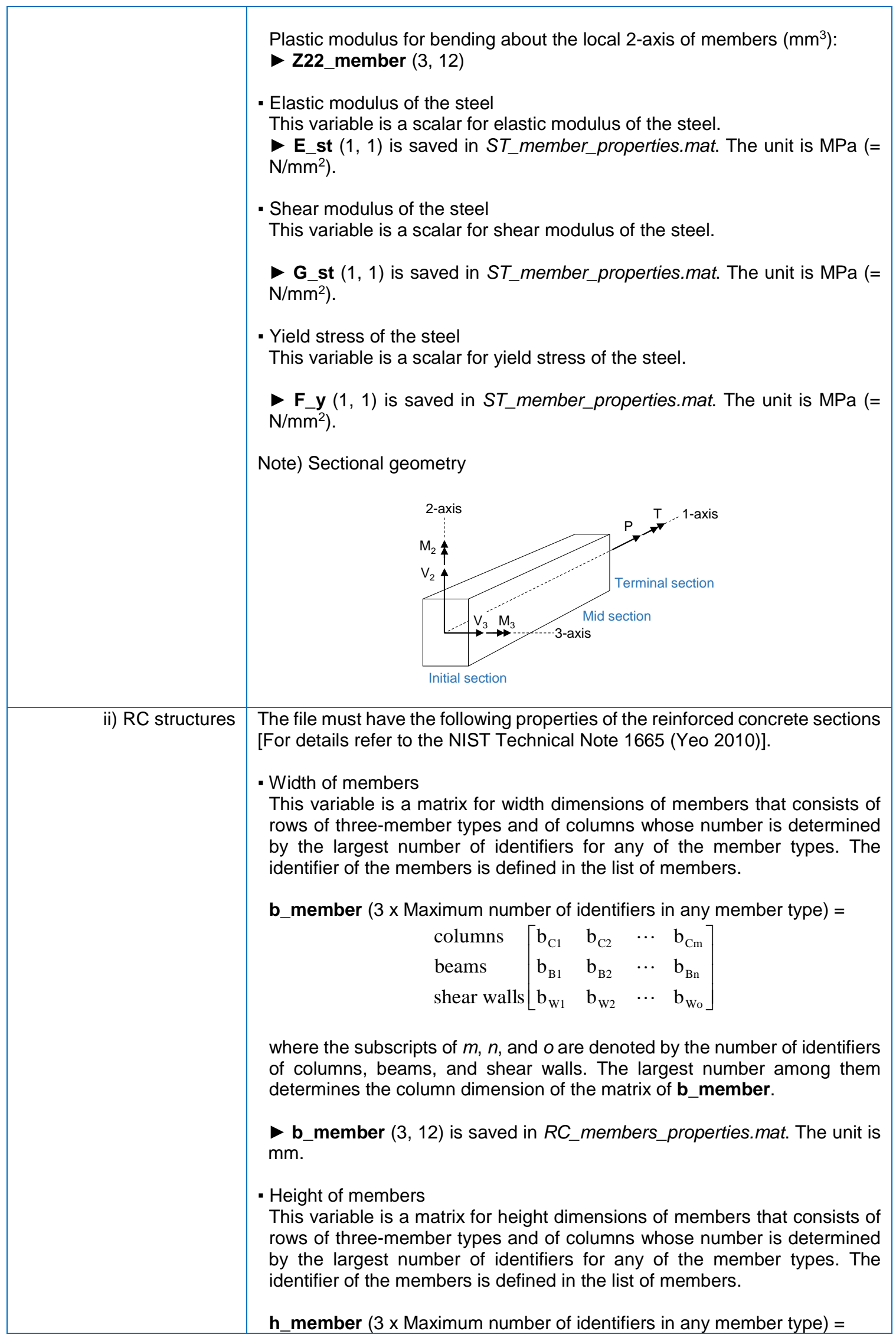




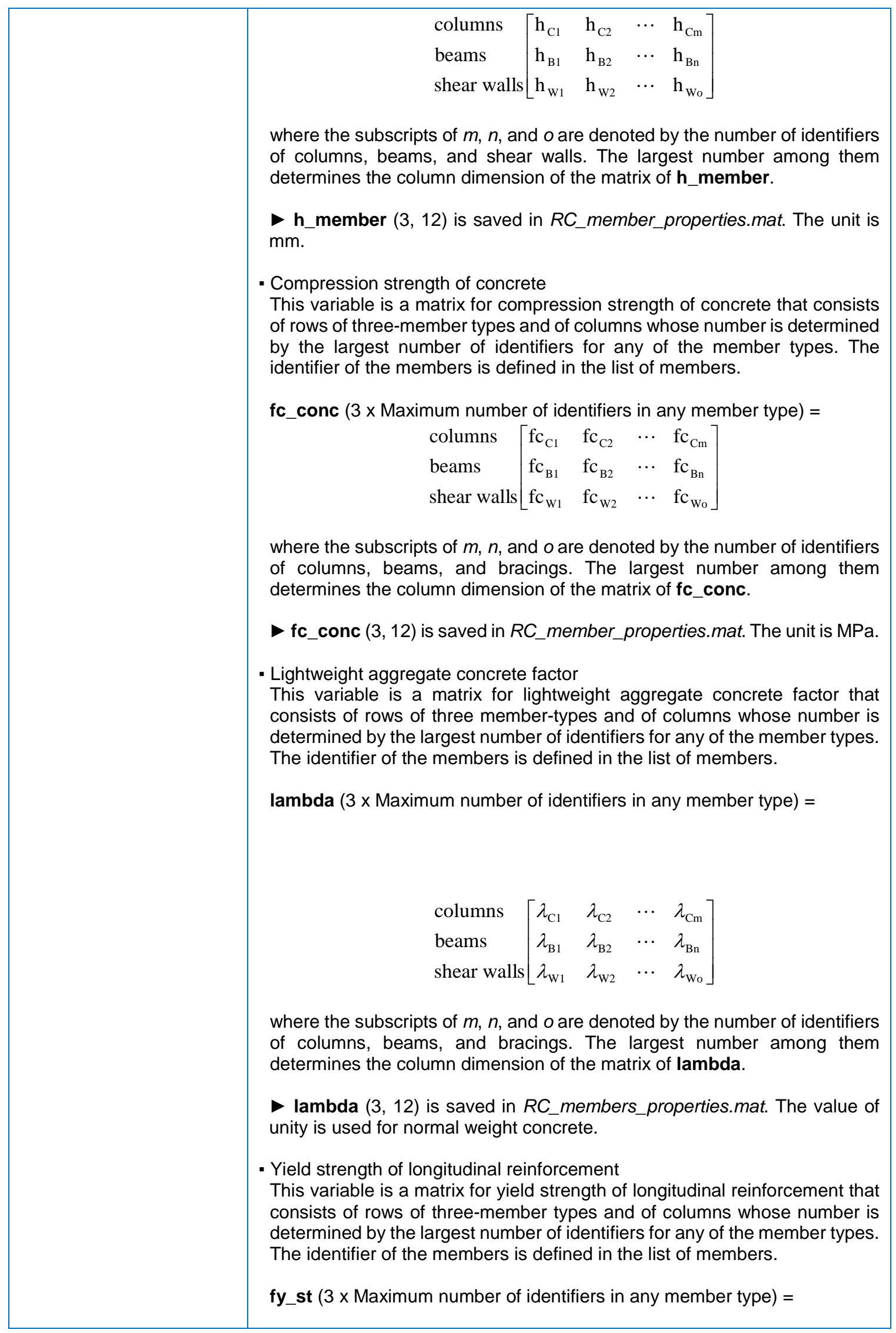




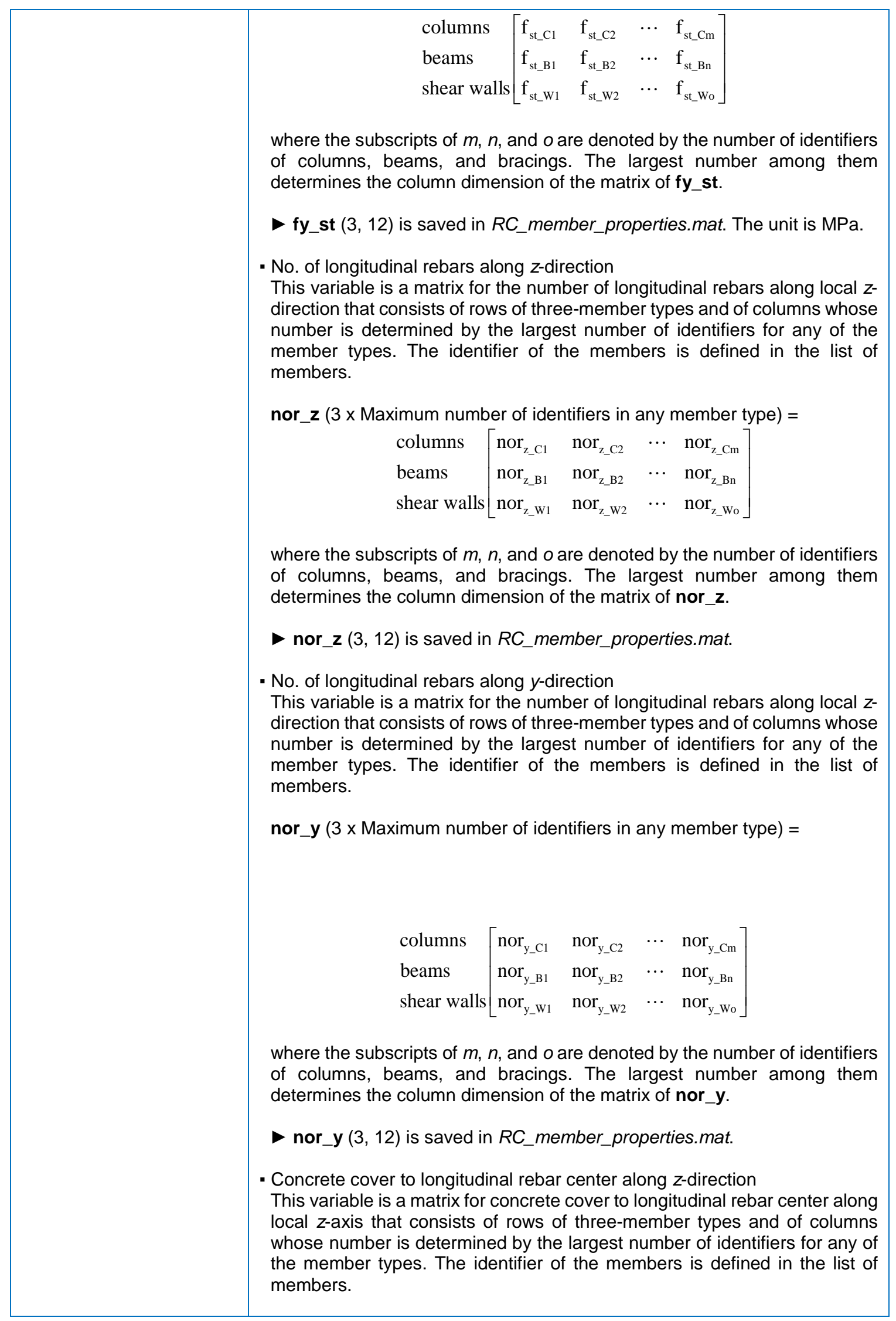




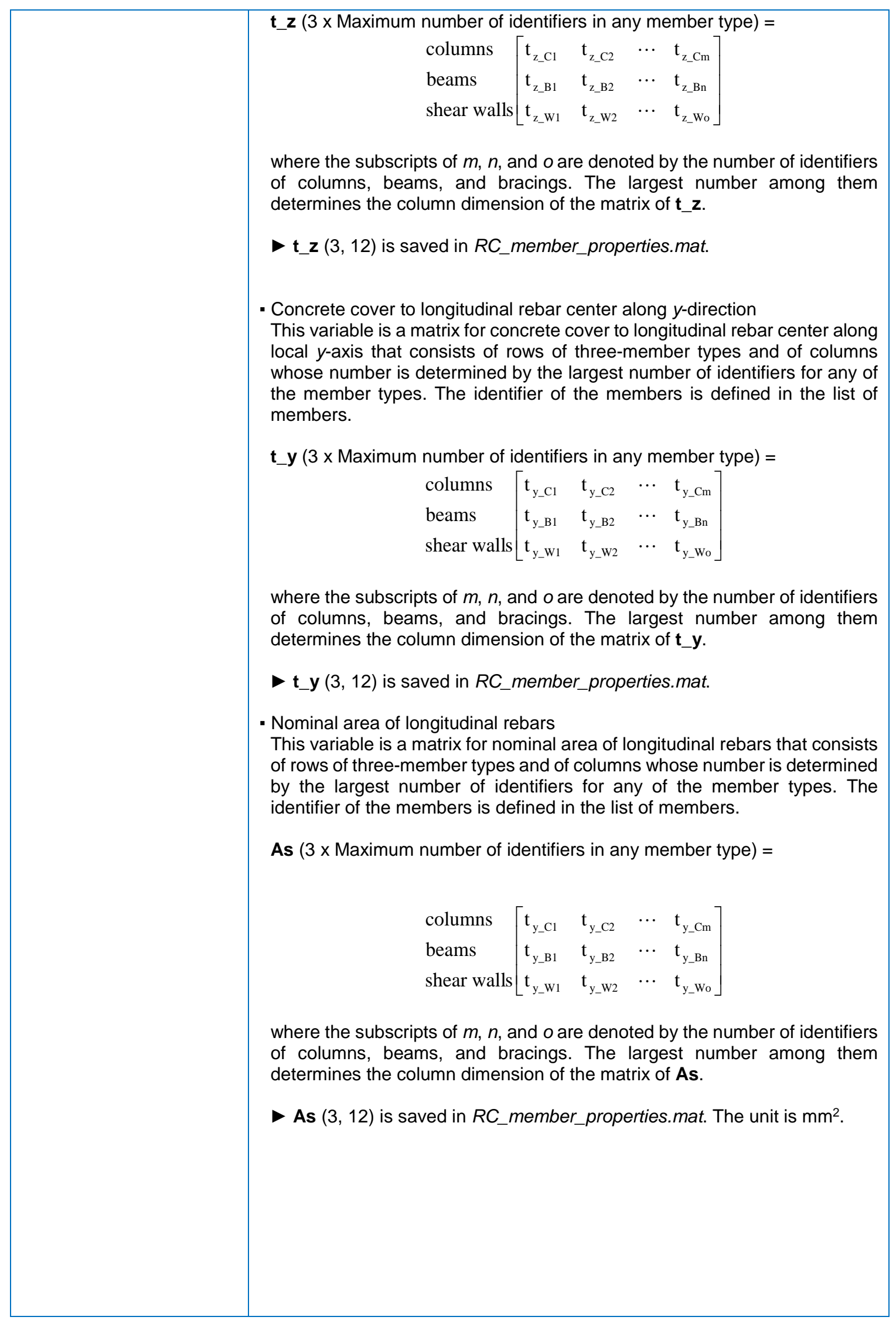




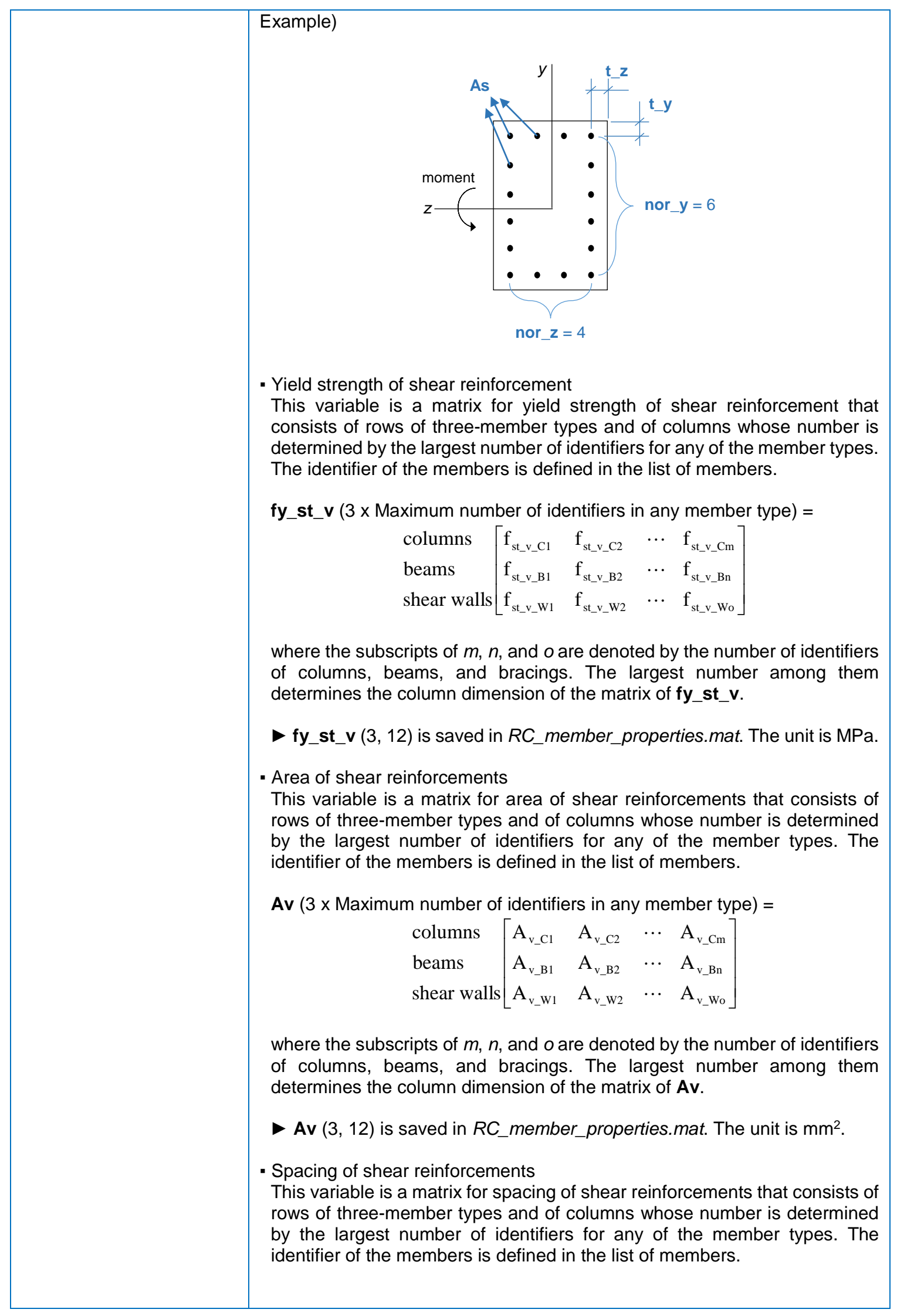




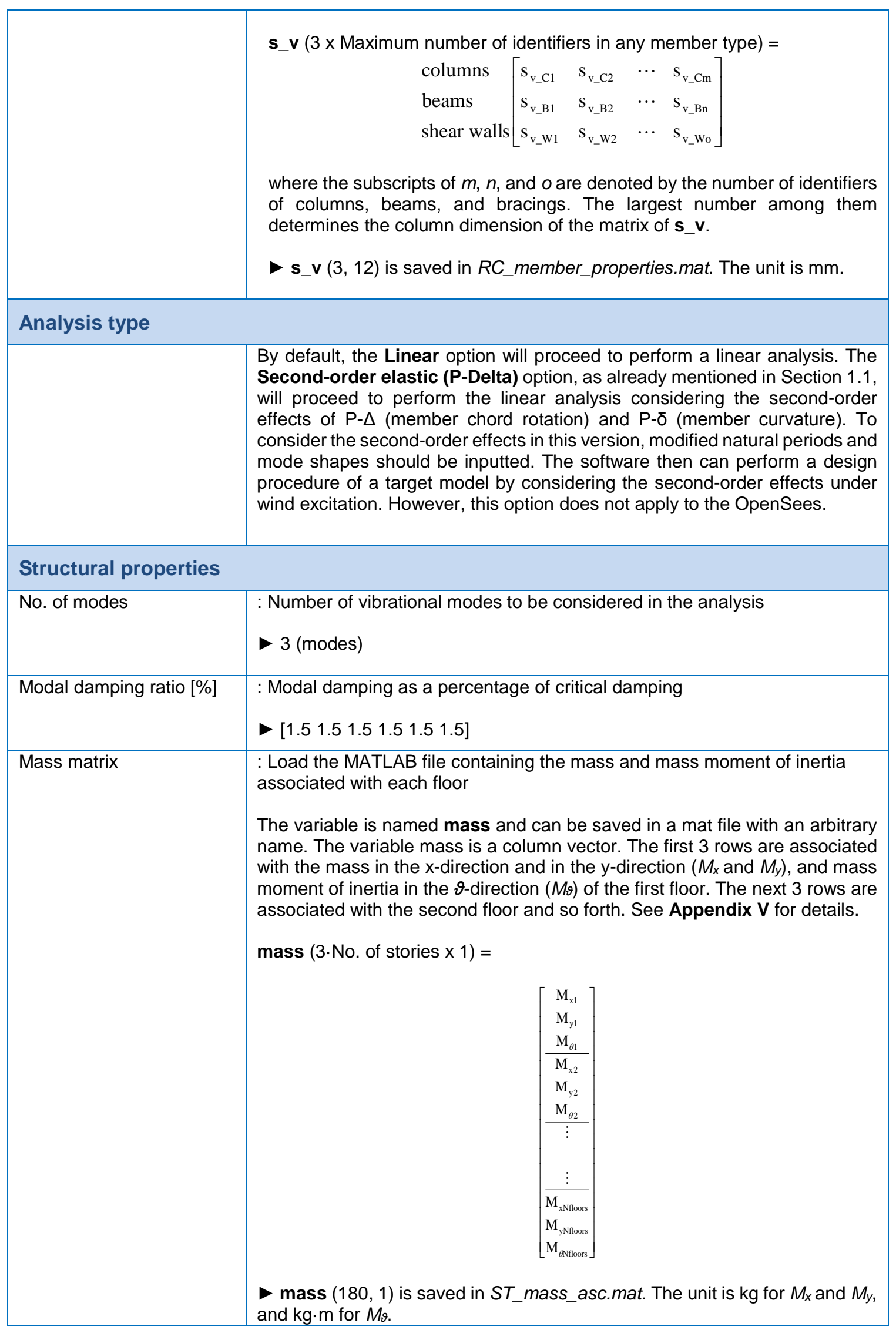




\begin{tabular}{|c|c|}
\hline \multicolumn{2}{|c|}{ Input analysis results from arbitrary FE software } \\
\hline Influence coefficient & 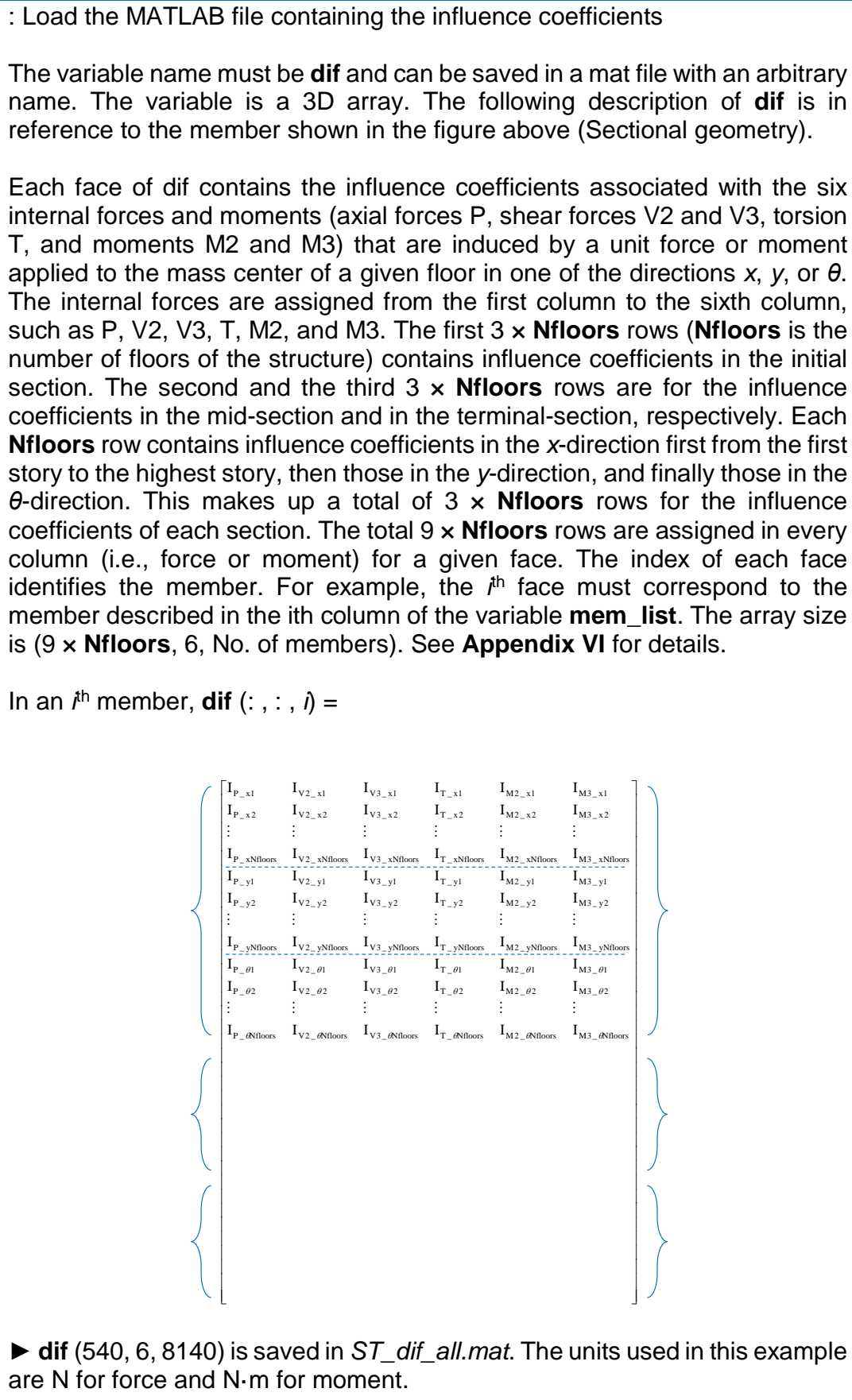 \\
\hline $\begin{array}{l}\text { Internal forces due to } \\
\text { dead load }\end{array}$ & $\begin{array}{l}\text { : Load the MATLAB file containing the contribution of the dead loads } \\
\text { The variable is named frames_DL and can be saved in a mat file with an } \\
\text { arbitrary name. The variable frames_DL is a matrix with the first column of } \\
\text { member IDs that coincide with the first row of mem_list. Each row contains } \\
\text { the internal forces due to dead loads occurring in the initial, mid, and terminal } \\
\text { sections of the member identified in the first column of the row. All } 6 \text { internal } \\
\text { forces and moments (P, V2, V3, T, M2, and M3) are assigned from columns } \\
2 \text { to } 7 \text { for the initial section, from columns } 8 \text { to } 13 \text { for the mid-section, and from } \\
\text { columns } 14 \text { to } 19 \text { for the terminal section. }\end{array}$ \\
\hline
\end{tabular}




\begin{tabular}{|c|c|}
\hline & $\begin{array}{l}\text { frames_DL (No. of members }(=\mathbf{N m}) \times 19) \\
\left.\qquad \begin{array}{llllllll}1 & \mathrm{P}_{\mathrm{D} 1} & \mathrm{~V}_{\mathrm{D} 1} & \mathrm{~V}_{\mathrm{D} 1} & \mathrm{~T}_{\mathrm{D} 1} & \mathrm{M} 2_{\mathrm{D} 1} & \mathrm{M} 3_{\mathrm{D} 1} \\
2 & \mathrm{P}_{\mathrm{D} 2} & \mathrm{~V}_{\mathrm{D} 2} & \mathrm{~V} 3_{\mathrm{D} 2} & \mathrm{~T}_{\mathrm{D} 2} & \mathrm{M} 2_{\mathrm{D} 2} & \mathrm{M}_{\mathrm{D} 2} \\
\vdots & \vdots & \vdots & \vdots & \vdots & \vdots & \vdots \\
\mathrm{Nm} & \mathrm{P}_{\mathrm{DNm}} & \mathrm{V}_{\mathrm{DNm}} & \mathrm{V}_{\mathrm{DNm}} & \mathrm{T}_{\mathrm{DNm}} & \mathrm{M} 2_{\mathrm{DNm}} & \mathrm{M}_{\mathrm{DNm}}\end{array}\right] \\
\text { frames_DL (8140, 19) is saved in frames_DeadLoad.mat. The units are N } \\
\text { for forces and } \mathrm{N} \cdot \mathrm{m} \text { for moments. } \\
\text { Note) The forces and moments of frames_DL are due to unfactored dead } \\
\text { loads. They can be calculated using a finite element analysis software, or the } \\
\text { OpenSees provided in this software. For details see the next section Use } \\
\text { OpenSees for calculation. }\end{array}$ \\
\hline $\begin{array}{l}\text { Internal forces due to } \\
\text { super-imposed dead load }\end{array}$ & 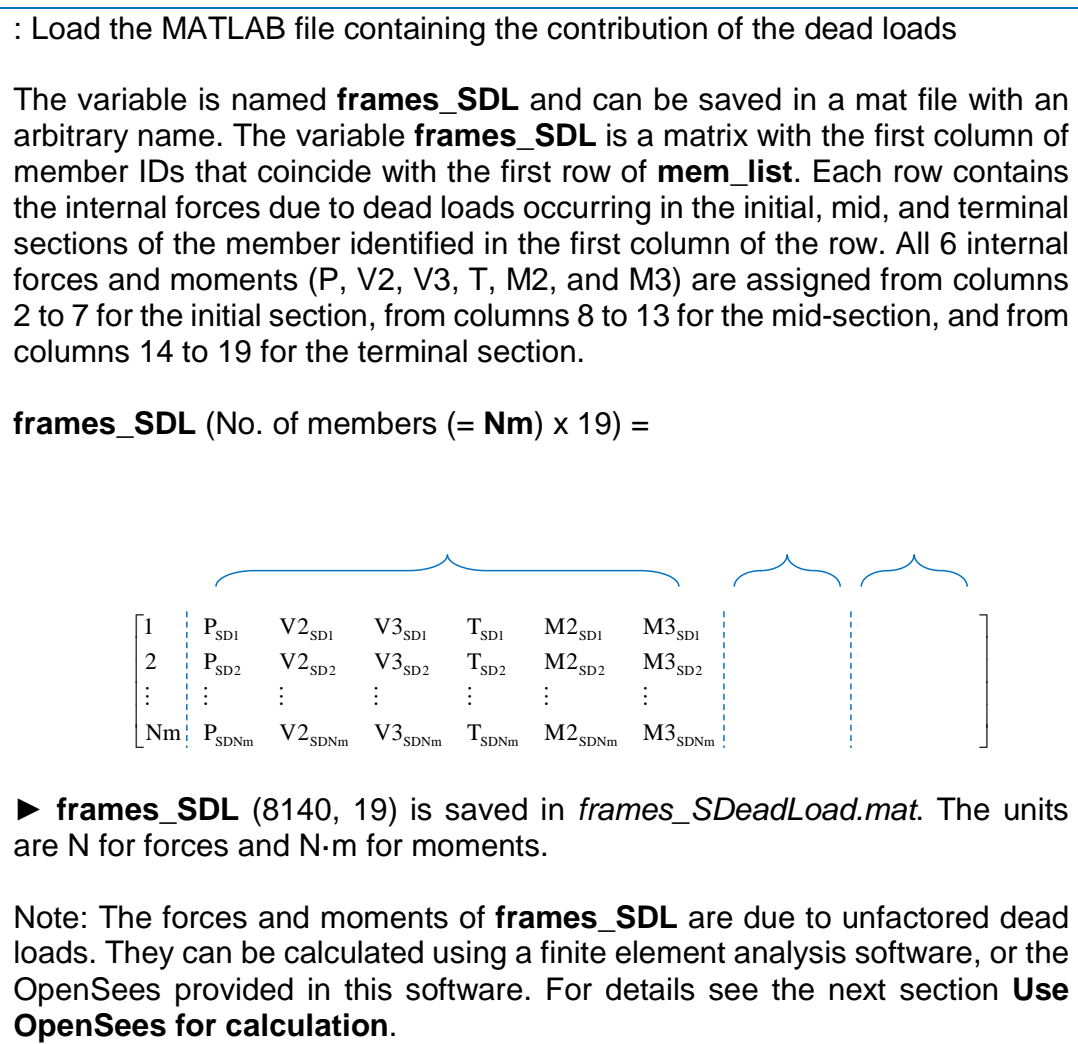 \\
\hline $\begin{array}{l}\text { Internal forces due to live } \\
\text { load }\end{array}$ & $\begin{array}{l}\text { : Load the MATLAB file containing the contribution of the dead loads } \\
\text { The variable is named frames_LL and can be saved in a mat file with an } \\
\text { arbitrary name. The variable frames_LL is a matrix with the first column of } \\
\text { member IDs that coincide with the first row of mem_list. Each row contains } \\
\text { the internal forces due to dead loads occurring in the initial, mid, and terminal } \\
\text { sections of the member identified in the first column of the row. All } 6 \text { internal } \\
\text { forces and moments }(P, V 2, V 3, T, M 2 \text {, and M3) are assigned from columns } \\
2 \text { to } 7 \text { for the initial section, from columns } 8 \text { to } 13 \text { for the mid-section, and from } \\
\text { columns } 14 \text { to } 19 \text { for the terminal section. } \\
\text { frames_LL (No. of members }(=\mathrm{Nm}) \times 19)=\end{array}$ \\
\hline
\end{tabular}




\begin{tabular}{|c|c|}
\hline \multicolumn{2}{|c|}{ Use OpenSees for calculation } \\
\hline Pre-defined Tcl script & 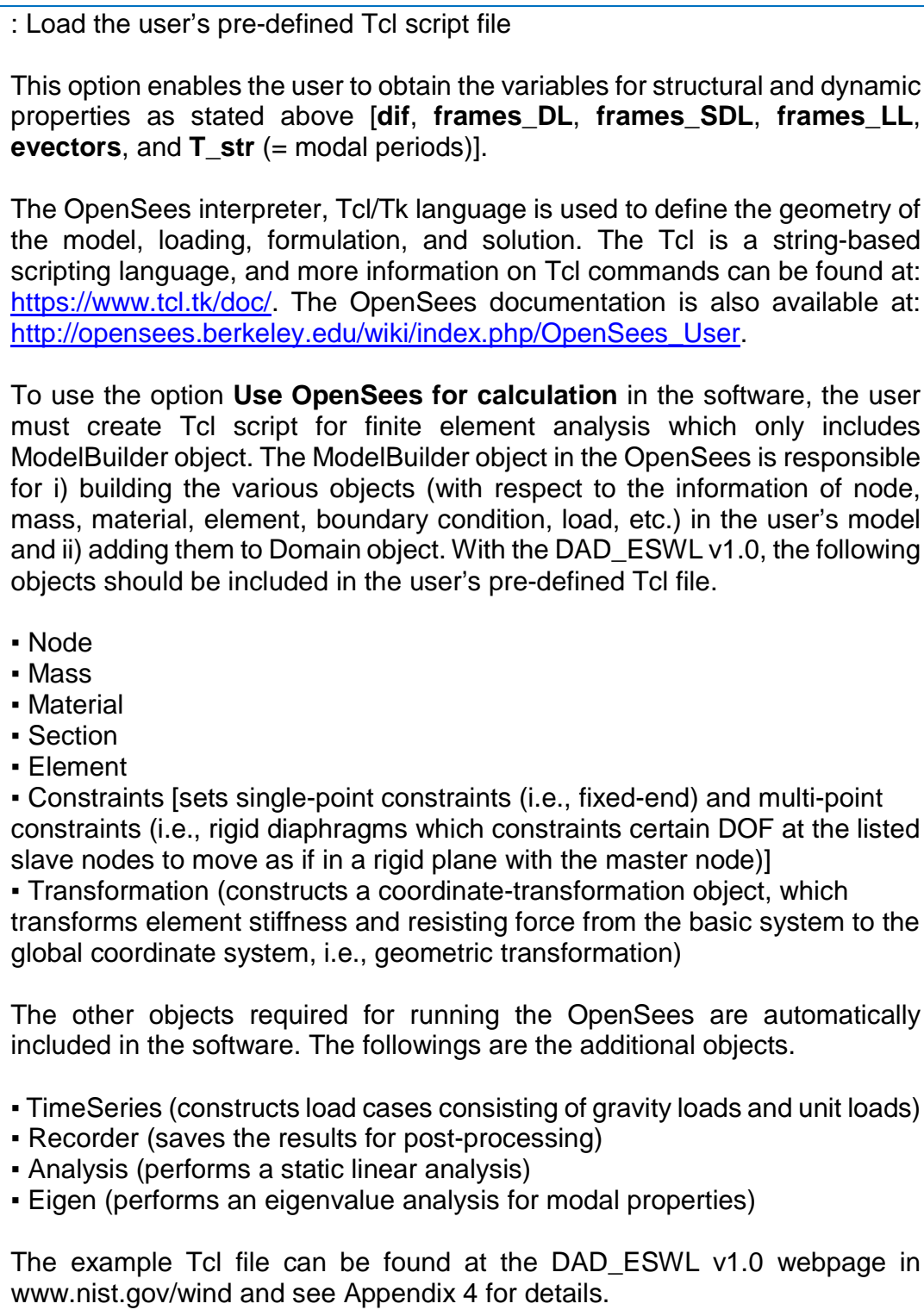 \\
\hline Connectivity data & $\begin{array}{l}\text { : Load the MATLAB file containing the connectivity data for the OpenSees } \\
\text { model } \\
\text { The variable is named Coord and can be saved in a mat file with an arbitrary } \\
\text { name. The variable is a structure array with the specified field and values. } \\
\text { The following fields must be included in the structure variable Coord. } \\
\text { - Ncoord (No. of nodes } x 4 \text { ) } \\
\qquad\left[\begin{array}{llll}1 & \mathrm{x}_{1} & \mathrm{y}_{1} \\
2 & \mathrm{x}_{2} & \mathrm{y}_{2} & \mathrm{z}_{2} \\
\vdots & \vdots & \vdots & \vdots \\
\text { No. of nodes } & \mathrm{x}_{\mathrm{n}} & \mathrm{y}_{\mathrm{n}} & \mathrm{z}_{\mathrm{n}}\end{array}\right] \\
\text { where the subscript of the coordinates is the node label. }\end{array}$ \\
\hline
\end{tabular}




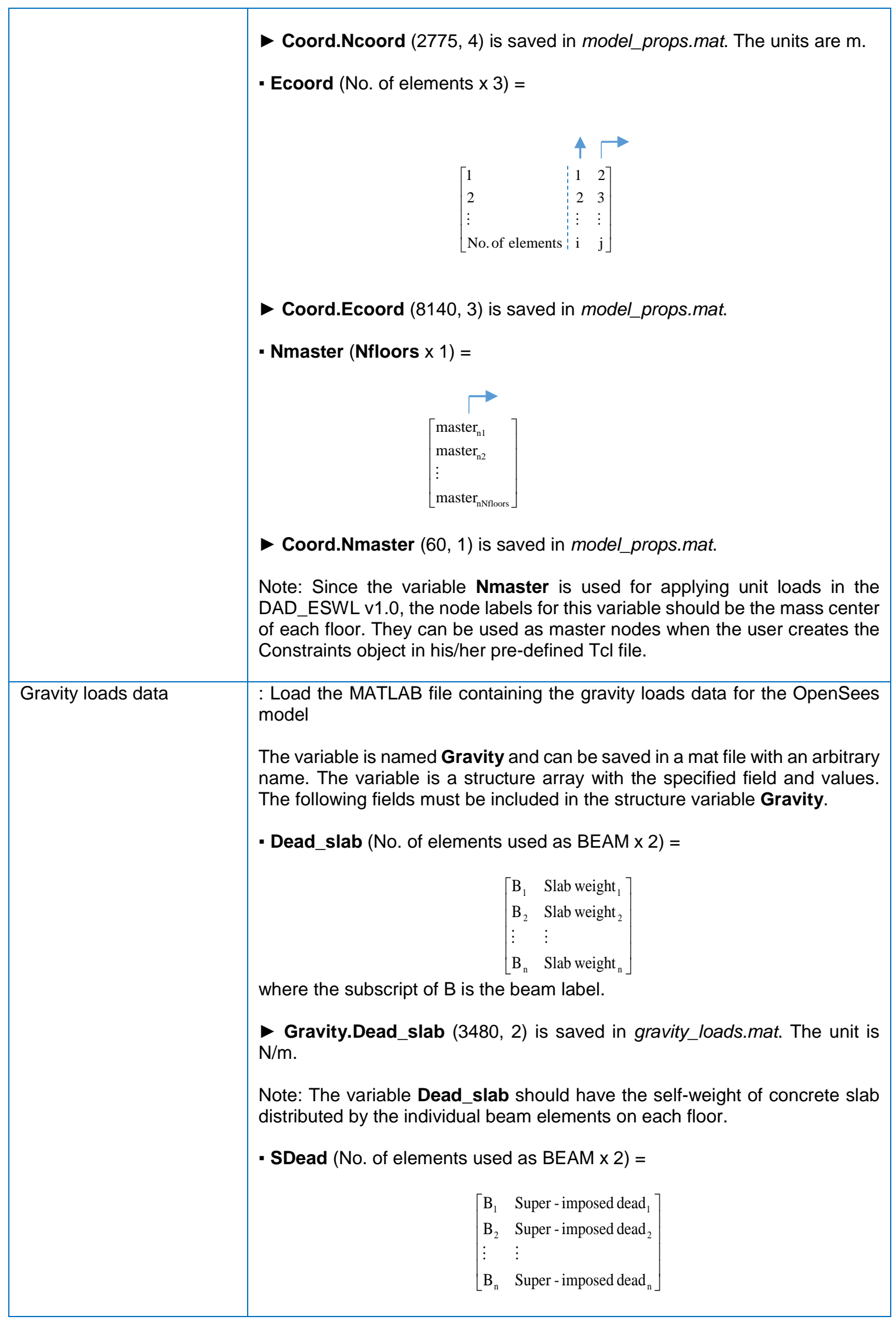




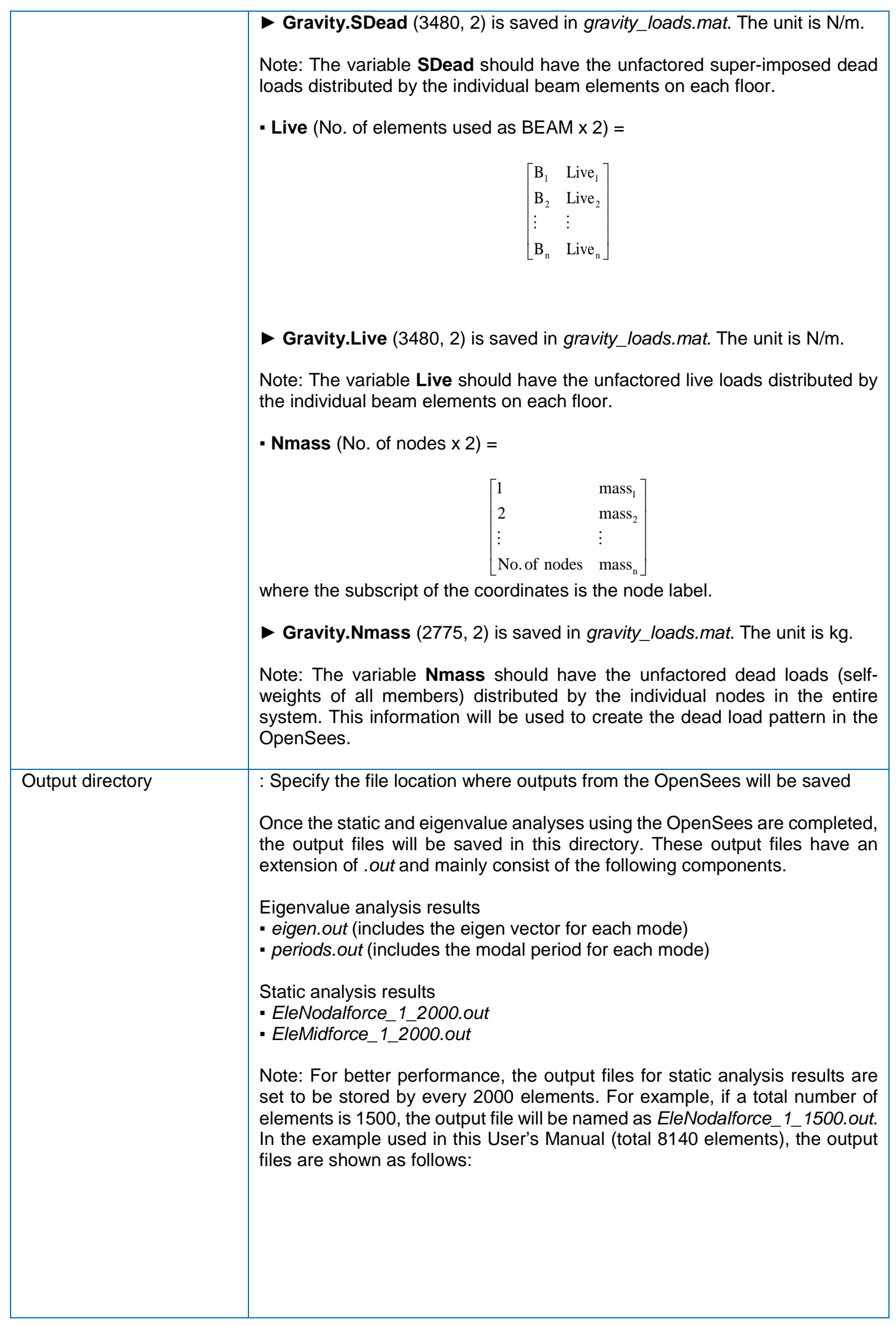




\begin{tabular}{|c|c|c|}
\hline & eigen.out & $13 \mathrm{~KB}$ \\
\hline (3) & EleMidforce_1_2000.out & $8 \mathrm{~KB}$ \\
\hline (2) & EleMidforce_2001_4000.out & $56 \mathrm{KE}$ \\
\hline (1) & EleMidforce_4001_6000.out & \\
\hline (3) & EleMidforce_6001_8000.out & \\
\hline (1) & EleMidforce_8001_8140.out & \\
\hline (3) & EleNodalforce_1_2000.out & \\
\hline (3) & EleNodalforce_2001_4000.out & \\
\hline (1) & EleNodalforce_4001_6000.out & 2 \\
\hline (1) & EleNodalforce_6001_8000.out & 21 \\
\hline (1) & EleNodalforce_8001_8140.out & \\
\hline (1) & periods.out & \\
\hline
\end{tabular}

These output files will be used to make the variables such as dif, frames_DL, frames_SDL, frames_LL, evectors, and T_str. These variables are saved in OpenSees_rslt.mat. 
$\square$ Wind loads

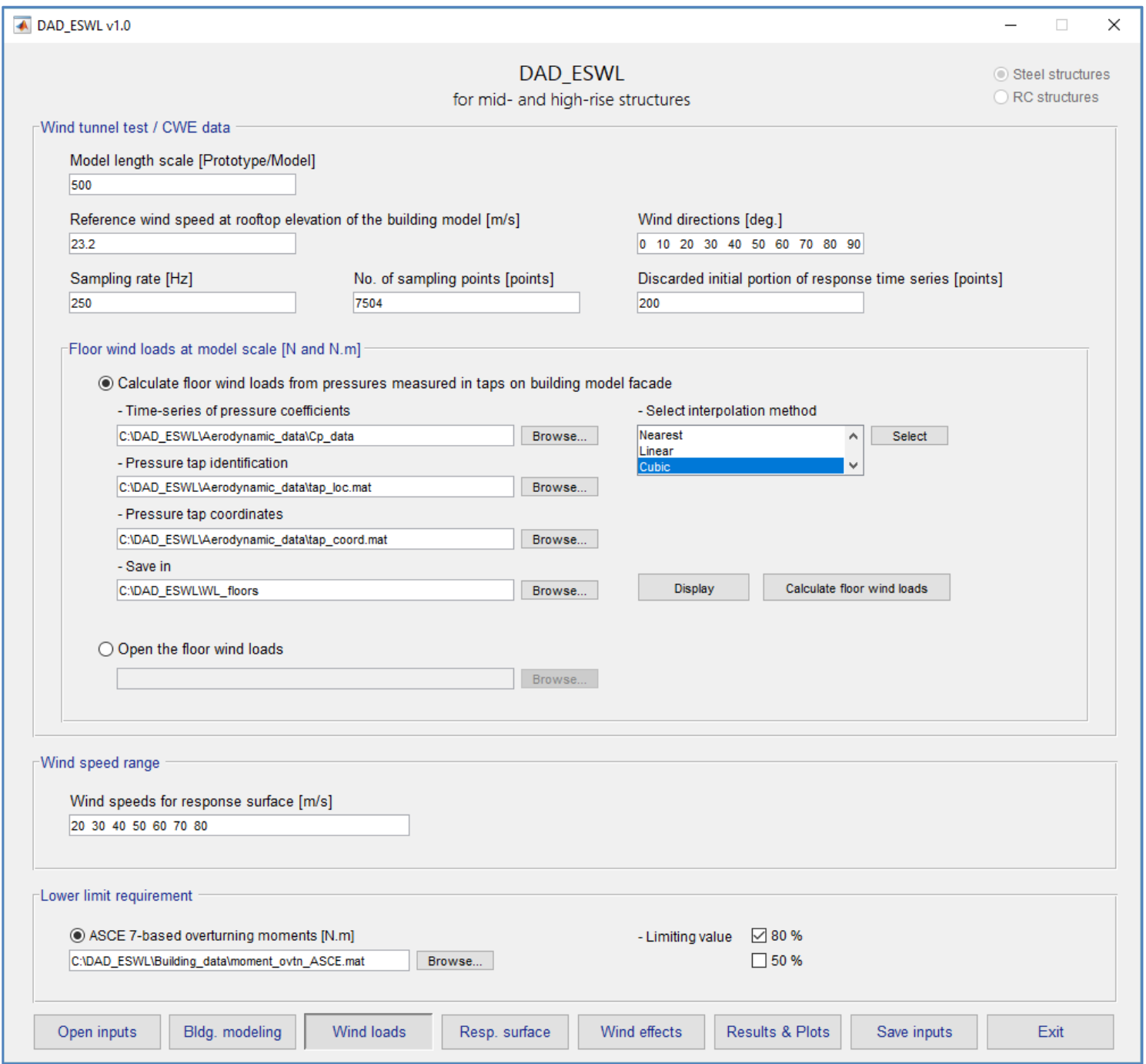

\section{Wind tunnel test I CFD data}

\begin{tabular}{|l|l|}
$\begin{array}{l}\text { Model length } \\
\text { scale } \\
\text { [Prototype/Model] }\end{array}$ & $\begin{array}{l}\text { : Scale of the model used in the wind tunnel tests or CFD simulations; if the scale of } \\
\text { the model to the prototype structure is } 1 / 500 \text {, the value is } 500\end{array}$ \\
\hline $\begin{array}{l}\text { Reference wind } \\
\text { speed at rooftop } \\
\text { elevation of the } \\
\text { building } \\
\text { model[m/s] }\end{array}$ & $\begin{array}{l}: \text { Mean hourly wind speed at roof of the model during wind tunnel tests or CFD } \\
\text { simulations }\end{array}$ \\
\hline $\begin{array}{l}\text { Wind directions } \\
\text { [deg.] }\end{array}$ & $\begin{array}{l}\text { : Wind directions used in the wind tunnel test } \\
\text { The variable WD can be a vector containing clockwise directions of wind as shown in } \\
\text { Fig. } 7 \mathrm{~d} \text {. For example, WD }=0^{\circ} \text { to } 360^{\circ} \text { with } 10^{\circ} \text { clockwise increments from an arbitrary } \\
\text { direction. } \\
\end{array}$ \\
\hline [0:10:360]
\end{tabular}




\begin{tabular}{|c|c|}
\hline & $\begin{array}{l}\text { Note: The wind directions in WD are identical to the wind directions in the wind tunnel } \\
\text { tests or CFD simulations. }\end{array}$ \\
\hline $\begin{array}{l}\text { Sampling rate } \\
{[\mathrm{Hz}]}\end{array}$ & $\begin{array}{l}\text { : Sampling frequency used in the wind tunnel tests or CFD simulations } \\
-250(\mathrm{~Hz})\end{array}$ \\
\hline $\begin{array}{l}\text { No. of sampling } \\
\text { points [points] }\end{array}$ & $\begin{array}{l}\text { : Total number of points that make up the time histories of the floor loads } \\
\text { Npoints }=7504 \text { (from data of } 30 \mathrm{~s} \text { with a sampling frequency of } 250 \mathrm{~Hz} \text { in the wind } \\
\text { tunnel) }\end{array}$ \\
\hline $\begin{array}{l}\text { Discarded initial } \\
\text { portion of } \\
\text { response time } \\
\text { series [points] }\end{array}$ & $\begin{array}{l}\text { : Number of points to be cut from beginning of the time histories during the analysis } \\
200 \\
\text { Note) Numerical integration needs a certain number of points before it stabilizes. } \\
\text { Therefore, a certain number of initial points must be cut from the solution of the } \\
\text { equations of motion before estimating the response parameters. }\end{array}$ \\
\hline \multicolumn{2}{|c|}{ Floor wind loads at model scale [N and N.m] } \\
\hline \multicolumn{2}{|c|}{$\begin{array}{l}\text { Calculate floor wind loads from pressures measured in taps on building model facade } \\
\text { - This option allows the users to load pressure coefficients measured on taps from wind tunnel tests or CFD } \\
\text { simulations. The DAD_ESWL v1.0 will then calculate wind loads applied on mass center of each floor by } \\
\text { using the user's pressure data. }\end{array}$} \\
\hline $\begin{array}{l}\text { Time-series of } \\
\text { pressure } \\
\text { coefficients }\end{array}$ & 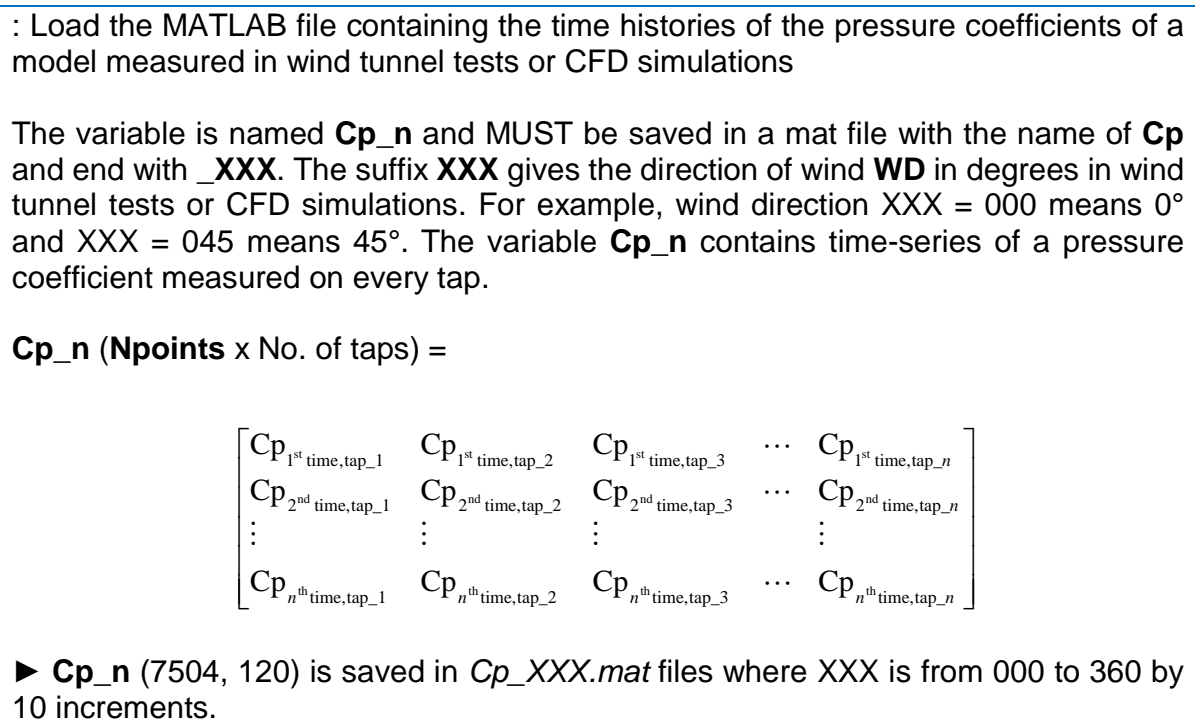 \\
\hline $\begin{array}{l}\text { Pressure tap } \\
\text { identification }\end{array}$ & $\begin{array}{l}\text { : Load the MATLAB file containing the pressure tap identification on building model } \\
\text { facade } \\
\text { The variables are named as WF_1, WF_2, WF_3, and WF_4 and can be saved in a } \\
\text { mat file with an arbitrary name. The suffixes } 1 \text { to } 4 \text { represent the building's face } \\
\text { numbers which are in order of south, east, north, and west of the building face. The } \\
\text { variables are matrices that consist of rows of the number of taps in the building's height } \\
\text { direction, and of columns of the number of taps in the building's width direction. Each } \\
\text { component of this variable should be a tap number. See the example figure below for } \\
\text { better understand. }\end{array}$ \\
\hline
\end{tabular}




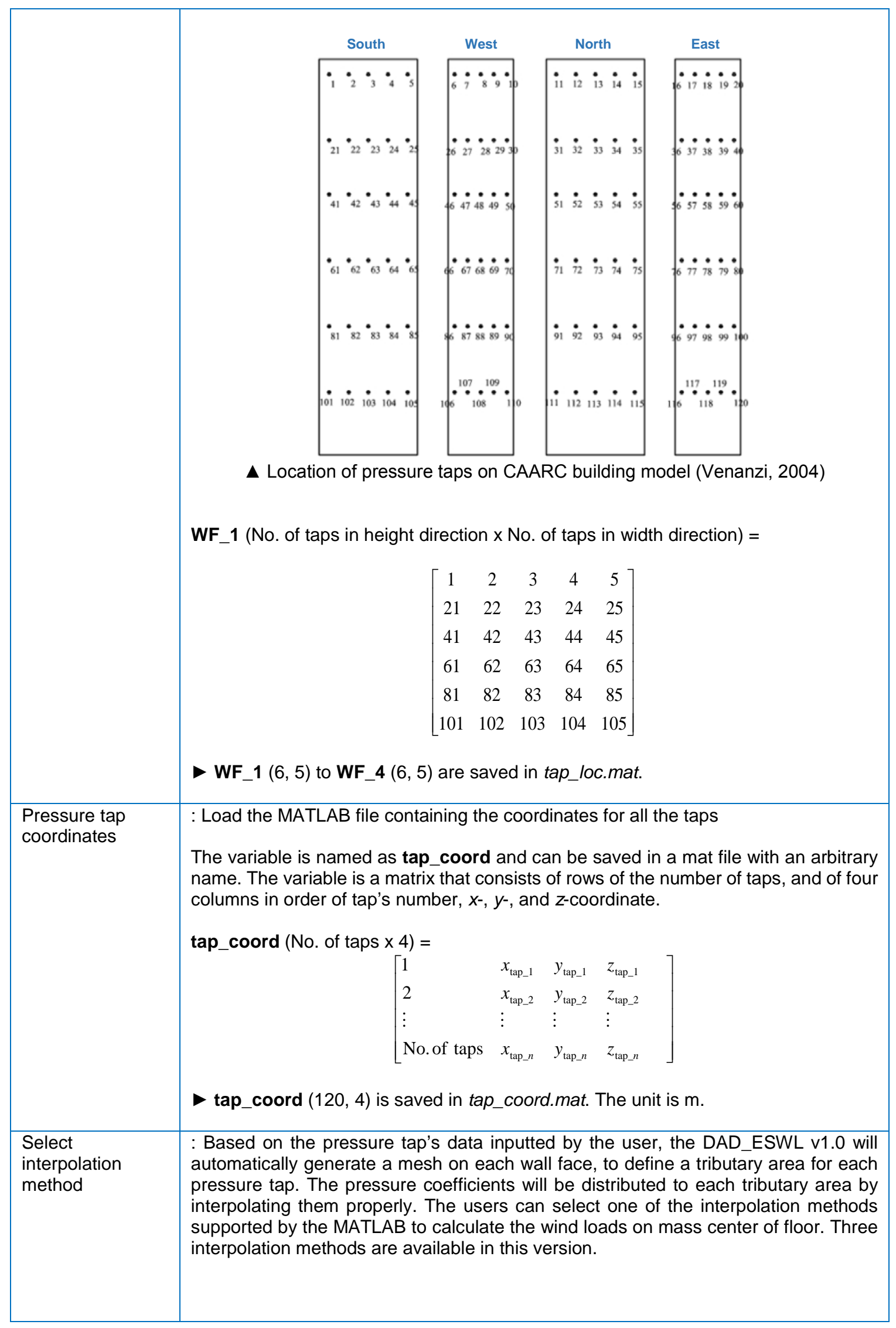




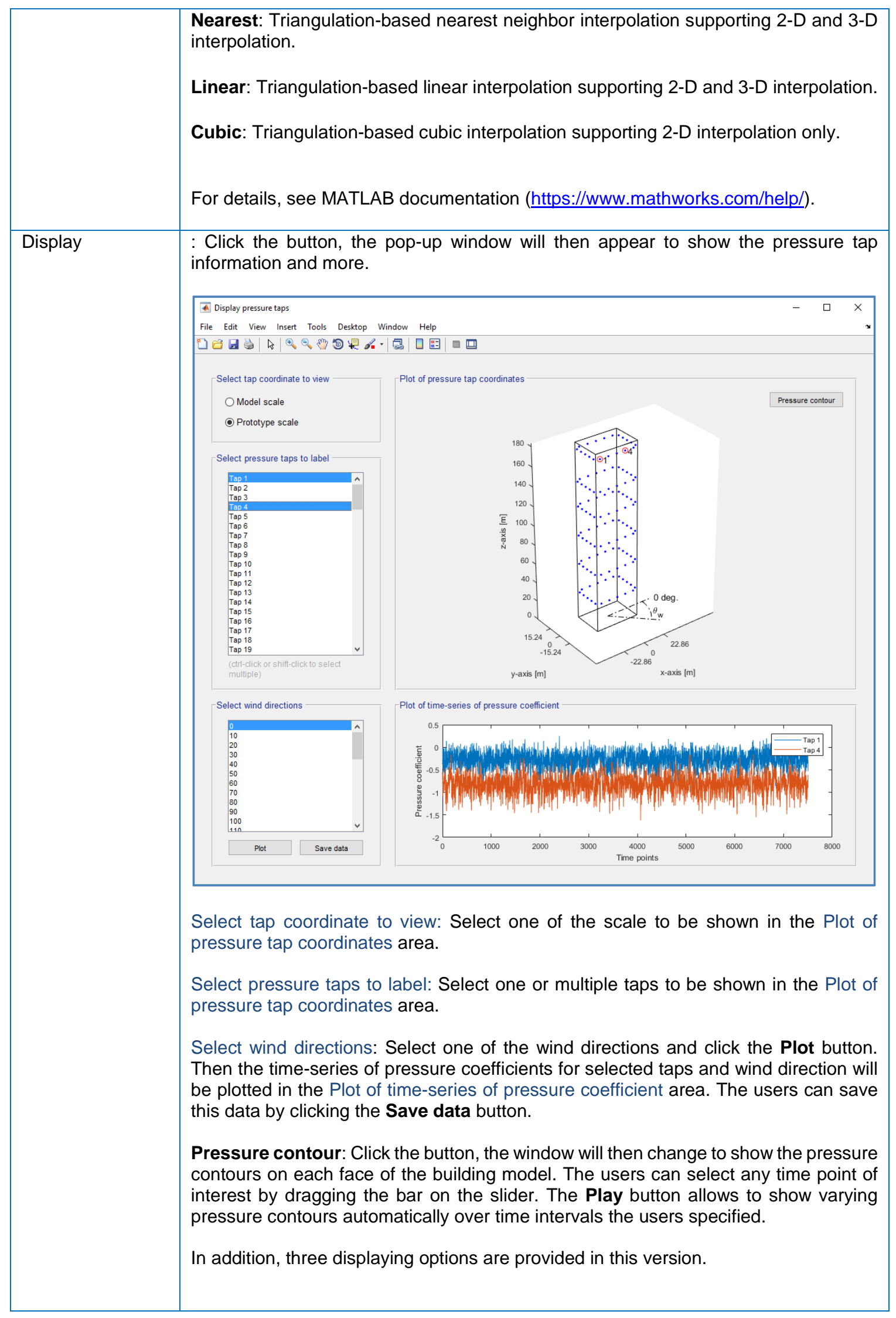




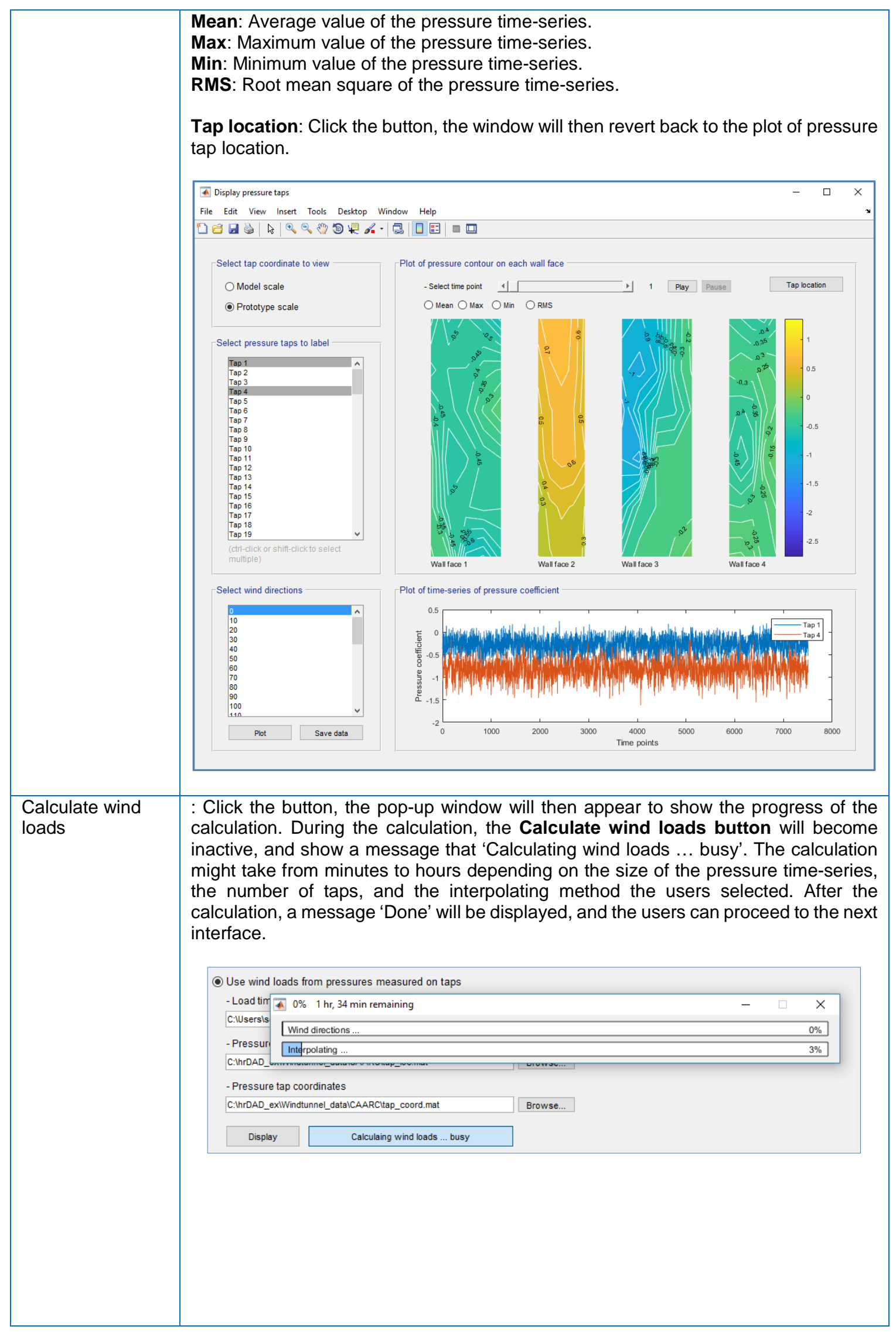




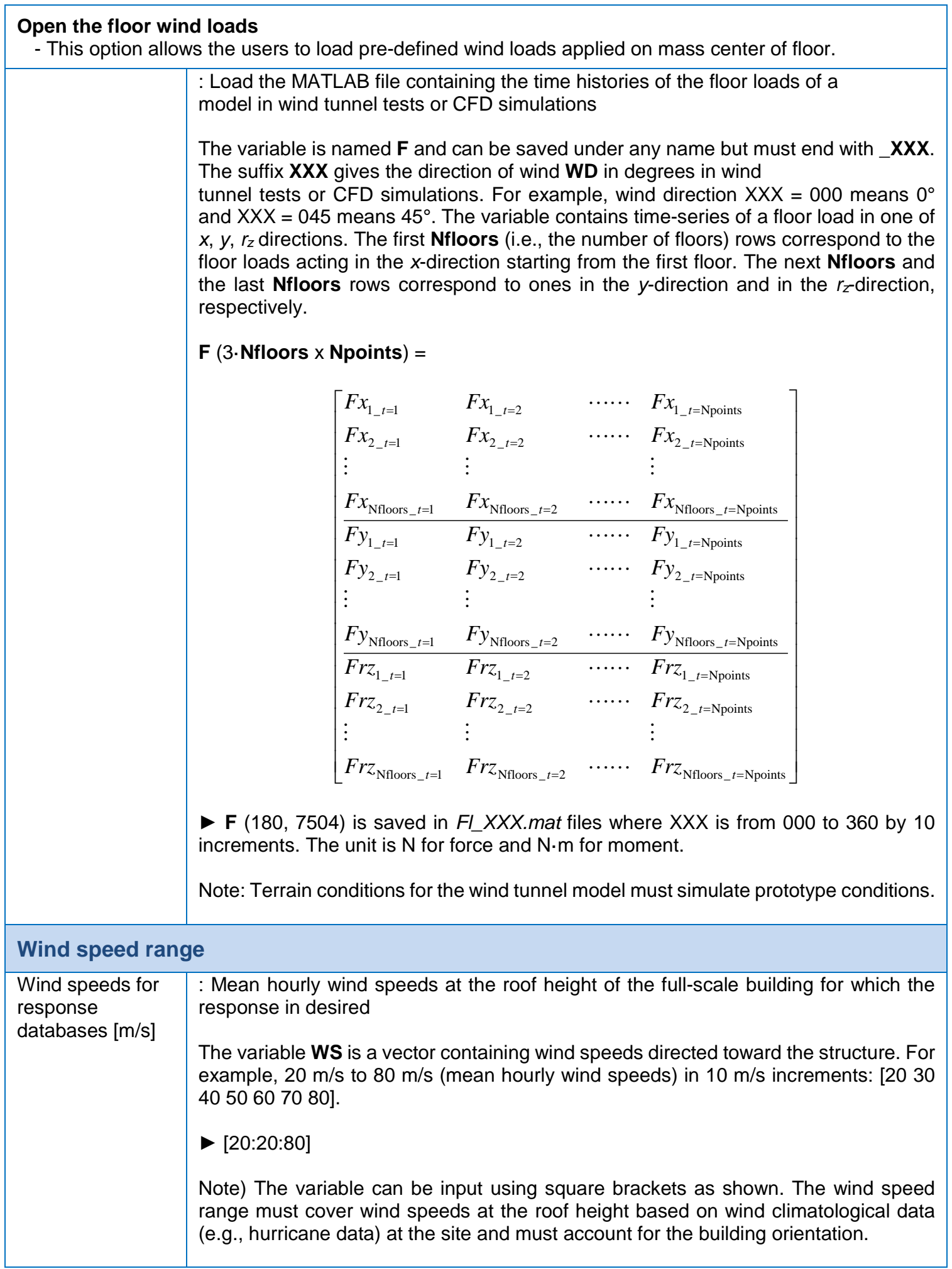




\section{Lower limit requirement}

ASCE 7-based overturning moments $[\mathrm{N} \cdot \mathrm{m}]$
: ASCE 7-based overturning moments in the principal axes corresponding to Risk Category of building

According to ASCE 7-16, Section 31, forces and pressures estimated through wind tunnel testing are to be limited to not less than $80 \%$ or $50 \%$ of their ASCE 7-based analytical method counterpart. DAD_ESWL v1.0 employs overturning moments in the directions of the principal axes as measures of this limitation. Overturning moments obtained from the ASCE 7 analytical method are based on the MRI (Mean Recurrence Interval) corresponding to the Risk Category of the building: 300 years for Risk Category I buildings, 700 years for Risk Category II buildings, and 1700 years for Risk Category III or IV buildings. These overturning moments are compared to their DAD_ESWL v1.0 counterparts.

The variable is named Movtn_asce and can be saved in a mat file with an arbitrary name. The variable Movtn_asce is a matrix in which the first column contains overturning moments along the first principal axis (i.e., $x$-axis) and the second column contains moments along the second principal axis (i.e., $y$-axis). The first row corresponds to ASCE 7-based overturning moments for the first Risk Category being considered for the building. If two or three Risk Categories are considered, they are reflected in the second, or second and third row.

Movtn_asce (No. of MRIs in DCI x No. of principal axes) =

$$
\left[\begin{array}{ll}
M_{x 1} & M_{y 1} \\
M_{x 2} & M_{y 2} \\
\vdots & \vdots \\
M_{x n} & M_{y n}
\end{array}\right]
$$

Movtn_asce $(3,2)$ is saved in moment_ovtn_ASCE.mat where ASCE 7-based overturning moments in the $x$ and $y$ axes based on three Risk Categories of ASCE 7 correspond to the counterpart calculated by DAD_ESWL v1.0. The unit is $\mathrm{N} \cdot \mathrm{m}$.

Limiting value
: Per ASCE 7-16, Section 31, the limiting value of 80 percent may be reduced to 50 percent for the main wind force resisting system. Depending on the conditions of the target model, the user can select one between two limiting values of $80 \%$ and $50 \%$ by clicking the checkbox. 


\section{Resp. surface}

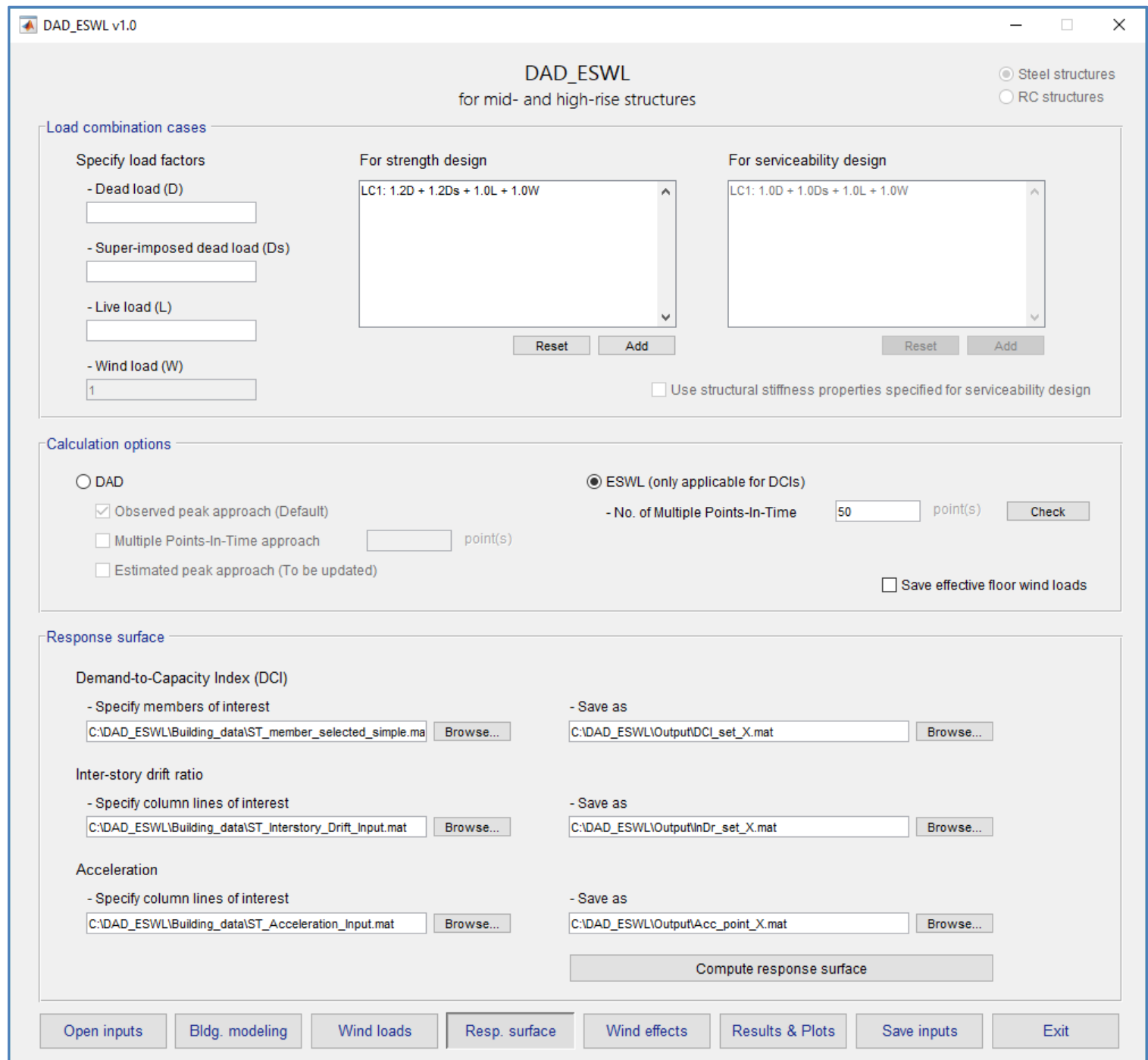

\section{Load combination cases}

\begin{tabular}{|l|l|}
\hline Specify load factors & $\begin{array}{l}\text { : Specify load factors of dead load (D), super-imposed dead load (Ds), live load } \\
\text { (L), and wind load (W) in a load combination of factored loads. } \\
\text { Once the user input the load factors, he/she can make a load combination case } \\
\text { by clicking the Add button. The inputted load combination cases are shown in } \\
\text { Load combination cases list box and are numbered automatically by the order } \\
\text { that the user clicks the Add button. }\end{array}$ \\
$\begin{array}{l}\text { The Reset button allows the users to reset all the load combination cases. } \\
\text { Note 1) Wind load factor is fixed to 1. } \\
\text { Note 2) Load combination cases for serviceability design (inter-story drift ratio } \\
\text { and acceleration) cannot be modified. By default, all the load factors are fixed to } \\
1 .\end{array}$ \\
\hline
\end{tabular}




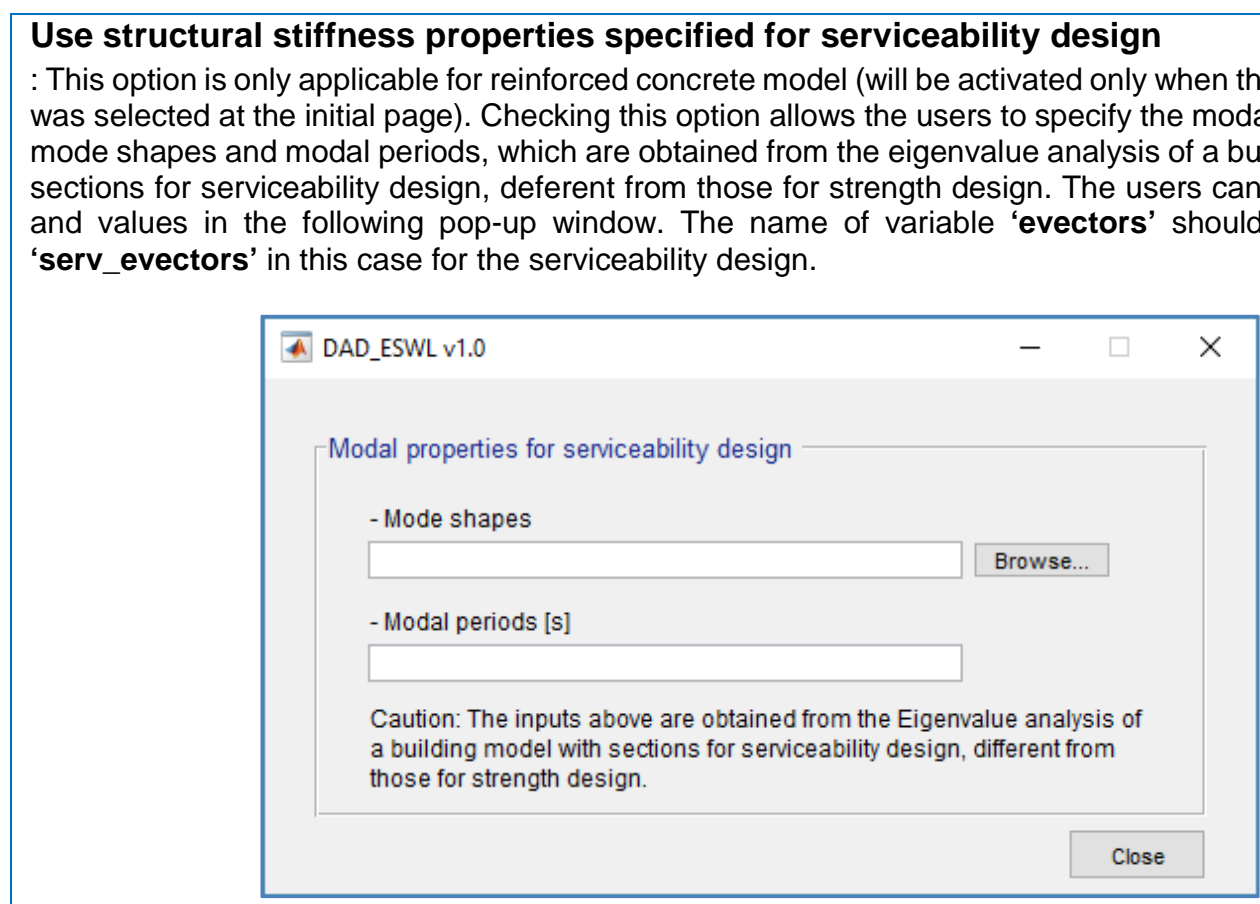

\section{Use structural stiffness properties specified for serviceability design}

the RC structures can input those files and values in the following pop-up window. The name of variable 'evectors' should be changed to 'serv_evectors' in this case for the serviceability design. 


\section{Calculation options}

\section{DAD (responses determined by fluctuating loads)}

: Enables to use the DAD procedure for producing DCl response surface.

\begin{tabular}{|l|l|}
\hline $\begin{array}{l}\text { Observed peak } \\
\text { approach }\end{array}$ & $\begin{array}{l}\text { : By default, an observed peak approach (a full-time approach) is set for } \\
\text { calculating the DCI response surface. }\end{array}$ \\
\hline $\begin{array}{l}\text { Multiple Points-In-Time } \\
\text { approach }\end{array}$ & $\begin{array}{l}\text { : Check this option to use the multiple points-in-time approach (MPIT) for the } \\
\text { calculation of the DCI response surface. Once this is activated, specify the } \\
\text { number of points used in the MPIT approach. } \\
\text { Note) The point-in-time approach considerably reduces calculation time while } \\
\text { maintaining the accuracy. For details, see Yeo (2013). }\end{array}$ \\
\hline $\begin{array}{l}\text { Estimated peak } \\
\text { approach }\end{array}$ & $\begin{array}{l}\text { : Estimated peak approach will be updated in later version of DAD_ESWL. } \\
\text { Equivalent Static Wind Load (ESWL) } \\
\text { : Enables to use the ESWL procedure for producing DCI response surface. }\end{array}$ \\
\hline $\begin{array}{l}\text { No. of Multiple Points- } \\
\text { In-Time }\end{array}$ & $\begin{array}{l}: \text { The number of MPIT should be inputted by the users. This should be a positive } \\
\text { integer which is much less than the total sampling points. }\end{array}$ \\
\hline $\begin{array}{l}\text { Check } \\
\text { : Check button allows the users to check how many wind loading cases will be } \\
\text { generated for ESWL procedure. For example, if the number of MPIT is set as 50, } \\
\text { total 300 equivalent wind loading cases (WLCs) will be generated. For details, } \\
\text { see Park et al. (2018). Clicking this button after specifying the number of MPIT } \\
\text { activates a small pop-up window as shown below. }\end{array}$
\end{tabular}

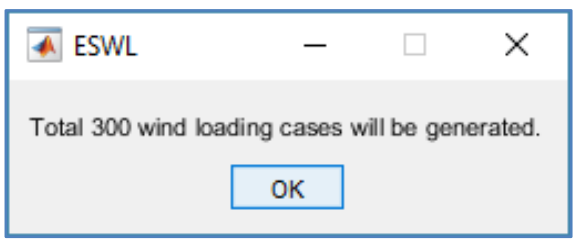

\section{Save effective floor wind loads}

: Checking this option allows the users to save the effective floor wind loads generated during the analyses. If the user selects the DAD option above, the time series of effective floor wind loads will be saved at the user-specified directory. If the ESWL option is selected, the effective floor wind loads with respect to the wind loading cases will be saved. For details, see the next section for computing response surfaces.

\section{Response surface}

\section{Demand-to-Capacity Index (DCI)}

Specify members of interest
: Load the MATLAB file containing list of members to calculate their demand-tocapacity indexes.

The variable is named member_selected and can be saved in a mat file with an arbitrary name. The variable member_selected is a one-row vector that contains a list of the members of interest for which response database for the demand-to-capacity index are calculated.

member_selected $(1 \times$ No. of members of interest $)=$

$$
\left[\begin{array}{llllll}
1 & 301 & \cdots & 901 & \cdots & 2000
\end{array}\right]
$$

member_selected $(1,20)$ is saved in ST_member_selected_simple.mat. The 3 members consisting of the columns, beams, and bracings of interest were 


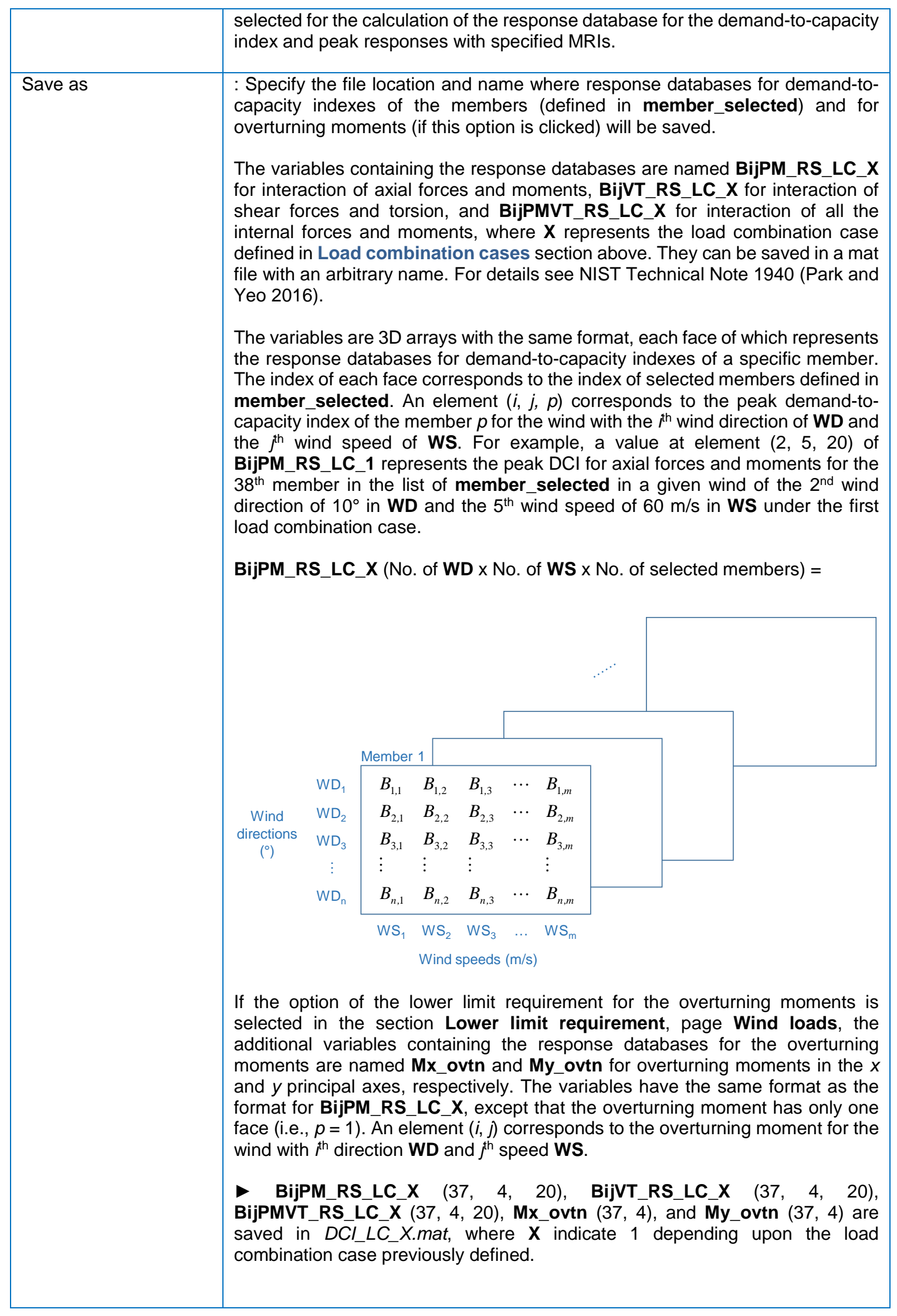




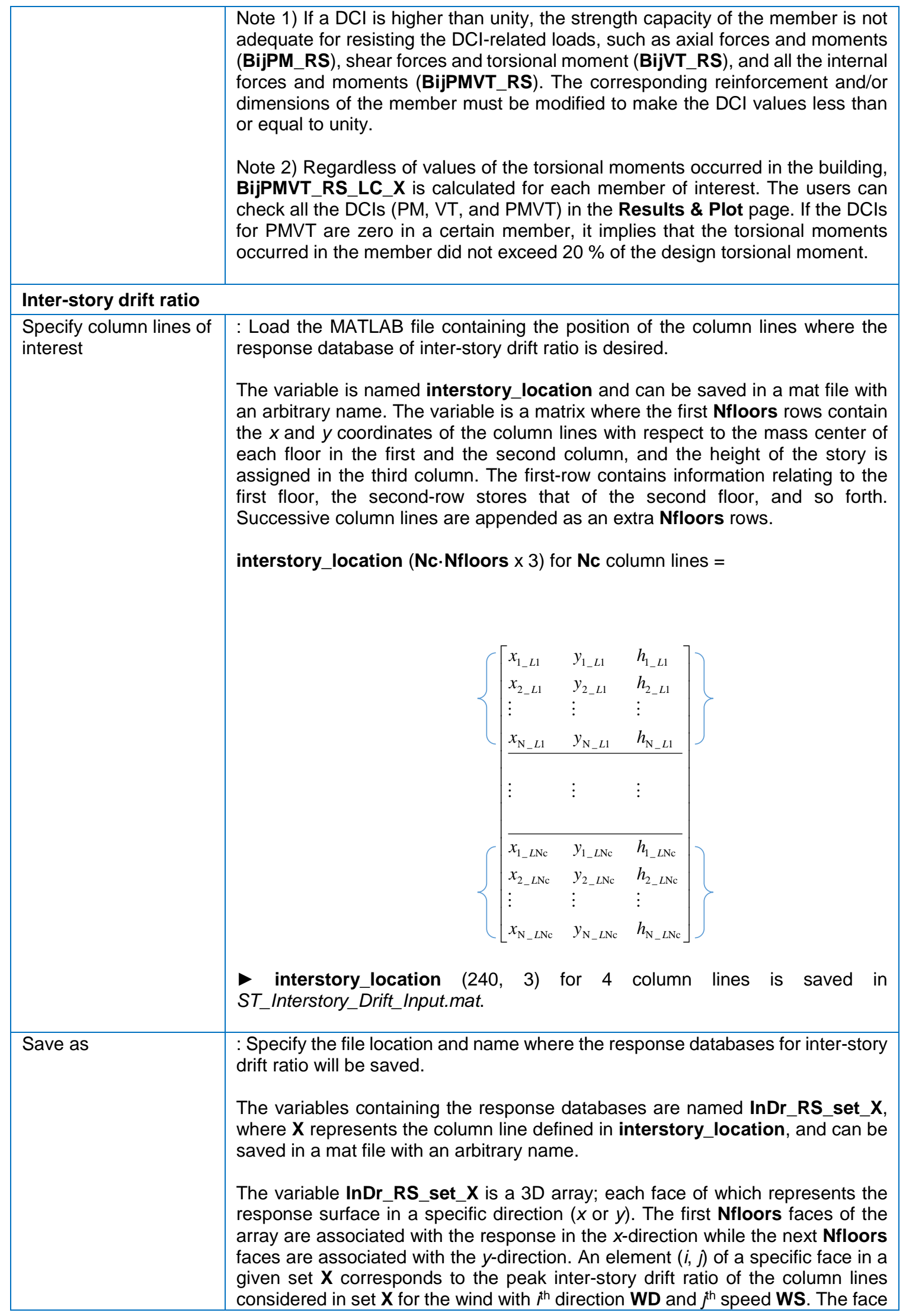




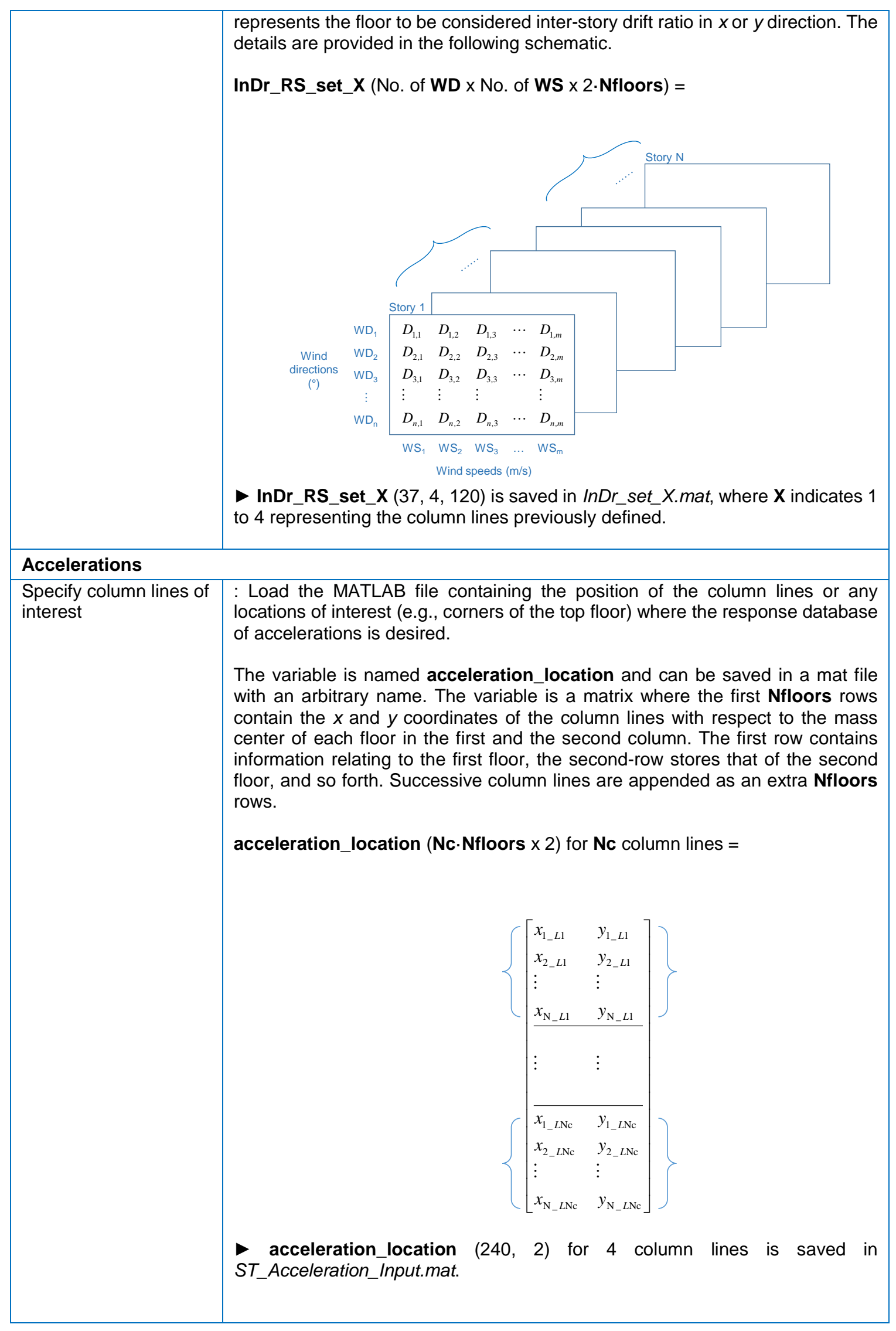




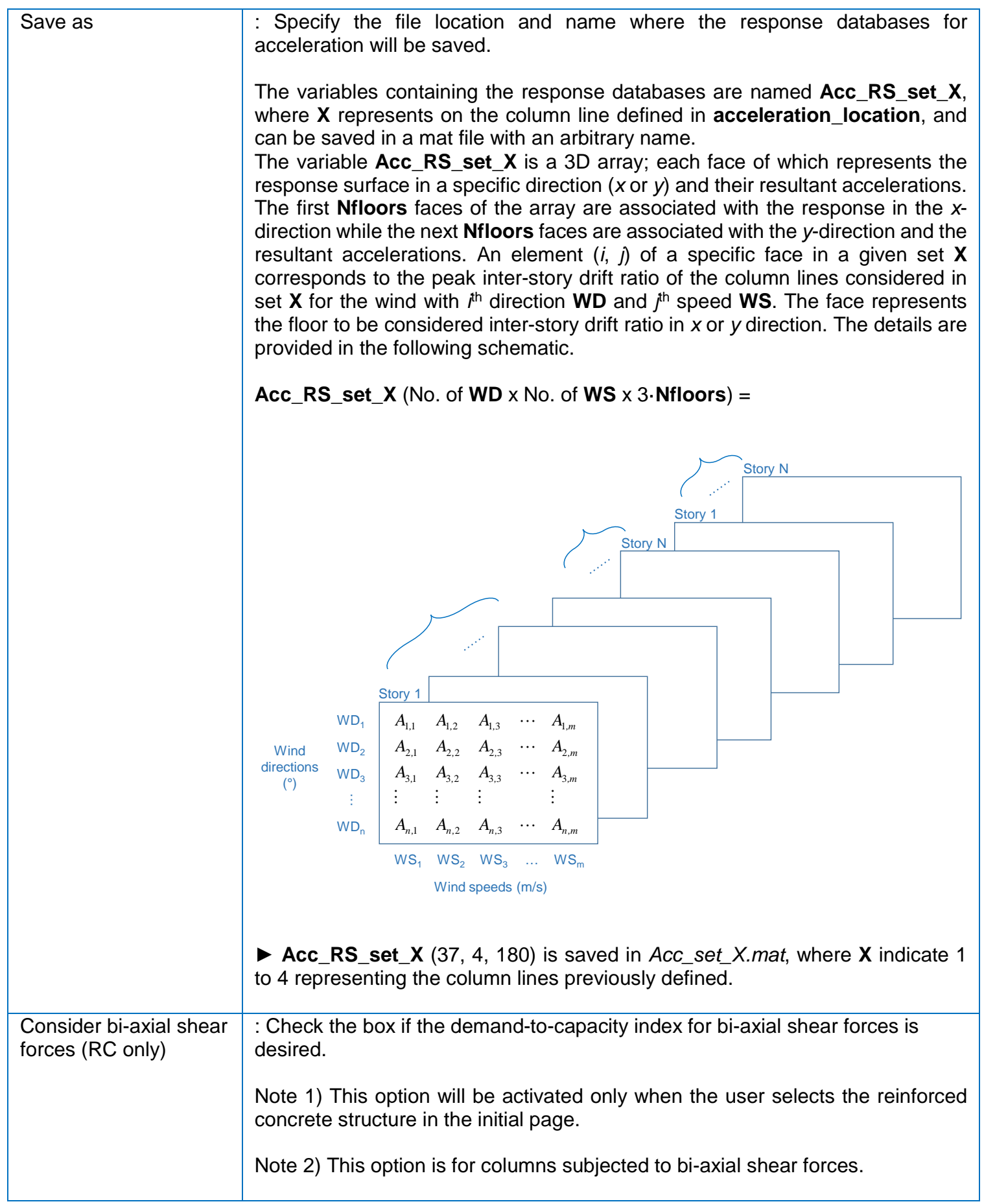




\section{Compute response surface}

: Click the button, and the response surfaces defined above are calculated. The pop-up window will then appear to show the progress of the calculation.

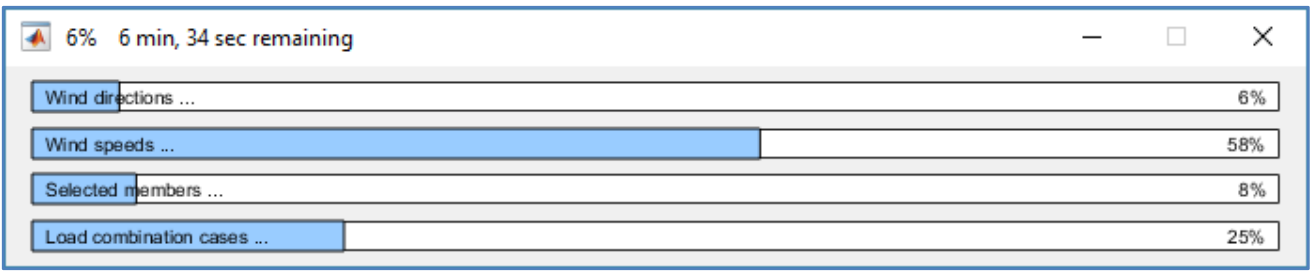

Note 1) The calculations can be performed when all variables are provided correctly in all the previous pages and sections.

Note 2) If the user checked 'Save effective floor wind loads' option in the Calculation options panel, a pop-up window will then appear to choose wind speeds, directions, and path to save the file. After selecting and specifying them, click the OK button to proceed. This operation will consume additional time and system resources depending on the wind speeds and directions selected. For reference, it took few more minutes to save all cases, i.e., 7 wind speeds and 36 directions, with output file taking up 9 gigabytes by using a personal computer.

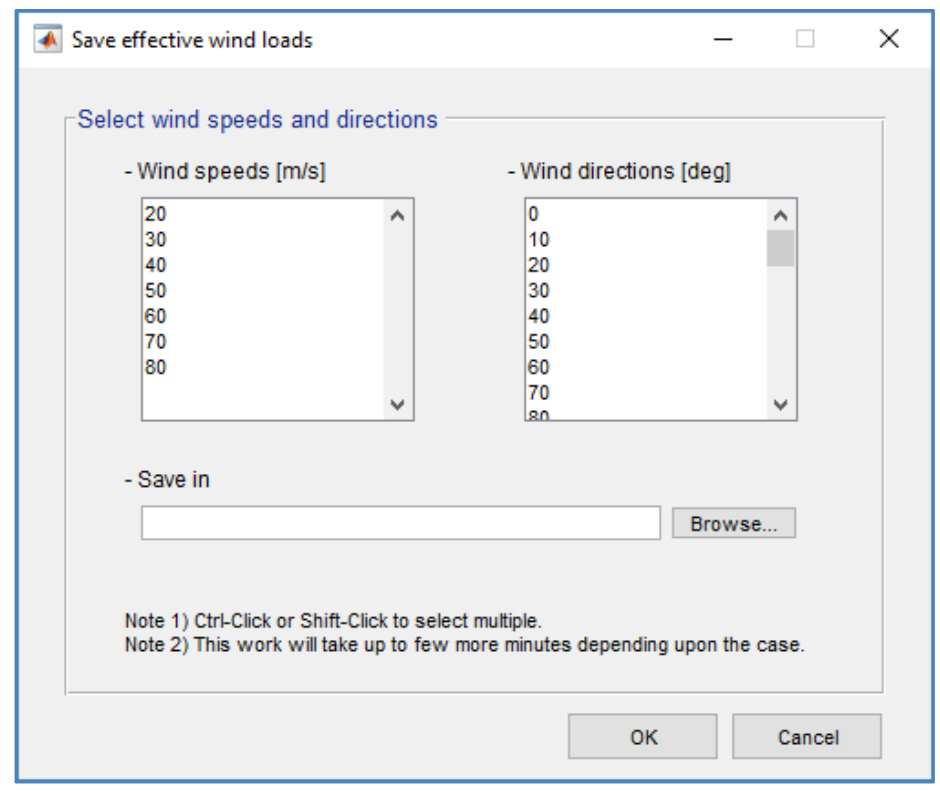


$\square$ Wind effects

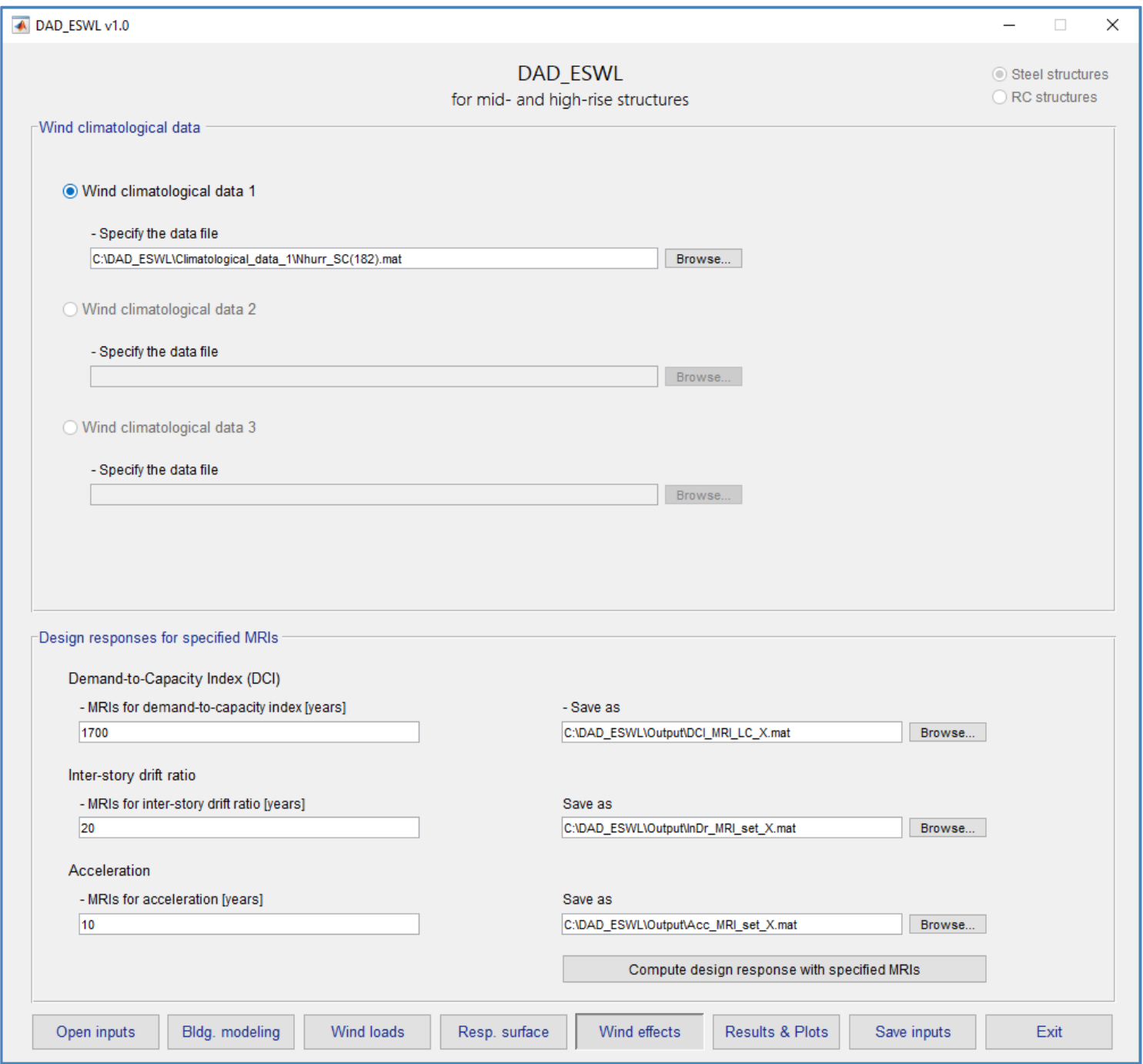

\section{Wind climatological data}

Note 1) The wind engineering laboratory provides extreme directional wind speeds for the following default conditions: mean wind speed averaged over approximately $30 \mathrm{~min}$ to one hour at the elevation above ground of the top of the building at the empty (pre-construction) building site. If wind speeds corresponding to other conditions are available, they must be converted to mean speeds for the default conditions.

Note 2) Any different types of the extreme directional wind speed data, i.e., hurricanes, thunderstorms, and synoptic winds, can be used via 'Wind climatological data 1', 'Wind climatological data 2', and 'Wind climatological data 3' options below.

Note 3) The users can select up to THREE of these options at the same time for investigating a mixed climate effect.

\section{Wind climatological data 1, 2, and 3}

\begin{tabular}{l|l} 
Specify the data file & : Load the MATLAB file containing the database of the extreme directional
\end{tabular} wind speeds 


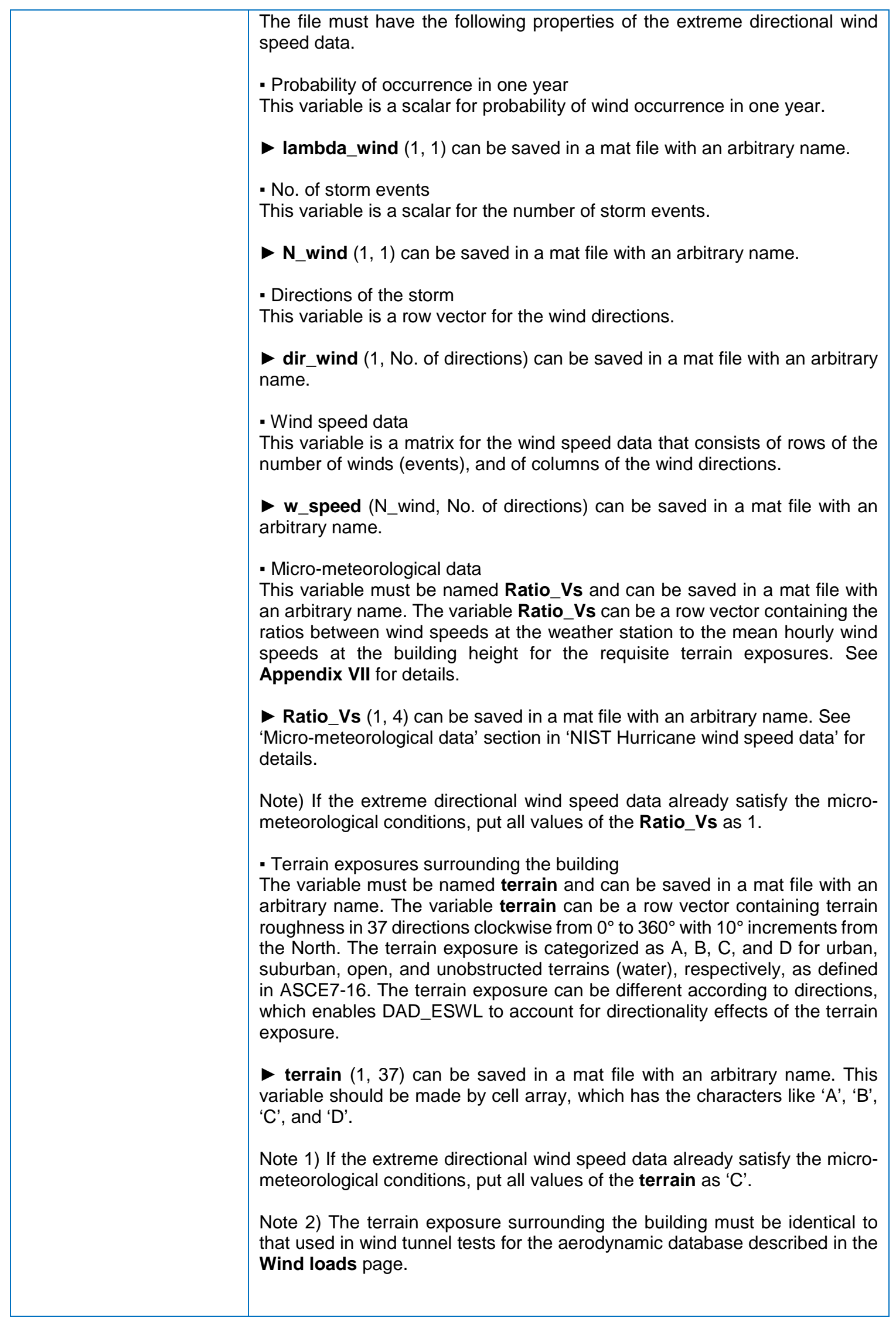




\begin{tabular}{|c|c|}
\hline $\begin{array}{l}\text { MRIs for demand-to- } \\
\text { capacity index [years] }\end{array}$ & $\begin{array}{l}\text { : Mean Recurrence Intervals (MRIs) for demand-to-capacity index (DCl) of } \\
\text { selected members } \\
\text { [1700] } \\
\text { Note) The unit is year. }\end{array}$ \\
\hline Save as & $\begin{array}{l}\text { Specify the file location and name where the peak response of DCls for } \\
\text { selected members with specified MRIs will be saved } \\
\text { The variables containing the selected members' DCIs for axial force and } \\
\text { bending moments and for shear forces and torsional moment with specified } \\
\text { MRIs are named Bij_PM_MRIs_LC_X and Bij_VT_MRIs_LC_X, } \\
\text { respectively, where X represents the load combination case defined in Load } \\
\text { combination cases section and can be saved in a mat file with an arbitrary } \\
\text { name. } \\
\text { These variables are matrices the rows of which contain the selected } \\
\text { members' peak DCls for the MRI specified above. The members of interest } \\
\text { are defined in member_selected in the Response DB page. The first row } \\
\text { will contain the indexes corresponding to the first MRIs for DCI defined above, } \\
\text { the second row will have those corresponding to the second MRI, and so } \\
\text { forth. The index of each column is the index of the members defined in } \\
\text { member_selected. For example, an element (3, 300) of Bij_PM_MRIs_LC_X } \\
\text { represents the peak DCI for axial force and bending moments of the } 300^{\text {th }} \\
\text { member in the list member_selected with the } 3^{\text {rd }} \text { of the MRIs specified for } \\
\text { the DCl. }\end{array}$ \\
\hline
\end{tabular}

Bij_PM_MRIs_LC_X (No. of MRIs, No. of selected members $(=\mathrm{Nm})$ ) for a given load combination case $\mathbf{X}=$

$$
\left[\begin{array}{lllll}
\mathrm{B}_{\mathrm{MRI}=1,1} & \mathrm{~B}_{\mathrm{MRI}=1,2} & \mathrm{~B}_{\mathrm{MRI}=1,3} & \cdots & \mathrm{B}_{\mathrm{MRI}=1, \mathrm{Nm}} \\
\mathrm{B}_{\mathrm{MRI}=2,1} & \mathrm{~B}_{\mathrm{MRI}=2,2} & \mathrm{~B}_{\mathrm{MRI}=2,3} & \cdots & \mathrm{B}_{\mathrm{MRI}=2, \mathrm{Nm}} \\
\vdots & \vdots & \vdots & & \vdots \\
\mathrm{B}_{\mathrm{MRI}=\mathrm{P}, 1} & \mathrm{~B}_{\mathrm{MRI}=\mathrm{P}, 2} & \mathrm{~B}_{\mathrm{MRI}=\mathrm{P}, 3} & \cdots & \mathrm{B}_{\mathrm{MRI}=\mathrm{P}, \mathrm{Nm}}
\end{array}\right]
$$

Bij_PM_MRIs_LC_X $(1,20)$ and Bij_VT_MRIs_LC_X $(1,20)$ of 20 selected members for one MRI are saved in DCI_mri_LC_X.mat, where $\mathbf{X}$ indicates 1 and 2 depending upon the load combination cases previously defined.

Note 1) If the option of the lower limit requirement for the overturning moment is selected in the section Lower limit requirement, page Wind loads, the variables Scaled_Bij_PM_MRIs_LC_X and Scaled_Bij_VT_MRIs_LC_X containing the adjusted peak DCls for the selected members are also saved.

Note 2) Additional variables saved in the file can be used for the post-process analysis:

Mx_ovtn_MRIs (No. of MRIs, 1):

Overturning moments along the $\mathrm{x}$ axis corresponding to the specified MRIs.

My_ovtn_MRIs (No. of MRIs, 1):

Overturning moments along the $y$ axis corresponding to the specified MRIs.

Rt_Mx_ovtn (No. of MRIS, 1): 


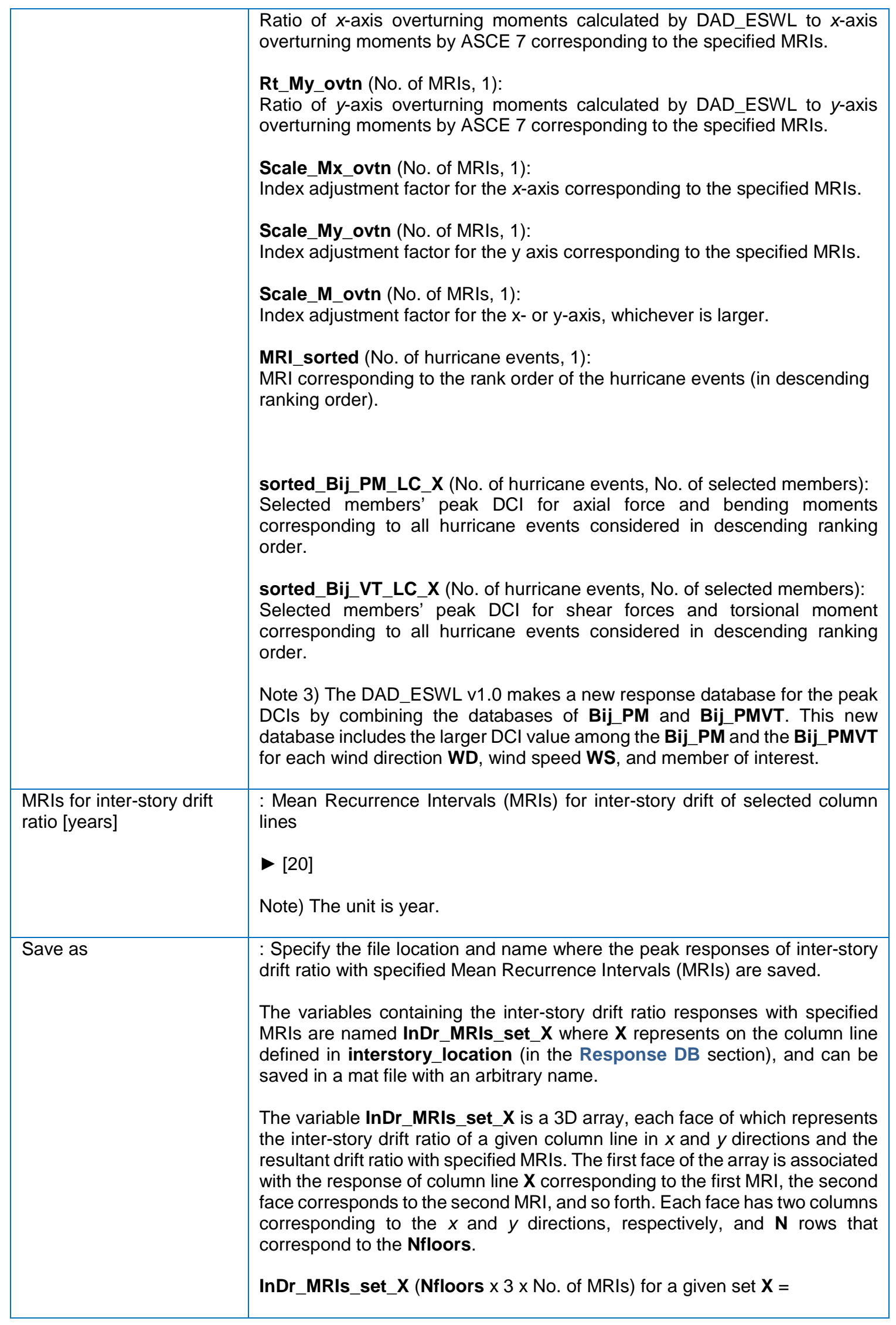




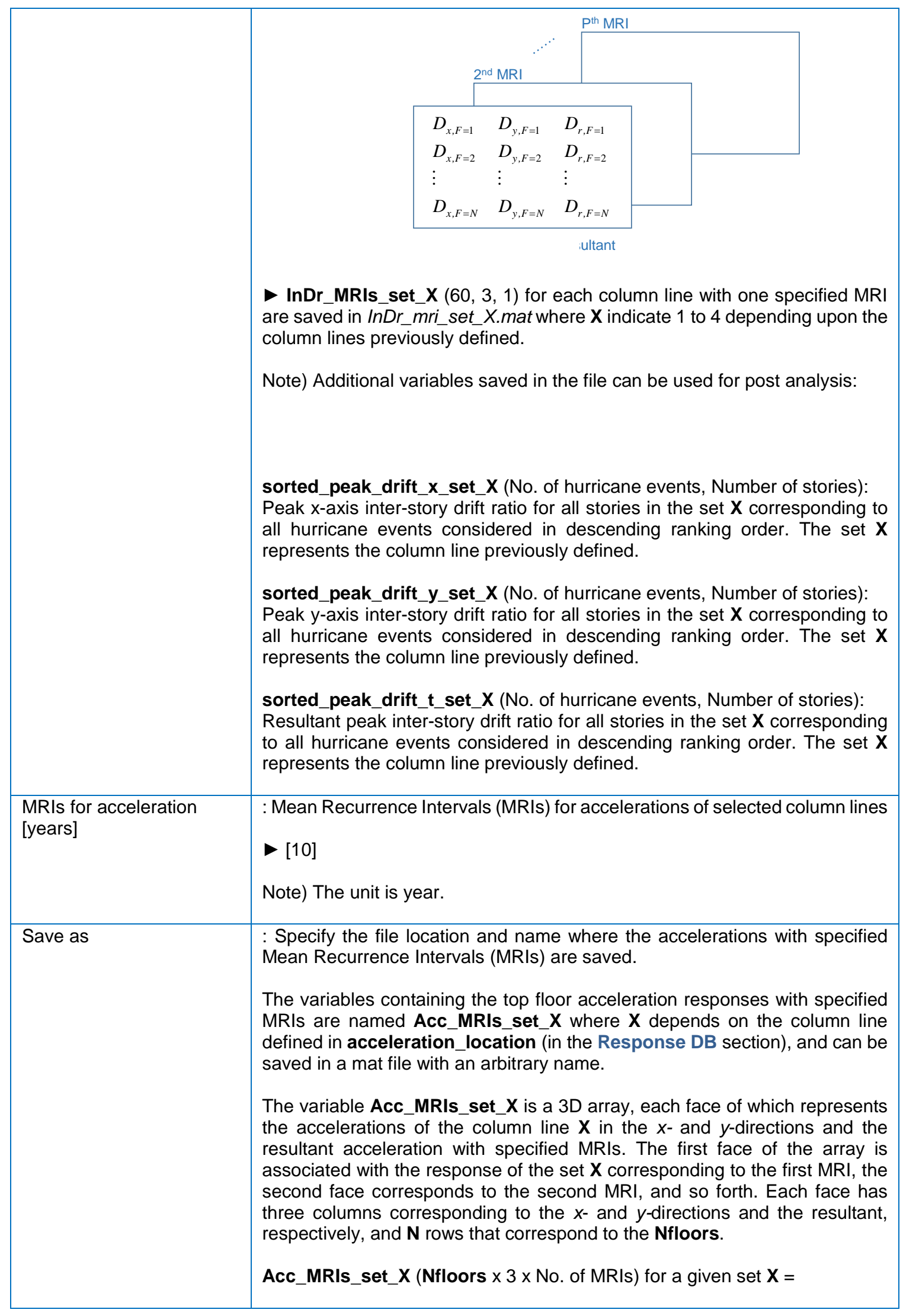




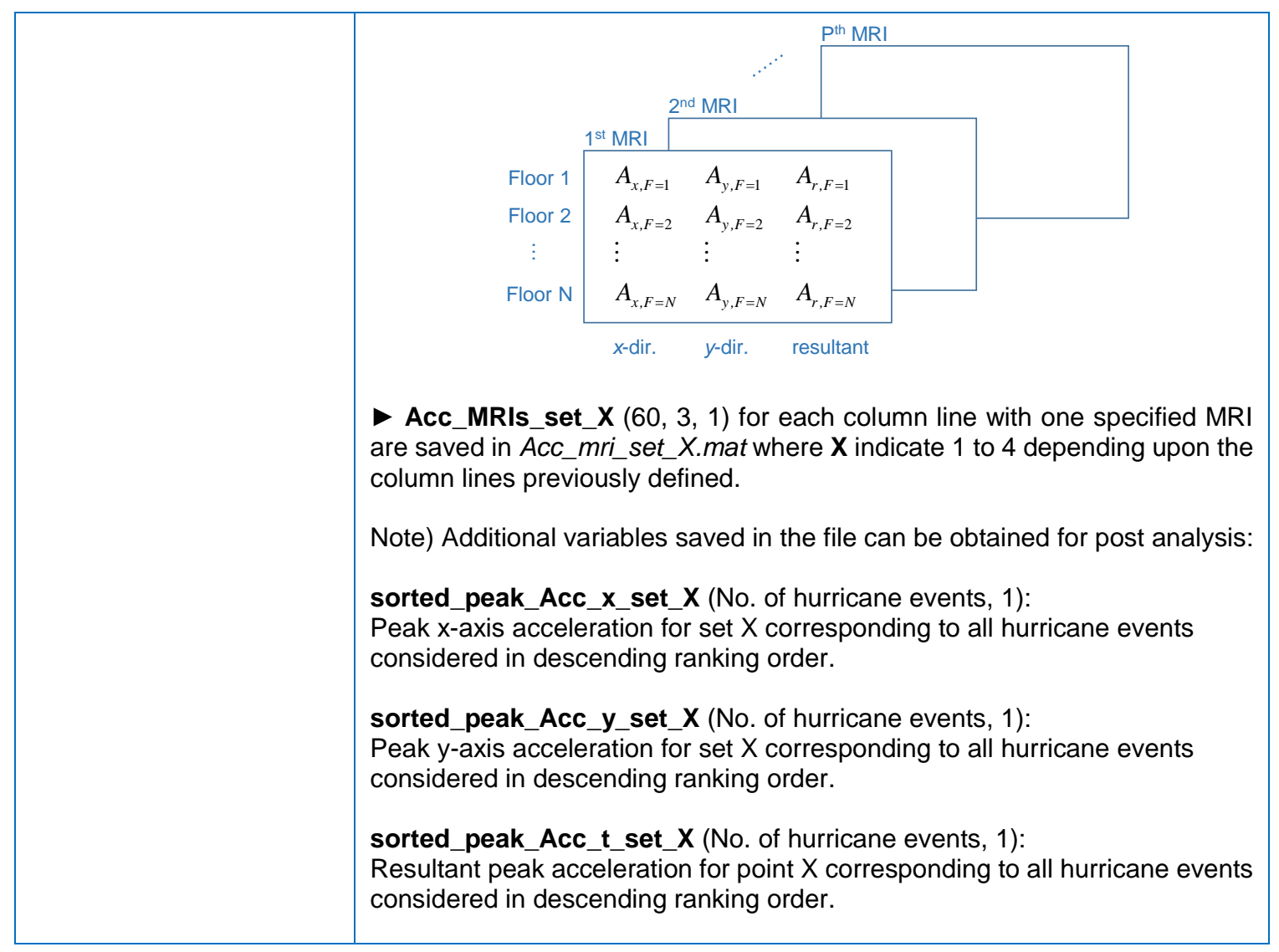




\section{Compute design responses with specified MRIs}

Click the button, and the pop-up window for setting the number of workers for parallel computing will appear as below. The DAD_ESWL will automatically detect and show how many physical cores are in the user's local system, for example, '...2 physical core(s) detected'. By default, this number will be set automatically as the number of workers. If the users want to use more workers for a specific reason, i.e., if their system have more logical cores than physical cores, the users can select the 'Set number of workers' check box and set the number of workers they want to use. After then, click the 'Start computation' button to proceed to compute the design responses with specified MRIs.

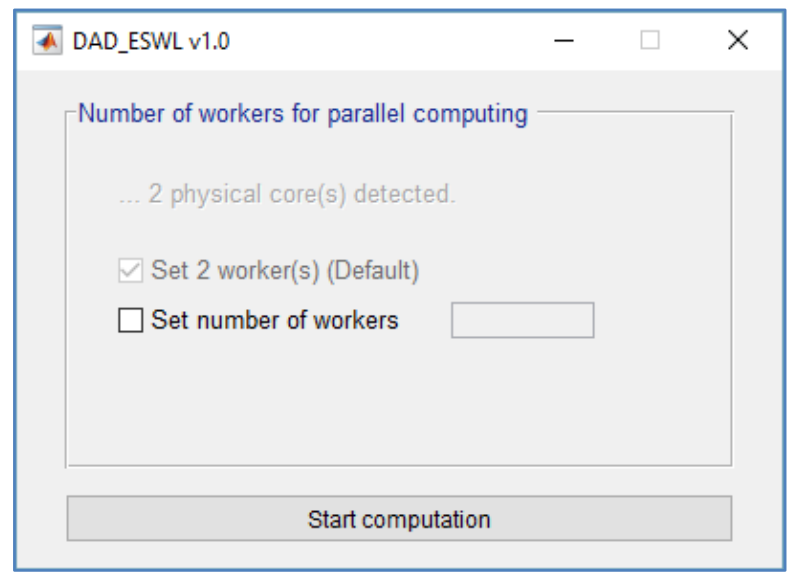

The pop-up window will then appear to show the progress of the computation.

\begin{tabular}{|l|l|}
\hline A $5 \%$ 31 sec remaining & - \\
\hline Load fases ... & \\
\hline Calculating peak DCls of each selected member ... & $5 \%$ \\
\hline Analyzing directional responses ... & $5 \%$ \\
\hline
\end{tabular}

Note) If the response surfaces were not constructed for the wind directions from $0^{\circ}$ to $360^{\circ}$ in increments of $10^{\circ}$, the pop-up window will appear to show warning message as below. In this case, the response surface should be reconstructed to satisfy the wind direction limitation.

\begin{tabular}{|l|}
\hline A DAD_ESWL v1.0 \\
- For the analysis on extreme directional wind speeds, the wind \\
directions from $0^{\circ}$ to $360^{\circ}$, in increments of $10^{\circ}$, should be used \\
for contruction of the response surfaces. \\
- Please go back to [Resp. surface] page, and reconstruct the \\
response surfaces for the wind directions of 0:10:360. \\
\hline Dismiss \\
\hline
\end{tabular}




\section{Results \& Plots}

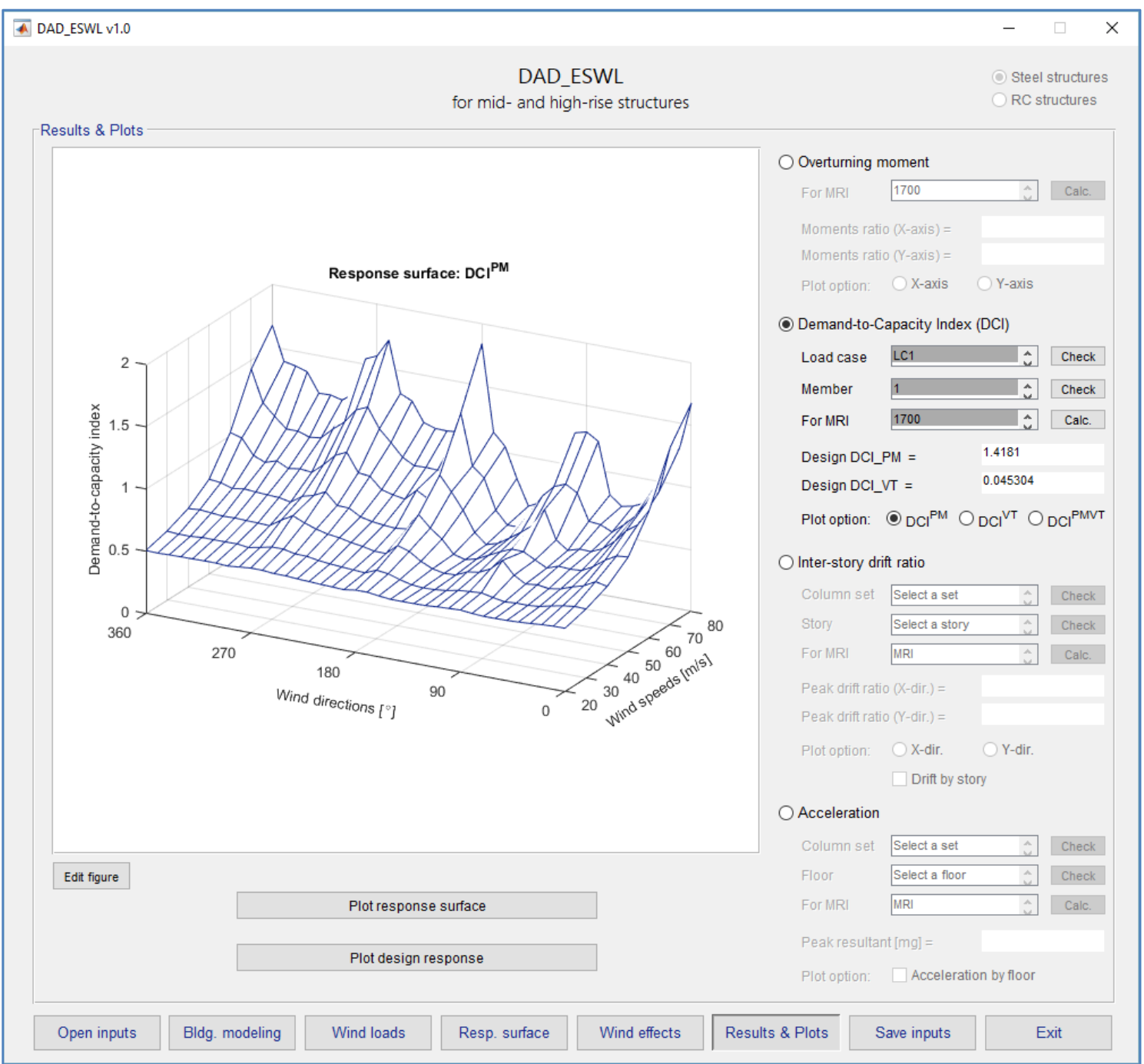

\section{Results \& Plots}

\section{Overturning moment}

Once the button is clicked, the overturning moment module is activated.

Note: If the option of lower limit requirement for overturning moments is not clicked in the section Lower limit requirement of the page Wind load, the overturning moments module is not activated.

\begin{tabular}{|l|l|}
\hline For MRI & $\begin{array}{l}\text { : Select a MRI of interest from the list of MRIs, and click the button of Calc. } \\
\text { Overturning moments in the } X \text {-and } Y \text {-axes for the selected MRIs will be } \\
\text { shown in the blanks. } \\
\text { Note) The list of MRIs for overturning moments is obtained from MRIs for } \\
\text { demand-to-capacity index in the page Wind Effects. }\end{array}$ \\
\hline Plot response database & $\begin{array}{l}\text { : Place the button of Plot response database in the bottom left corner of the } \\
\text { page after a Plot option (X-axis or Y-axis) is chosen }\end{array}$ \\
\hline
\end{tabular}




\begin{tabular}{|c|c|}
\hline & $\begin{array}{l}\text { The response database for the overturning moment in the selected axis } \\
\text { will be shown in the left side. The overturning moment is plotted as a function } \\
\text { of wind speed and direction. }\end{array}$ \\
\hline Plot peak response & $\begin{array}{l}\text { Place the button of Plot peak response in the bottom left corner of the page } \\
\text { The peak response database of overturning moments in both } x \text { - and } y \text { - } \\
\text { axes will be shown along MRIs. } \\
\text { Note) Overturning moments for any MRI can be obtained from this plot. }\end{array}$ \\
\hline \multicolumn{2}{|c|}{ Demand-to-Capacity Index (DCI) } \\
\hline \multicolumn{2}{|c|}{ Once the button is clicked, the demand-to-capacity index module is activated. } \\
\hline Load case & 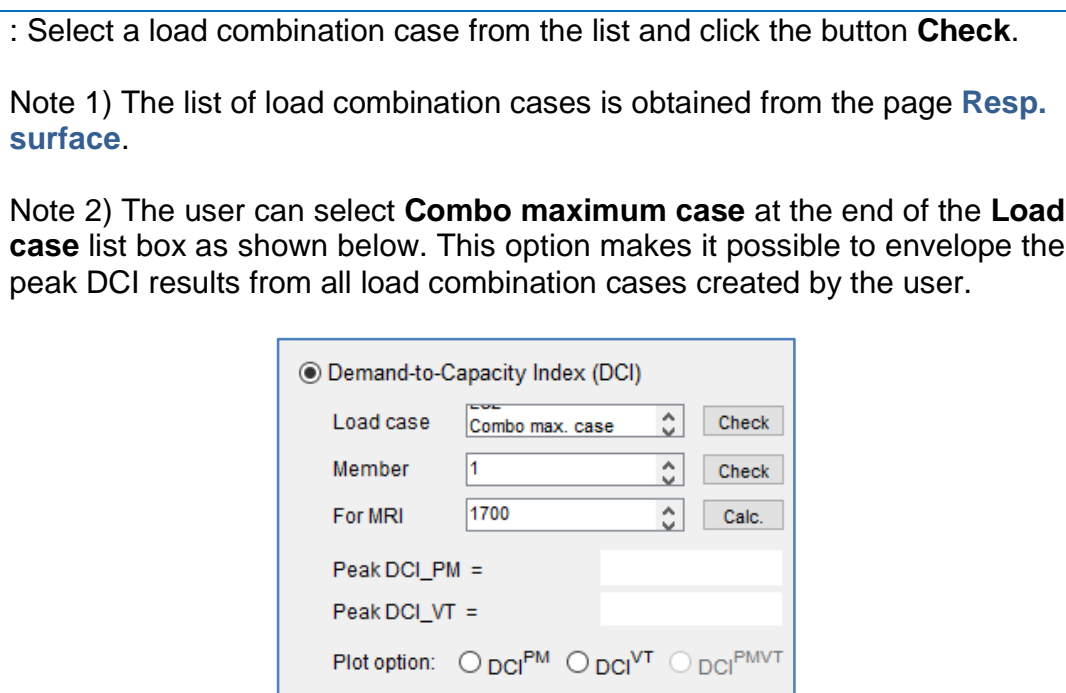 \\
\hline Member & $\begin{array}{l}\text { Select a member of interest from the list of members and click the button of } \\
\text { Check } \\
\text { Note) The list of members is obtained from the page Response DB. }\end{array}$ \\
\hline For MRI & $\begin{array}{l}\text { : Select a MRI of interest from the list of MRIs, and click the button of Calc. } \\
\text { will be shown in the blanks. } \\
\text { Note 1) The list of MRIs for the demand-to-capacity index is obtained from } \\
\text { MRIs for demand-to-capacity index in the page Wind Effects. } \\
\text { Note 2) DCIPM denotes the DCI for axial force and moments, DCI } \\
\text { forces and torsional moments, and DCIPMVT for all the internal forces and } \\
\text { moments. } \\
\text { Note 3) If the lower limit requirement of overturning moments is used in this } \\
\text { software by clicking the option of the requirement in the section Lower limit } \\
\text { requirement of the page Wind loads, the calculated DCls are adjusted } \\
\text { accordingly. }\end{array}$ \\
\hline Plot response database & $\begin{array}{l}\text { : Place the button of Plot response database at the bottom left corner of the } \\
\text { page after a plot option }\left(\mathrm{DCl}^{\mathrm{PM}}, \mathrm{DCl} \mathrm{VT}^{\mathrm{VT}} \text {, or } \mathrm{DCl} \mathrm{PMT}^{\mathrm{MVT}}\right) \text { is chosen } \\
\text { The response database for the } \mathrm{DCl} \text { of the selected member will be shown } \\
\text { in the left side. The index is plotted as a function of wind speed and direction. }\end{array}$ \\
\hline
\end{tabular}




\begin{tabular}{|c|c|}
\hline Plot peak response & $\begin{array}{l}\text { : Place the button of Plot peak response at the bottom left corner of the page } \\
\text { after a member is selected on the member list } \\
\text { MRIs. } \\
\text { The peak response database of } \mathrm{DCl} \text { s for the member will be shown along } \\
\text { show) Peak } \mathrm{DCl} \text { for any MRI can be obtained from this plot. The indexes } \\
\text { for overturning moments is not selected. }\end{array}$ \\
\hline \multicolumn{2}{|l|}{ Inter-story drift ratio } \\
\hline \multicolumn{2}{|c|}{ Once the button is clicked, the inter-story drift ratio module is activated. } \\
\hline Column set & $\begin{array}{l}\text { : Select a column line set of interest from the list of column line sets, and click } \\
\text { the button Check } \\
\text { Note) The list of column line sets is obtained from the page Wind Effects. }\end{array}$ \\
\hline Story & $\begin{array}{l}\text { : Select a story of interest from the list of all stories of the structure, and click } \\
\text { the button Check } \\
\text { Note) The list of stories in the structure is obtained from No. of stories in the } \\
\text { page Bldg. modeling. }\end{array}$ \\
\hline For MRI & $\begin{array}{l}\text { Select a MRI of interest from the list of MRIs, and click the button Calc. } \\
\text { selected column line set for the selected MRI will be shown in the blanks. } \\
\text { Note) The list of MRIs for inter-story drift is obtained from the page Wind } \\
\text { Effects. }\end{array}$ \\
\hline Plot response database & $\begin{array}{l}\text { Place the button Plot response database at the bottom left corner of the } \\
\text { page after a plot option ( } X \text {-axis or } Y \text {-axis) is chosen } \\
\text { The response database for inter-story drift ratio in the selected direction } \\
\text { will be shown in the left side. The inter-story drift ratio is plotted as a function } \\
\text { of wind speed and direction. }\end{array}$ \\
\hline Plot peak response & $\begin{array}{l}\text { Place the button Plot peak response at the bottom left corner of the page } \\
\text { The peak response database of inter-story drift ratios in both } X \text { - and } Y \text { - } \\
\text { directions will be shown along MRIs. } \\
\text { Note: Peak inter-story drift ratio for any MRI can be obtained from this plot. }\end{array}$ \\
\hline \multicolumn{2}{|l|}{ Acceleration } \\
\hline \multicolumn{2}{|c|}{ Once the button is clicked, the top floor acceleration module is activated. } \\
\hline Column set & $\begin{array}{l}\text { : Select a column line set of interest from the list of column line sets, and click } \\
\text { the button Check } \\
\text { Note) The list of column line sets is obtained from the page Wind Effects. }\end{array}$ \\
\hline Floor & $\begin{array}{l}\text { : Select a floor (story) of interest from the list of all floors of the structure, and } \\
\text { click the button Check } \\
\text { Note) The list of floors in the structure is obtained from No. of stories in the } \\
\text { page Bldg. modeling. }\end{array}$ \\
\hline
\end{tabular}




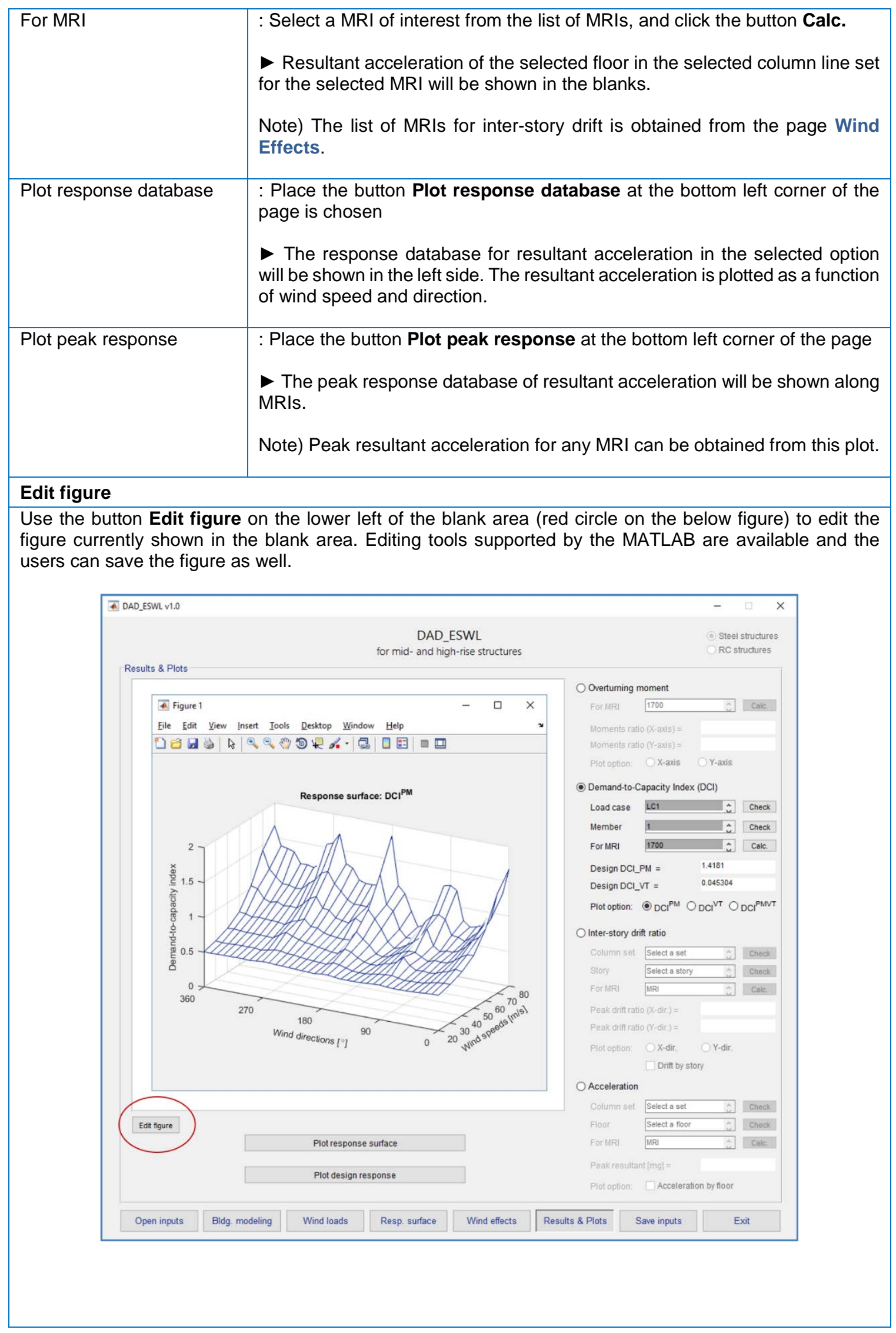




\section{Appendix II. Time integration of modal equations via ramp-invariant filters ${ }^{1}$}

\section{A2.1. Introduction}

Let $H(s)=X(s) / F(s)$ denote the transfer function of a continuous-time system, where $F(s)$ and $X(s)$ denote the Laplace transforms of the input $f(t)$ and the response $x(t)$, respectively. Then, as shown by Sterns (1975), if the input is given by discrete samples in time $f_{m}=f\left(t_{m}\right)$ and is assumed to vary linearly between these time samples, then the exact values of the response $x_{m}=x\left(t_{m}\right)$ at these time values can be obtained from a recursive filter in the following form:

$$
x_{m}=\sum_{n=0}^{M} b_{n} f_{m-n}-\sum_{n=1}^{M} a_{n} x_{m-n}
$$

where the filter coefficients appear in the numerator and denominator of the discrete-time transfer function $\tilde{H}(z)$ :

$$
\tilde{H}(z)=\frac{b_{0}+b_{1} z^{-1}+\cdots+b_{M} z^{-M}}{b_{0}+a_{1} z^{-1}+\cdots+a_{M} z^{-M}}
$$

The discrete-time transfer function $\tilde{H}(z)$ that gives an exact simulation for piecewise linear input (denoted a "ramp invariant simulation" by Stearns) is obtained from the corresponding continuous-time transfer function $X(s)$ as follows:

$$
\tilde{H}(z)=\frac{(z-1)^{2}}{T z} Z\left\{L^{-1}\left[\frac{H(s)}{s^{2}}\right]\right\}
$$

where $L^{-1}[]$ denotes the inverse Laplace transform and $Z\{\}$ denotes the $z$-transform (the discrete-time analogue of the Laplace transform).

Using this approach, Smallwood (1980) presented filter coefficients for a single degree of freedom oscillator subjected to a prescribed displacement or acceleration base input. In the following, the same approach is used to obtain filter coefficients to evaluate the response to generalized forces in modal coordinates. Additional details in the evaluation of $\tilde{H}(z)$ are included that were omitted by Smallwood for brevity. Separate filter coefficients are obtained for evaluation of the modal displacements and velocities, and the accelerations can then be obtained from these using the equation of motion.

\footnotetext{
${ }^{1}$ Written by Dr. Joseph A. Main of National Institute of Standard and Technology
} 


\section{A2.2. Modal equations of motion}

The equations of motion for a linear multi-degree of freedom system can be expressed as follows:

$$
\mathbf{M} \ddot{\mathbf{q}}+\mathbf{C} \dot{\mathbf{q}}+\mathbf{K q}=\mathbf{p}(t)
$$

Assuming classical damping, the following orthogonality relations

$$
\begin{aligned}
& \mathbf{x}_{j}^{T} \mathbf{M} \mathbf{x}_{k}=\mu_{k} \delta_{k j} ; \mathbf{x}_{j}^{T} \mathbf{K} \mathbf{x}_{k}=\mu_{k} \omega_{0 k}^{2} \delta_{k j} \\
& \mathbf{x}_{j}^{T} \mathbf{C} \mathbf{x}_{k}=2 \zeta_{k} \omega_{0 k} \mu_{k} \delta_{k j} \\
& \mathbf{x}_{k}^{T} \mathbf{p}(t)=\mu_{k} \phi_{k}(t)
\end{aligned}
$$

yield the following uncoupled modal equations of motion:

$$
\ddot{\xi}_{k}+2 \zeta_{k} \omega_{0 k} \dot{\xi}_{k}+\omega_{0 k}^{2} \xi_{k}=\phi_{k}(t)
$$

Zero initial conditions will be considered.

\section{A2.3. Ramp-invariant filter for modal displacements}

Consider the equation of motion for a particular mode, omitting the mode index $k$ for conciseness:

$$
\ddot{\xi}+2 \zeta \omega_{0} \dot{\xi}+\omega_{0}^{2} \xi=\phi(t)
$$

Denote the Laplace transforms of the modal displacement and generalized force as follows:

$$
\begin{aligned}
& \mathrm{L}\{\xi\}=X(s) \\
& \mathrm{L}\{\phi\}=F(s)
\end{aligned}
$$

Using the following derivative relationships

$$
\mathrm{L}\{\dot{\xi}\}=s X(s)-\xi\left(0^{+}\right) ; \mathrm{L}\{\ddot{\xi}\}=s^{2} X(s)-s \xi\left(0^{+}\right)-\dot{\xi}\left(0^{+}\right)
$$

the Laplace transforms of Eq. A2.9 is given by

$$
s^{2} X(s)-s \xi_{0}-\dot{\xi}_{0}+2 \zeta \omega\left[s X(s)-\xi_{0}\right]+\omega^{2} X(s)=F(s)
$$


which can be regrouped as

$$
\left[s^{2}+2 \zeta \omega s+\omega^{2}\right] X(s)-[s+2 \zeta \omega] \xi_{0}-\dot{\xi}_{0}=F(s)
$$

The Laplace transform of the response can then be expressed as:

$$
X(s)=\frac{F(s)+\left(s+2 \zeta \omega_{0}\right) \xi_{0}+\dot{\xi}_{0}}{s^{2}+2 \zeta \omega_{0} s+\omega_{0}^{2}}
$$

Assuming zero initial conditions, the continuous-time transfer function is given by:

$$
H(s)=\frac{X(s)}{F(s)}=\frac{1}{s^{2}+2 \zeta \omega_{0} s+\omega_{0}^{2}}
$$

Combining Eq. A2.16 and Eq. A2.3, the discrete-time transfer function for the rampinvariant simulation for the modal displacements is given by:

$$
\tilde{H}(z)=\frac{(z-1)^{2}}{T z} Z\left\{L^{-1}\left[\frac{1}{s^{2}\left(s^{2}+2 \zeta \omega_{0} s+\omega_{0}^{2}\right)}\right]\right\}
$$

Evaluate the inverse Laplace Transform using the convolution theorem:

$$
\begin{aligned}
& \mathrm{L}^{-1}\left\{\frac{1}{s^{2}}\right\}=t \\
& \mathrm{~L}^{-1}\left\{\frac{1}{s^{2}+2 \zeta \omega_{0} s+\omega_{0}^{2}}\right\}=\frac{1}{\omega} e^{-\zeta \omega_{0} t} \sin \omega t
\end{aligned}
$$

where $\omega=\omega_{0} \sqrt{1-\zeta^{2}}$ is the damped frequency, from which it follows that $\omega^{2}=\omega_{0}^{2}-\omega_{0}^{2} \zeta^{2}$. Using the convolution theorem, it then follows that

$$
\begin{array}{r}
L^{-1}\left\{\frac{1}{s^{2}} \frac{1}{s^{2}+2 \zeta \omega_{0} s+\omega_{0}^{2}}\right\}=\frac{1}{\omega} \int_{0}^{t}(t-\tau) e^{-\zeta \omega_{0} \tau} \sin \omega \tau d \tau \\
\quad=\frac{\omega_{0} t-2 \zeta}{\omega_{0}^{3}}+\frac{e^{-\zeta \omega_{0} t}}{\omega_{0}^{3}}\left[2 \zeta \cos \omega t-\frac{1-2 \zeta^{2}}{\sqrt{1-\zeta^{2}}} \sin \omega t\right]
\end{array}
$$

In which the following identity is noted:

$$
\frac{\omega_{0}^{2}-2 \omega^{2}}{\omega \omega_{0}}=\frac{\omega_{0}^{2}-2 \omega_{0}^{2}\left(1-\zeta^{2}\right)}{\omega_{0}^{2} \sqrt{1-\zeta^{2}}}=-\frac{1-2 \zeta^{2}}{\sqrt{1-\zeta^{2}}}
$$


The discrete-time transfer function can then be expressed as

$$
\tilde{H}(z)=\frac{(z-1)^{2}}{T z} Z\left\{\frac{\omega_{0} t-2 \zeta}{\omega_{0}^{3}}+\frac{e^{-\zeta \omega_{0} t}}{\omega_{0}^{3}}\left[2 \zeta \cos \omega t-\frac{1-2 \zeta^{2}}{\sqrt{1-\zeta^{2}}} \sin \omega t\right]\right\}
$$

The $z$-transforms of each term in Eq. A2.22 are evaluated as

$$
\begin{aligned}
& \mathrm{Z}\left\{\frac{\omega_{0} t-2 \zeta}{\omega_{0}^{3}}\right\}=\frac{T z}{\omega_{0}^{2}(z-1)^{2}}-\frac{2 \zeta z}{\omega_{0}^{3}(z-1)} \\
& Z\left\{\frac{2 \zeta e^{-\zeta \omega_{0} t}}{\omega_{0}^{3}} \cos \omega t\right\}=\frac{2 \zeta}{\omega_{0}^{3}} \frac{z^{2}-z e^{-\zeta \omega_{0} T} \cos \omega T}{z^{2}-2 z e^{-\zeta \omega_{0} T} \cos \omega T+e^{-2 \zeta \omega_{0} T}} \\
& Z\left\{-\frac{e^{-\zeta \omega_{0} t}}{\omega_{0}^{3}} \frac{1-2 \zeta^{2}}{\sqrt{1-\zeta^{2}}} \sin \omega t\right\}=-\frac{1}{\omega_{0}^{3}} \frac{1-2 \zeta^{2}}{\sqrt{1-\zeta^{2}}} \frac{z e^{-\zeta \omega_{0} T} \sin \omega T}{z^{2}-2 z e^{-\zeta \omega_{0} T} \cos \omega T+e^{-2 \zeta \omega_{0} T}}
\end{aligned}
$$

Combining these terms with Eq. A2.22, the discrete-time transfer function can then be expressed as

$$
\tilde{H}(z)=\frac{b_{0}+b_{1} z^{-1}+b_{2} z^{-2}}{1+a_{1} z^{-1}+a_{2} z^{-2}}
$$

where the filter coefficients are given by:

$$
\begin{aligned}
& a_{1}=-2 C \\
& a_{2}=E^{2} \\
& b_{0}=\frac{1}{T \omega_{0}^{3}}\left[2 \zeta(C-1)-\frac{1-2 \zeta^{2}}{\sqrt{1-\zeta^{2}}} S+T \omega_{0}\right] \\
& b_{1}=\frac{1}{T \omega_{0}^{3}}\left[-2 C T \omega_{0}+2 \zeta\left(1-E^{2}\right)+\frac{2\left(1-2 \zeta^{2}\right)}{\sqrt{1-\zeta^{2}}} S\right] \\
& b_{2}=\frac{1}{T \omega_{0}^{3}}\left[E^{2}\left(T \omega_{0}+2 \zeta\right)-2 \zeta C-\frac{1-2 \zeta^{2}}{\sqrt{1-\zeta^{2}}} S\right]
\end{aligned}
$$

in which the following symbols are introduced for convenience, following Smallwood (1980): 


$$
E=e^{-\zeta \omega_{0} T}, C=E \cos \omega T, S=E \sin \omega T
$$

Multiplying the filter coefficients $b_{m}$ in Eqs. A2.29 to A2.31 by $\omega_{0}^{2}$ yields expressions that are equivalent to those obtained by Smallwood (1980) for the "relative displacement model”. The modal displacements are then evaluated using the following recursive filter:

$$
\xi_{m}=\sum_{n=0}^{2} b_{n} \phi_{m-n}-\sum_{n=1}^{2} a_{n} \xi_{m-n}
$$

\section{A2.4. Ramp-invariant filter for modal velocities}

The modal velocities can be evaluated starting with the following continuous-time transfer function:

$$
H_{v}(s)=\frac{s X(s)}{F(s)}=\frac{s}{s^{2}+2 \zeta \omega_{0} s+\omega_{0}^{2}}
$$

The ramp-invariant simulation for modal velocities is then given by

$$
\tilde{H}_{v}(z)=\frac{(z-1)^{2}}{T z} Z\left\{L^{-1}\left[\frac{s}{s\left(s^{2}+2 \zeta \omega_{0} s+\omega_{0}^{2}\right)}\right]\right\}
$$

It is noted that Smallwood (1980) obtained the following result for the "absolute acceleration” model:

$$
\tilde{H}_{a a}(z)=\frac{(z-1)^{2}}{T z} Z\left\{L^{-1}\left[\frac{2 \zeta \omega_{0} s+\omega_{0}^{2}}{s\left(s^{2}+2 \zeta \omega_{0} s+\omega_{0}^{2}\right)}\right]\right\}=\frac{b_{a a 0}+b_{a a 1} z^{-1}+b_{a a 2} z^{-2}}{1+a_{1} z^{-1}+a_{2} z^{-2}}
$$

where $a_{1}$ and $a_{2}$ are given in Eqs. (2.27 and A2.28, and the filter coefficients in the numerator are given by

$$
\begin{aligned}
& b_{a a 0}=1-S^{\prime} \\
& b_{a a 1}=2\left(S^{\prime}-C\right) \\
& b_{a a 2}=E^{2}-S^{\prime}
\end{aligned}
$$

in which $\mathrm{E}$ and $\mathrm{C}$ are given by Eq. A2.32, and the following symbol is introduced:

$$
S^{\prime}=\frac{E \sin \omega T}{\omega T}
$$


The following relationship was derived previously (Eqs. A2.17 and A2.26):

$$
\tilde{H}(z)=\frac{(z-1)^{2}}{T z} Z\left\{L^{-1}\left[\frac{1}{s^{2}\left(s^{2}+2 \zeta \omega_{0} s+\omega_{0}^{2}\right)}\right]\right\}=\frac{b_{0}+b_{1} z^{-1}+b_{2} z^{-2}}{1+a_{1} z^{-1}+a_{2} z^{-2}}
$$

Combining Eqs. (A36) and (A41), it follows from the linearity of the system that $\tilde{H}_{v}(z)$ in Eq. (A35) can be expressed as

$$
\tilde{H}_{v}(z)=\frac{\tilde{H}_{a a}(z)-\omega_{0}^{2} \tilde{H}(z)}{2 \zeta \omega_{0}}
$$

The discrete time transfer function for the velocities can then be expressed as

$$
\tilde{H}_{v}(z)=\frac{b_{v 0}+b_{v 1} z^{-1}+b_{v 2} z^{-2}}{1+a_{1} z^{-1}+a_{2} z^{-2}}
$$

where $a_{1}$ and $a_{2}$ are given in Eqs. A2.27 and A2.28, and the filter coefficients in the numerator are given by

$$
\begin{aligned}
& b_{v 0}=\frac{b_{a a 0}-\omega_{0}^{2} b_{0}}{2 \zeta \omega_{0}}=\frac{1}{2 T \zeta \omega_{0}^{2}}\left[-T \omega_{0} S^{\prime}+2 \zeta(1-C)+\frac{\left(1-2 \zeta^{2}\right) S}{\sqrt{1-\zeta^{2}}}\right] \\
& b_{v 1}=\frac{b_{a a 1}-\omega_{0}^{2} b_{1}}{2 \zeta \omega_{0}}=\frac{1}{2 T \zeta \omega_{0}^{2}}\left[2 T \omega_{0} S^{\prime}-2 \zeta\left(1-E^{2}\right)-\frac{2\left(1-2 \zeta^{2}\right) S}{\sqrt{1-\zeta^{2}}}\right] \\
& b_{v 2}=\frac{b_{a a 2}-\omega_{0}^{2} b_{2}}{2 \zeta \omega_{0}}=\frac{1}{2 T \zeta \omega_{0}^{2}}\left[-T \omega_{0} S^{\prime}-2 \zeta E^{2}+2 \zeta C+\frac{\left(1-2 \zeta^{2}\right) S}{\sqrt{1-\zeta^{2}}}\right]
\end{aligned}
$$

The modal velocities are then evaluated using the following recursive filter:

$$
\dot{\xi}_{m}=\sum_{n=0}^{2} b_{v n} \phi_{m-n}-\sum_{n=1}^{2} a_{n} \dot{\xi}_{m-n}
$$

The modal accelerations do not need to be separately evaluated using a filter, but can be obtained from the modal displacements and velocities and the generalized force using the equation of motion (Eq. A2.9):

$$
\ddot{\xi}_{m}=\phi\left(t_{m}\right)-2 \zeta \omega_{0} \dot{\xi}_{m}-\omega_{0}^{2} \xi_{m}
$$




\section{A2.5. Verification of the solver}

This section validates the proper functioning of the solver of the DAD_ESWL software using the ramp-invariant filter mentioned in this Appendix. Two simple single- and multidegree-of-freedom (SDOF and MDOF, respectively) examples will be solved by the solver and compared to the closed form solutions. The examples represent simple 2D 1-story and 2-story buildings, assumed to be linearly elastic and have negligible the second-order effects. The closed-form solutions and derivations of the examples can be found in Sect. 3.2.7 for SDOF example, Sect. 12.7 and Example 12.5 for MDOF example, respectively, of Chopra (2016).

\section{A2.5.1 Single-degree-of-freedom system}

A simple application designed to provide a simple validation of the solver considers a single-degree-of-freedom 1-story building excited by a sinusoidal force. Figure A2.1 shows the 1-story SDOF building model and the requisite parameters.

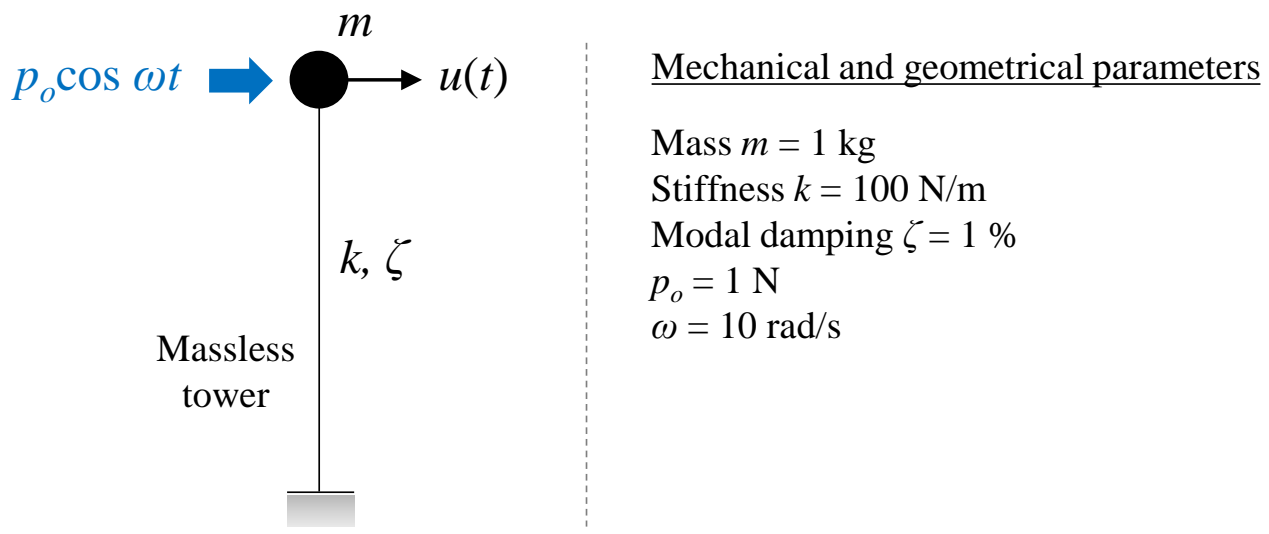

Figure A2.1. 1-story SDOF building model and assumed variables

\section{A2.5.1.1 Closed-form solution}

The differential equation governing the response of the system to harmonic force is

$$
m \ddot{u}(t)+c \dot{u}(t)+k u(t)=p_{0} \cos \omega t
$$

A particular solution (steady-state response) of this equation of motion is

$$
u(t)=C \sin \omega t+D \cos \omega t
$$

with

$$
C=\frac{p_{o}}{k} \frac{2 \zeta\left(\omega / \omega_{n}\right)}{\left[1-\left(\omega / \omega_{n}\right)^{2}\right]^{2}+\left[2 \zeta\left(\omega / \omega_{n}\right)\right]^{2}}
$$




$$
D=\frac{p_{o}}{k} \frac{1-\left(\omega / \omega_{n}\right)^{2}}{\left[1-\left(\omega / \omega_{n}\right)^{2}\right]^{2}+\left[2 \zeta\left(\omega / \omega_{n}\right)\right]^{2}}
$$

where $\omega_{n}$ is the natural frequency of vibration of the structure $(=\sqrt{\mathrm{k} / \mathrm{m}}=10 \mathrm{rad} / \mathrm{s})$. Substituting Eqs. A2.51 and A2.52 in Eq. A2.50 gives

$$
u(t)=\frac{p_{o}}{2 k \zeta} \sin \omega t
$$

Equation A2.53 is the complete solution of Eq. A2.49 for the steady-state response of the building. Substituting $p_{0}=1 \mathrm{~N}, k=100 \mathrm{~N} / \mathrm{m}, \zeta=0.01$, and $\omega=10 \mathrm{rad} / \mathrm{s}$ in Eq. A2.53 yields the time series of displacement of this SDOF example as

$$
u(t)=\frac{1}{2} \sin 10 t
$$

\section{A2.5.1.2 Numerical solution}

For this example, the MATLAB functions performing a role of the solver embedded in the DAD_ESWL software (a.k.a 'F_and_M_gen.m' and 'modal_eom_solve.m') were used separately. The input variables for the example are summarized in Table A2.1. The forcing function $p_{o} \cos \omega t$ is described considering a sampling frequency of $100 \mathrm{~Hz}$ for a duration time of $300 \mathrm{~s}$.

The analysis is performed by running the function 'F_and_M_gen.m' which execute the solver function 'modal_eom_solve.m'. The generalized displacements (variable 'gen_d') is obtained through those functions. The actual displacements can be obtained thereafter through multiplication by the eigen vector (variable 'evectors', which is 1 in this example). The result will be presented in the next section with that from the closed-form solution together.

Table A2.1. Summary of input variables for SDOF building example

\begin{tabular}{lll}
\hline Structural properties & & \\
\hline MATLAB variable & Value & Description \\
(see User's Manual for details) & $1[\mathrm{~kg}]$ & Mass \\
mass & 1 & Mode shape \\
evectors & $1[\%]$ & Damping ratio \\
$D \_s t r$ & $0.628[\mathrm{~s}]$ & Natural period of vibration \\
$T$ _str & & \\
\hline Sinusoidal excitation & & \\
\hline MATLAB variable & Value & Description \\
(see User's Manual for details) & & \\
freq & $100[\mathrm{~Hz}]$ & Sampling frequency \\
Npoints & 30000 & Number of sampling points \\
\hline
\end{tabular}




\section{A2.5.1.3 Comparison between closed-form and numerical solutions}

Figures A2.2 shows the comparison between the time series of the displacements $u(t)$ obtained in the closed-form solution from Eq. A2.54, with those estimated by running the DAD_ESWL solver. As can be seen the numerical results are in good agreement with the exact solution. The maximum displacement obtained in the closed-form and in the numerical analysis are $0.5 \mathrm{~m}$ and $0.4996 \mathrm{~m}$, respectively. A good agreement can be seen in all cases with any differences due to the numerical integration.

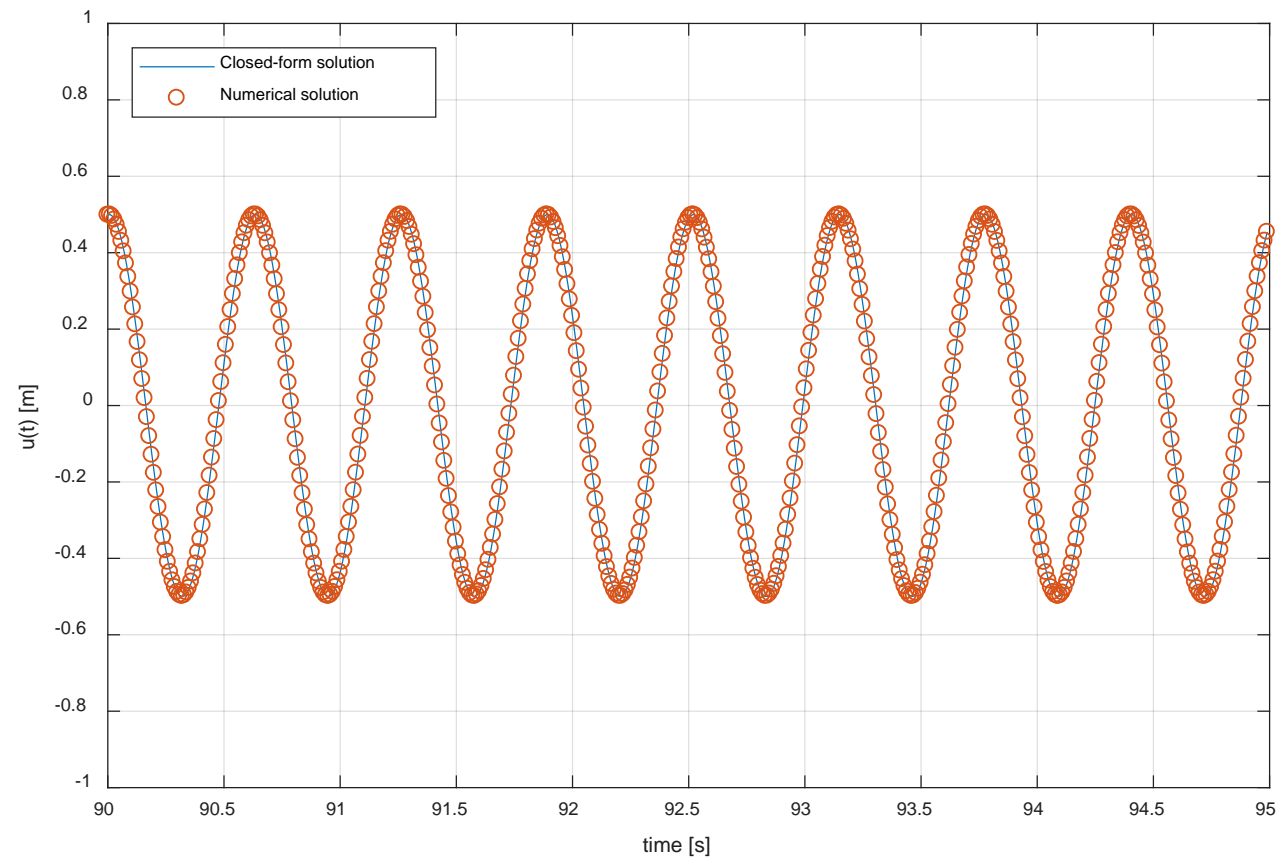

Figure A2.2. Comparison of closed-form and numerical analysis time series of $u$ 


\section{A2.5.2 Multi-degree-of-freedom system}

A simple application designed to provide a simple validation of the solver considers a multi-degree-of-freedom 2-story building excited by a sinusoidal force applied on the first floor. Figure A2.3 shows the 2-story MDOF building model and the requisite parameters.

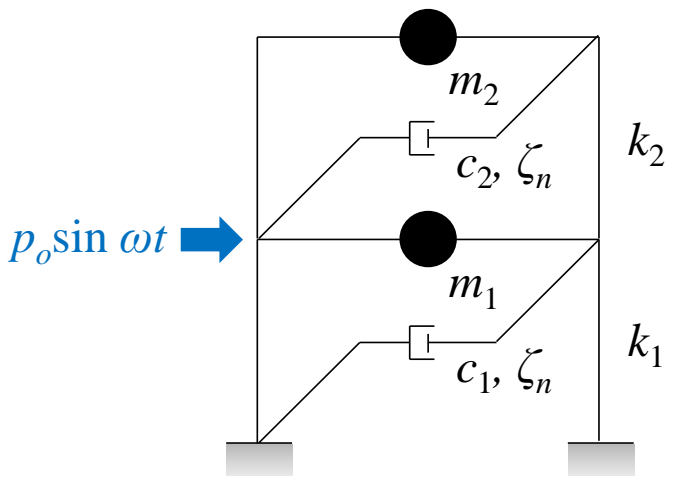

\section{Mechanical and geometrical parameters}

Mass $m=1 \mathrm{~kg}$

Stiffness $k=100 \mathrm{~N} / \mathrm{m}$

Modal damping $\zeta_{n}=1 \%$ for $n=11^{\text {st }}, 2^{\text {nd }}$ mode

$p_{o}=1 \mathrm{~N}$

$\left(\mathrm{c}=2 \zeta_{n} \omega_{n} m\right)$

$\omega=10 \mathrm{rad} / \mathrm{s}$

$m_{1}=2 m \quad k_{1}=2 k \quad c_{1}=c$

$m_{2}=m \quad k_{2}=k \quad c_{2}=c$

Figure A2.3. 2-story MDOF building model and assumed variables

\section{A2.5.2.1 Closed-form solution}

From the information given in Fig. A2.3, the mass and stiffness matrices ( $\mathbf{m}$ and $\mathbf{k}$, respectively) of the system can be obtained as

$$
\begin{aligned}
& \mathbf{m}=\left[\begin{array}{cc}
2 m & 0 \\
0 & m
\end{array}\right] \\
& \mathbf{k}=\left[\begin{array}{cc}
k_{1}+k_{2} & -k_{2} \\
-k_{2} & k_{2}
\end{array}\right]=\left[\begin{array}{cc}
3 k & -k \\
-k & k
\end{array}\right] \\
& \mathbf{c}=\left[\begin{array}{cc}
c_{1}+c_{2} & -c_{2} \\
-c_{2} & c_{2}
\end{array}\right]=\left[\begin{array}{cc}
3 c & -c \\
-c & c
\end{array}\right]
\end{aligned}
$$

The frequency equation is $\operatorname{det}\left[\mathbf{k}-\omega_{n}^{2} \mathbf{m}\right]=0$, which, after substituting for $\mathbf{m}$ and $\mathbf{k}$ and evaluating the determinant, can be written as

$$
\left(2 m^{2}\right) \omega^{4}+(-5 k m) \omega^{2}+2 k^{2}=0
$$

The two roots are $\omega_{1}^{2}=k / 2 m$ and $\omega_{2}^{2}=2 k / m$, and the two natural frequencies are

$$
\omega_{1}=\sqrt{\frac{k}{2 m}} \text { and } \omega_{2}=\sqrt{\frac{2 k}{m}}
$$


The natural modes (eigen vectors) determined from $\left[\mathbf{k}-\omega_{n}^{2} \mathbf{m}\right] \phi_{n}=0$ are

$$
\phi_{1}=\left[\begin{array}{c}
1 / 2 \\
1
\end{array}\right] \text { and } \phi_{2}=\left[\begin{array}{c}
-1 \\
1
\end{array}\right]
$$

By using the modal orthogonality, the generalized mass $\left(M_{n}\right)$, stiffness $\left(K_{n}\right)$, and the sinusoidal excitation $\left(P_{n}\right)$ for each mode $n=1$ and 2 are

$$
\begin{aligned}
& M_{1}=\phi_{1}^{T} \mathbf{m} \phi_{1}=\left[\begin{array}{ll}
\frac{1}{2} & 1
\end{array}\right]\left[\begin{array}{cc}
2 m & 0 \\
0 & m
\end{array}\right]\left[\begin{array}{c}
\frac{1}{2} \\
1
\end{array}\right]=\frac{3}{2} m \\
& M_{2}=\phi_{2}^{T} \mathbf{m} \phi_{2}=\left[\begin{array}{ll}
-1 & 1
\end{array}\right]\left[\begin{array}{cc}
2 m & 0 \\
0 & m
\end{array}\right]\left[\begin{array}{c}
-1 \\
1
\end{array}\right]=3 m \\
& K_{1}=\phi_{1}^{T} \mathbf{k} \phi_{1}=\left[\begin{array}{cc}
\frac{1}{2} & 1
\end{array}\right]\left[\begin{array}{cc}
3 k & -k \\
-k & k
\end{array}\right]\left[\begin{array}{c}
\frac{1}{2} \\
1
\end{array}\right]=\frac{3}{4} k \\
& K_{2}=\phi_{2}^{T} \mathbf{k} \phi_{2}=\left[\begin{array}{ll}
-1 & 1
\end{array}\right]\left[\begin{array}{cc}
3 k & -k \\
-k & k
\end{array}\right]\left[\begin{array}{c}
-1 \\
1
\end{array}\right]=6 k \\
& C_{1}=\phi_{1}^{T} \mathbf{c} \phi_{1}=\left[\begin{array}{cc}
\frac{1}{2} & 1
\end{array}\right]\left[\begin{array}{cc}
3 c & -c \\
-c & c
\end{array}\right]\left[\begin{array}{c}
\frac{1}{2} \\
1
\end{array}\right]=\frac{3}{4} c \\
& C_{2}=\phi_{2}^{T} \mathbf{c} \phi_{2}=\left[\begin{array}{ll}
-1 & 1
\end{array}\right]\left[\begin{array}{cc}
3 c & -c \\
-c & c
\end{array}\right]\left[\begin{array}{c}
-1 \\
1
\end{array}\right]=6 c \\
& P_{1}(t)=\phi_{1}^{T} \mathbf{p}(t)=\left[\begin{array}{ll}
\frac{1}{2} & 1
\end{array}\right]\left[\begin{array}{cc}
p_{o} \sin \omega t & 0 \\
0 & 0
\end{array}\right]=\frac{p_{o}}{2} \sin \omega t \\
& P_{2}(t)=\phi_{2}^{T} \mathbf{p}(t)=\left[\begin{array}{ll}
-1 & 1
\end{array}\right]\left[\begin{array}{cc}
p_{o} \sin \omega t & 0 \\
0 & 0
\end{array}\right]=-p_{o} \sin \omega t
\end{aligned}
$$

The modal equation of motion for the system becomes

$$
M_{n} \ddot{q}_{n}(t)+C_{n} \dot{q}_{n}(t)+K_{n} q_{n}(t)=P_{n} \sin \omega t
$$

To solve Eq. A2.69, let us draw upon the solution presented in Eq. A2.50 for an SDOF system subjected to harmonic force. The governing equation is 


$$
m \ddot{u}(t)+c \dot{u}(t)+k u(t)=p_{0} \sin \omega t
$$

and its steady-state solution is

$$
u(t)=\frac{p_{o}}{k}(C \sin \omega t+D \cos \omega t)
$$

with

$$
\begin{aligned}
& C=\frac{1-\left(\omega / \omega_{n}\right)^{2}}{\left[1-\left(\omega / \omega_{n}\right)^{2}\right]^{2}+\left[2 \zeta\left(\omega / \omega_{n}\right)\right]^{2}} \\
& D=\frac{-2 \zeta\left(\omega / \omega_{n}\right)}{\left[1-\left(\omega / \omega_{n}\right)^{2}\right]^{2}+\left[2 \zeta\left(\omega / \omega_{n}\right)\right]^{2}}
\end{aligned}
$$

The solution for Eq. A2.66 can be inferred from Eq. A2.68, and is

$$
q_{n}(t)=\frac{P_{n o}}{K_{n}}\left(C_{n} \sin \omega t+D_{n} \cos \omega t\right)
$$

where $C_{n}$ and $D_{n}$ are given by Eq. A2.72 to A2.73 with $\omega_{n}$, the natural frequency for the $n^{\text {th }}$ mode ( $n=1$ and 2 in this example). Substituting for $P_{n o}$ and $K_{n}$ for $n=1$ and 2 yields

$$
\begin{aligned}
& q_{1}(t)=\frac{2 p_{o}}{3 k}\left(C_{1} \sin \omega t+D_{1} \cos \omega t\right) \\
& q_{2}(t)=-\frac{p_{o}}{6 k}\left(C_{2} \sin \omega t+D_{2} \cos \omega t\right)
\end{aligned}
$$

The nodal displacements can be given by

$$
\mathbf{u}(t)=\sum_{n=1}^{N} \mathbf{u}_{n}(t)=\sum_{n=1}^{N} \phi_{n} q_{n}(t)
$$

Thus, substituting $\phi_{n}$ (Eq. A2.59) in Eq. A2.74 gives the nodal displacements as

$$
\begin{aligned}
& {\left[\begin{array}{l}
u_{1}(t) \\
u_{2}(t)
\end{array}\right]=\left[\begin{array}{cc}
\frac{1}{2} & -1 \\
1 & 1
\end{array}\right]\left[\begin{array}{l}
q_{1}(t) \\
q_{2}(t)
\end{array}\right] \text {, so }} \\
& u_{1}(t)=\frac{1}{2} q_{1}(t)-q_{2}(t) \text { and } u_{2}(t)=q_{1}(t)+q_{2}(t)
\end{aligned}
$$


Finally, substituting Eq. A2.75 to A2.76 for $q_{n}(t)$ gives

$$
\begin{aligned}
& u_{1}(t)=\frac{p_{o}}{6 k}\left[\left(2 C_{1}+C_{2}\right) \sin \omega t+\left(2 D_{1}+D_{2}\right) \cos \omega t\right] \text { and } \\
& u_{2}(t)=\frac{p_{o}}{6 k}\left[\left(4 C_{1}-C_{2}\right) \sin \omega t+\left(4 D_{1}-D_{2}\right) \cos \omega t\right]
\end{aligned}
$$

\section{A2.5.2.2 Numerical solution}

For this example, the MATLAB functions performing a role of the solver embedded in the DAD_ESWL software (a.k.a 'F_and_M_gen.m' and 'modal_eom_solve.m') were used separately. The input variables for the example are summarized in Table A2.2. The forcing function $p_{o} \sin \omega t$ is described considering a sampling frequency of $100 \mathrm{~Hz}$ for a duration time of $300 \mathrm{~s}$.

The analysis is performed by running the function ' $F \_$and_M_gen.m' which execute the solver function 'modal_eom_solve.m'. The generalized displacements (variable 'gen_d') is obtained through those functions. The actual displacements can be obtained thereafter through multiplication by the eigen vector (variable 'evectors'). The result will be presented in the next section with that from the closed-form solution together.

Table A2.2. Summary of input variables for SDOF building example

\begin{tabular}{lll}
\hline Structural properties & & \\
\hline $\begin{array}{l}\text { MATLAB variable } \\
\text { (see User's Manual for details) }\end{array}$ & Value & Description \\
mass & {$\left[\begin{array}{ll}2 & 0 \\
0 & 1\end{array}\right]$} & Mass [kg] \\
evectors & {$\left[\begin{array}{cc}0.5 & -1 \\
1 & 1\end{array}\right]$} & Mode shape \\
D_str & {$\left[\begin{array}{ll}1 & 1\end{array}\right]$} & \\
T_str & {$\left[\begin{array}{ll}0.889 & 0.444\end{array}\right]$} & Damping ratio [\%] \\
\hline Sinusoidal excitation & & Natural period of vibration [s] \\
\hline $\begin{array}{l}\text { MATLAB variable } \\
\text { (see User's Manual for details) }\end{array}$ & Value & Description \\
freq & 100 & Sampling frequency [Hz] \\
Npoints & 30000 & Number of sampling points \\
\hline
\end{tabular}




\section{A2.5.2.3 Comparison between closed-form and numerical solutions}

Figures A2.4 to A2.5 show the comparison between the time series of the displacements $u_{1}(t)$ and $u_{2}(t)$ obtained in the closed-form solution from Eq. A76, with those estimated by running the DAD_ESWL solver. As can be seen the numerical results are in good agreement with the exact solution. The maximum displacement obtained in the closedform and in the numerical analysis are 1.88E-4 m and 1.95E-4 $\mathrm{m}$ for the first floor $\left(u_{1}\right)$, respectively, and are $0.01 \mathrm{~m}$ and $0.01 \mathrm{~m}$ for the second floor $\left(u_{2}\right)$, respectively. A good agreement can be seen in all cases with any differences due to the numerical integration.

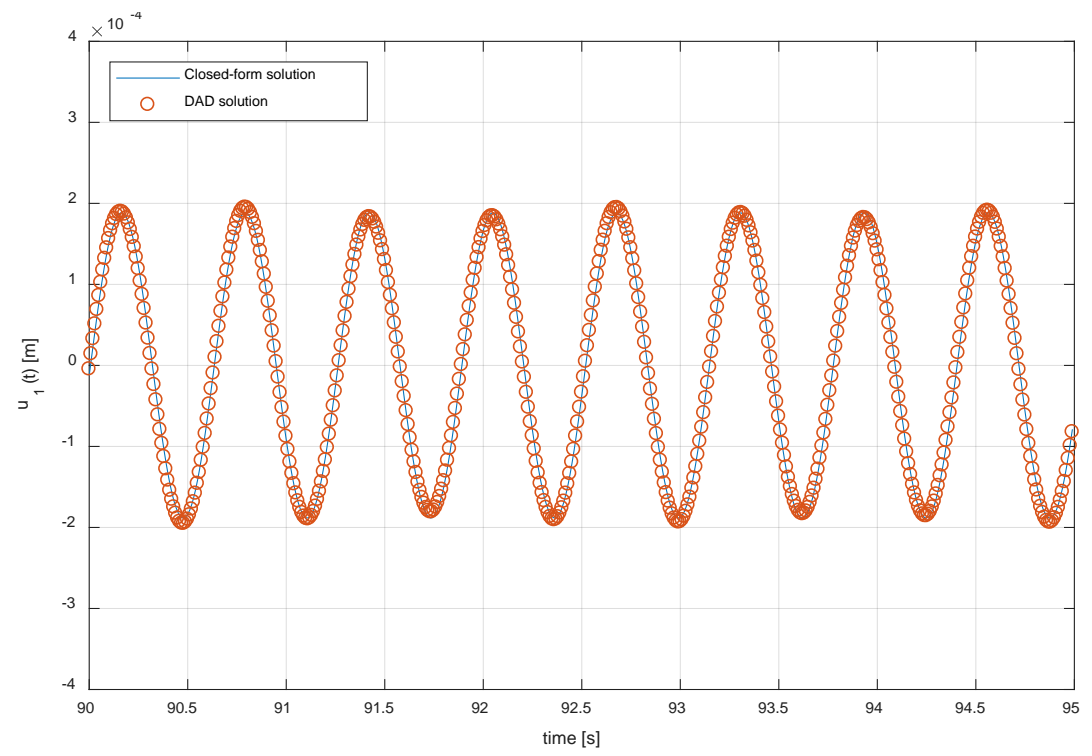

Figure A2.4. Comparison of closed-form and numerical analysis time series of $u_{1}$

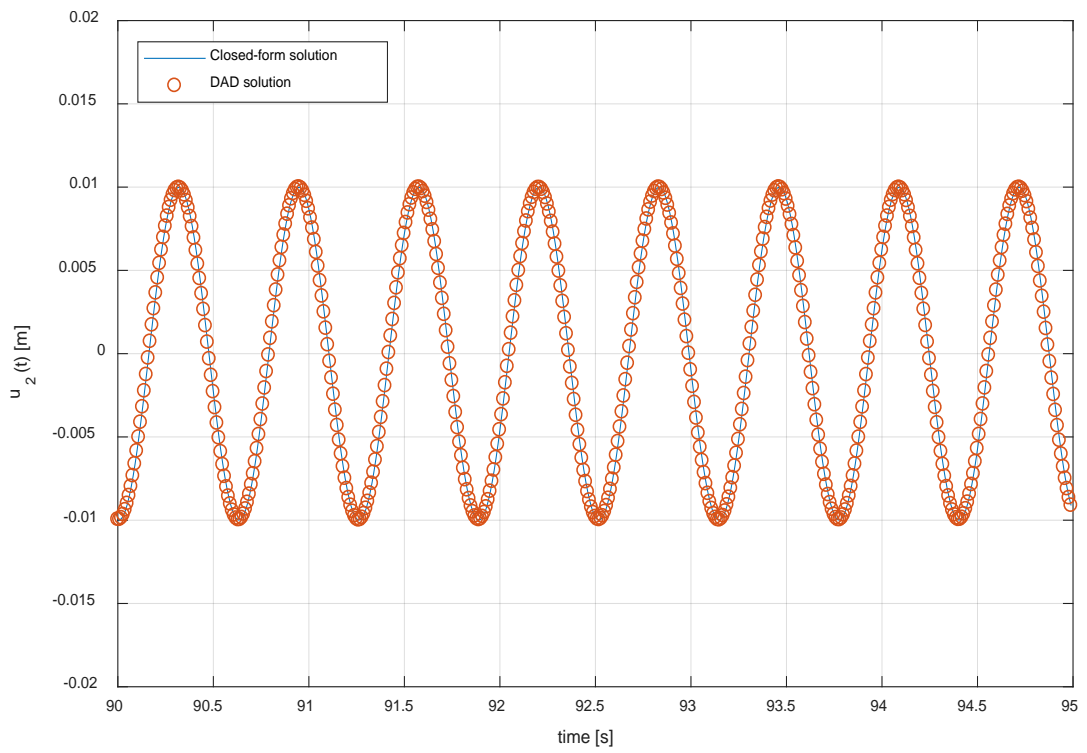

Figure A2.5. Comparison of closed-form and numerical analysis time series of $u_{2}$ 


\section{Appendix III. Verification of module for calculating bi-axial interaction surfaces}

This section verifies the module for calculating the bi-axial interaction surfaces of RC sections in the DAD_ESWL v1.0 by comparing them to those obtained in the commercial $\mathrm{RC}$ design software spColumn $\mathrm{v} 4.81^{2}$. Based on the inclined neutral axes from $\theta=0^{\circ}$ to $\theta$ $=90^{\circ}$ (see the definition of $\theta$ in Eq. 31), the factored interaction diagrams are compared. Total three example sections are used; the first two sections are columns and the last section is shear wall. The number of points used to define a two-dimensional interaction curve are 101 in the DAD_ESWL and 71 in spColumn. Note that the angles of $\theta=0^{\circ}$ and $\theta=90^{\circ}$ represent the cases where the sections are subjected to a uniaxial bending moment. In the PM diagrams with respect to each inclined neutral axis $(\theta)$, five representative points are used for validation as follows:

Point 1: Axial load strength at zero eccentricity

Point 2: Axial compressional moment at zero tensile strain

Point 3: Tensile reinforcement does not yield

Point 4: Balanced failure

Point 5: Limit of tension-controlled failure.

Each point can be expressed in the coordinates of the PM diagram where the $x$ - and $y$ coordinates correspond to the factored nominal moment $\left(\phi \mathrm{M}_{n}\right)$ and axial force $\left(\phi \mathrm{P}_{\mathrm{n}}\right)$, respectively, and the distances between origin and the selected points are used to compare the results from both programs.

\footnotetext{
2 Structurepoint, spColumn software: Design and Investigation of Reinforced Concrete Column Sections, www.structurepoint.org, accessed on 11/17/2016.
} 


\section{A3.1. Example section 1.}

Figures A3.1 and A3.2 show the example section 1 of the $800 \mathrm{~mm} \times 800 \mathrm{~mm}$ square column section with four reinforcements and the comparisons of PM interaction diagrams with respect to the angle $\theta$ from both the DAD_ESWL and the spColumn, respectively. Since the example section 1 is square and the reinforcements are placed symmetrically in all directions, the PM interaction diagrams resulted from $\theta=0^{\circ}$ to $\theta=40^{\circ}$ and $\theta=50^{\circ}$ to $\theta$ $=90^{\circ}$ are symmetrical. The results obtained from the two programs with respect to the essential five points are compared based on the distance from origin to each point. The ratios of the DAD_ESWL to the spColumn for each point are then calculated and averaged regarding each angle. It is found that the differences between the two programs are within $1 \%$ in all cases.

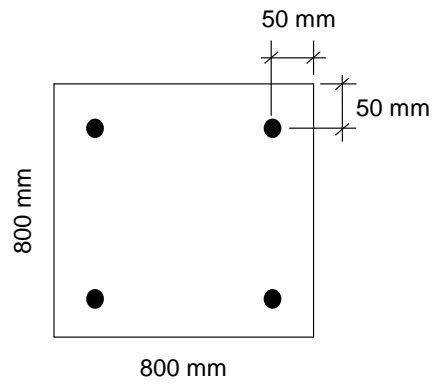

Figure A3.1. Example section 1 (Square column)
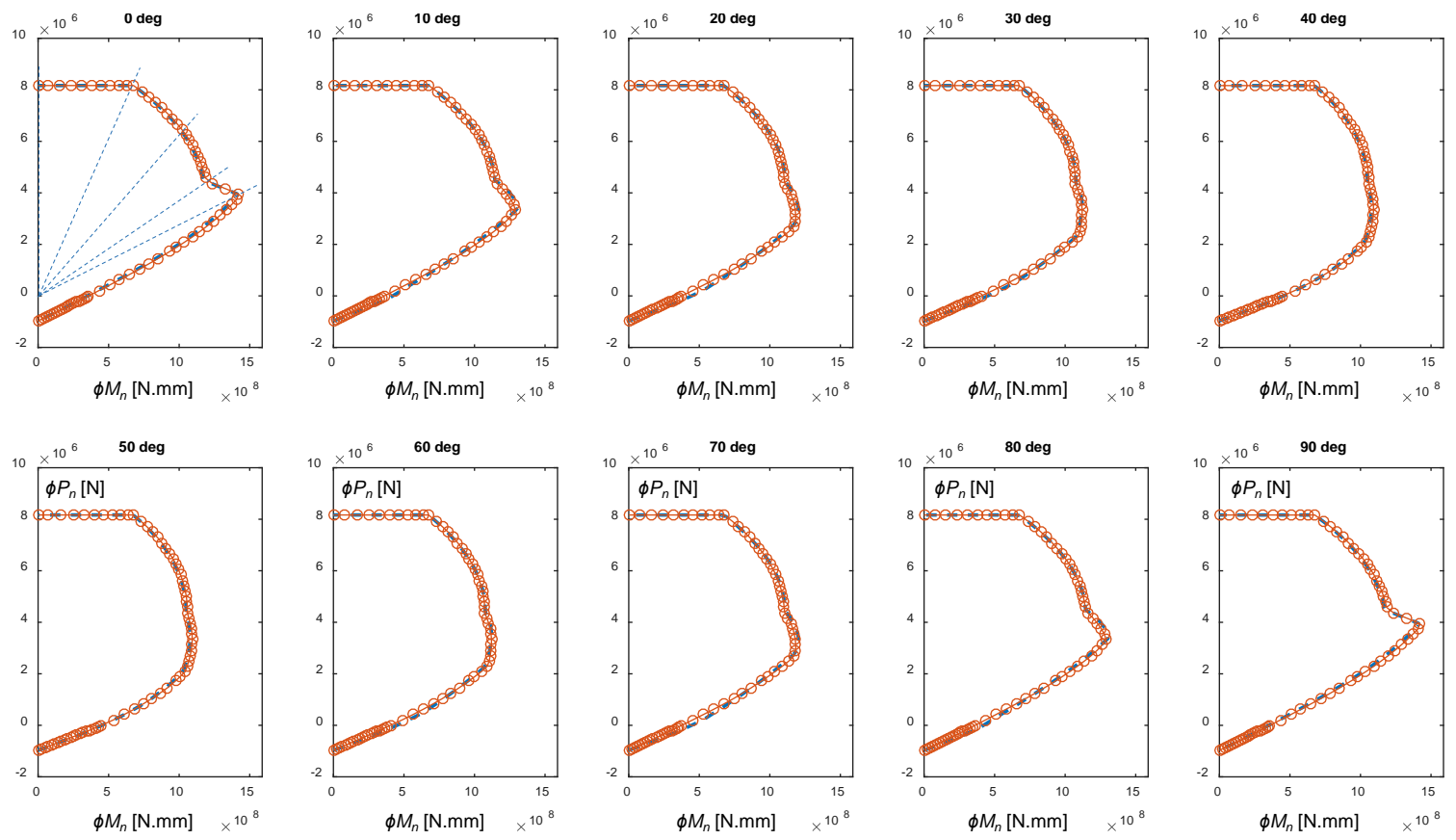

Figure A3.2. Comparisons of PM interaction diagrams as a function of angle $\theta$ 


\section{A3.1. Example section 2}

Figures A3.3 and A3.4 show the example section 2 of the $500 \mathrm{~mm} \times 800 \mathrm{~mm}$ rectangular column section with 16 reinforcements and the comparisons of PM interaction diagrams with respect to the angle $\theta$ from both the DAD_ESWL and the spColumn, respectively. The results obtained from the two programs with respect to the essential five points are compared based on the distance from origin to each point. The ratios of the DAD_ESWL to the spColumn for each point are then calculated and averaged regarding each angle. It is found that the differences between the two programs are within $2 \%$ in all cases.

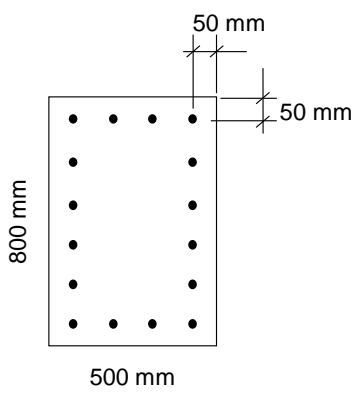

Figure A3.3. Example section 2 (Rectangular column)
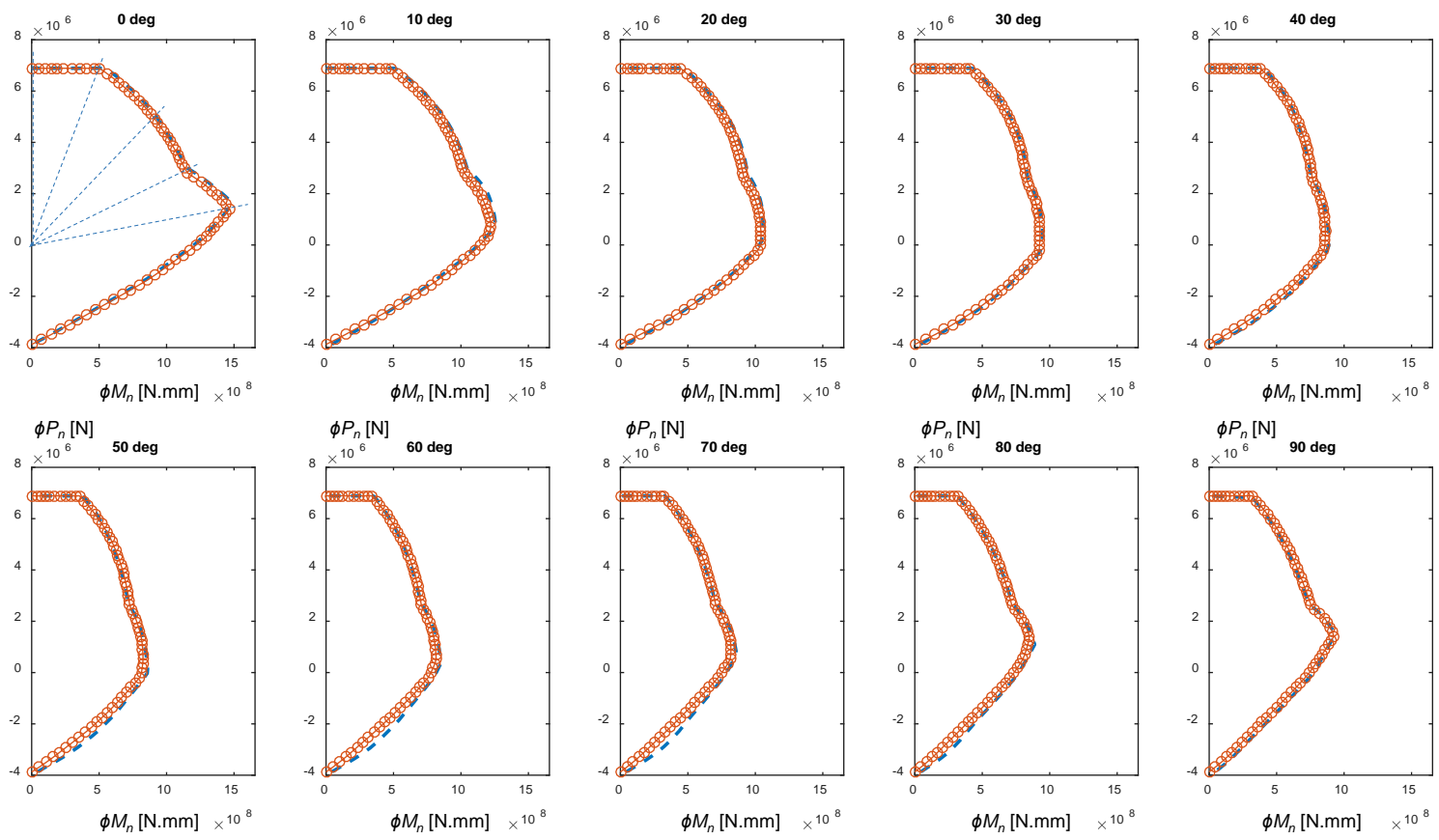

Figure A3.4. Comparisons of PM interaction diagrams as a function of angle $\theta$ 


\section{A3.1. Example section 3}

Figures A3.5 and A3.6 show the example section 3 of the $3810 \mathrm{~mm} \times 305 \mathrm{~mm}$ rectangular shear wall section with 24 reinforcements and the comparisons of PM interaction diagrams with respect to the angle $\theta$ from both the DAD_ESWL and the spColumn, respectively. The results obtained from the two programs with respect to the essential five points are compared based on the distance from origin to each point. The ratios of the DAD_ESWL to the spColumn for each point are then calculated and averaged regarding each angle. It is found that the differences between the two programs are up to $2 \%$ in all cases.

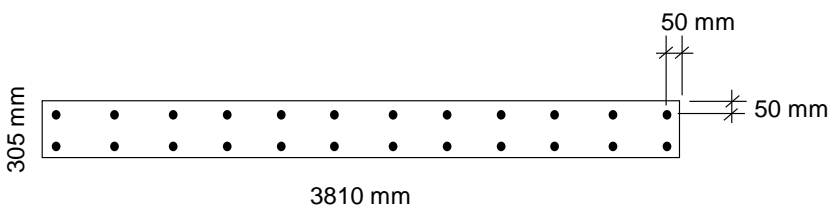

Figure A3.5. Example section 3 (Rectangular shear wall)
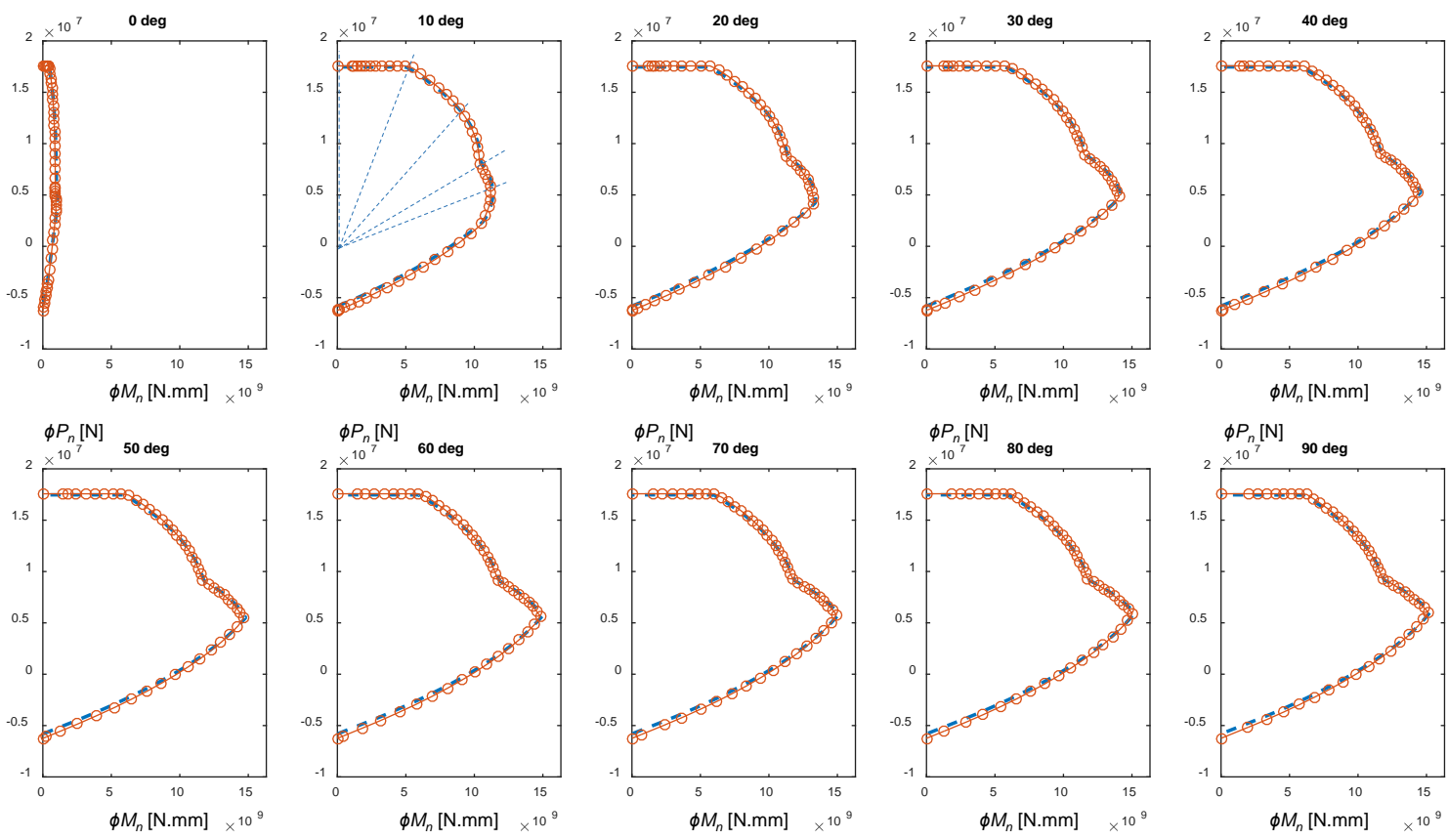

Figure A3.6. Comparisons of PM interaction diagrams as a function of angle $\theta$ 


\section{Appendix IV. Example Tcl script for running OpenSees}

The example Tcl script, 'ST_Frame3D_predefined.tcl' in the example, will be used to describe the inputs for executing OpenSees software in the DAD_ESWL platform. This appendix explains the minimum requirements for making the user's pre-defined Tcl script file, such as a basic structure, required commands, and properties. For more information, see the OpenSees Wiki ${ }^{3}$.

The Tcl script should start with the basic model builder (command: [model BasicBuilder]) for construction of the basic builder object. The [BasicBuilder] command includes the dimension of a problem ('-ndm' $=1,2$, or 3 ) and the number of degrees of freedom at nodes ('-ndf' $=6$ ) in the dimension assigned by the ndm option. The circular constant (pi, $\pi$ ) is directly defined. The command [puts] writes user-defined strings (e.g., "Set-up done ...", "Nodal coordinates defined ...", etc.) on a pop-up window from the console once the analysis progress by the OpenSees is done.

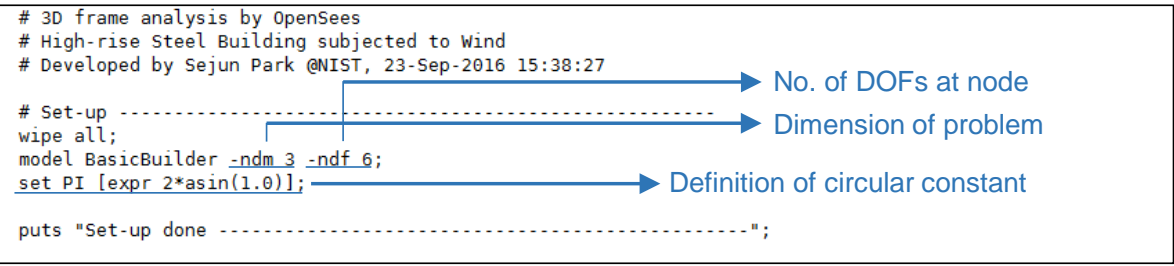

The command [node] is used to construct a Node object. It assigns nodal coordinates to the Node object. Total 3,260 nodes are used for steel high-rise building in this example. This command includes the integer tag identifying node (node label) and the nodal coordinates depending upon the 'ndm'.

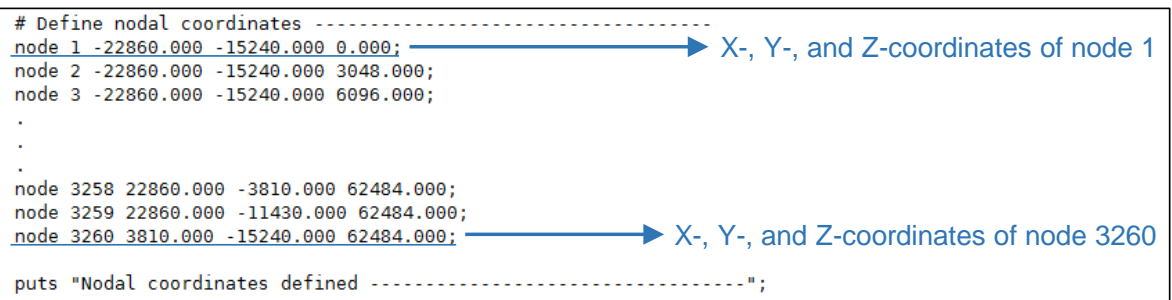

The command [fix] is used to construct homogeneous single-point boundary constraints. It is followed by 1) the master node located in the mass center of a rigid diaphragm on each floor and 2) constraints of 6 DOFs. The command includes the integer tag identifying the node to be constrained, and the constrained type represented by 1 (constrained) and 0 (unconstrained) with respect to each DOF. The command [rigidDiaphragm] is then used to construct a number of multi-point constraint objects. In the first line using 'rigidDiaphragm,' for example, ' 3 ' followed by the command represents the direction perpendicular to the rigid plane (i.e., 3 corresponds to the 1-2 plane) and sets the constraints so that the master node of ' 1039 ' and the slave nodes (' 1100 ', ' 1161 ', ...) move as in a rigid plane. For definition of the supports of the steel high-rise building in this

\footnotetext{
${ }^{3}$ http://opensees.berkeley.edu/wiki/index.php/Main_Page
} 
example, the command [fixZ] is used to construct multiple constraints for all nodes whose Z-coordinate lies within a specified distance from a specified coordinate. Namely, this command is used when specifying boundary conditions for a series of nodes lying in a plane parallel to the $\mathrm{X}-\mathrm{Y}$ plane. This example sets to fully constrain all the nodes in the X$\mathrm{Y}$ plane at $\mathrm{Z}=0.0$.

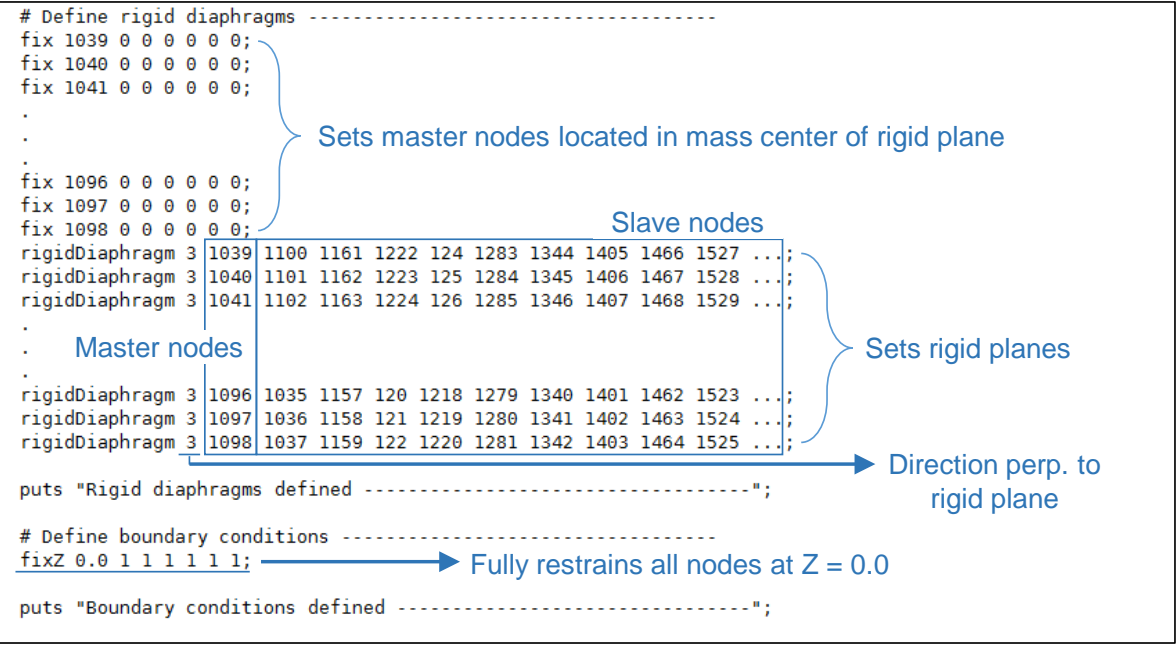

The next part defines sections. After defining tag numbers identifying the unique section object for each section by using the command [set], the command [section] is used to construct a Section Force Deformation object which describes force-deformation relationship at beam-column elements. By using this command, the elastic sectional and material properties are assigned to each section's tag number. The [section Elastic] command includes the section tag number, the Young's modulus ( $E,[\mathrm{MPa}])$, the cross sectional area of section $\left(\left[\mathrm{mm}^{2}\right]\right)$, the moment of inertia about the local $z$-axis $\left(I_{z},\left[\mathrm{~mm}^{4}\right]\right)$, the moment of inertia about the local $y$-axis $\left(I_{y},\left[\mathrm{~mm}^{4}\right]\right)$, the shear modulus ( $\left.G,[\mathrm{MPa}]\right)$, and the torsional moment of inertia of section $\left(\mathrm{J},\left[\mathrm{mm}^{4}\right]\right)$.

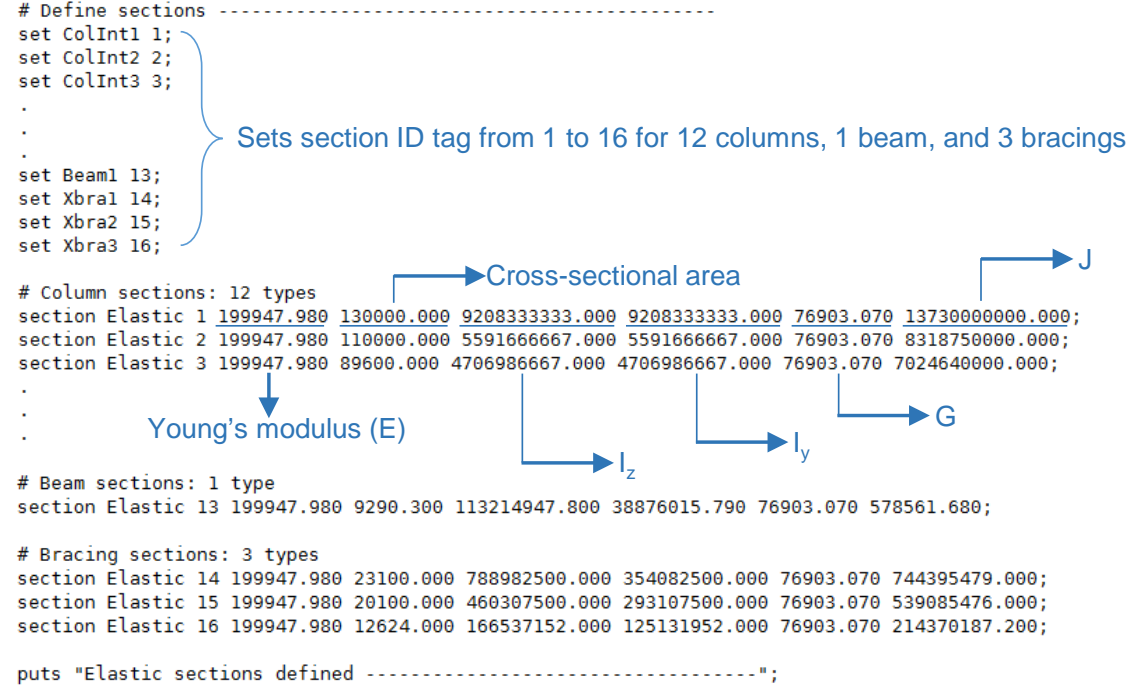


The command [geomTransf] is used to construct a coordinate-transformation object which transforms beam-column element stiffness and resisting force from the localcoordinate system to the global-coordinate system. This example applies a linear transformation for a three-dimensional problem. The command [geomTransf Linear] includes the unique identifier for Transf object and $\mathrm{X}, \mathrm{Y}$, and Z global-coordinate components of the 'vecxz' vector defined in the OpenSees manual (e.g., the local $z$ axis in this example). In this steel high-rise building example, total 7 geometric transformations are defined depending upon the directions and positions of the local $z$ axis for each member. For more information, refer to the OpenSees Wiki.

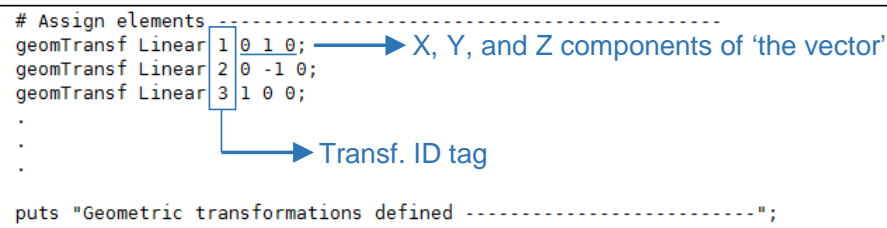

Finally, the beam-column elements should be defined for the building frames. The command [element nonlinearBeamColumn] is used. Total 8,140 elements are used for this steel high-rise building example. Note that the users must contain the command [-mass] which defines the element mass density (mass per unit length of a member, $[\mathrm{kg} / \mathrm{mm}]$ ). This element mass density will be used to form a global mass matrix of the system for solving the eigen problem. The command [element nonlinearBeamColumn] includes the unique element object identifier, the labels of end nodes, the number of integration points along the element, the identifier for the user-defined section object, the identifier for the userdefined geometric transformation object, and the [-mass] command with the element mass density.

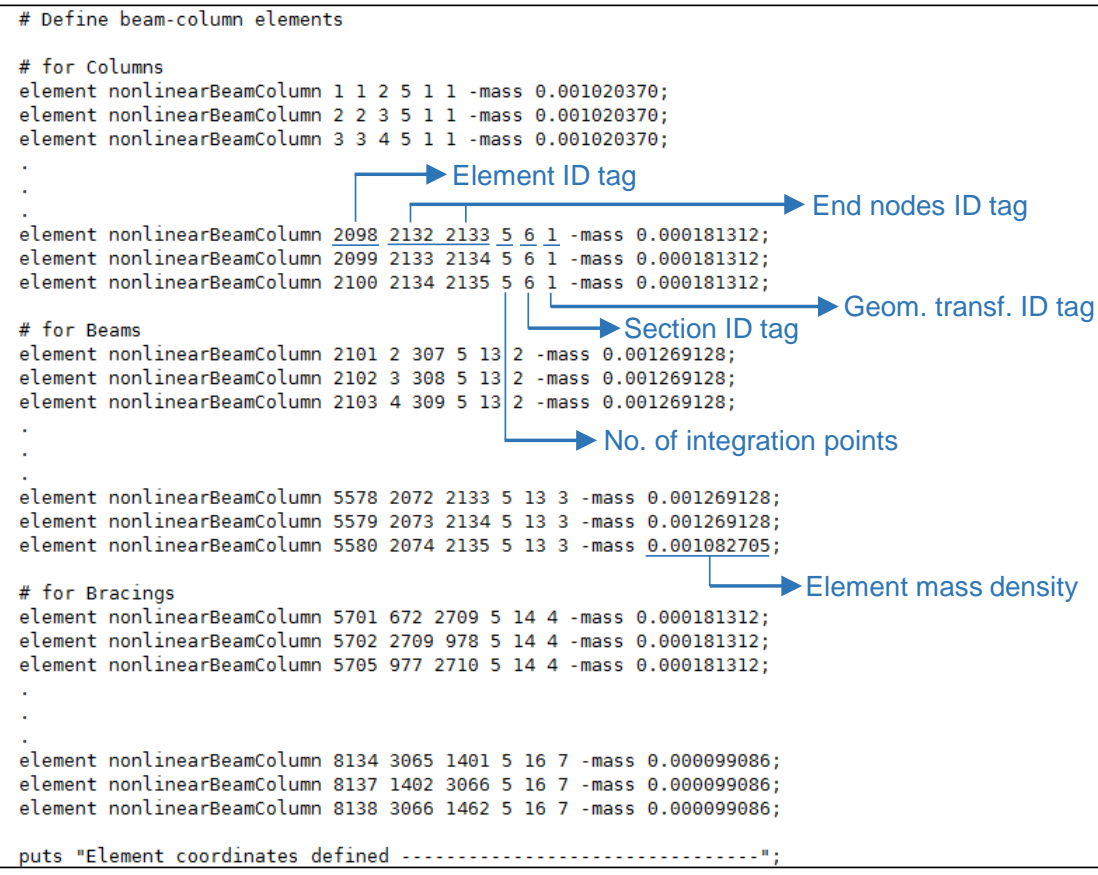


After inputting on the page BIdg. modeling the users' pre-defined Tcl script for their target high-rise building model, the click of button Run OpenSees generates the rest of mandatory objects, such as recorder, analysis, and time-series for running the OpenSees, and automatically adds them to the Tcl script. The final Tcl script is saved on the directory specified by the users with the filename of 'applyingLoads.tcl'. Then the button shows the string Running OpenSees ... busy, as shown below. This might take a few minutes to even hours according to the size of the problem and the computing system's performance. For reference, it took seven minutes to solve the steel high-rise building example (60-story with total 8,140 members) being stated in this manual by using a personal computer (Intel ${ }^{\circledR}$ Xeon ${ }^{\circledR}$ E5 CPU and 16 GB of RAM).

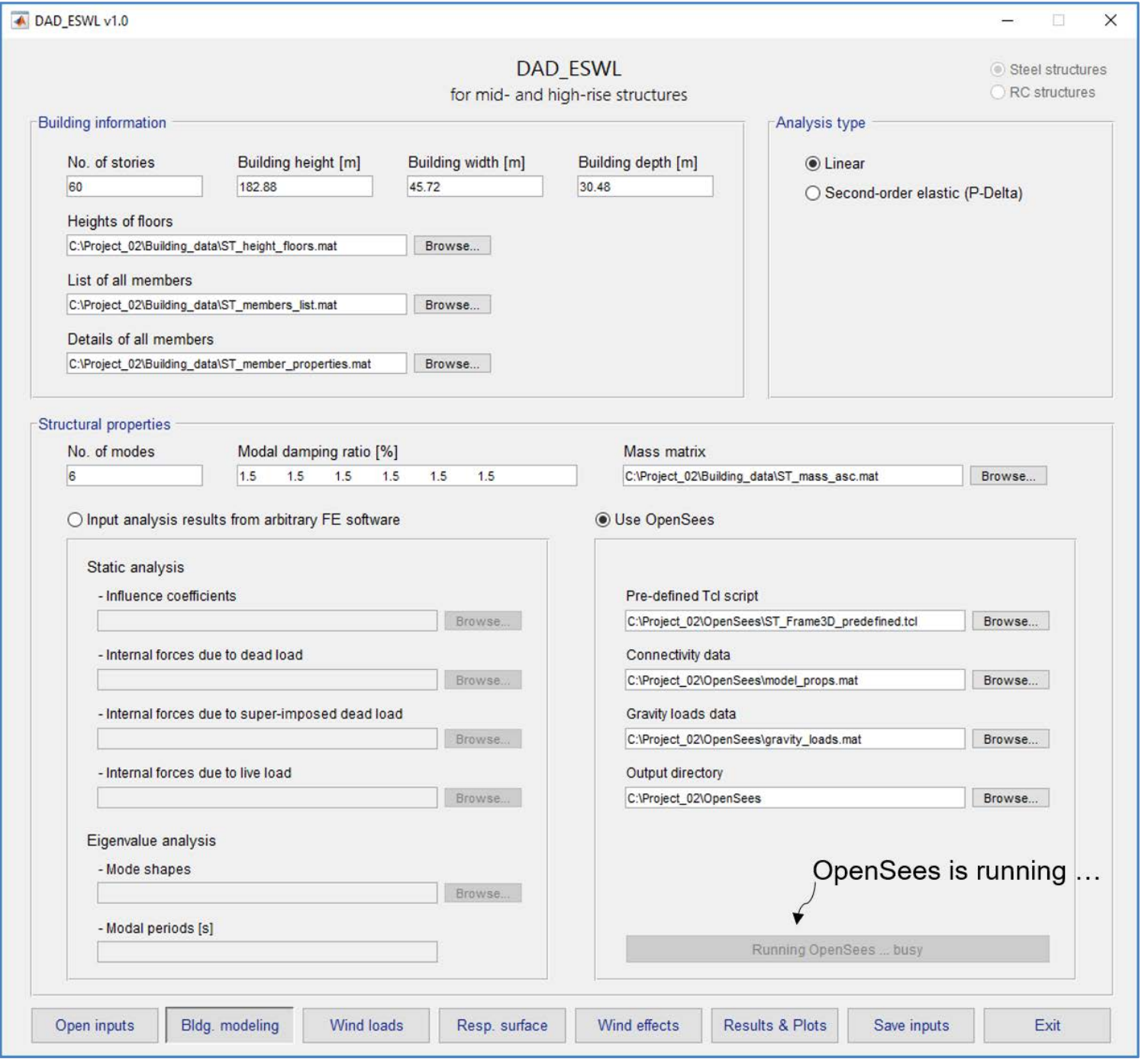

After running the analyses by the OpenSees, the DAD_ESWL displays a pop-up window including the analysis progress and results. If the users add to their Tcl script the command [puts] to check the analysis progress as described in the second paragraph in this appendix, those user-defined strings can be seen on this pop-up window. It also shows the 
natural periods of vibration, the applied load cases, and the elapsed time. These contents are saved on the directory specified by the users with the filename of 'Progress.txt'.

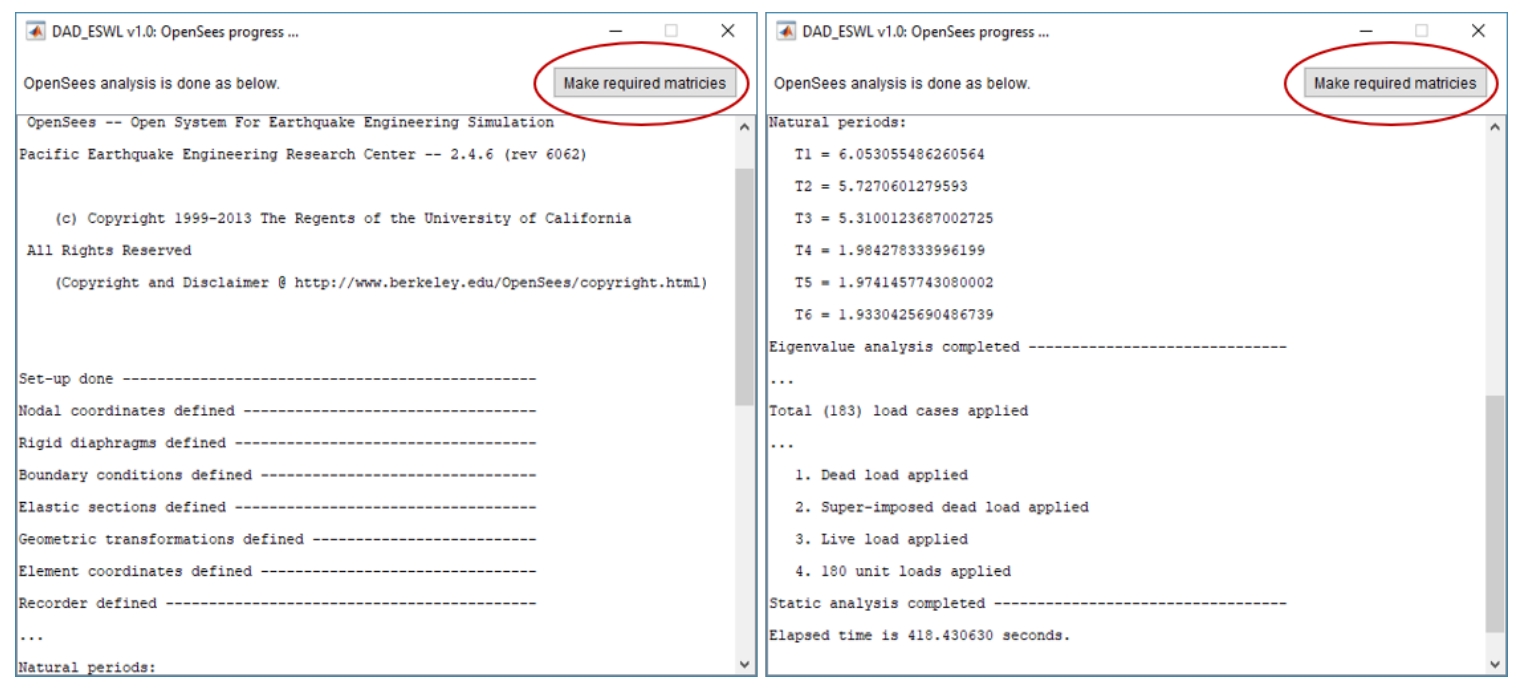

After checking all the information displayed on this window shown above, the users can generate the matrices of the results from the OpenSees analysis in appropriate forms of MATLAB data files to be used in the main algorithm of the DAD_ESWL by clicking the button Make required matrices located in the right upper of the window (red circles on figure above). Creating these matrices is a three-step process and the user can see a progress bar on each step: 1) Reading OpenSees output files, 2) Making matrices of frame internal forces due to gravity loads, and 3) Making a matrix of influence coefficients.

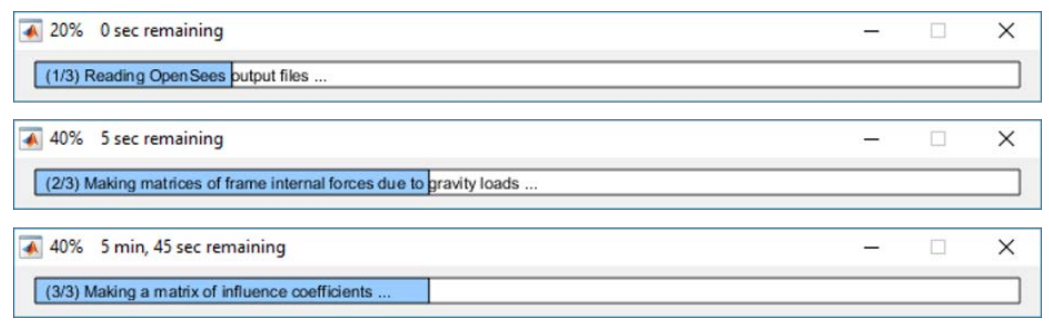

After all these calculations, the DAD_ESWL displays a dialog window as shown below. The button Close all close the pop-up window showing the progress and results and the dialog window. The users then can proceed to the next page Wind loads.

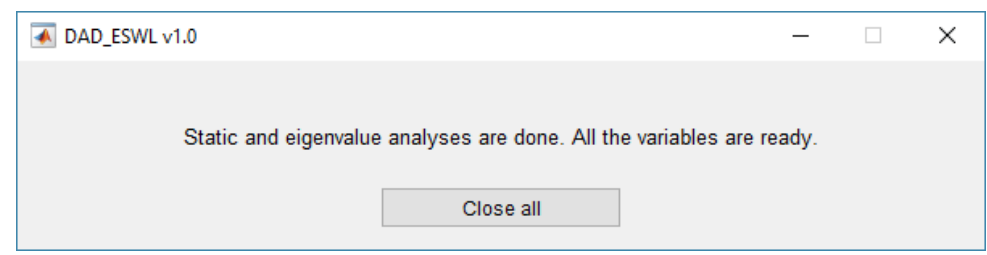




\section{Appendix V. Construction of variable - 'mass'}

This section briefly presents, through a simple example, a procedure for constructing the variable mass required to perform dynamic analyses by the DAD_ESWL software.

Figure A5.1 illustrates the 3-story steel building being considered, with $9 \mathrm{~m}$ height, 12 $\mathrm{m}$ width, and $12 \mathrm{~m}$ depth. The dynamic analyses performed by the DAD_ESWL software require the use of the lumped-mass system representation of the structure (Fig. A5.1c). The lumped masses are located at the building's center of mass, that is, at the intersection of the building's principal axes $x$ and $y$ and rotational $z$ axis. The following tasks show how the mass and the mass moment of inertia are calculated for each floor, and how the variable mass is constructed for use in the DAD_ESWL software.

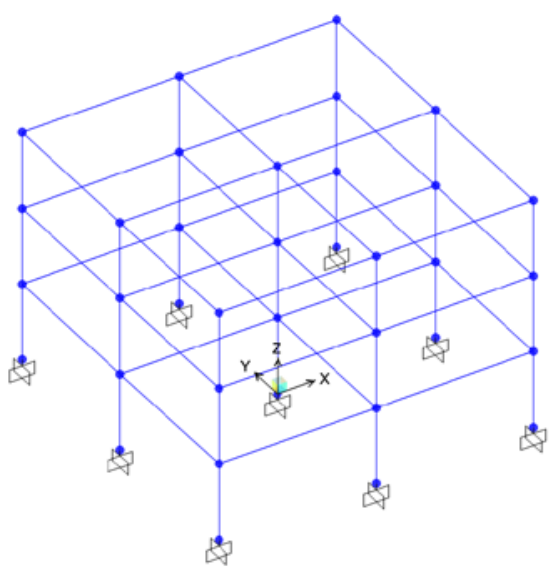

(a) 3D view

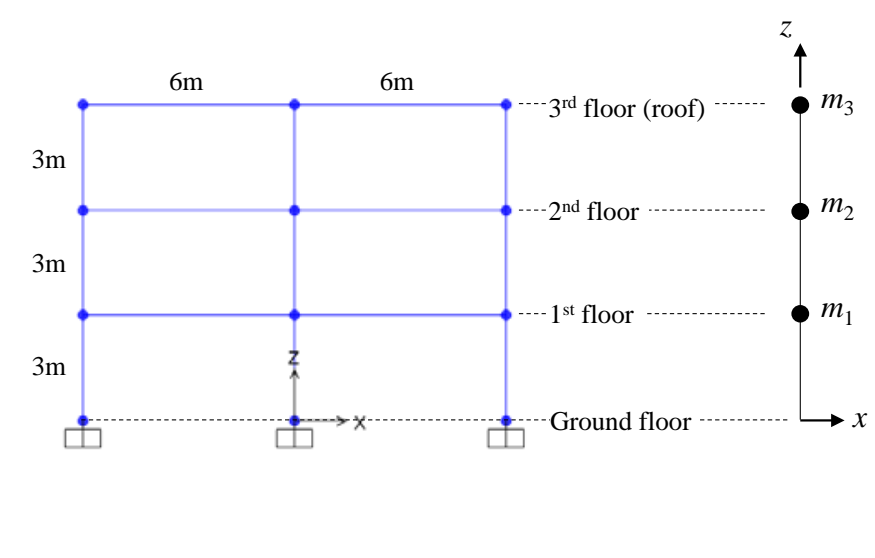

(b) Side view

(c) Lumped-mass system

Figure A5.1. Schematic views of 3-story example building 


\section{A5.1. Mass}

1. Determine the members' dimensions and other properties. The example 3-story steel structure consists of 9 columns per story (total 27 columns), 12 beams per floor (total 36 beams), and a concrete slab with $0.2 \mathrm{~m}$ thickness and $144 \mathrm{~m}^{2}$ area per floor. Assume that all columns and beams are W14x90 and W14x26, respectively, and that a super-imposed dead load of $80 \mathrm{~kg} / \mathrm{m}^{2}$ is applied to the slab. The specific masses of steel and reinforced concrete are $7849 \mathrm{~kg} / \mathrm{m}^{3}$ and $2406 \mathrm{~kg} / \mathrm{m}^{3}$, respectively.

2. Determine for each floor the tributary height in the lumped-mass model. It is assumed that the mass of each floor is concentrated at the elevation of the respective slab. Figure A5.2 shows the tributary height corresponding to the mass of each floor, $m_{1}, m_{2}$, and $m_{3}$. The mass corresponding to the ground floor (tributary height $=1.5 \mathrm{~m}$ from the ground) is not considered.

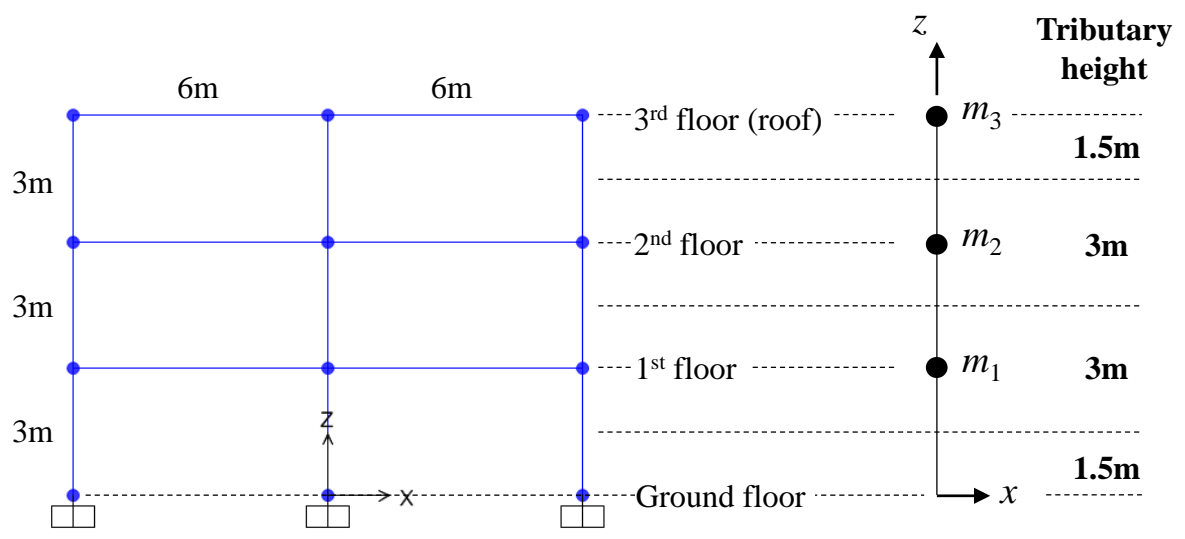

Figure A5.2. Tributary height for each floor of the lumped-mass system

3. Determine total mass per floor. Using the information given above, the mass per floor can be calculated as shown in the following table. The masses in $x$-and $y$-axis for the first, second, and roof floors are $85821 \mathrm{~kg}, 85821 \mathrm{~kg}$, and $83317 \mathrm{~kg}$, respectively.

\begin{tabular}{|c|c|c|c|c|c|c|c|c|c|c|}
\hline \multirow{3}{*}{ Floor } & \multirow{3}{*}{$\begin{array}{c}\text { Tributary } \\
\text { height }\end{array}$} & \multicolumn{3}{|c|}{ Column (W14x90) } & \multicolumn{3}{|c|}{ Beam (W14x26) } & \multirow{2}{*}{$\begin{array}{l}\text { Con'c } \\
\text { slab }\end{array}$} & \multirow{2}{*}{$\begin{array}{c}\text { Super- } \\
\text { imposed } \\
\text { dead } \\
\text { load }\end{array}$} & \multirow{2}{*}{$\begin{array}{l}\text { Total } \\
\text { mass } \\
\text { per } \\
\text { floor }\end{array}$} \\
\hline & & Area & $\begin{array}{c}\text { Mass } \\
\text { /member }\end{array}$ & \multirow{2}{*}{ EA } & Area & $\begin{array}{c}\text { Mass } \\
\text { /member }\end{array}$ & EA & & & \\
\hline & & {$\left[\mathrm{m}^{2}\right]$} & {$[\mathrm{kg}]$} & & {$\left[\mathrm{m}^{2}\right]$} & {$[\mathrm{kg}]$} & & {$[\mathrm{kg}]$} & {$[\mathrm{kg}]$} & [kg] \\
\hline Roof & 1.5 & 0.017 & 201.3 & 9 & 0.005 & 57.7 & 12 & 69292.8 & 11520.0 & 83317.0 \\
\hline 2 & 3.0 & 0.017 & 402.7 & 9 & 0.005 & 115.4 & 12 & 69292.8 & 11520.0 & 85821.2 \\
\hline 1 & 3.0 & 0.017 & 402.7 & 9 & 0.005 & 115.4 & 12 & 69292.8 & 11520.0 & 85821.2 \\
\hline Ground & 1.5 & 0.017 & 201.3 & 9 & 0.000 & 0.0 & 0 & 0 & 0 & 1811.9 \\
\hline
\end{tabular}




\section{A5.2. Mass moment of inertia}

Each member is assumed to be a thin rod. Therefore, the mass moment of inertia for column members is $I_{\text {col }}=m_{c} r^{2}$, where $m_{c}$ is the mass of the column and $r$ is the distance from the rotation axis (z-axis of the building) to the member's mass center. For the beam members, the case of a thin rod about its center should be included, thus, $I_{\text {beam }}=m_{b} r^{2}+m_{b} I^{2} / 12$, where $m_{b}$ is the mass of the beam and $l$ is the beam length.

1. Classify the members by their mass moment of inertia. Using the symmetry of the building section, the members can be classified as shown in Fig. A5.3 ( $c_{1}$ and $c_{2}$ for columns and $b_{1}, b_{2}, b_{3}$, and $b_{4}$ for beams). Note that the length of the beams is $l=12 \mathrm{~m}$.

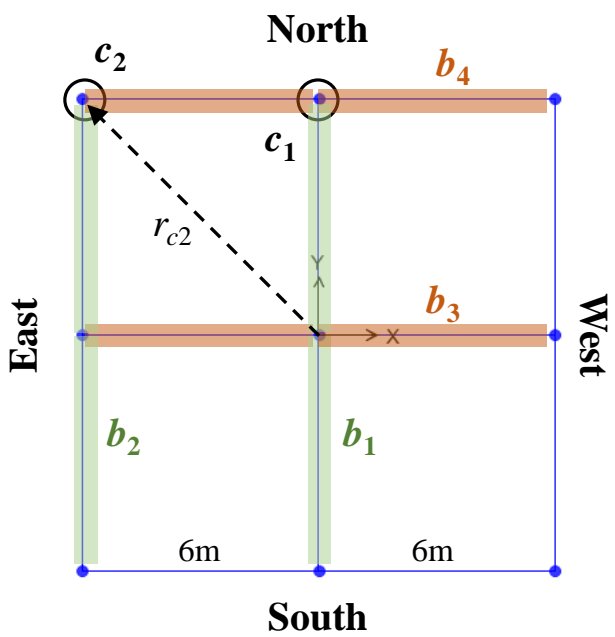

Figure A5.3. Categorized members for the mass moment of inertia in the plan view.

The mass moment of inertia for each type of member is calculated as follows:

$$
\begin{aligned}
& I_{c o l}=4 \sum_{i=1}^{2} m_{c i} r_{c i}^{2} \quad \text { for columns } \\
& I_{b e a m, N S}=2\left(m_{b 2} r_{b 2}^{2}+m_{b 2} \frac{l^{2}}{12}\right)+m_{b 1} \frac{l^{2}}{12} \quad \text { for beams located vertically, i.e., } b_{1} \text { and } b_{2} \\
& I_{\text {beam }, E W}=2\left(m_{b 4} r_{b 4}^{2}+m_{b 4} \frac{l^{2}}{12}\right)+m_{b 3} \frac{l^{2}}{12} \quad \text { for beams located horizontally, i.e., } b_{3} \text { and } b_{4}
\end{aligned}
$$


2. Determine the mass moment of inertia for columns and beams. Using the information given above, the mass moment of inertia per floor can be calculated as shown in the following table.

\begin{tabular}{|c|c|c|c|c|c|}
\hline \multirow{2}{*}{ Floor } & Tributary height & Column & Beam (NS) & Beam $(\mathrm{EW})$ & Total \\
\cline { 2 - 6 } & {$[\mathrm{m}]$} & {$\left[\mathrm{kg} \cdot \mathrm{m}^{2}\right]$} & {$\left[\mathrm{kg} \cdot \mathrm{m}^{2}\right]$} & {$\left[\mathrm{kg} \cdot \mathrm{m}^{2}\right]$} & {$\left[\mathbf{k g} \cdot \mathbf{m}^{2}\right]$} \\
\hline Roof & 1.5 & 115964.3 & 6230.5 & 6230.5 & $\mathbf{1 2 8 4 2 5 . 3}$ \\
\hline 2 & 3.0 & 231928.5 & 12461.1 & 12461.1 & $\mathbf{2 5 6 8 5 0 . 7}$ \\
\hline 1 & 3.0 & 231928.5 & 12461.1 & 12461.1 & $\mathbf{2 5 6 8 5 0 . 7}$ \\
\hline Ground & 1.5 & 115964.3 & 0.0 & 0.0 & 115964.3 \\
\hline
\end{tabular}

3. Determine the mass moment of inertia for the concrete slab and super-imposed dead loads. Let the concrete slab and the super-imposed dead loads assume to be flat planes. The mass moments of inertia for them can be calculated as follows:

$$
\begin{array}{ll}
I_{\text {slab }}=\frac{1}{12} m_{s}\left(a^{2}+b^{2}\right) & \text { for concrete slab } \\
I_{\text {sdead }}=\frac{1}{12} m_{\text {sd }}\left(a^{2}+b^{2}\right) & \text { for super-imposed dead load }
\end{array}
$$

where $m_{s}$ is the mass of the concrete slab, $m_{s d}$ is the mass of the super-imposed dead load, and $a$ and $b$ are the width and depth of the concrete slab ( $a=b=12 \mathrm{~m}$ in this example). Using the information given above, the mass moment of inertia per floor can be calculated as shown in the following table.

\begin{tabular}{|c|c|c|c|c|}
\hline \multirow{2}{*}{ Floor } & Tributary height & Con'c slab & Super-imposed dead load & Total \\
\cline { 2 - 5 } & {$[\mathrm{m}]$} & {$\left[\mathrm{kg} \cdot \mathrm{m}^{2}\right]$} & {$\left[\mathrm{kg} \cdot \mathrm{m}^{2}\right]$} & {$\left[\mathbf{k g} \cdot \mathbf{m}^{2}\right]$} \\
\hline Roof & 1.5 & 1663027.2 & 276480.0 & $\mathbf{1 9 3 9 5 0 7 . 2}$ \\
\hline 2 & 3.0 & 1663027.2 & 276480.0 & $\mathbf{1 9 3 9 5 0 7 . 2}$ \\
\hline 1 & 3.0 & 1663027.2 & 276480.0 & $\mathbf{1 9 3 9 5 0 7 . 2}$ \\
\hline Ground & 1.5 & 0.0 & 0.0 & 0.0 \\
\hline
\end{tabular}

4. Determine total mass moment of inertia per floor. The total mass moments of inertia in the rotational $z$ axis are a sum of those for the structural members and for the concrete slab and the super-imposed dead load. Therefore, those for the first, second, and roof floors are $2196358 \mathrm{~kg} \cdot \mathrm{m}^{2}, 2196358 \mathrm{~kg} \cdot \mathbf{m}^{2}$, and $20679323 \mathrm{~kg} \cdot \mathbf{m}^{2}$, respectively. 


\section{A5.3. MATLAB variable mass}

The variable mass is a column vector whose components consist of the masses and mass moment of inertia associated with each floor of the building. The first 3 components are associated with the masses $(\mathrm{kg})$ contained in the building's principal axes $x$ and $y$, and with the mass moment of inertia $\left(\mathrm{kg} \cdot \mathrm{m}^{2}\right)$ about the building's rotational $z$-axis at the first floor. The next 3 components are associated with the second floor and so forth (see Fig. A5.4). Therefore, if the number of floors is $N$, the mass vector will have $3 N$ components.
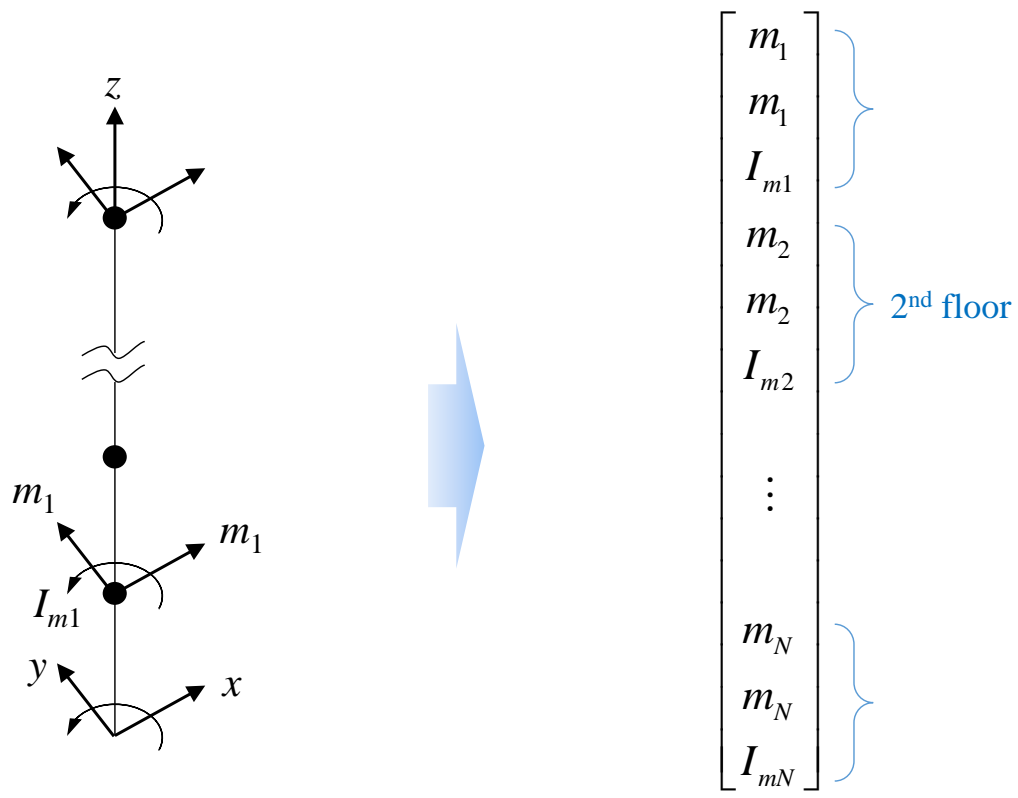

Figure A5.4. Variable - mass

Therefore, the variable mass for the example building is

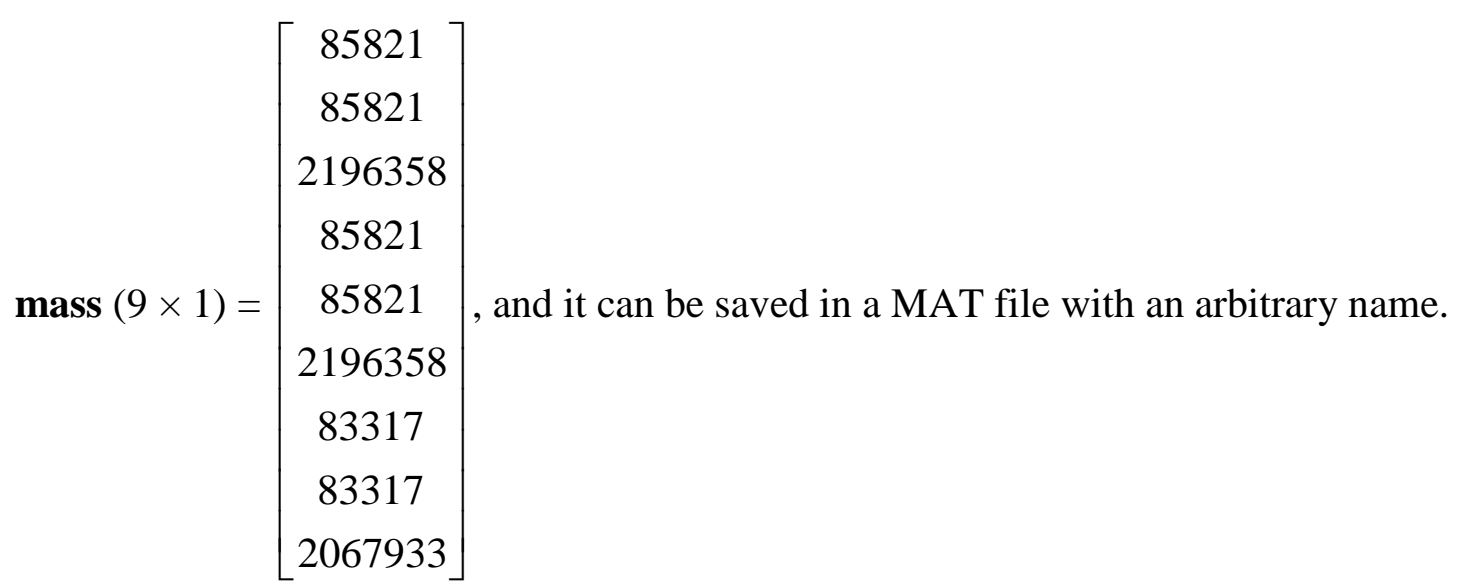




\section{Appendix VI. Construction of variable - 'dif'}

This section briefly presents a procedure for constructing the variable dif required to perform dynamic analyses by the DAD_ESWL software, where dif denotes influence coefficients representing internal forces in the structure's members induced by a unit load with 3 components (along the $x, y$, and $z$ axes) acting at the mass center of each floor. The example building used to illustrate the procedure is the same as in Appendix V (Fig. A.5.1).

The internal forces in the members are the axial force (P), shear forces in the member's local $x$ and $y$ directions $\left(\mathrm{V}_{2}\right.$ and $\left.\mathrm{V}_{3}\right)$, torsional moment $(\mathrm{T})$, and bending moments about the member's local $x$ and $y$ directions $\left(\mathrm{M}_{2}\right.$ and $\left.\mathrm{M}_{3}\right)$. The influence coefficients will be used for determining the members' wind-induced internal forces via multiplication by multiplying them to the effective floor wind loads.

The following tasks show how the influence coefficients are calculated and how the variable dif is constructed.

Note that the influence coefficients of a building model can be obtained using a finite element analysis software. In this report, SAP 2000 (CSI 2015) was used for the calculation of the influence coefficients for all members.

1. Determine the members' dimensions and other properties. This task is identical to its counterpart in Appendix V.

2. Apply unit loads to the mass center of each floor. The unit loads with 3 directions, i.e., the unit forces $(1 \mathrm{~N})$ about the global $x$ and $y$ axes and the unit moment $(1 \mathrm{~N} \cdot \mathrm{m})$ about the global $z$-axis, should be applied to the mass center of each floor. For this example, a total of 9 load cases (3 directions time 3 floors) would be generated and applied to the building model as shown in Fig. A6.1.

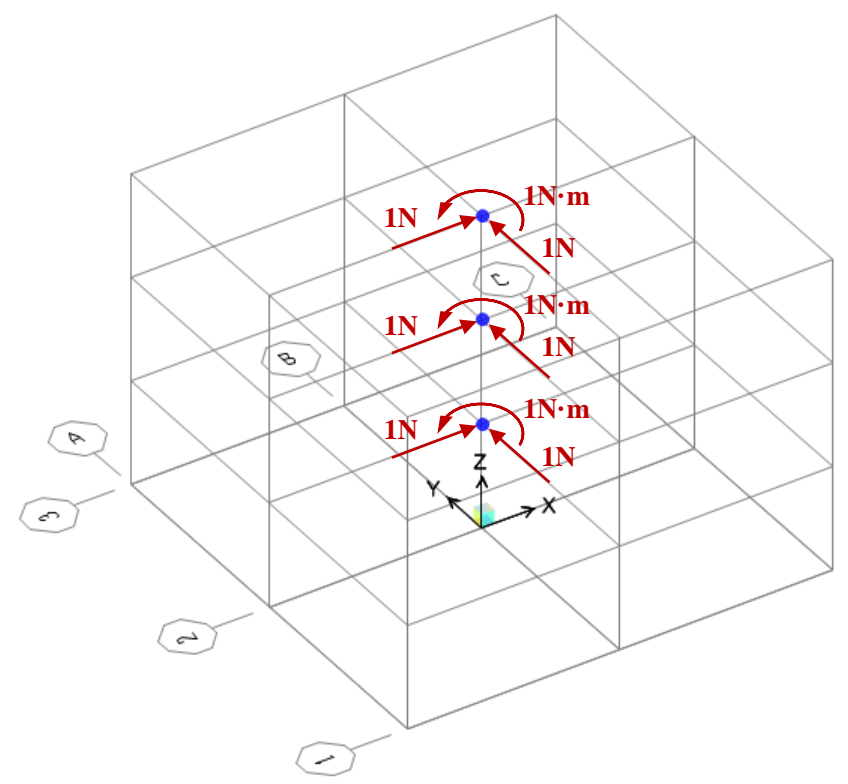

Figure A6.1. Applying unit loads to mass center of each floor

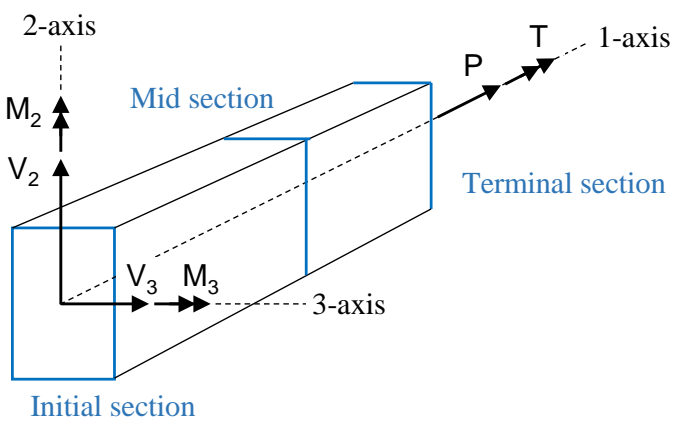

Figure A6.2. Element output stations and local axes 
3. Set the element output station to 3. The DAD_ESWL software is developed to obtain the internal forces at three sections of each structural member. The three locations are the member's two ends and the midpoint (shown as initial, terminal, and mid sections, respectively, in Fig. A6.3). The influence coefficients required to obtain the internal forces must be calculated accordingly.

4. Determine the influence coefficients. The 6 internal forces, i.e., $\mathrm{P}, \mathrm{V}_{2}, \mathrm{~V}_{3}, \mathrm{~T}, \mathrm{M}_{2}, \mathrm{M}_{3}$, at the 3 locations of each member can be obtained by a finite element analysis software program (SAP 2000 for this example), and can be shown as Fig. A6.3. The first column indicates the element labels from 1 to 63 (27 columns plus 36 beams), the second column indicates the output location of each member, and the third column indicates the unit load cases (unit_x01 to 03, unit_y01 to 03, and unit_z01 to 03 correspond to the unit forces in the global $x$ and $y$ axes and the unit moment about global $z$ axis, respectively). The next 6 columns indicate each of the 6 internal forces in sequence. A total number of the data rows should be 1701 (3 locations $\times 3$ unit loads $\times 3$ floors $\times 63$ members).

\begin{tabular}{|c|c|c|c|c|c|c|c|c|}
\hline Element & Location & Load case & $\mathbf{P}$ & V2 & V3 & $\mathbf{T}$ & M2 & M3 \\
\hline Label & $\mathrm{m}$ & Text & $\mathrm{N}$ & $\mathrm{N}$ & $\mathrm{N}$ & $\mathrm{N}-\mathrm{m}$ & $\mathrm{N}-\mathrm{m}$ & $\mathrm{N}-\mathrm{m}$ \\
\hline 1 & 0 & unit_x01 & 0.0029 & 0.0028 & 0.0001 & -0.0001 & 0.0001 & 0.0085 \\
\hline 1 & 1.5 & unit_x01 & 0.0029 & 0.0028 & 0.0001 & -0.0001 & 0.0000 & 0.0043 \\
\hline 1 & 3 & unit_x01 & 0.0029 & 0.0028 & 0.0001 & -0.0001 & -0.0001 & 0.0002 \\
\hline 1 & 0 & unit_x02 & 0.0084 & 0.0069 & 0.0001 & -0.0002 & 0.0002 & 0.0220 \\
\hline 1 & 1.5 & unit_x02 & 0.0084 & 0.0069 & 0.0001 & -0.0002 & 0.0000 & 0.0116 \\
\hline 1 & 3 & unit_x02 & 0.0084 & 0.0069 & 0.0001 & -0.0002 & -0.0002 & 0.0012 \\
\hline 1 & 0 & unit_x03 & 0.0136 & 0.0102 & 0.0002 & -0.0002 & 0.0003 & 0.0331 \\
\hline 1 & 1.5 & unit_x03 & 0.0136 & 0.0102 & 0.0002 & -0.0002 & -0.0001 & 0.0177 \\
\hline 1 & 3 & unit_x03 & 0.0136 & 0.0102 & 0.0002 & -0.0002 & -0.0004 & 0.0024 \\
\hline 1 & 0 & unit_y01 & 0.0053 & 0.0001 & 0.0044 & 0.0002 & 0.0103 & 0.0002 \\
\hline 1 & 1.5 & unit_y01 & 0.0053 & 0.0001 & 0.0044 & 0.0002 & 0.0037 & 0.0000 \\
\hline 1 & 3 & unit_y01 & 0.0053 & 0.0001 & 0.0044 & 0.0002 & -0.0029 & -0.0001 \\
\hline 1 & 0 & unit_y02 & 0.0138 & 0.0002 & 0.0100 & 0.0003 & 0.0240 & 0.0003 \\
\hline 1 & 1.5 & unit_y02 & 0.0138 & ก กกกר & nn1nn & $n m m$ & n nnnn & n nกnก \\
\hline & & & & & c.uvue & -u.vuUL & 0.0013 & 0.0026 \\
\hline 63 & 3 & unit_z01 & -0.0002 & 0.0004 & 0.0002 & -0.0002 & 0.0010 & 0.0020 \\
\hline 63 & 6 & unit_z01 & -0.0002 & 0.0004 & 0.0002 & -0.0002 & 0.0006 & 0.0014 \\
\hline 63 & 0 & unit_z02 & -0.0007 & 0.0006 & 0.0006 & -0.0017 & 0.0061 & 0.0083 \\
\hline 63 & 3 & unit_z02 & -0.0007 & 0.0006 & 0.0006 & -0.0017 & 0.0052 & 0.0074 \\
\hline 63 & 6 & unit_z02 & -0.0007 & 0.0006 & 0.0006 & -0.0017 & 0.0043 & 0.0065 \\
\hline 63 & 0 & unit_z03 & -0.0002 & -0.0103 & -0.0104 & 0.0020 & -0.0118 & -0.0114 \\
\hline 63 & 3 & unit_z03 & -0.0002 & -0.0103 & -0.0104 & 0.0020 & 0.0037 & 0.0040 \\
\hline 63 & 6 & unit_z03 & -0.0002 & -0.0103 & -0.0104 & 0.0020 & 0.0193 & 0.0195 \\
\hline
\end{tabular}

Figure A6.3. Table of influence coefficients output data

The output data can be obtained in various formats, e.g., Microsoft Excel (.xlsx), text (.txt), or comma separated values (.csv). 
5. Make the MATLAB variable - dif. The output data saved in an arbitrary format mentioned above can be read by using the MATLAB functions, e.g., 'fopen', 'fgets', and 'xlsread'. Next, the data should be converted to an appropriate 3D array variable named dif for processing by the DAD_ESWL software. It is strongly recommended that the users write a script to handle the tasks of reading and converting the output data.

The variable dif is a 3D array. Each face of dif contains the influence coefficients associated with the six internal forces that are induced by a unit force or moment applied to the mass center of a given floor in one of the directions $x, y$, or rotational $z$. The internal forces are assigned from the first column to the sixth column, such as $P, V_{2}, V_{3}, T, M_{2}$, and $M_{3}$. The first 3 (directions) $\times 3$ (floors) $=9$ rows contain influence coefficients in the initial section. The second and the third 9 rows contain the influence coefficients in the midsection and in the terminal-section, respectively. Each 3-row contains influence coefficients in the $x$ axis first from the first floor to the roof floor, then those in the $y$ direction, and finally those in the rotational $z$ direction. The total $9 \times 3$ (output locations per member) $=27$ rows are assigned in every column for a given face. The index of each face identifies the member.

Finally, the array size for this example is 27 rows $\times 6$ columns $\times 63$ faces. Figure A6.4 shows the variable dif for the member 1 (out of 63) of the 3-story example building.

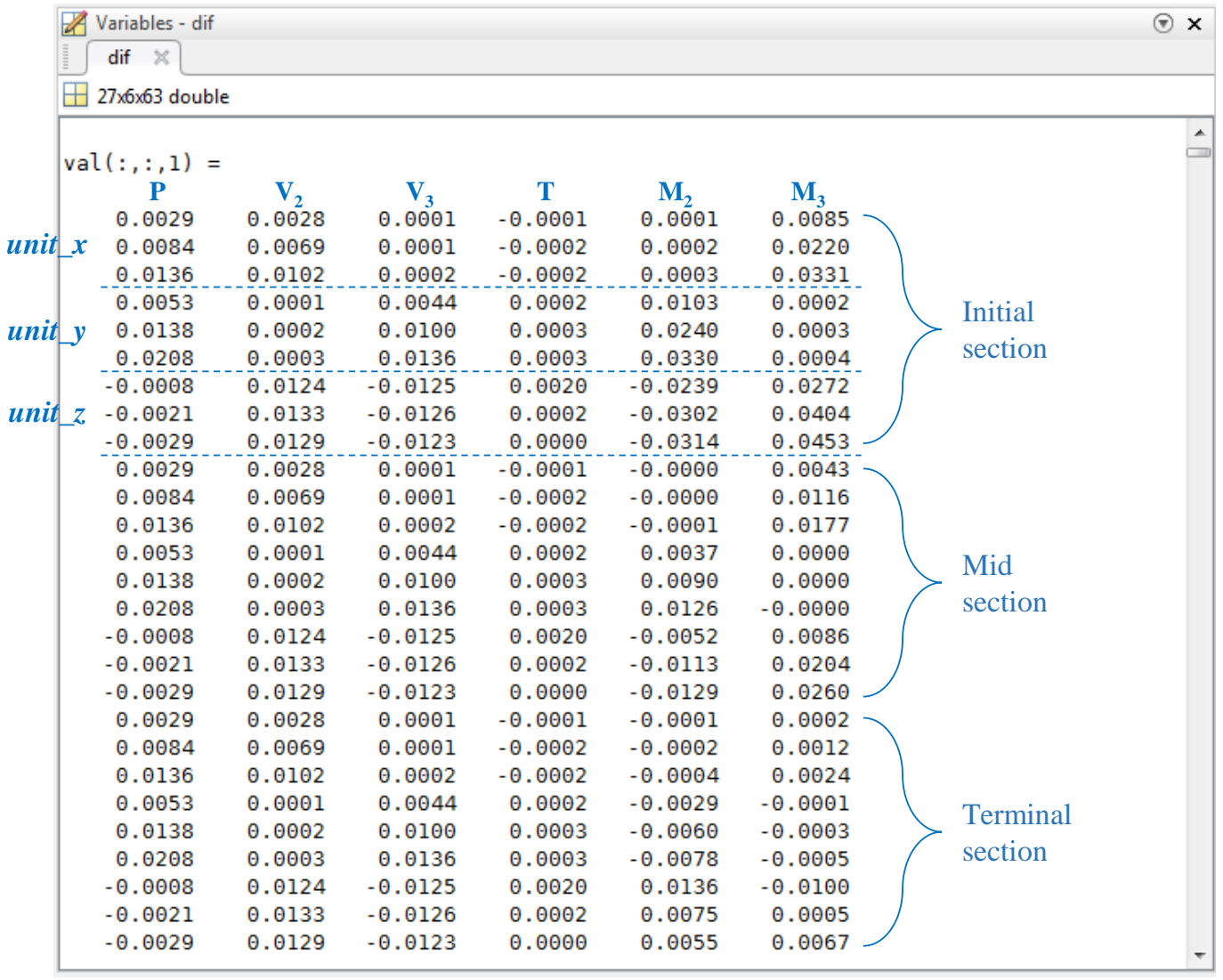

Figure A6.4. Variable - dif $(27 \times 6 \times 63)$ 


\section{Appendix VII. Construction of variable - 'Ratio_Vs'}

This section presents a procedure for constructing the variable Ratio_Vs required to perform the extreme wind speed analyses in the DAD_ESWL software. The extreme wind speed analyses must use micro-meteorologically homogeneous data, meaning that all the wind speed data in a set correspond to (1) the same height above ground, (2) with the same terrain exposure (e.g., open or suburban), and (3) the same averaging time (e.g., 3-s, 1-min, 10 -min, or 1-hour). If the wind speed data do not satisfy the micrometeorological homogeneity requirements, they should be transformed so that the requirements are satisfied. To transform them to the mean hourly wind speeds at the building height in the requisite terrain exposure, the wind speed ratios, i.e., the variable Ratio_Vs, for each terrain exposure should be used.

The MATLAB variable Ratio_Vs is a row vector containing the ratios between wind speeds in $\mathrm{m} / \mathrm{s}$ at the weather station (e.g., at $10 \mathrm{~m}$ above ground in open terrain exposure) to the mean hourly wind speeds at the building height for the terrain exposures of the building being designed. The variable must consist of four columns with respect to the terrain exposures as shown in Fig. A7.1. The following sections explain a relation between the wind speeds averaged over different time intervals above any type of terrain exposure and show examples of the calculation of those ratios.

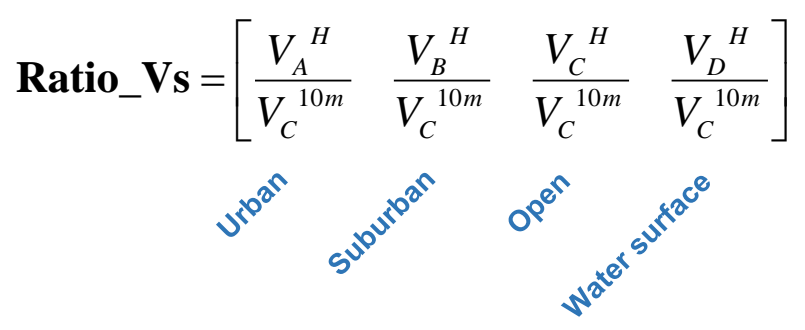

Figure A7.1. Variable structure

\section{A7.1. Wind speed profiles and micro-meteorological homogeneity}

The variation of wind speed with height can be expressed approximately by the power law as

$$
V(z)=V\left(z_{r e f}\right)\left(\frac{Z}{z_{r e f}}\right)^{\frac{1}{\alpha}}
$$

where $V$ is the wind speed, $z$ is the height, and $z_{r e f}$ is the reference height. The exponent $1 / \alpha$ depends upon surface roughness and the averaging time, the profiles being flatter as the averaging time decreases. The power law exponents are specified by the ASCE 7-16 standards (ASCE 2016) and by the National Building Code of Canada (Canada 1971), and will be mentioned at the end of this section. Figure A7.2 shows a schematic of the wind profiles for the open and arbitrary terrain exposures. 


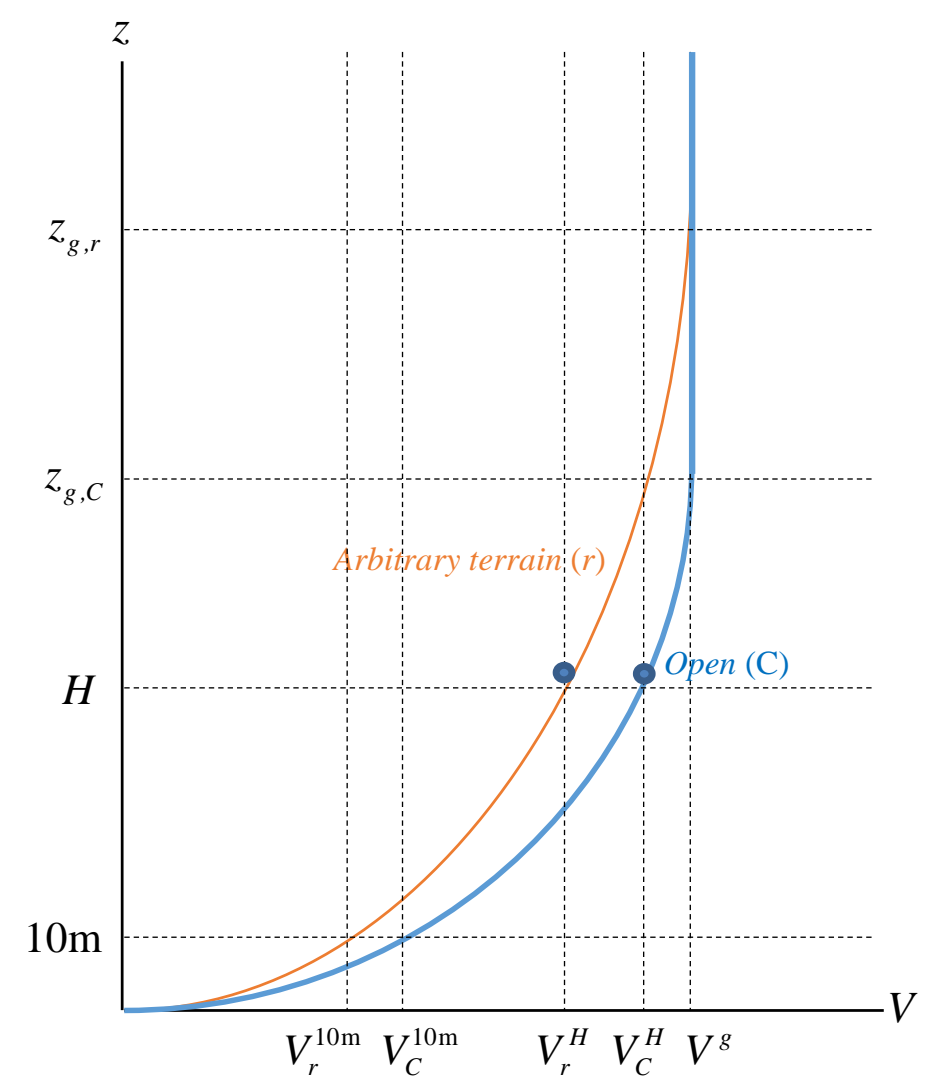

Figure A7.2. Schematic of wind profiles for the open and arbitrary terrain exposures.

From Eq. A7.1, the wind speed at the building height $(H)$ in the arbitrary terrain exposure ( $r=\mathrm{A}, \mathrm{B}, \mathrm{C}$, or $\mathrm{D})$ can be expresses as

$$
V_{r}^{H}=V_{r}^{10 \mathrm{~m}}\left(\frac{H}{10}\right)^{\frac{1}{\alpha_{r}}}
$$

where $V_{r}^{10 \mathrm{~m}}$ is the wind speed at the reference height (10 $\mathrm{m}$ above ground) in the arbitrary terrain exposure $\tau$, and it also can be expressed from Eq. A7.1 as

$$
V_{r}^{10 \mathrm{~m}}=V_{r}^{g}\left(\frac{10}{\mathrm{Z}_{g, r}}\right)^{\frac{1}{\alpha_{r}}}
$$

where $V_{r}^{g}$ is the wind speed at the gradient height in the arbitrary terrain exposure $\left(z_{g, r}\right)$. Since the gradient wind speed is independent of any type of terrain exposure (see Fig. A7.2), the following expression can be satisfied.

$$
V_{r}^{g}=V_{C}^{g}=V_{C}^{10 \mathrm{~m}}\left(\frac{Z_{g, C}}{10}\right)^{\frac{1}{\alpha_{C}}}
$$


where $V_{C}^{10 \mathrm{~m}}$ is the wind speed at the reference height in the open terrain exposure, which is the data obtained in the weather station, and $Z_{g, C}$ is the gradient height in the open terrain exposure (Table A7.1). Substituting Eq. A7.4 in Eq. A7.3 yields

$$
V_{r}^{10 \mathrm{~m}}=V_{C}^{10 \mathrm{~m}}\left(\frac{Z_{g, C}}{10}\right)^{\frac{1}{\alpha_{C}}}\left(\frac{10}{Z_{g, r}}\right)^{\frac{1}{\alpha_{r}}}
$$

Thus, Eq. A7.2 can be rewritten by substituting Eq. A7.5 in it as

$$
V_{r}^{H}=V_{C}^{10 \mathrm{~m}}\left(\frac{Z_{g, C}}{10}\right)^{\frac{1}{\alpha_{C}}}\left(\frac{H}{Z_{g, r}}\right)^{\frac{1}{\alpha_{r}}}
$$

Equation A7.6 is valid when all wind speeds used in Eqs. A7.2 to A7.6 are mean hourly values. If this is not the case, Eq. A7.6 should be modified accordingly. For example, consider a case in which the 3-s wind speed at $10 \mathrm{~m}$ elevation above the ground in the open terrain exposure, $V_{C}^{3-s, 10 \mathrm{~m}}$, is to be rescaled to the mean hourly wind speed at elevation $H$ in the open terrain exposure, $V_{C}^{H}$. Then, the wind speed being sought is calculated as:

$$
V_{C}^{H}=\frac{1}{1.52} V_{C}^{3-s, 10 \mathrm{~m}}\left(\frac{Z_{g, C}}{10}\right)^{\frac{1}{\alpha_{C}}}\left(\frac{H}{Z_{g, r}}\right)^{\frac{1}{\alpha_{r}}}
$$

where $1 / 1.52$ is the ratio of 1-hour averaged speed to 3-s averaged speed at $10 \mathrm{~m}$ elevation above ground in open terrain exposure (see Figure c26.5-1 of ASCE 7-16), and $1 / \alpha_{r}$ is the power law exponent for mean hourly wind speeds in terrain exposure $r(=\mathrm{A}, \mathrm{B}, \mathrm{C}$ or $\mathrm{D})$. Equation A7.7a can be generalized if $V_{C}^{3-s, 10 \mathrm{~m}}$ is transformed to the mean hourly wind speed at elevation $H$ in terrain exposure $r, V_{r}^{H}$

$$
V_{r}^{1 \mathrm{hr}, H}=\gamma V_{C}^{3-\mathrm{s}, 10 \mathrm{~m}}\left(\frac{Z_{g, C}}{10}\right)^{\frac{1}{\alpha_{C}}}\left(\frac{H}{Z_{g, \tau}}\right)^{\frac{1}{\alpha_{3-s, r}}}
$$

where $1 / \alpha_{3-s, r}$ is the power law exponent for 3-s wind speeds in terrain exposure $r$.

The ASCE 7-16 provides the mean hourly wind speed at height $\bar{Z}$ determined from Eq. A7.8, which is equivalent to Eq. A7.7a.

$$
\bar{V}_{\overline{\mathrm{z}}}=V \bar{b}\left(\frac{\bar{Z}}{10}\right)^{\frac{1}{\alpha}}
$$

where $\bar{b}$ and $\alpha$ is the constant listed in Table A1, and $V$ is the basic wind speed averaged in 3 s. Eqs. A7.7b and A7.8 are identical. 
Table A7.1. Terrain exposure constants and power law exponents

\begin{tabular}{ccccc}
\hline Terrain exposure $^{*}$ & $\mathrm{~A}^{* *}$ & $\mathrm{~B}$ & $\mathrm{C}$ & $\mathrm{D}$ \\
\hline $\bar{b}$ & 0.23 & 0.45 & 0.65 & 0.80 \\
$\alpha$ & 2.5 & 4.0 & 6.5 & 9.0 \\
$z_{g}$ & $520 \mathrm{~m}$ & $365.76 \mathrm{~m}$ & $274.32 \mathrm{~m}$ & $213.36 \mathrm{~m}$ \\
\hline
\end{tabular}

* Terrain exposure categories: A = Urban, B = Suburban, C = Open, and D = Water surface.

** Values for the terrain exposure A are from NBC of Canada.

Finally, the variable Ratio_Vs can be calculated and constructed by using Eq. A7.7b as

$$
\begin{aligned}
\text { Ratio_Vs }= & {\left[\begin{array}{lll}
\frac{V_{A}^{1 \mathrm{hr}, H}}{V_{C}^{\text {Avg-time, } 10 \mathrm{~m}}} \frac{V_{B}^{1 \mathrm{hr}, H}}{V_{C}^{\text {Avg-time }, 10 \mathrm{~m}}} \frac{V_{C}^{1 \mathrm{hr}, H}}{V_{C}^{\text {Avg-time, } 10 \mathrm{~m}}} \frac{V_{D}^{1 \mathrm{hr}, H}}{V_{C}^{\text {Avg-time }, 10 \mathrm{~m}}}
\end{array}\right] } \\
& {\left[\begin{array}{l}
\gamma\left(\frac{Z_{g, C}}{10}\right)^{\frac{1}{\alpha_{C}}}\left(\frac{H}{Z_{g, A}}\right)^{\frac{1}{\alpha_{A}}} \\
\gamma\left(\frac{Z_{g, C}}{10}\right)^{\frac{1}{\alpha_{C}}}\left(\frac{H}{Z_{g, B}}\right)^{\frac{1}{\alpha_{B}}} \\
\gamma\left(\frac{z_{g, C}}{10}\right)^{\frac{1}{\alpha_{C}}}\left(\frac{H}{Z_{g, C}}\right)^{\frac{1}{\alpha_{C}}} \\
\gamma\left(\frac{Z_{g, C}}{10}\right)^{\frac{1}{\alpha_{C}}}\left(\frac{H}{Z_{g, D}}\right)^{\frac{1}{\alpha_{D}}}
\end{array}\right] }
\end{aligned}
$$

, or by ASCE 7-16 standard using Eq. A7.8 as

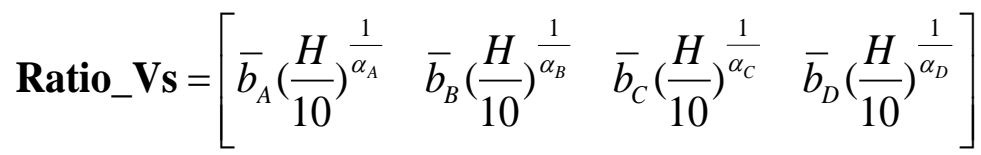

Note that the wind speeds used in the DAD_ESWL software are in the SI unit $(\mathrm{m} / \mathrm{s})$. 


\section{A7.2. Example problems}

Problem 1. Determine the variable Ratio_Vs when the NIST hurricane data are applied to designing a $182.88 \mathrm{~m}$ height building surrounding the suburban and open terrain exposures. The NIST hurricane wind speeds can be accessed via www.nist.gov/wind, and are 1-min averaged in knots at $10 \mathrm{~m}$ above in the open terrain near the coastline.

\section{Solution)}

Due to the wind speeds are in knots, the converting constant can be

$0.447\left(\frac{\mathrm{m} / \mathrm{s}}{\mathrm{mph}}\right) \times 1.15\left(\frac{\mathrm{mph}}{\mathrm{kn}}\right)=0.514$

And the averaging time ratio of 1-hour to 1-min is

$\gamma=\frac{1}{1.24}$

From Table A7.1, the gradient heights $z_{g, A}, z_{g, B}, z_{g, C}$, and $z_{g, D}$ are $520 \mathrm{~m}, 365.76 \mathrm{~m}$, $274.32 \mathrm{~m}, 213.36 \mathrm{~m}$, respectively, and the power law exponents $1 / \alpha_{A}, 1 / \alpha_{B}, 1 / \alpha_{C}$, and $1 / \alpha_{D}$ are $1 / 2.5,1 / 4.0,1 / 6.5$, and $1 / 9.0$, respectively. Thus, the variable Ratio_Vs can be constructed as

$$
\begin{aligned}
& \text { Ratio_Vs }=\left[\begin{array}{llll}
\frac{V_{A}^{1 \mathrm{hr}, H}}{V_{C}^{1 \mathrm{~min}, 10 \mathrm{~m}}} & \frac{V_{B}^{1 \mathrm{hr}, H}}{V_{C}^{1 \mathrm{~min}, 10 \mathrm{~m}}} & \frac{V_{C}^{1 \mathrm{hr}, H}}{V_{C}^{1 \mathrm{~min}, 10 \mathrm{~m}}} & \frac{V_{D}^{1 \mathrm{hr}, H}}{V_{C}^{1 \mathrm{~min}, 10 \mathrm{~m}}}
\end{array}\right] \\
& =\left[\begin{array}{l}
0.514\left(\frac{1}{1.24}\right)\left(\frac{274.32}{10}\right)^{\frac{1}{6.5}}\left(\frac{182.88}{520}\right)^{\frac{1}{2.5}} \\
0.514\left(\frac{1}{1.24}\right)\left(\frac{274.32}{10}\right)^{\frac{1}{6.5}}\left(\frac{182.88}{365.76}\right)^{\frac{1}{4}} \\
0.514\left(\frac{1}{1.24}\right)\left(\frac{274.32}{10}\right)^{\frac{1}{6.5}}\left(\frac{182.88}{274.32}\right)^{\frac{1}{6.5}} \\
0.514\left(\frac{1}{1.24}\right)\left(\frac{274.32}{10}\right)^{\frac{1}{6.5}}\left(\frac{182.88}{213.36}\right)^{\frac{1}{9}}
\end{array}\right]^{\frac{1}{0}} \\
& =\left[\begin{array}{llll}
0.454 & 0.580 & 0.648 & 0.678
\end{array}\right]
\end{aligned}
$$


Problem 2. Determine the variable Ratio_Vs when the synoptic data generated based on 3 -s averaged wind speed in $\mathrm{mph}$ at $10 \mathrm{~m}$ above ground in the open terrain exposure are applied to designing a $160 \mathrm{~m}$ height building.

\section{Solution)}

Due to the wind speeds are in mph, the converting constant can be

$0.447\left(\frac{\mathrm{m} / \mathrm{s}}{\mathrm{mph}}\right)=0.447$

And the averaging time ratio of 1-hour to 3-s is

$$
\gamma=\frac{1}{1.52}
$$

From Table A7.1, the gradient heights $z_{g, A}, z_{g, B}, z_{g, C}$, and $z_{g, D}$ are $520 \mathrm{~m}, 365.76 \mathrm{~m}$, $274.32 \mathrm{~m}, 213.36 \mathrm{~m}$, respectively, and the power law exponents $\alpha_{A}, \alpha_{B}, \alpha_{C}$, and $\alpha_{D}$ are 2.5, 4.0, 6.5, and 9.0, respectively. Thus, the variable Ratio_Vs can be constructed as

$$
\begin{aligned}
& \text { Ratio_Vs }=\left[\begin{array}{llll}
\frac{V_{A}^{1 \mathrm{hr}, H}}{V_{C}^{3 \mathrm{~s}, 10 \mathrm{~m}}} & \frac{V_{B}^{1 \mathrm{hr}, H}}{V_{C}^{3 \mathrm{~s}, 10 \mathrm{~m}}} & \frac{V_{C}^{1 \mathrm{hr}, H}}{V_{C}^{3 \mathrm{~s}, 10 \mathrm{~m}}} & \frac{V_{D}^{1 \mathrm{hr}, H}}{V_{C}^{3 \mathrm{~s}, 10 \mathrm{~m}}}
\end{array}\right]
\end{aligned}
$$

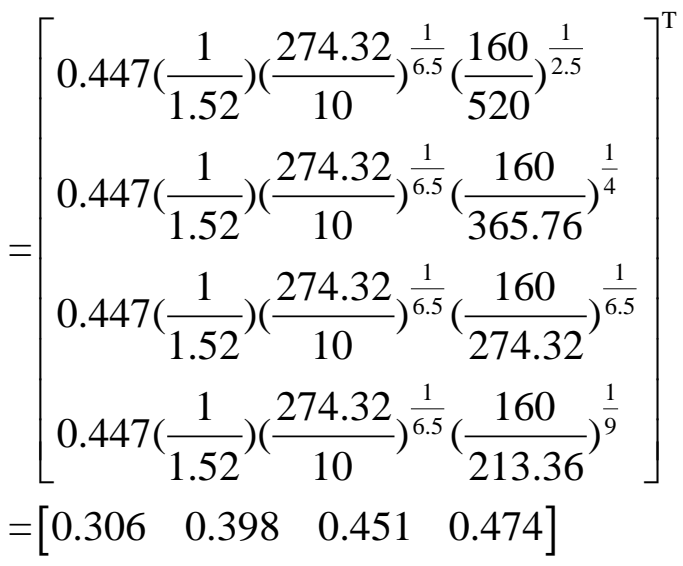

An alternative approach can be developed, wherein the logarithmic law, rather than the power law is used. 